\title{
ON THE COMPLEXITY OF FAILURE A FUNCTIONAL APPROACH TO VIBRATION ANALYSIS
}

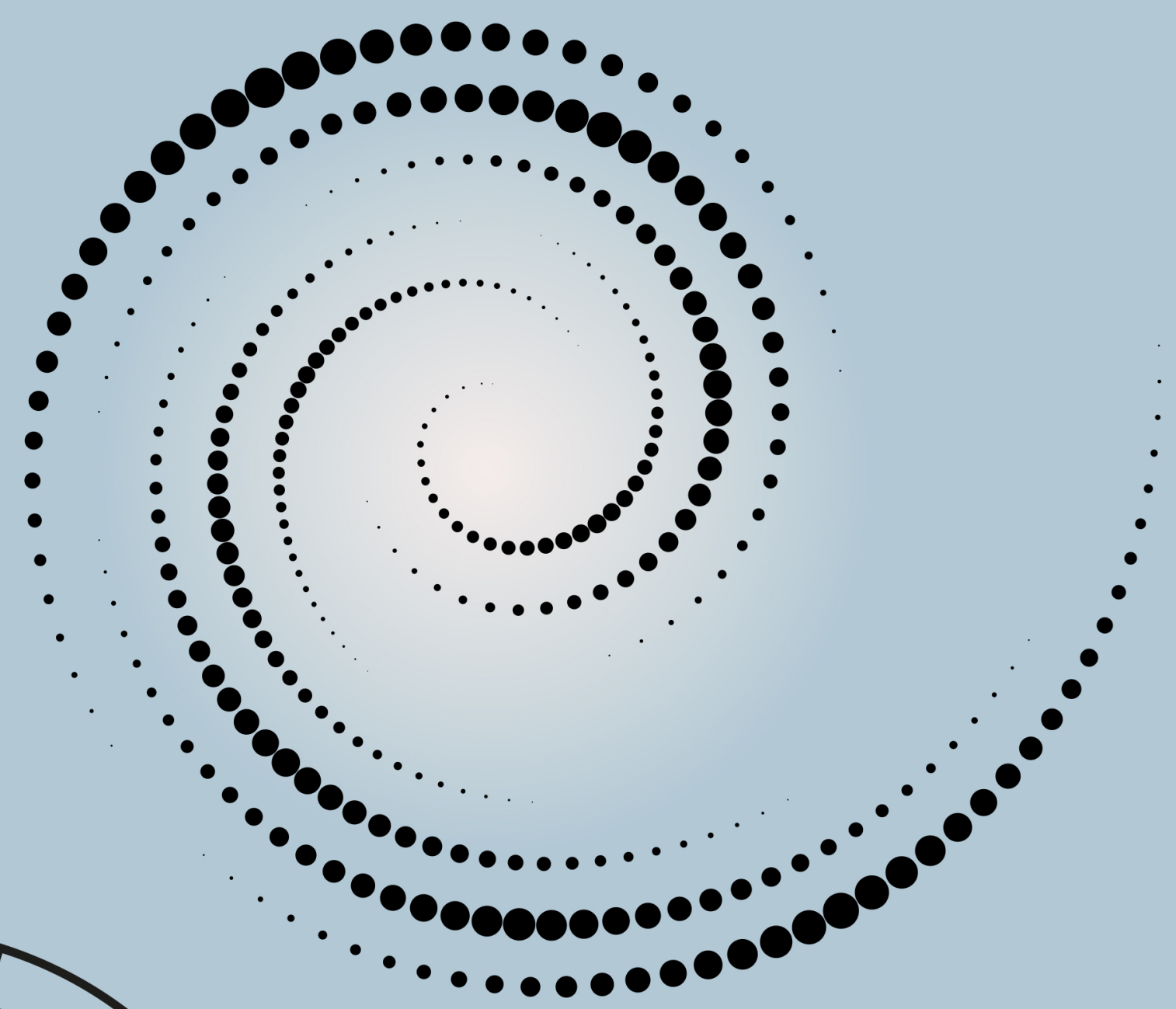

ANDREA SÁNCHEZ RAMÍREZ 


\section{ON THE COMPLEXITY OF FAILURE \\ A FUNCTIONAL APPROACH TO VIBRATION ANALYSIS}

Andrea Sánchez Ramírez 


\title{
ON THE COMPLEXITY OF FAILURE A FUNCTIONAL APPROACH TO VIBRATION ANALYSIS
}

\author{
DISSERTATION
}

to obtain

the degree of doctor at the University of Twente, on the authority of the rector magnificus, prof.dr. T.T.M. Palstra, on account of the decision of the Doctorate Board to be publicly defended on Thursday the $25^{\text {th }}$ of June 2020 at 12:45 hours

by

Andrea Sánchez Ramírez

Born on the 30 ${ }^{\text {th }}$ of April 1982

in Bogota, Colombia 
This dissertation has been approved by:

Supervisor:

Prof.dr.ir. L.A.M. van Dongen

Co-supervisor:

Dr.ir. R. Loendersloot

Cover design: Ilse Modder

Printed by: Gilderprint

ISBN: 978-90-365-5024-6

DOI: $10.3990 / 1.9789036550246$

(C)2020 Enschede, The Netherlands. All rights reserved. No parts of this thesis may be reproduced, stored in a retrieval system or transmitted in any form or by any means without permission of the author. Alle rechten voorbehouden. Niets uit deze uitgave mag worden vermenigvuldigd, in enige vorm of op enige wijze, zonder voorafgaande schriftelijke toestemming van de auteur. 


\section{GRADUATION COMMITTEE:}

Chairman/secretary

Supervisor

Co-supervisor

Members
Prof.dr.ir. H.F.J.M. Koopman

Prof.dr.ir. L.A.M. van Dongen

Dr.ir. R. Loendersloot

Prof.dr.ir. M.B. de Rooij

Prof.dr.ir. J.I.M. Halman

Prof.dr. A. Starr

Prof.dr. S.J. Watson

Dr. K. Dykes

Dr.ir. D. Lutters 

'Life is not what one lived, but what one remembers it and how one remembers it in order to recount it'.

Gabriel García Marquéz 



\section{Preface}

The story of how this thesis came to exist is a nonlinear one.

I developed my interest for the topic of mechanical vibrations early on my bachelor studies on Mechanical Engineering in Colombia. Maybe what make the topic so interesting was the idea that machines could talk, we just needed to learn to listen to them. During my my professional life I have experienced first-hand the potential of vibration analysis for understanding what the machines are telling.

In 2012 I was invited to the project WiBRATE for the integration of IoT technologies and vibration monitoring. This was a fascinating opportunity to unleash the potential of vibration analysis for smart, self-diagnostic systems. And despite the many interesting outcomes, we could not use these technologies beyond localised damage detection. It became clear to me that for the development of smarter, leaner and more robust mechanical systems, we needed to understand better how system failures develop so that we could interpret the associated changes in the vibration response.

This thesis presents the best answer to that question I could find. I wish the reader can find this book as interesting to read as it was for me to write.

Andrea Sánchez Ramírez

A.k.a. 'The Machine Whisperer'

Frederiksberg, Denmark, June 2020. 


\section{Summary}

This dissertation addresses the phenomenon of complex failure in the context of rotating mechanical systems. Specifically, complex failure refers to the irreversible alterations of the load distribution, leading to a heterogeneous degradation of system components. In general complex failures cannot be attributed to a single component damage nor the operational environment alone. Rather these emerge as a combination of factors that undermine system functionality altogether. Complex failures stretch the modelling possibilities from a physics and an empirical perspective to a system-wide scale, limiting the accuracy of their assessment.

This dissertation offers a framework for the study of complex failures. This framework elaborates from the theory of material degradation and extends to the changes in load distribution among system components. Such changes are understood to obey to fundamentally different principles than those used in the design. The framework is guided according to four main elements. The mechanisms and dynamics of complex failures and the methods and instruments necessary to the assessment of the corresponding failure behaviour.

Firstly, the relation between the material degradation and the functional degradation is identified as the main mechanism responsible of the changes in load distribution. Such relation depends on the the dependency of performance and reliability beyond the design range. The increased dependencies facilitate further deviation from the designed load distributions.

Secondly, the dynamics that govern the evolution of failure are guided by new 'organizing' principles, different from the design principles. This thesis describes failure as a transformation that enforces divergent and progressive changes on the system behaviour. This is formulated as three distinct principles of the failure behaviour: the loss energy principle, the kinetic energy principle and the mode-state dependency principle. 
The divergence from the designed behaviour feeds from the change in the energy balance of the system. On the one hand, the available kinetic energy for the system main function is reduced; and on the other hand, there is an increase in the disruption of the potential energy at material and (sub) system level. This is described as the loss energy principle, which manifests itself as system under-performance and increasing system degradation.

The progressive character refers to the gradual deterioration of system functionality through distinct failure states. A failure state denotes an emerging re-organisation of the system load distribution, leading to a heterogeneous deterioration of the components. The variations of the system behaviour for a given failure state are attributed to the kinetic energy content involved. This is modulated by three factors: the functional role of the compromised components in the power transformation, the characteristics of the damage itself, and, the sensitivity to the changing operational environment. This is formulated as the kinetic energy principle.

The progressive character refers to the gradual deterioration of system functionality through distinct failure states. A failure state denotes an emerging re-organisation of the system load distribution, leading to an accelerated deterioration of the components. The variations of the system behaviour for a given failure state are attributed to the kinetic energy content involved. This is modulated by three factors: the functional role of the compromised components in the power transformation, the characteristics of the damage itself, and, the sensitivity to the changing operational environment. This is formulated as the kinetic energy principle.

The transition between failure states, or failure proliferation, is analysed from the functional dependencies between the failures. Such dependency emphasises the synergistic effects of multiple, interacting failure modes that can lead to a higher level degradation than when occurring individually. This is formulated as the modestate dependency principle.

Thirdly, the methods for the observation of complex failure. The mechanism and dynamics form a conceptual basis to approach the failure phenomenon at a system level. This conceptual basis is complemented by a functional approach to vibration analysis, as a comprehensive method to observe the changes in the system behaviour. This approach recognises lateral vibrations as a direct approximation of the destabilisation forces disrupting the system load distribution. The interpretation of the vibration response is therefore evaluated with respect to the system functionality. Thus, the vibration signal is interpreted as a deviation from the designed, linear dynamic response corresponding to the expected functionality. The emerging vibration behaviour is evaluated under an entropic perspective derived from the loss energy principle. This encloses the distinction between the operational and damage- 
induced nature of the forces and resonances responsible of the vibration response, and the characterisation of the interactions between these forces.

The principles of failure behaviour and the functional approach to vibration monitoring are illustrated in two mechanical sub-systems: roller bearings and rotor blade systems. For the bearing case, the vibration behaviour is analysed to characterise the functional effects and load distribution changes associated to component damage. This is carried out through a comparative study of three different systems displaying bearing damage, a lab set-up, a wind turbine generator and a train axle box . These systems enable the comparison of different types of damage and operational environment. For the rotor blade case, the vibration behaviour is analysed for different types of nonlinearities arising from failure, both affecting the structure itself and its input loads. This example is developed through a demonstrator of a bladelike structure representing the operational excitations and boundary conditions of a rotating blade.

Finally, the thesis discusses the instruments for the observation of failure. Specifically, the design requirements for vibration monitoring systems in accordance with the monitoring needs for complex failure are discussed. A systems perspective that integrates the monitored and monitoring system is proposed. 


\section{Samenvatting}

Dit proefschrift behandelt het fenomeen van complexe storingen in roterende mechanische machines. In het bijzonder verwijzen complexe storingen naar onomkeerbare veranderingen in de verdeling van de machinebelasting, wat leidt tot een heterogene degradatie van systeemcomponenten. In het algemeen kunnen complexe storingsmechanismen noch worden toegeschreven aan een enkele beschadigde component, noch alleen aan de operationele omgeving. Deze komen veeleer naar voren als een combinatie van factoren die de gehele systeemfunctionaliteit ondermijnen. Het diagnosticeren en voorspellen van systeemgedrag dat samenhangt met complexe storingsmechanismen is een uitdaging omdat complexe storingsmechanismen de modelleringsmogelijkheden vanuit een fysisch en empirisch perspectief oprekken tot op de schaal van het systeem als geheel.

Dit proefschrift biedt een kader voor de studie van complexe storingsmechanismen. Dit kader gaat uit van de theorie van materiaaldegradatie en strekt zich uit tot de veranderingen in de belastingverdeling tussen systeemcomponenten. Deze veranderingen worden geacht fundamenteel andere principes te volgen dan de in het ontwerp gebruikte principes. Het kader wordt aan de hand van vier hoofdelementen bepaald.

Ten eerste is het/de mechanisme(n) van complex storingsgedrag. De afhankelijkheid tussen de functionele parameters met betrekking tot prestaties en betrouwbaarheid buiten de op lineair gedrag gebaseerde ontwerp principes wordt gedentificeerd als het belangrijkste mechanisme dat verantwoordelijk is voor veranderingen in de belastingverdeling. Dergelijke afhankelijkheden vergroten de complexiteit van het systeem voorbij de ontworpen structurele en functionele relaties.

Ten tweede, de dynamiek die de evolutie van de storing beheerst. Storingsgenduceerde veranderingen van het systeemgedrag worden gedreven door nieuwe 'organiserende' principes, die afwijken van de ontwerpprincipes. Deze dissertatie 
beschrijft storing als een transformatie die uiteenlopende en progressieve veranderingen in het systeemgedrag veroorzaakt. Drie verschillende principes van het faalgedrag worden geformuleerd: het loss energy principle, het kinetic energy principle en het mode-state dependency principle.

De afwijking van het gedrag zoals volgt uit het ontwerp ontstaat door de verandering in de energiebalans van het systeem. Enerzijds wordt de beschikbare kinetische energie voor de hoofdfunctie van het systeem gereduceerd; anderzijds is er een toename van de verstoring van de potentile energie op materiaal en (sub)systeemniveau, die de capaciteit van het materiaal en de structurele configuratie benvloedt. Dit wordt beschreven als het loss energy principle, dat zich manifesteert als een prestatie beneden het nominale niveau en een toenemende degradatie van het systeem.

Het progressieve karakter verwijst naar de geleidelijke verslechtering van de functionaliteit van het systeem door middel van duidelijke storingsomstandigheid. Een storingsincident leidt tot het ontstaan van een herindeling van de systeembelastingverdeling, die leidt tot een meer heterogene slijtage van de componenten. De variaties in het systeemgedrag voor een bepaald storingsincident worden toegeschreven aan de kinetische energie-inhoud. Dit wordt gemoduleerd aan de hand van drie factoren: de functionele rol van de gecompromitteerde componenten in de vermogenstransformatie, de kenmerken van de schade zelf en de gevoeligheid voor de veranderingen in de operationele omgeving. Dit is geformuleerd als het kinetic energy principle.

De overgang tussen storingsincidenten, oftewel de proliferatie van schade en slijtage, wordt geanalyseerd vanuit de functionele afhankelijkheden tussen de ontstane schades. Deze afhankelijkheid benadrukt de synergetische effecten van meervoudige, op elkaar inwerkende storingswijzen die kunnen leiden tot een snellere degradatie dan wanneer deze zich individueel voordoen. Dit is aangeduid als het mode-state dependency principle.

Ten derde, de methoden voor de observatie van complex storingsgedrag. Het mechanisme en de dynamiek vormen een conceptuele basis om storingsfenomenen op systeemniveau te benaderen. Deze conceptuele basis wordt aangevuld met een functionele benadering van de trillingsanalyse, als een uitgebreide methode om de veranderingen in het systeemgedrag te observeren. Deze insteek geeft bijvoorbeeld aan dat de laterale trillingen in een lager beschouwd kunnen worden als een directe benadering van de destabilisatiekrachten die de belastingverdeling van het systeem verstoren. De interpretatie van de trillingsrespons wordt daarom gevalueerd ten opzichte van de functionaliteit van het systeem. Op deze manier wordt het trilsignaal genterpreteerd als een afwijking van de lineaire dynamische respons, zoals uit het ontwerp volgt en overeenkomt met de verwachte functionaliteit. Het ontstane 
trillingsgedrag wordt gevalueerd onder een entropisch perspectief dat is afgeleid van het loss energy principle. Dit betekent dat onderscheid wordt gemaakt tussen de operationele en de door schade genduceerde aard van de krachten en resonanties die verantwoordelijk zijn voor de trillingsrespons en dat de interacties tussen deze krachten gekarakteriseerd worden.

De principes van faalgedrag en de functionele benadering van monitoring op basis van trillingen worden gellustreerd in twee mechanische subsystemen: rollagers en rotorbladsystemen. In het geval van de lagers wordt het trillingsgedrag geanalyseerd om de functionele effecten en de veranderingen in de belastingsverdeling in verband met schade aan componenten te karakteriseren. Dit wordt uitgevoerd door middel van een vergelijkende studie van drie verschillende systemen met lagerschade, een laboratoriumopstelling, een windturbinegenerator en een treinaspot. Deze systemen maken het mogelijk om verschillende soorten schade en de operationele omgeving met elkaar te vergelijken. In het geval van de rotorbladen wordt het trillingsgedrag geanalyseerd aan de hand van verschillende types van niet-lineaire schade als gevolg van schades, die zowel de constructie zelf als de ingangsbelastingen benvloeden. Dit voorbeeld is ontwikkeld met behulp van een demonstrator van een rotorbladachtige structuur die de operationele excitaties en de randvoorwaarden van een roterend blad weergeeft.

Tot slot bespreekt het proefschrift de instrumenten voor de waarneming van storingsgedrag. Specifiek worden de ontwerpeisen voor trillingsmonitoringsystemen in overeenstemming met de monitoringsbehoeften voor complexe storingen besproken. Er wordt een systeemperspectief voorgesteld dat het gemonitorde en monitoringsysteem samenvoegt. 


\section{Contents}

Preface $\quad$ i

Summary $\quad$ iii

Samenvatting vii

Table of Contents $\quad$ Xv

List of Figures $\quad$ xx

List of Tables $\quad$ xxi

Nomenclature $\quad$ xxiii

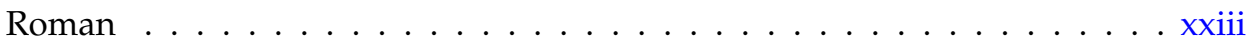

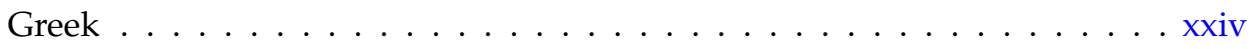

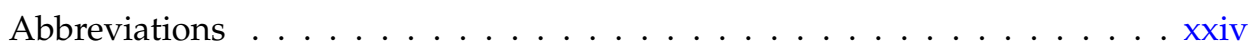

1 Introduction 1

1.1 Complexity of rotating mechanical systems . . . . . . . . . . . . . 1

1.2 System degradation . . . . . . . . . . . . . . . . . . . . 4

1.2.1 Designed, expected ageing ............... 4

1.2.2 Abnormal, unexpected degradation ............ 6

1.3 Vibration analysis . . . . . . . . . . . . . . . . . 9

1.3.1 Early damage detection . . . . . . . . . . . . . . . . . . . . . . . . . . . . 10

1.3.2 Failure proliferation assessment . . . . . . . . . . . 12 
1.4 Thesis goal . . . . . . . . . . . . . . . . . . . . . . 15

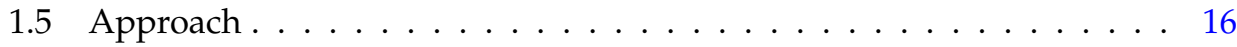

1.5.1 Designed complexity . . . . . . . . . . . . . . . . . 16

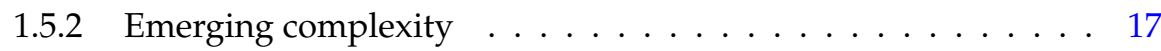

1.6 Contributions . . . . . . . . . . . . . . . . . . . . . 19

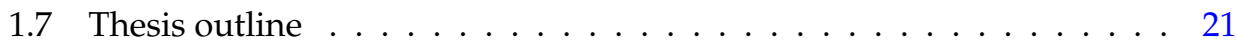

I On Failure Complexity 23

2 Mechanism of complex failure 25

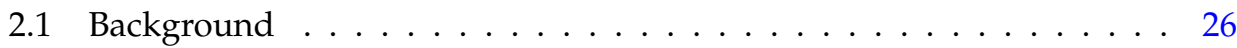

2.1.1 Failure mechanisms . . . . . . . . . . . . . . . . . 27

2.1.2 Intra-component dependencies . . . . . . . . . . . . . 28

2.1.3 Inter-component dependencies . . . . . . . . . . . . . . . 31

2.2 Performance and reliability dependencies . . . . . . . . . . 33

2.2.1 Designed dependency . . . . . . . . . . . . . . 34

2.2.2 Failure-induced dependencies . . . . . . . . . . . . . . . 34

2.3 Rotating mechanical systems functionality . . . . . . . . . . 35

2.3.1 Performance . . . . . . . . . . . . . . . . . . . . . . . . . 39

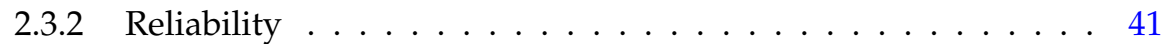

2.4 Failure states . . . . . . . . . . . . . . . . . . . . 43

2.4.1 Effect on performance and reliability . . . . . . . . . . . 44

2.4.2 Composition of failure transformation . . . . . . . . . . . . 44

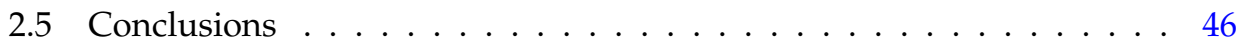

3 Dynamics of complex failure 49

3.1 Failure behaviour . . . . . . . . . . . . . . . . . 50

3.2 Divergence . . . . . . . . . . . . . . . . . . 51

3.3 Progressive: within-state variations . . . . . . . . . . . . . . . 52

3.4 Progressive: between-state variations $\ldots \ldots \ldots \ldots \ldots \ldots$

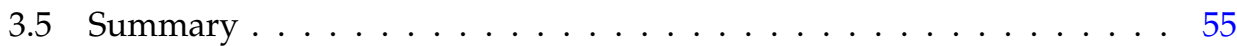




\section{A Functional Approach to Vibration Analysis}

4 Entropy: Vibration response analysis $\quad 59$

4.1 Introduction to the second part $\ldots \ldots \ldots \ldots \ldots$

4.2 Vibration as loss energy . . . . . . . . . . . . . . . . . 60

4.3 Elements of vibration response $\ldots \ldots \ldots \ldots \ldots \ldots \ldots$

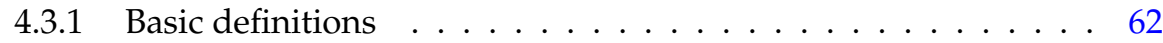

4.3.2 Signal decomposition . . . . . . . . . . . . . . . 64

4.4 Non-linear interactions . . . . . . . . . . . . . . . . . . 66

4.4 .1 Harmonic distortion . . . . . . . . . . . . . . . . . 67

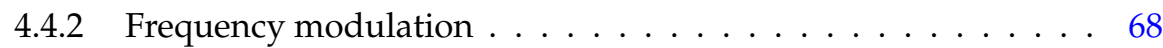

4.4 .3 Amplitude modulation . . . . . . . . . . . . . . . . . . 69

4.4 .4 Resonance state . . . . . . . . . . . . . . . . . . . . . . 70

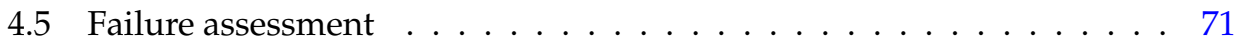

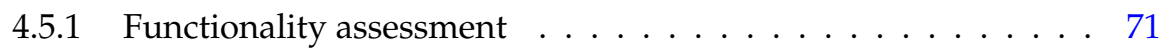

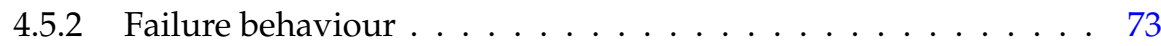

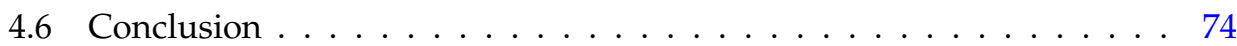

5 Uncertainty: Roller bearings case 75

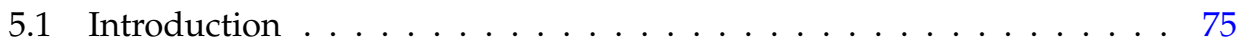

5.1.1 Uncertainty principle and vibration behaviour . . . . . . . 75

5.1 .2 Roller bearing diagnostics . . . . . . . . . . . . . . . 77

5.1 .3 Chapter structure . . . . . . . . . . . . . . . . 78

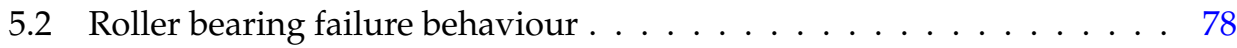

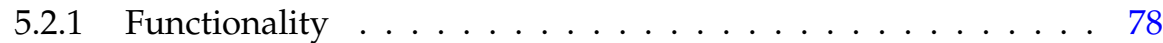

5.2 .2 Bearing damage . . . . . . . . . . . . . . . . . 79

5.2 .3 Vibration behaviour . . . . . . . . . . . . . 81

5.3 Case I: Lab set-up . . . . . . . . . . . . . . . . . . . . . 85

5.3.1 Signal characterisation . . . . . . . . . . . . . . . 86

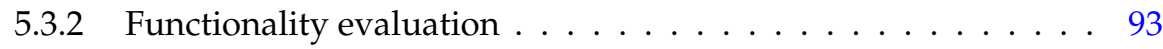

5.3.3 Load distribution evaluation . . . . . . . . . . . . . . . . . 94

5.4 Case II: Wind turbine generator . . . . . . . . . . . . . . . . . . 95

5.4 .1 Signal characterisation . . . . . . . . . . . . . . . . . 97 
5.4 .2 Functionality evaluation . . . . . . . . . . . . . . . . 99

5.4 .3 Load distribution evaluation . . . . . . . . . . . . . 100

5.5 Case III: Train axle box . . . . . . . . . . . . . . . . . . . . . . . 103

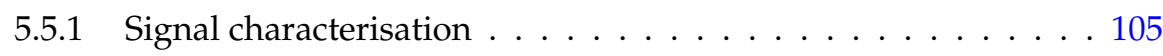

5.5 .2 Functionality evaluation . . . . . . . . . . . . . . 111

5.5.3 Load distribution evaluation . . . . . . . . . . . . . . . 112

5.6 Discussion . . . . . . . . . . . . . . . . . . . . . . . . 113

5.7 Conclusion . . . . . . . . . . . . . . . . . . . . . 117

6 Complexity: Rotor-blade systems case 119

6.1 Rotor blade demonstrator . . . . . . . . . . . . . . . . . . 121

6.1.1 Rotor-blade systems . . . . . . . . . . . . . . 121

6.1 .2 Demonstrator . . . . . . . . . . . . . . . . 123

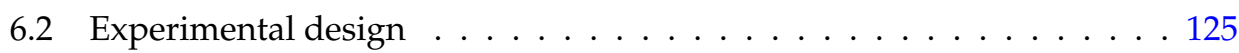

6.2.1 Test variables . . . . . . . . . . . . . . . . . . . . . . . 125

6.2 .2 Procedure . . . . . . . . . . . . . . . . . . . . 127

6.3 Vibration response analysis . . . . . . . . . . . . . . . . . . . . . 129

6.3.1 Response at excitation frequency . . . . . . . . . . . . . . . 129

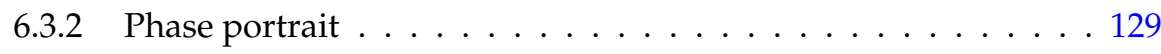

6.3.3 Load distribution analysis . . . . . . . . . . . . . . . . 130

6.4 Results . . . . . . . . . . . . . . . . . . . . . 133

6.4.1 Characterisation of individual modes . . . . . . . . . . . 133

6.4.2 Mode-state dependency analysis . . . . . . . . . . . . . 137

6.5 Discussion . . . . . . . . . . . . . . . . . . . . . . . . . 144

6.6 Conclusion . . . . . . . . . . . . . . . . . . . . . 144

III Design implications and Closing 147

7 Vibration monitoring systems $\quad 149$

7.1 Design framework . . . . . . . . . . . . . . . . . . 150

7.1.1 Monitored system . . . . . . . . . . . . . . . . . 152

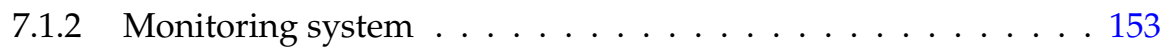


7.2 Stakeholder analysis . . . . . . . . . . . . . . . . . 157

7.2 .1 Monitoring strategy $\ldots \ldots \ldots 158$

7.2.2 Monitoring implementation . . . . . . . . . . . . . . . . . 159

7.3 Conclusions . . . . . . . . . . . . . . . . . . . . 160

8 Conclusions and Recommendations 163

8.1 Understanding the mechanisms of complex failure: the why . . . . 164

8.2 Understanding the dynamics of complex failure: the how . . . . . . . 165

8.3 Interpreting vibration behaviour: the method . . . . . . . . . 166

8.4 Observing complex failure: the instruments . . . . . . . . . . . 167

8.5 Recommendations . . . . . . . . . . . . . . . . . . . . 168

8.5.1 Designing leaner mechanical systems . . . . . . . . . 168

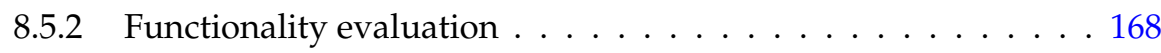

8.5.3 Distributed sensor networks _. . . . . . . . . . . . . . 169

8.6 Final remarks . . . . . . . . . . . . . . . . . . . . . . 169

$\begin{array}{ll}\text { References } & 170\end{array}$

A Roller Bearings Vibration $\quad 181$

A.1 Undamaged behaviour . . . . . . . . . . . . . . . . . . 182

A.2 Quantitative variations . . . . . . . . . . . . . . . . 184

A.3 Qualitative variations . . . . . . . . . . . . . . . . . . . 186

A.3.1 EMD analysis . . . . . . . . . . . . . . . . . . . 191

$\begin{array}{ll}\text { Acknowledgements } & 195\end{array}$

$\begin{array}{ll}\text { About the autor } & 197\end{array}$ 


\section{List of Figures}

1.1 Definition of complexity . . . . . . . . . . . . . 2

1.2 Representation of a system and the factors that influence its lifetime . . 5

1.3 Uncertainty sources related to failure types . . . . . . . . . 7

1.4 Changes in the vibration behaviour under complex failure. . . . . . 10

1.5 Instruments for industrial vibration monitoring. . . . . . . . . . . 11

1.6 Representation of the designed complexity. . . . . . . . . . . . 17

1.7 FBS descriptions in the design and failure contexts. . . . . . . . . . 18

1.8 Representation of the designed complexity. . . . . . . . . . . . . . 19

1.9 System behaviour change due to failure . . . . . . . . . . . . 20

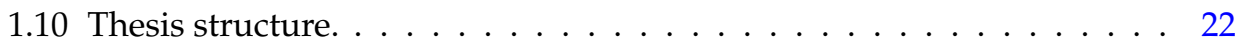

2.1 Dependencies at the material, component and system level. . . . . . 26

2.2 Representation of complexity at component-level . . . . . . . . . 28

2.3 Interaction graphs for intra-component dependencies . . . . . . . . . 29

2.4 Types of failure propagation . . . . . . . . . . . . . . 32

2.5 Stiffness role on component functionality. . . . . . . . . . . . 33

2.6 Performance and reliability dependency. . . . . . . . . . . . 34

2.7 Influence of failure on performance and reliability dependency. . . . . 35

2.8 Complex failure onset and proliferation. . . . . . . . . . . 36

2.9 Designed, system level performance and reliability dependency. . . . . 43

2.10 Example of failure state . . . . . . . . . . . . . . . . 46

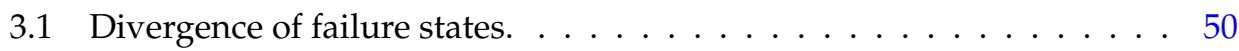


3.2 Failure state - failure mode dependency types. . . . . . . . . . . 53

3.3 Dependency between failure states and incoming failure mode. . . . . 54

4.1 Guiding questions for comprehensive failure assessment. . . . . . . . . 60

4.2 Elements of the vibration phenomenon. . . . . . . . . . . . 62

4.3 Lateral vibration due to residual forces. . . . . . . . . . . . . . . . 63

4.4 Example of Frequency Response Function. . . . . . . . . . . . . . . 65

4.5 Example of Harmonic Distortion. . . . . . . . . . . . . . . . . . . 67

4.6 Example of Frequency Modulation. . . . . . . . . . . . . . . . . 68

4.7 Example of independent vibration responses. . . . . . . . . . . . . . . 69

4.8 Example of Amplitude Modulation. . . . . . . . . . . . . . . . . . . . . 69

4.9 Example of Resonance. . . . . . . . . . . . . . . . . . . . . 70

4.10 Failure progression characterisation by vibration analysis. . . . . . . 73

5.1 Characterisation of a failure state using vibration analysis. . . . . . . 77

5.2 Bearing functions: structural support and free rotation. . . . . . . . 79

5.3 Example of impulse response. . . . . . . . . . . . . . . . . . 81

5.4 Stages of bearing damage $\ldots \ldots \ldots \ldots \ldots \ldots \ldots$

5.5 Lab set-up for artificial damage evaluation . . . . . . . . . . . 85

5.6 Crossing of the ball element passing over the outer race crack. . . . . . 88

5.7 Identification of driving forces at the spectrum. . . . . . . . . . . . 89

5.8 Envelope analysis for identification of driving forces. . . . . . . . . . . 89

5.9 TKEO analysis for signal filtered at $H_{s t} \ldots \ldots \ldots \ldots \ldots$

5.10 TKEO analysis for signal filtered at $H_{d} . \ldots \ldots \ldots \ldots \ldots$

5.11 Feature comparison for the reference and damage cases. . . . . . . . 93

5.12 Load distribution assessment by TKEO exponents. . . . . . . . . . . . 95

5.13 Wind turbine drive train. . . . . . . . . . . . . . . . . 96

5.14 Peak frequencies of TKEO spectra at M10. . . . . . . . . . . . . 99

5.15 Evaluation of the structural support function. . . . . . . . . . . . . 100

5.16 Classification for M6 . . . . . . . . . . . . . . . . . . . . . . . . . . 101

5.17 Classification for M10 . . . . . . . . . . . . . . . . . . . . . . . . 102

5.18 TKEO spectra for the main structural resonances. . . . . . . . . . . 102

5.19 TKEO spectra for the damage-induced resonances. . . . . . . . . . . 103 
5.20 Train axle box. . . . . . . . . . . . . . . . . . . . . . . . . . . 104

5.21 Classification of instantaneous vibration for bearing A at $5 \mathrm{~m} / \mathrm{s} \ldots 107$

5.22 Classification of instantaneous vibration for bearing $C$ at $5 \mathrm{~m} / \mathrm{s} \ldots 108$

5.23 EMD comparison for stable excitation bearing A at $5 \mathrm{~m} / \mathrm{s} \ldots \ldots \ldots$

5.24 EMD comparison for stable excitation bearing $C$ at $5 \mathrm{~m} / \mathrm{s}$. . . . . . . 109

5.25 IMF time waveform and TKEO spectra for bearing $C$ at $5 \mathrm{~m} / \mathrm{s} . \ldots \ldots 110$

5.26 Evaluation support function at stable environment. . . . . . . . . . . 111

5.27 Evaluation support function at random environment. . . . . . . . . . 112

5.28 EMD comparison for stable excitation bearing $C$ at $5 \mathrm{~m} / \mathrm{s} \ldots \ldots \ldots 113$

6.1 Articulated rotor blade systems. . . . . . . . . . . . . . . . . 122

6.2 Demonstrator of rotor blade systems. . . . . . . . . . . . . . . . 124

6.3 Reference condition $H P: a$ ) time waveform, $b$ ) spectrum. . . . . . . 126

6.4 Failure behaviour of demonstrator under failure. . . . . . . . . . . . 128

6.5 Overview of test cases . . . . . . . . . . . . . . . . . . 128

6.6 Phase portraits for variations on amplitude and phase at 3X. . . . . 130

6.7 Mode shapes for test beam. . . . . . . . . . . . . . . . . . . 131

6.8 Harmonic analysis for reference case. . . . . . . . . . . . . . . . 132

6.9 Comparison of normalised $X_{1 \Omega}$ for individual test cases. . . . . . . 133

6.10 Tip response analysis for reference and structural variations cases. . . . 134

6.11 Combined effects of added mass and lower torsional stiffness. . . . . . 135

6.12 Tip response analysis for excitation input variations. . . . . . . . . . 135

6.13 Load distribution for lagging and harmonic distortion. . . . . . . . 136

6.14 Failure states due to individual test cases. . . . . . . . . . . . . 137

6.15 Normalised tip response for various failure states. . . . . . . . . . . 138

6.16 Phase portraits of failure mode dependency . . . . . . . . . . . . . 139

6.17 Mapping of magnitude and phase for $X_{3 \Omega} \ldots \ldots \ldots \ldots \ldots \ldots$

6.18 Comparison of participation factors for varying excitation inputs. . . . 142

6.19 Tip response for medium torsional spring, added mass and lagging. . 143

6.20 Lagging and harmonic distortion for the reference and medium torsional spring. . . . . . . . . . . . . . . . . . . . . 143

7.1 FBS for the monitored and monitoring systems. . . . . . . . . . 150 
7.2 FBS-based requirements for vibration monitoring systems. . . . . . . 151

7.3 FBS for the monitored system . . . . . . . . . . . . . . . . . . . . . . . . . . . . . . . . .

7.4 FBS for the monitoring system . . . . . . . . . . . . . . . . . . . . . . . . . . . . . . . . . . . .

7.5 Centralised and distributed sensor networks. . . . . . . . . . . . . . . . . . . . . . . . . . . . . . .

7.6 Information exchange between stakeholders. . . . . . . . . . . 158

7.7 Definition of monitoring strategies. . . . . . . . . . . . . . . 159

7.8 Definition of monitoring implementation. . . . . . . . . . 160

A.1 Reference case for lab set-up case. . . . . . . . . . . . . . . . . 183

A.2 Classification for M10 . . . . . . . . . . . . . . . . . . 185

A.3 Detail normalised PSD cumulative . . . . . . . . . . . . . 185

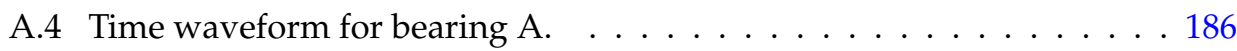

A.5 Frequency distribution for stable and random. . . . . . . . . . . 187

A.6 Frequency distribution for impact and random+impact. . . . . . . . . . . . . . . . . . . . . .

A.7 Classification of instantaneous states . . . . . . . . . . . . . . . 190

A.8 Response analysis for stable state. . . . . . . . . . . . . . . . . . . 191

A.9 Maximum frequency for IMFs versus $r m s$ for bearing A. . . . . . . 193

A.10 Maximum frequency for IMFs versus rms for bearing A. . . . . . . . 194 


\section{List of Tables}

2.1 Classification of failure mechanisms. . . . . . . . . . . . . . 27

4.1 Decomposition of the vibration response from structural dynamics and rotating dynamics. . . . . . . . . . . . . . 64

5.1 Test information for lab set-up case . . . . . . . . . . . . . . 86

5.2 Input signals and spectrograms for lab set-up case. . . . . . . . . . . 87

5.3 Comparison TKEO analysis for reference and damage cases. . . . . . . 91

5.4 Derived frequencies for operational and damage-induced forces. . . . 92

5.5 Bearing information for wind turbine generator case. . . . . . . . . 96

5.6 Input signals and for wind turbine generator case. . . . . . . . . . 97

5.7 Linear regression parameters . . . . . . . . . . . . . . . . 100

5.8 Bearing information for train axle box case. . . . . . . . . . . . . . 104

5.9 Input signals and spectrograms for the train axle box case. . . . . . 106

5.10 Comparison of vibration elements and load distribution for the different bearing damage cases. . . . . . . . . . . . . . . . . . 115

6.1 FBS decomposition for helicopter main rotor blades. . . . . . . . . . 123

6.2 Natural frequencies for the rigid body, first and second elastic flapping modes of the test beam. . . . . . . . . . . . . . . . 131

A.1 Classification parameters for generator bearing signals . . . . . . 184

A.2 Classification parameter for railway bearing signals . . . . . . . 188 


\section{Nomenclature}

\section{Roman}

A rate of degradation for main components [-]

$C$ set of all system components [-]

$C_{\text {main }}$ set of main system components [-]

$C^{\prime} \quad$ subset of components affected by failure [-]

$C \backslash C^{\prime} \quad$ subset of components not affected by failure [-]

$f \quad$ individual function [-]

$f_{p} \quad$ performance requirement $\quad[-]$

$f_{r} \quad$ reliability requirement $[-]$

$F_{o p} \quad$ Operation induced lateral forces $\quad[-]$

$F_{Q} \quad$ Component of $F_{o p}$ contributing to system torque [-]

$F_{d} \quad$ Damage induced lateral forces [-]

$F_{X} \quad$ Component of $F_{o p}$ contributing to lateral vibrations [-]

$H_{s t} \quad$ Structural resonance [-]

$H_{d} \quad$ Damage induced resonance [-]

$m \quad$ material properties

$Q \quad$ System torque [-]

$R_{\text {ageing }} \quad$ normal, expected ageing $\quad[-]$

$R_{\text {long }} \quad$ long-term reliability [-]

$R_{\text {short }}$ short-term reliability

$S_{\text {in }} \quad$ input state [-]

$S_{\text {out }} \quad$ output state [-]

$T \quad$ overall system functionality [-]

$T^{d} \quad$ dynamic aspects of the system [-]

$T_{0} \quad$ designed state [-]

$T_{i} \quad$ failing state [-]

$X \quad$ vibration response $\quad[-]$ 


\section{Greek}

$\delta \quad$ failure mode-state dependency $\quad[-]$

$\Omega \quad$ Fundamental frequency [-]

$\theta \quad$ failure mode [-]

$\theta^{d} \quad$ description of $\theta$ from a dynamic perspective [-]

$\Theta \quad$ failure state [-]

$\Theta^{d} \quad$ description of $\Theta$ from a dynamic perspective [-]

$\chi \quad$ individual constituents of the vibration response [-]

\section{Abbreviations}

$\begin{array}{ll}\text { AM } & \text { Added Mass } \\ \text { BFF } & \text { Bearing Failure Frequency } \\ \text { DSN } & \text { Distributed Sensor Networks } \\ \text { EMD } & \text { Empirical Mode Decomposition } \\ \text { FBS } & \text { Function-Behaviour-Structure } \\ \text { FE } & \text { Flapping Elastic } \\ \text { FFT } & \text { Fast Fourier Transform } \\ \text { FRF } & \text { Frequency Response Function } \\ \text { HD } & \text { Harmonic Distortion } \\ \text { HSA } & \text { Hydraulic Servo Actuator } \\ \text { IoT } & \text { Internet-of-Things } \\ \text { IMF } & \text { Internal Mode Function } \\ \text { ODS } & \text { Operational Deflection Shape } \\ \text { PFM } & \text { Physical Functional Model } \\ \text { RB } & \text { Rigid Body } \\ \text { ROT } & \text { Rotational speed } \\ \text { RUL } & \text { Remaining useful life } \\ \text { TS } & \text { Torsional Spring } \\ \text { TKEO } & \text { Teager-Kaiser Energy Operator } \\ \text { TTF } & \text { Time-to-Failure }\end{array}$




\section{Chapter 1}

\section{Introduction}

Seizing the opportunity to limit the worst effects of climate change requires a swift departure from our current economic, industrial and energy systems. The circular economy and energy transition are crucial to this. Circular economy offers an opportunity to limit resource exploitation, hence to reduce the environmental and societal costs of its extraction. This goal requires extending the operational life of products, equipment and infrastructure, thus improving the productivity of these resources. The green transition requires the quick replacement of the highly intensive $\mathrm{CO} 2$ energy systems with renewable systems. Although renewable's supporting technologies have evolved at an incredible pace, ensuring their reliable operation is still crucial for accelerating their deployment. Improving productivity, efficiency and reliability is therefore, more than ever, a main priority of designers, operators and maintainers.

\subsection{Complexity of rotating mechanical systems}

Rotating mechanical systems form the pillar of our current methods of energy production, transportation and industrial activity. This category refers to the machines that utilise high load-bearing mechanical structures and mechanisms for the power exchange between a fluid or electromagnetic field and a rotor. Wind turbines, helicopters and electric motors are prototypical examples of these systems. The continuous improvement of these machines is an essential goal for achieving a circular and de-carbonised economy in the coming decades [1].

Rotating mechanical machines are complex systems. As systems these entail a large amount of specialised parts interacting through coordinated activities, which together fulfil the expected requirements within accepted tolerance levels. In words 
of Russell L. Ackoff ${ }^{1}$ a system is 'not (just) the sum of its parts, but the product of the interactions of those parts'. Furthermore, rotating mechanical systems must comply with multiple and often conflicting functional requirements. For instance to be economically competitive machines must not only abide by tight constraints, but also achieve minimum energy consumption and sustained functionality over years of operational life. In this sense, their designed complexity is reflected in the difficulty of achieving an overall advantageous set of functions through a strategic configuration that satisfies the most important requirements.

One of the main goals of design is to resolve functional contradictions by making explicit the conflicting interactions between functional requirements within an expected environment. However, making these conflicts explicit is limited by the lack of knowledge about the interactions between functional requirements within an uncertain reality. In other words, it is not possible to fully identify and quantify all conflicting interactions.

\section{COMPLEXITY}

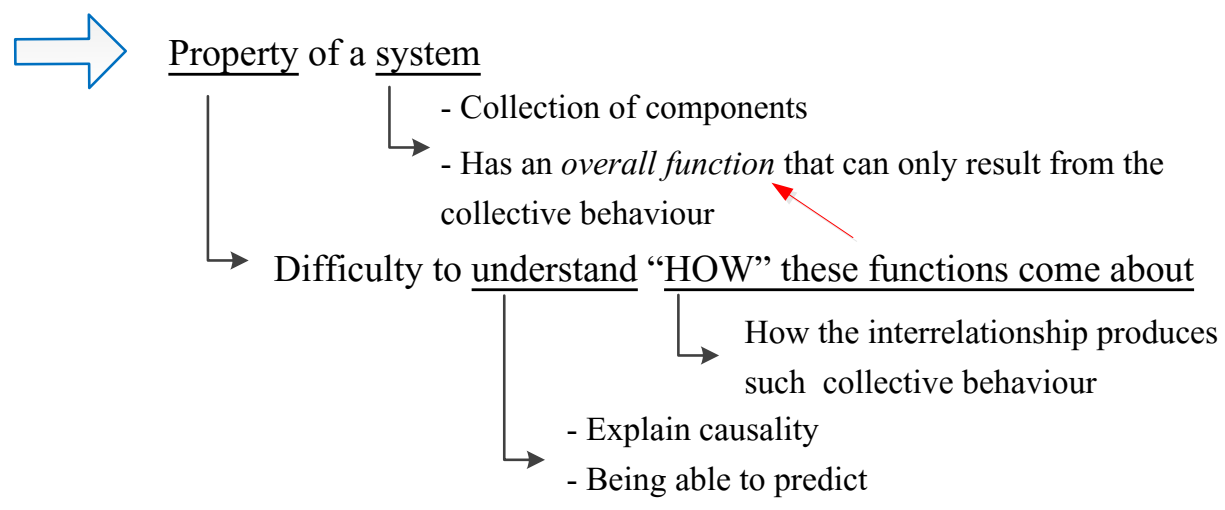

Figure 1.1: Definition of complexity as adopted from Lee [2].

The uncertainty about negative interdependencies makes rotating mechanical machines complex systems. Complexity, as defined by Lee [2], refers to the 'property of a system that makes it difficult to understand as a whole through the collection of knowledge about its constituents'. The meaning of 'understanding' in this context is being able to explain causality and thus being able to predict behaviour (output) given initial conditions (input), as illustrated in Figure 1.1.

\footnotetext{
${ }^{1}$ Russell Lincoln Ackoff (1919 2009) was a pioneer in the field of operations research, systems thinking and management science. Source: Wikipedia
} 
Design lowers the functional complexity by reducing the uncertainty about requirement interdependencies [3]. Such reduction occurs by identifying and quantifying the degree to which functional requirements collide. The final outcome of this part of the design process is to higher structural simplicity [4]. This means that despite the myriad of components specifications necessary for the construction of a system, these specifications are less contingent on (negative) interdependencies.

Among the different types of functional requirements, expectations for component and overall system useful life are highly prone to uncertainty [5]. This is because the extent to which functions interact negatively throughout the entire system lifetime, either due to normal ageing or by the effect of external events, cannot be fully anticipated. In this context failure emerges unexpectedly and unpredictably as a result of the uncertainty regarding system degradation patterns [6]. This is illustrated in the wind turbine gearbox failure in Example 1.

\section{Example 1. Gearbox failure in a wind farm -Part I .}

In 2011 at a wind farm in Oklahoma,USA, the operators at the central control room discovered that a turbine had stopped operating due to a high temperature error. A mild increase on the turbine tower vibrations was observed, yet it was still bellow the warning level. The operator could not restart the turbine remotely after following the procedure for this minor problem, hence a maintenance team had to perform an inspection at the location of the machine. As soon as the technicians opened the tower door, it became clear why the turbine could not start easily. There was oil dripping from the nacelle located at a height of 65 metres. The gearbox had collapsed.

The accident investigation revealed that the cause of the damage was cracking of the bearings at the high speed shaft of the gearbox. According to the manufacturer, the problem occurred because the thermal treatment of the bearings was incorrect. More worrisome yet, there was a high possibility that more turbines had the same faulty components.

The manufacturer offered to exchange all the faulty gearboxes. Yet the main challenge for the wind farm operators and maintenance team was to avoid catastrophic failures. An inspection crew was hired to identify the turbines with symptoms of bearing damage and to prioritise the most severely affected turbines. 
Failure causes the partial or full impairment of system functionality, either from a reliability or performance perspective. The effects of failure on system reliability include inoperative states due to simple errors identified by the surveillance systems, repetitive component exchange or even catastrophic events that compromise the integrity and safety of the systems. Performance effects refer to the lower quality in the system main function. Although performance issues are often less urgent than issues of reliability, these represent significant losses for overall system profitability [7].

Failure can also be understood as a transformation of the designed system. The transformation develops through an onset phase at each component and a proliferation phase where failure achieves system-level effects. On the one hand, component failure is attributed to the imbalance between the component loads and the materialload carrying capacity. On the other hand, failure proliferation is associated with the way that system functions gradually become compromised. This is regulated by the capacity of the unaffected components given the altered load distribution. As the remaining capacity of the unaffected components and the changes in the load distribution are generally unknown, the path from component to system failure is highly uncertain.

A major concern about failure is its difficulty to be understood. According to Lee's definition [2], this is reflected in the inability to explain the factors that trigger failure onset and to predict its proliferation. This difficulty to understand failure hinders the possibility of discerning key lessons from failure occurrences, which could otherwise serve as valuable feedback for improving system design.

In conclusion, while design aims to reduce uncertainty about interdependencies on the functional requirements in order to achieve clear specifications for the construction phase, failure increases the system uncertainty in an opposite direction: from unexpected material degradation through unknown failure proliferation paths that hinder system functionality. Thus, the lack of understanding about the factors that trigger and exacerbate failure increases the uncertainty about a system functionality over its entire useful life. This means that failure increases system complexity.

\subsection{System degradation}

\subsubsection{Designed, expected ageing}

As discussed, uncertainty is a main driver of system complexity. Reducing uncertainty about the factors that influence component and system useful life is key to ensuring system reliable operation. System reliability is dependent on three intermediate variables [8]: 
(a) the load-carrying capacity against plausible failure mechanisms for each of the individual components;

(b) the operational environment throughout the entire useful life; and,

(c) the load distribution over components.

Thus, the designed system lifetime depends on multiple load -carrying capacity relations for the relevant failure mechanism at systems' main components, where the capacity is based on $a$ and the load by $b$ and $c$. This is represented in Figure 1.2

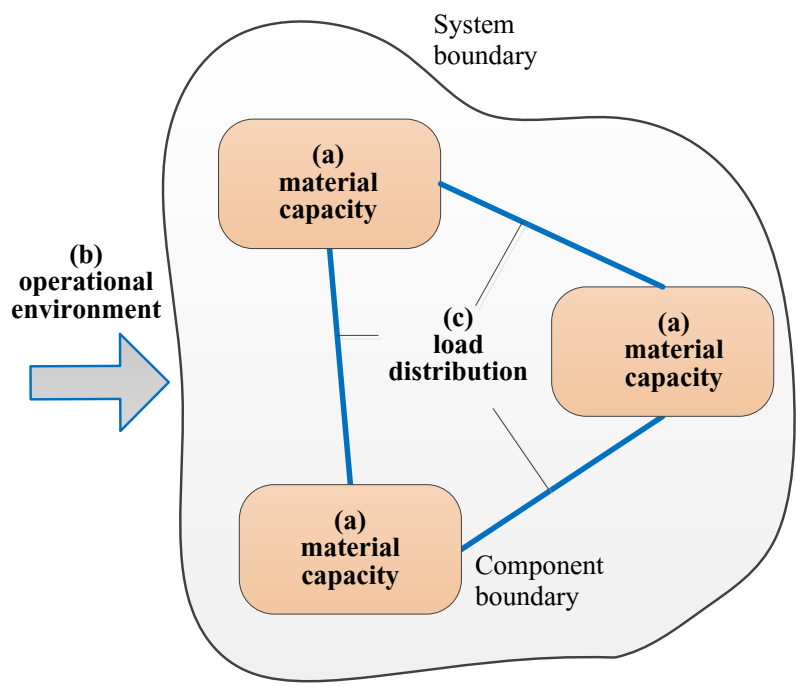

Figure 1.2: Representation of a system and the factors that influence its lifetime: component capacity (a), operational environment (b) and load distribution (c).

Each of the three categories has an implicit uncertainty that must be reduced during design. For instance component reliability tests are used to reduce aleatoric uncertainty through survival reliability tests, and extensive measurement campaigns are carried out to characterise the operational environment [9]. The guarantee for stable load distributions comes through adopting different load scenarios, which include abnormal operational states in addition to the main operation envelope [10].

Despite these measures to prevent failure onset (component failure), systems are required to adopt additional measures to limit the risk of failure proliferation. Safety factors and functional redundancy are measures for avoiding changes in the load 
distribution as a consequence of the failure of adjacent components. This construction leads to degradation of each component (consumption of its capacity) occurring independently from that of other components.

The philosophy of failure independence is not penalty-free, as providing each component with sufficient capacity for independent degradation implies heavier, less efficient and more expensive systems. The advantage of homogeneous material capacity consumption of all components must be balanced against achieving an overall advantageous set of functions. Hence designers are obliged to choose between cost, efficiency and reliability.

\subsubsection{Abnormal, unexpected degradation}

Failure occurrence results as a consequence of the uncertainties in the carryingcapacity and load relations that underlie system reliability. Three types of failure are identified: simple, complicated and complex failure. These types are traced back to lower material capacity, unanticipated operational environment and altered load distribution (Figure 1.3) respectively:

Simple failure develops due to a lower material capacity than expected. This type of failure does not imply permanent changes in the load distribution. Thus, the load distribution is restored after opportune replacement of the faulty component.

Complicated failure occurs due to an unanticipated operational environment. This means that the stress level changes proportionally for all components. Thus, in complicated failures the system as a whole experiences an accelerated degradation, yet without interdependencies between failing components and load redistribution.

Complex failure is attributed to irreversible changes in the system load distribution. In this case the components degrade heterogeneously and disproportionately with respect to the system-level loading. The disrupted component interactions increase the uncertainty on the forthcoming load redistributions, and hence how other components will eventually fail. Additionally, the originally designed load distribution cannot be restored simply by the exchange of the failed components due to the accumulated damage at other affected components.

The wind turbine presented in Example 1 corresponds to a complex failure. The initial damage at the bearing damage propagated throughout the entire gearbox up 


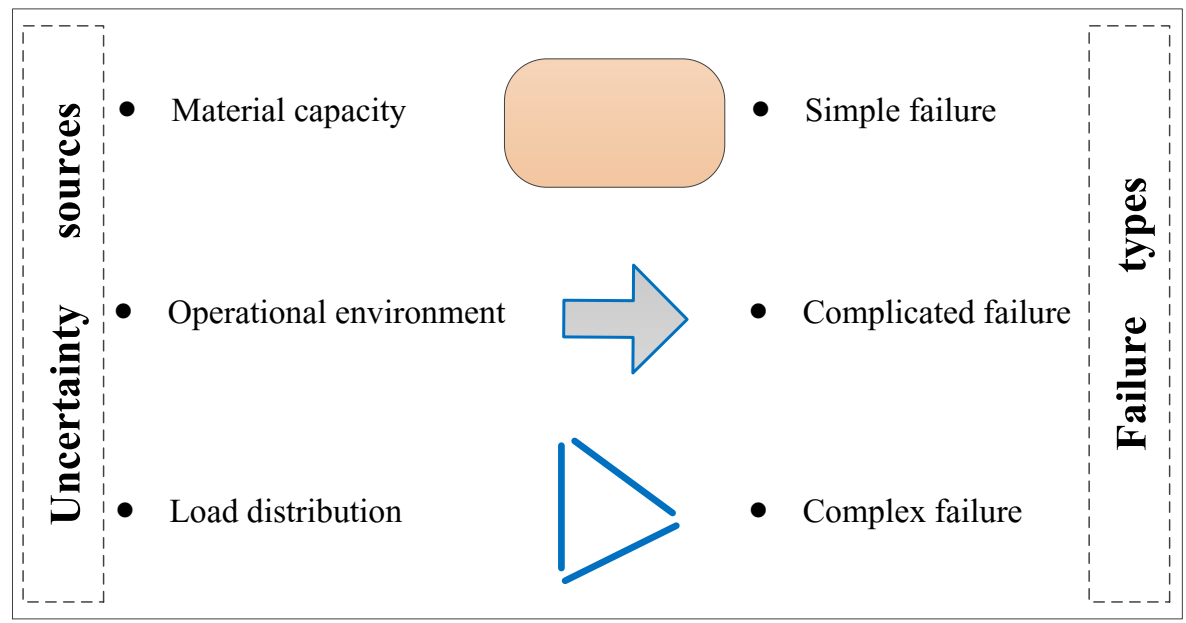

Figure 1.3: Uncertainty sources related to failure types. Icons refer to the elements in Figure 1.2.

its structural collapse. Even more, it was likely that prior the gearbox failure, the resulting abnormal forces propagated throughout the drive train and affected the overall turbine vibration behaviour as shown in the tower acceleration.

From the three categories presented, the complex failure type brings the most uncertainty, and consequently risk, to the overall system. Under complex failure, systems operate with a different load distribution than the originally designed, and consequently all affected components degrade abnormally. It results logical that for avoiding complex failure it is necessary to prevent simple and complicated failures altogether.

\section{Predicting component degradation}

Predicting component degradation under actual operation is not trivial. This requires of reliable component degradation models based on accurate estimations of the component's load history. Specifically, the Physics-of-failure (PoF) approach utilises knowledge of a product's life cycle loading and failure mechanisms as basis of the prediction of component failure. The derived models approximate the component's load history based on the integration of multiple monitored parameters related to the system operation and condition [11]. 
A helicopter landing gear is presented given as Example 2 illustrates the application of PoF on simple component. The component damage was believed to originate from lower material capacity, and hence to correspond to a simple failure type. Yet, the comparison of the seal load history derived from the helicopter actual operation profile proved that the failures developed according to the designed material capacity.

\section{Example 2. Helicopter landing shock absorber [12].}

A premature oil leakage was detected in the landing gear of a fleet of helicopters, for which wear was identified as the responsible failure mechanism. The frequency of the failures did not correspond to the flight hours, which is the common way of determining aircraft maintenance needs.

A degradation model based on Archard's wear law was developed to calculate the volume loss, a wear-specific parameter, from the landing occurrences. This law requires the sliding distance, which depends on both the number of landings and the weight of the helicopter. Using these two, a correlation could be found between specific load parameters and the seal would start to leak. Furthermore, the calculation of material capacity consumption revealed a consistent failure pattern with the manufacturer claims.

A second example of a gas turbine hot section blade is given as Example 3 to illustrate the challenges of predicting component degradation for more complex components. In this case, the material degradation corresponds to a case of failure mechanism coupling, subject to more uncertainties in comparison to the helicopter landing gear example. Additionally, the gas turbine context shows the difficulties to estimate the actual component loading under multiple converging load types (e.g. thermal, mechanical). Also, the case shows the influence of miscellaneous difficult-toquantify aspects such as the maintenance schedules on the actual component degradation.

The gas turbine case also showed the use of previous failure occurrences as an input for the development of damage models. This shows the relevance of empirical knowlegde as element of failure assessment. Although in this example the augmented model led to higher accuracy on the prediction of component failure, in general this approach is limited to cases that follow the same pre-conditions as the failures used for training the damage model. 
These two examples demonstrate that predicting, and hence avoiding component failure for complex components is difficult in practice. Degradation models can lead to accurate predictions for simple loading conditions and under high certainty about the failure mechanisms involved, as seen in the helicopter landing gear case. For more complex systems the component loading history and the failure mechanism models are more difficult to establish, as shown in the gas turbine case. The next section discusses the role of vibration analysis for the characterisation of the load distribution.

\begin{abstract}
Example 3. Gas turbine hot section blade [13].
The PoF model for predicting component consumption of gas turbine hot section blades was developed by the gas turbine manufacturer. The model focused on creep as the main contributing failure mechanism, and used functional models and load history to determine the failure mechanism-stress history. Given the uncertainties for modelling other contributing failure mechanisms such as fatigue and oxidation, the model was calibrated using loading parameter data from failed cases. However the accuracy of the physics-only model did not converge to the observed failures in the training set. A second tuning phase was developed through a machine learning model. The machine learning approach was used to account for qualitative parameters regarding the operational environment known to be relevant for the component lifetime consumption, yet difficult to model from a physics perspective. The augmented model displayed higher prediction accuracy.
\end{abstract}

\title{
1.3 Vibration analysis
}

Vibration behaviour is a natural indicator of the system degradation by reflecting the dynamic load distribution. Rotating mechanical systems have an intrinsic vibration profile defined by the interactions of the system's main forces and structural resonances. Variations from the vibration baseline reflect the development of abnormal forces and resonances disrupting the designed system dynamic behaviour. Hence, the analysis of the vibration behaviour contributes to the assessment of a mechanical system's degradation. 


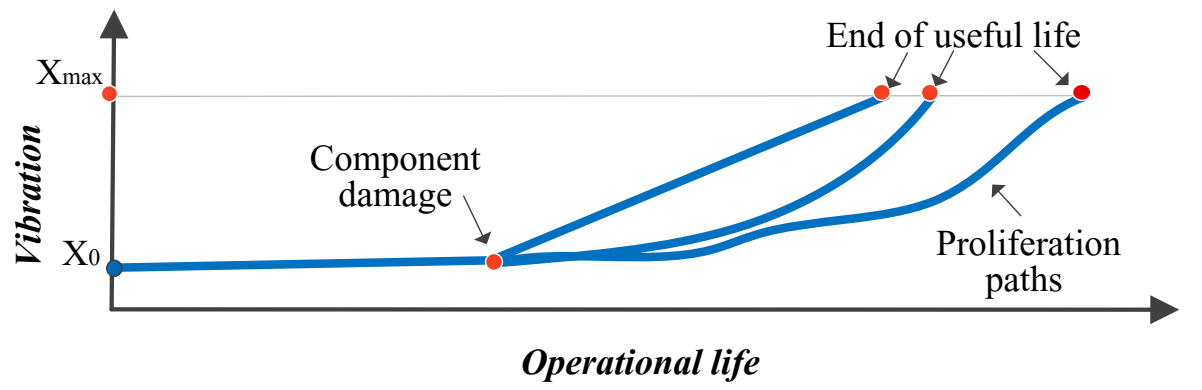

Figure 1.4: Changes in the vibration behaviour under complex failure.

Figure 1.4 illustrates the variations on the vibration behaviour for different moments of the system's operational life. The vibration baseline is represented by a constant line with magnitude $X_{0}$, which is disrupted by the component damage. The subsequent failure proliferation leads to increasingly higher vibration levels up to a maximum value of $X_{\max }$. Three alternative proliferation paths are shown: linear, exponential and escalated. The specific path followed by the failure proliferation defines how fast the system reaches the end of its useful life. The next sections discuss the role of vibration analysis for the characterisation of component damage and failure proliferation.

\subsubsection{Early damage detection}

The application of vibration analysis for early damage detection developed mainly through empirical observations. The initial approximation to vibration analysis consisted in the use of a screwdriver for the isolation of the repetitive, high pitch noises indicative of damage. This acoustic method is also carried with the help of instruments such as a stethoscope, as shown in Figure 1.5a. However, the original version is still very much used. Although highly qualitative, the acoustic assessment is supported by two distinctive features related to abnormal vibration: its repetitive nature and the higher frequency contents involved, compared to the lower pitch of the normal vibration behaviour. This makes acoustics-based assessment a low-cost, yet effective method of detecting common problems such as looseness, poor lubrication and bearing damage. However, this method is highly qualitative and restricted to simple industrial equipment such as pumps and electrical motors. 


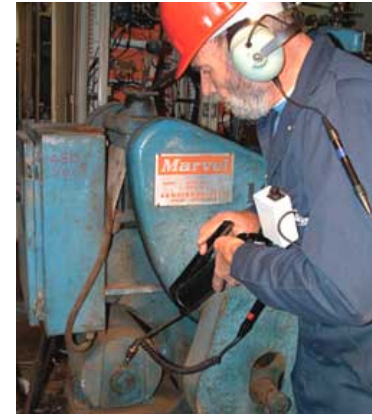

(a) Acoustic analysis

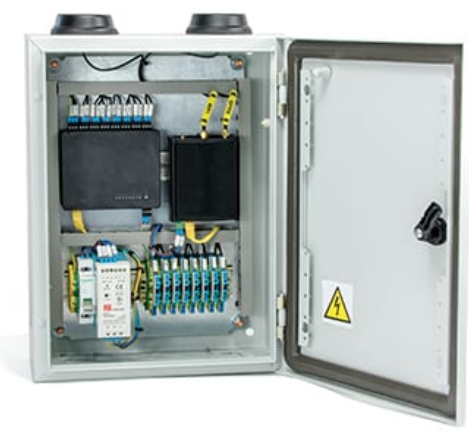

(b) Online Condition monitoring system

Figure 1.5: Acoustic versus vibration analysis in an industrial setting ${ }^{2}$.

The development of the Fast Fourier Transform (FFT) enabled the quantitative assessment of vibration behaviour. Spectral analysis enabled the components of the vibration signal to be matched with a machine's kinematics. Also, the normal vibration response could be mapped, hence it became possible to detect significant deviations from the normal system degradation. Currently, most of the industrial practice of vibration monitoring is based on acceleration measurements acquired with portable collectors or on-line monitoring systems (Figure 1.5b).

As systems become more complex, i.e. flexible operational ranges and greater number of interacting components as in the case of wind turbines, new approaches to vibration analysis are necessary. The wider operational range makes the definition of normal vibration envelopes more difficult. This has been addressed by adopting a statistical approach based on large samples of statistical features (e.g kurtosis, rms) extracted at the main structural frequency ranges of the systems [14]. The greater number of interacting components have been addressed by highly accurate algorithms to track and extract impulse-like singularities representative of damage $[15,16]$.

However, it is argued that combining the low-frequency statistical features and high-frequency damage-oriented features is insufficient for a broader characterisation of the load distribution changes. Imbalance and misalignment are examples of failure modes that are difficult to detect using general statistical features, allowing failure to progress undetected. Furthermore, these types of failure modes are of great importance as they disrupt the overall load distribution to a broader extent, accelerating the simultaneous material degradation of a larger set of system components.

\footnotetext{
${ }^{2}$ Taken from $a$ ) www.machinerylubrication.com and $b$ ) www.chemengonline.com .
} 


\subsubsection{Failure proliferation assessment}

As illustrated in Figure 1.4, the specific path following the component damage defines how fast the system will reach critical degradation. The second part of the wind turbine example illustrates the relevance of vibration assessment for limiting failure proliferation.

\section{Example 1. Wind turbine example. Part II.}

Vibration analysis and endoscopic inspections were carried to identify the most critical damage as part of the wind turbine assessment. The turbines did not have their own condition monitoring system, except for tower vibration sensors. The vibration analysis was carried out by the author of this thesis.

The first part of the vibration analyses focused on general vibration features (i.e. rms values) according to specific industry standards. For the gearbox the recommended frequency range of evaluation is between $10 \mathrm{~Hz}$ and $2000 \mathrm{~Hz}$. Given the highly variable operational environment, multiple measurements at different wind speeds were recorded. Even after all these considerations, most of the overall vibration levels did not raise any significant warning. The second part of the analyses investigated a higher frequency range between $3 \mathrm{kHz}$ and $5 \mathrm{kHz}$. The time waveform analysis showed repetitive impulsive behaviour associated with race damage, as confirmed later by visual inspection.

The overall diagnostics for the entire wind farm showed that almost $70 \%$ of the turbines had faulty bearings. The prioritisation for the turbines at greater risk was defined mostly on the basis of the impulsive responses, as the general vibration features showed little sensitivity to this type of failure.

The wind turbine example illustrates the difficulties of characterising the normal vibration response and the failure proliferation path for system with varying operational environment. The use of the damage features as the main criterion for the turbine's risk assessment gave a suboptimal result, since the high frequency ranges exclude the main gearbox resonances. Yet the poor resolution of the rms values did not capture the variation of the operational forces, as it probably occurred prior the gearbox collapse. 
It will be argued that the interpretation of the vibration behaviour for proliferating failures must be supported on the following aspects of the system dynamics:

(i) the physics of the damage at the component level for each affected component,

(ii) the load distribution associated with the system designed functionality and the major changes expected due to failure, and

(iii) the variations of $i$. and $i$. with respect to operational environment changes.

This means that to monitor the changes in the load distribution associated with the specific proliferation path, the vibration features must be evaluated against an existing model reflecting the system dynamics. However, the simultaneous consideration of these three aspects for the failing system dynamics is rare. Nevertheless, there are multiple examples from analytical, numerical and physical demonstrators that combine some of these aspects.

Analytical models are developed mostly for simple systems to characterise the nonlinear effects of combined failure modes. Numerical models can represent the actual system characteristics more accurately. However, these models are seldom available during the operational phase since they are almost exclusively for design purposes. And even if available, simulating changes in the system behaviour due to damage is not trivial as the accuracy needed to model damage-related nonlinearities raises the modelling complexity. Conversely, physical demonstrators simulate the characteristics of the system dynamics in combination with local damage [17]. Similarly, to the analytical models, physical demonstrators are mainly qualitative while numerical models are more suitable for quantifying the dynamic response.

\section{Data-driven modelling}

In addition to the physics-based models discussed previously, real-life observations support the construction of empirical models of the failure behaviour. Empirical models are based on correlations of observable parameters prior a failure event, which are used for remaining useful life (RUL) estimations. The observed parameters are not restricted to vibration features but include a wide range of sources, including operational data, alarms and maintenance logs.

Statistics-based methods such as machine learning and deep learning are used for the construction of the empirical models for system level failure. Artificial intelligence methods attain high expectations to describe virtually any nonlinear dynamics on the basis of the data alone $[18,19]$. This capacity is deemed highly favourable for dealing with the designed complexity of the rotating mechanical systems (i.e. the number of components and interactions between components) and the additional emerging complexity attributed to failure (i.e. number of failing components and the 
changes in their interactions) [20-24]. The reliance on data alone for the construction of the models is claimed to limit the likelihood of bias on the possible relations drawn by the data analysis [25].

The correlation capabilities of AI have motivated many proponents to consider it as a theory-free basis for new scientific paradigms [26]. Yet, the absence of theory on data-intensive modelling is highly disputable as a level of organising criteria is required at least as guidance for the method implementation. Furthermore, in the context of the maintenance of mechanical systems, the outcomes such as black box, physics-free and human-free modelling approach may not be easily accepted as the only basis for maintenance interventions.

Another important argument in favour of machine learning approaches for the assessment of mechanical systems is the huge amounts of available data given the increased instrumentation of rotating systems. However, it is argued that the reliance on data alone suffices only for highly repetitive failure occurrences. The amount of data required for valid models depends on the capacity of the training data to capture the surrounding conditions, causalities and effects of the failure process $[27,28]$. This means that models require sufficient observations that cover all or most relevant differences between and within systems. Additionally, models are required to account for differences between failures and for the combined effect of multiple failing components. Finally, the actual observations of the end of the system's useful life are rare, as actual systems are seldom allowed to run to failure.

The described conditions restrict empirical models to failures with low uncertainty, i.e., that originate from the same pre-conditions and follow a similar path as the occurrences used to build the model. [20,29,30]. Consequently, the effectiveness of machine learning for prognostics in the general case of complex failure is limited [31-35].

In summary, vibration behaviour analysis holds an untapped potential for the characterisation of the load distribution changes on rotating mechanical systems. The interpretation potential is associated with the integration of the local damage dynamics, the system's dynamic behaviour and operational environment influence. While analytical and numerical models, as well as physical demonstrators, can support some of these aspects, none of these can integrate them all. Empirical datadriven approaches present a modelling alternative embedded on the actual systems context. Although these models are suitable for integrating different sources of information regarding the system behaviour, these approaches alone are insufficient to derive generalisable models of the failure behaviour. In conclusion, the characterisation of load distribution changes associated with complex failure requires new strategies that combine the insights from physics-based models and the deployment opportunities of artificial intelligence modelling. 


\subsection{Thesis goal}

The previous discussions show that failure increases the complexity in the behaviour of rotating mechanical systems and that failure is itself a complex phenomenon. Failure occurs as a combination of uncertainties about the material capacity, load distribution changes and operational environment on top of an already complex web of system functionalities characteristic of rotating mechanical systems. The specific case of complex failure was analysed in detail. Although another two categories of failure were identified, i.e. simple and complicated, the complex type presents the most risks for mechanical systems. Furthermore, it is argued that vibration behaviour reflects of the load distribution changes associated with complex failure, yet a theoretical framework is necessary for its interpretation.

This thesis argues for an increased understanding of the complexity of failure and for better observation of the vibration behaviour as reflection of the load distribution changes. The combination of greater understanding and better observation capabilities is deemed necessary for the development of more effective strategies to limit and even avoid failure and, perhaps of greater importance, for the design of more reliable yet efficient mechanical systems.

Thesis goal: The objective of this thesis is to improve the capacity to understand complex failure and to observe it through the analysis of the associated vibration behaviour.

The realisation of the thesis goal is guided by four research questions, from which the first two focus on the capacity to understand and the remaining ones on the capacity to observe. The first question points to the mechanisms of complex failure, i.e. the why. The concept of mechanism refers to the fundamental processes responsible for the abnormal degradation. This implies the causes that turn simple and complicated failure into complex failure as well as the successive reorganisations of the system load distribution. This is formulated as:

1. What are the mechanisms that explain complex failures?

The second question addresses the dynamic development in time, i.e. the how. The dynamics concept emphasises the continuous load distribution changes under complex failure. Specifically, the question relates to the driving factors, i.e. principles, that determine the degradation rate of a system, and whether to expect that changes in the load distribution remain quantitative or rather qualitative at a given time of the failure development. This is formulated as:

2. What are the principles that predict the complex failure dynamics? 
The third question focuses on the vibration behaviour directly, and hence the features requirements for its interpretation, i.e. the method. Such features must derive from the nature of the different dynamic forces and the resonances reflected on the vibration signal, as well as the expected nonlinear changes in system behaviour. This is formulated as:

3. What are the features of the vibration response that enable the interpretation of load distribution changes associated with complex failure?

The last question addresses the necessary conditions to be considered for adequately registering the vibration behaviour, i.e. the instruments. These conditions are intended to guide the design of the monitoring systems. The last question is formulated as:

4. What are the requirements for the design of vibration monitoring systems for the registration of load distribution changes?

\subsection{Approach}

This thesis addresses the phenomenon of failure from the combination of two approaches based on complexity theory $[3,36]$. The first approach recognises the designed complexity of the object system, also known as time invariant complexity. The second approach focuses on the emerging complexity as a consequence of failure, also known as time variant complexity.

\subsubsection{Designed complexity}

Based on Lee's definition of complexity [2], the difficulty of understanding a system derives from the interrelationships between its elements. The realisation of systems with a high designed complexity is enabled by exposing and managing the interdependencies that give rise to synergistic functional behaviour.

Conversely, in the context of failure assessment, the concept of interdependencies is addressed to a limited extent. Current physics and empirical modelling approaches are restricted to individual failure mechanisms and failure modes.

To explain how component interdependencies enable the onset and proliferation of failure, this thesis adheres to systems thinking as used in the design phase. To a certain extent, the failure assessment is inverse to the design. According to Gero [37] the meta-goal of design is to transform requirements which embody the expectations of the purposes of the resulting artefact into design descriptions of the constituent 
parts and their relations. In this sense, the goal of failure assessment is to evaluate the extent to which the system requirements are no longer satisfied by the effect of changes on the constituent parts and their relations.

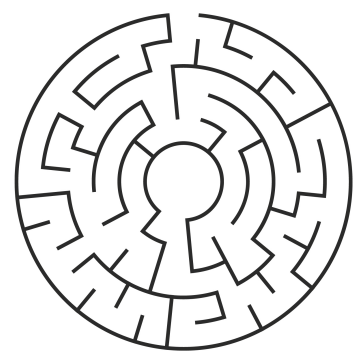

The designed complexity is represented by a circular labyrinth. As stated before, the designed complexity of rotating mechanical systems is reflected in the difficulty of achieving an overall advantageous set of functions through a strategic configuration that satisfies the most important requirements.

Figure 1.6: Representation of the designed complexity.

Based on the commonalities between conceptual design and failure assessment, a formulation of the Function, Behaviour and Structure (FBS) aspects of the failing system is used. Figure 1.7 presents a comparison of the description of system and failure based on Gero's FBS formulation [38].

As discussed before, current failure assessment approaches are based on the material degradation while vibration diagnostics focuses on the physical characteristics of damage. By adopting a function and behaviour description of the failing system, this thesis aims to create more effective links that extend the failure discussion beyond the structural aspects. These links are expected to lead to a better understanding of the interdependencies that cause material and functional degradation.

\subsubsection{Emerging complexity}

The second approach used to study failure refers to the emerging complexity. One of the essential characteristics of complex systems is their collective behaviour which is not readily understandable from individual components properties [2]. For instance, the natural vibration resonance modes cannot be derived from the components' individual dynamic properties. For systems undergoing complex failure new emerging behaviours appear. Such behaviours differ from the designed behaviour as they result from reorganisation of the load distribution. Once the designed equilibrium is disrupted, it becomes replaced not by one but by a stream of new emerging behaviours ascribed to the proliferation of failure. 
Design
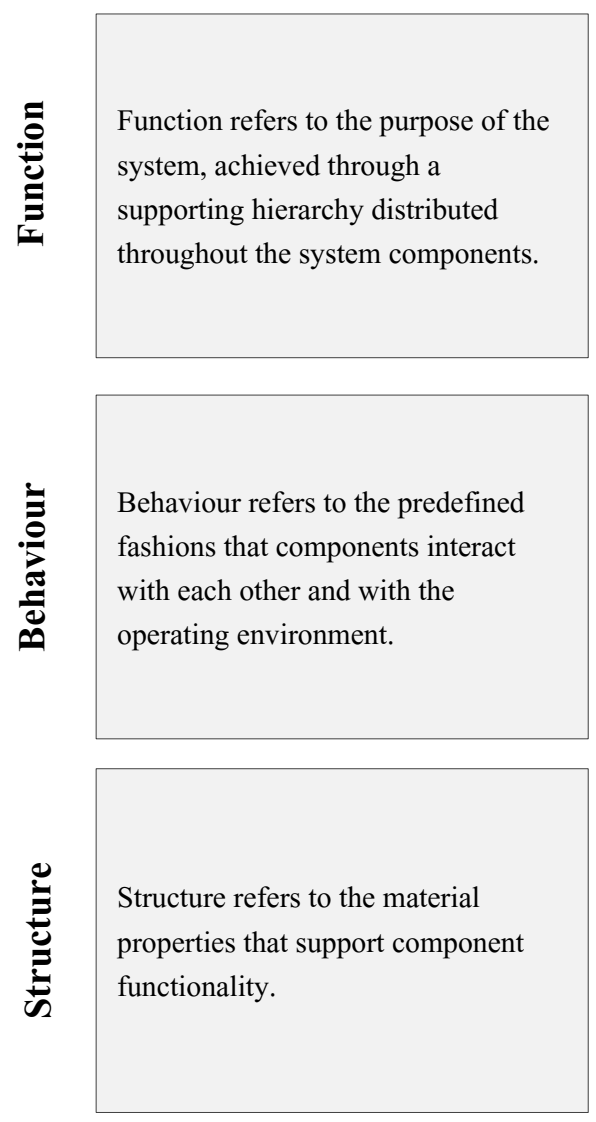

Failure

As each component contributes distinctively to the system's functionality, a specific component failure also carries different effects to the system performance and reliability.

Failure challenges the synergistic interactions of components.

Component failure arises from individual or interacting failure mechanisms degrading the material properties in ways that affect the prescribed functionality.

Figure 1.7: FBS descriptions in the design and failure contexts.

This implies that in addition to the complexity of the system, the phenomenon of failure is also recognised as complex. In contrast to the designed complexity where the behaviour is motivated by the functional requirements of the system, for the emerging complexity the arising behaviours derive from the interactions of the failing components. 
The emerging complexity is represented by a spiral. Complex failure emerges unexpectedly and unpredictably as a result of a new type of functional dependency, different from those responsible for the designed complexity. Consequently, the resulting emerging complexity is guided by principles different from those guiding the design of the engineered system.

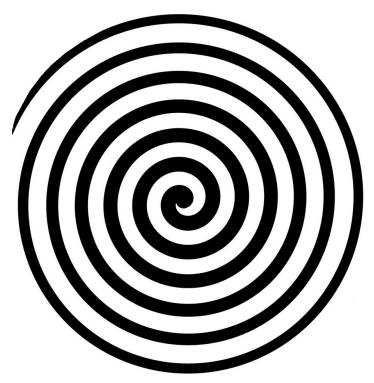

Figure 1.8: Representation of the designed complexity.

Unlike the time-invariance of the designed complexity, the emerging complexity is time-dependent. This occurs as a consequence of future events not considered in the design phase that affect the system in unpredictable ways. Often this results in a time-varying system range, i.e. the system range moves away from the design range. Complexity theory addresses the study of time-dependent complexity through a set of concepts (i.e. chaos theory, feedback loops, exponential growth, selforganisation) [39] that explain how simple nonlinear interaction convolve to generate complex behaviour. The application of complexity theory in this thesis focuses on the characterisation of the failure behaviour.

Figure 1.9 illustrates the extent the system behaviour changes due to failure. The designed behaviour (i) is represented by a vertical black line. As a consequence of the changes in the operational environment, the designed behaviour displays slight deviations, as shown by the grey lines around the vertical position. As result of failure, the system behaviour deviates from the designed balance, as represented by the blue line, while the functional effects are represented by the red arrow. Initially moderate variations from the designed behaviour reflect on a linearly divergent failure behaviour (ii to iv). Yet as the divergence intensifies, new and more intense failure states emerge $(\mathrm{v})$, leading to more significant effects on the system functionality.

In sum, the application of complexity theory to failure of rotating mechanical systems is expected to provide insights into the principles that drive the divergent and progressive changes of the failure behaviour.

\subsection{Contributions}

These questions addressed in this thesis correspond to a first step towards the construction of a theoretical basis for the study of system-level failure of rotating ma- 


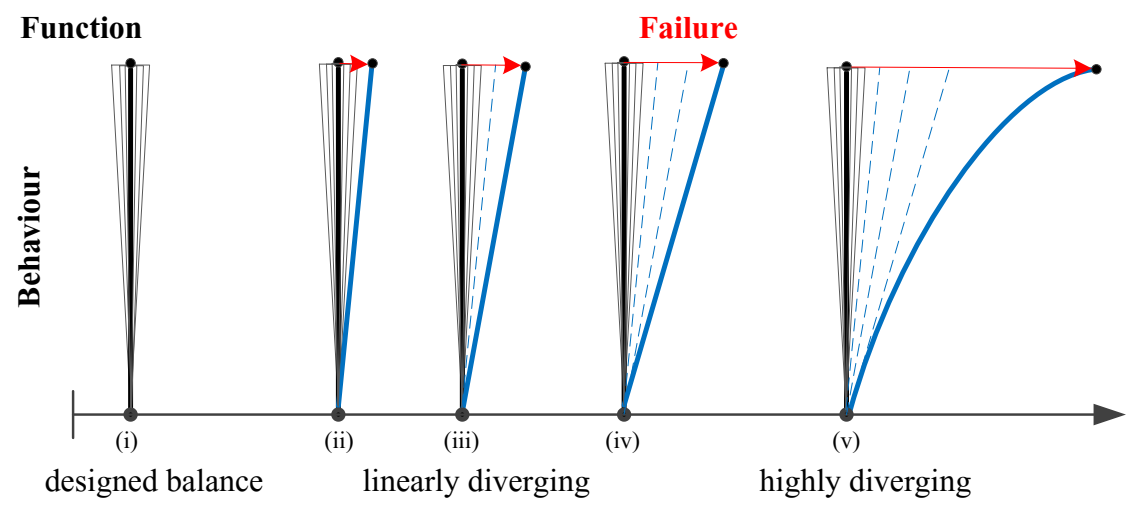

Figure 1.9: System behaviour change due to failure.

chines. The main overarching goal is that although failure cannot be entirely avoided, its complexity can be managed. But this requires a continuous observation of the actual changes in the system behaviour, for which vibration analysis addresses the dynamic aspects only. Thus a more comprehensive approach that considers the thermal, chemical and electromagnetic aspects must also need to be considered for a general theory.

Nevertheless, this thesis addresses two theoretical levels. First, the hypothesisgeneration about the most likely causes and evolution of the failure development for a given system. And second, the general guidelines for the organisation of the observation itself. These ambitions are reflected in four distinctive contributions as shown next.

Material to system level: As explained in the previous section, the function and behaviour aspects of the failing system are developed to complement the current focus on the structural aspect of damage diagnostics. This leads to better characterisation of the load at the component level as support for PoF models and provides a better assessment of the implications of component damage on system functionality.

From predicting remaining useful life to studying a failing system: Whereas the goal of empirical and failure mechanism-based models is the prediction of a future state, the study of load distribution changes assesses the current state of the 
system. The shift in goal offers more opportunities for timely and effective interventions that correct load distribution deviations.

From algorithms to features: Most of the current focus of the academic discussion on vibration analysis is on the methods for signal processing algorithms and statistical modelling. This thesis presents a comprehensive description of the most relevant aspects of the vibration response and how they relate to the load distribution changes. The discussion on features presents a guiding criteria for algorithms development and method selection.

Monitoring systems able to register load distribution: Finally, the thesis synthesises the insights into the failure phenomenon and the vibration behaviour as requirements for the monitoring systems. This is presented as a design framework for the integration of the monitored and monitoring system.

\subsection{Thesis outline}

The thesis is organised in three parts as shown in Figure 1.10. Following this introductory chapter, the first part develops conceptually on the complexity of failure. Chapter 2 addresses the mechanism that enables the onset and proliferation of failure. Chapter 3 addresses the dynamics of failure and postulates three principles for the characterisation of complex failure behaviour.

The second part focuses on the functional approach to vibration analysis as guided by the three principles defined previously. Chapter 4 discusses the main elements for the interpretation of vibration behaviour in accordance with the loss energy principle. This chapter lays the methodological basis for the case studies presented in the next two chapters. Chapter 5 addresses the changes in the system behaviour for a given failure state in accordance with the kinetic energy principle. This is developed through a comparative case study of three different systems displaying bearing failure, which differ in the types of damage and the operational environment. A complement to this chapter examining more deeply the technical aspects of the signal processing treatment is presented in Appendix A.

Chapter 6 examines the emergence of new failure states based on the synergies of the constituent failure modes in accordance with the mode-state dependency principle. This is treated on the basis of an experimental set up representing the operational excitations and boundary conditions of a rotor-blade system. Further information related to the experimental set up is presented in Appendix B. 


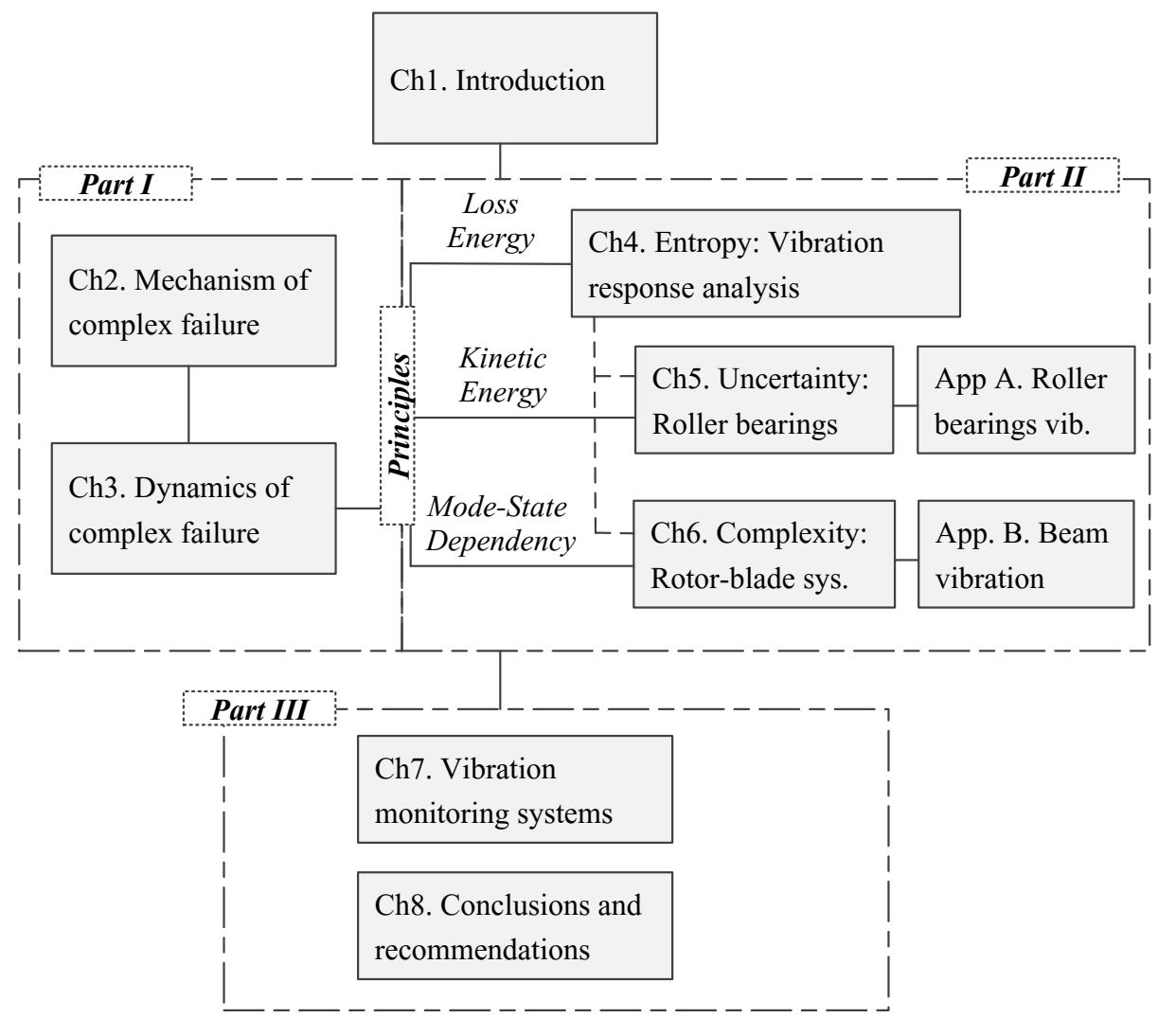

Figure 1.10: Thesis structure.

The final part presents the reflections of the two previous parts for the monitored and monitoring systems. Chapter 7 discusses the design implications for a function based vibration monitoring systems. Furthermore, it discusses the most relevant design requirements for a distributed monitoring system. Chapter 8 presents the main conclusions and recommendations of the thesis. 


\section{Part I}

\section{On Failure Complexity}





\section{Chapter 2}

\section{Mechanism of complex failure}

The concept of complex failure was introduced to describe the major changes in the load distribution responsible of heterogeneous degradation and interdependent failing components. The main limitation to the understanding of complex failure was attributed to the inadequacy of the current physics models to explain failure at the system level. This chapter addresses the mechanism behind complex failure. Based on the definition in Merriam-Webster, the concept mechanism refers to the fundamental processes involved in or responsible for an action, reaction, or other natural phenomenon. Hence, in the context of rotating mechanical systems, the mechanisms of complex failure refer to the fundamental processes responsible for major changes in the load distribution.

This chapter introduces the changes in the functional dependencies, specifically those regarding performance and reliability parameters, as main causes of the onset and proliferation of failure. It will be demonstrated how failure changes the strength of functional interdependencies beyond the designed expectations, and how new states of the system emerge as a consequence of the progressive alteration of such dependencies.

The chapter is organised as follows. Section 2.1 presents an overview of the functional dependencies identified within component boundaries, and those related to the propagation of failing components. Section 2.2 explains the dependency between performance and reliability parameters, and how such dependencies are affected by failure. Next, the implications of these dependencies at the system level are elaborated in Section 2.3. The concept of failure state is elaborated in section 2.4. Finally, conclusions are presented in Section 2.5. 


\subsection{Background}

In Chapter 1 complexity was defined as the property of a system that makes it difficult to understand as a whole through the collection of knowledge about its constituents. Such difficulty is attributed partly to the interdependencies exhibited among the components. Yet dependencies and interactions cannot be regarded only as a negative feature of the system that limits the capacity to understand it. The efficiency of complex systems is very much associated with the high synergy achieved by the design. Synergy is understood to mean the extent that by achieving requirement $a$ a second requirement $b$ is facilitated.

The problem of dependencies arises when there is a conflict between requirements. Following the previous example, it will not be possible to achieve full satisfaction of requirement $b$ while achieving requirement $a$. Although minimizing possible negative interdependencies is a core task in design, this cannot be always guaranteed fully given the remaining uncertainty in the functional relations between requirements.

This section presents an overview of the known dependencies associated with component failure and failure proliferation. Such dependencies are discussed in relation to how they affect the material, component and system level. A representation of the main topics discussed in this section is presented in Figure 2.1.

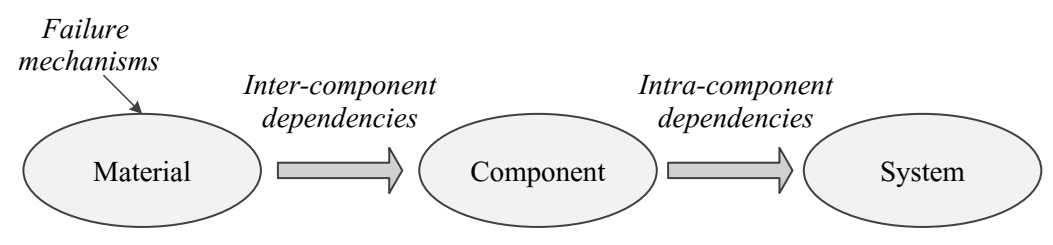

Figure 2.1: Dependencies at the material, component and system level.

The section is organised as follows. Section 2.1.1 presents a classification on the effect of failure mechanisms according to the effects on the relation degradationstresses within the material. Section 2.1.2 introduces the intra-component within dependencies that define the effect on component functionality, while Section 2.1.3 focuses on the inter-component between dependencies that define the progression on failing components. 


\subsubsection{Failure mechanisms}

Failure mechanisms refer to the physics processes responsible for the release of the potential energy stored within the component and at its boundaries. In a general sense, potential energy relates to the capacity of an entity to undergo a change in its configuration. For instance, in a mass-spring system the potential energy is the stored energy that the system can convert to kinetic energy due to a previous elongation (configuration) that determine the system's instantaneous position. Similarly, materials bury potential energy in the crystalline structure, electric charge and chemical bonds. As a consequence of the failure mechanisms, materials undergo changes in their macro- and micro-properties [40], with undesirable effects on the functionality of the component they form.

Failure mechanisms can be classified according to the relation degradation-stresses. Dasgupta identifies wear-out and overstress types of mechanisms [41]. For wear-out type a stress causes damage that accumulates irreversibly. Thus the component design must be such that the function degradation occurs only until the endurance of the material is reached. For overstress type, damage occurs only if the stress exceeds the material strength as in the case of brittle fracture and buckling. Additionally failure mechanism can be classified according to whether they affect the bulk material or the component boundaries. In the Table 2.1 a mapping of failure mechanisms according to the two classification types proposed is shown.

Table 2.1: Classification of failure mechanisms according to the degradation-stress relation and the area of influence.

\begin{tabular}{|l|l|}
\hline \multicolumn{1}{|c|}{ Overstress - Bulk } & \multicolumn{1}{c|}{ Wear-out - Bulk } \\
\hline $\begin{array}{l}\text { Fracture (brittle and ductile) } \\
\text { Yield } \\
\text { Buckling } \\
\text { Large Elastic deformation }\end{array}$ & $\begin{array}{l}\text { Fatigue crack propagation } \\
\text { Fatigue crack initiation } \\
\text { Creep }\end{array}$ \\
\hline \multicolumn{1}{|c|}{ Overstress- Boundary } & \multicolumn{1}{|c|}{ Wear-out - Boundary } \\
\hline & $\begin{array}{l}\text { Wear } \\
\text { Corrosion } \\
\text { Dendritic Growth } \\
\text { Infer-facial de-adhesion }\end{array}$ \\
& $\begin{array}{l}\text { Inter-Diffusion } \\
\text { Radiation }\end{array}$ \\
\hline
\end{tabular}

Given the multiple types of loads the components are exposed to, the constituent 


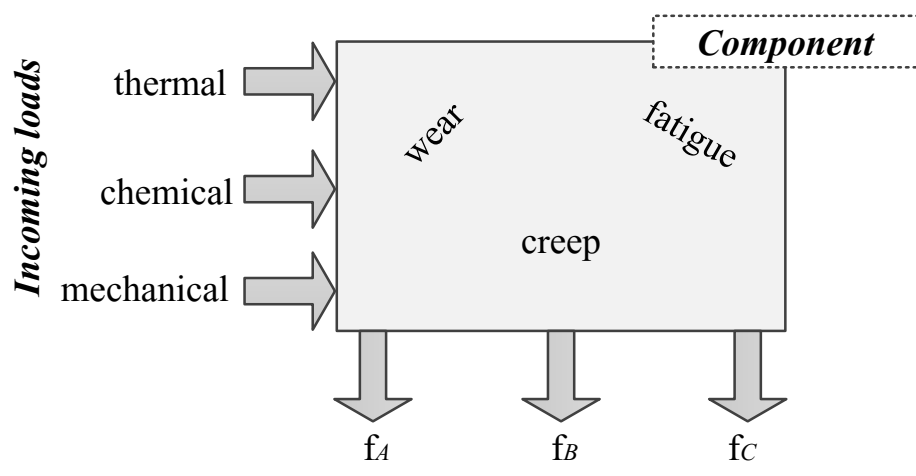

Functional requirements

Figure 2.2: Representation of complexity at component-level

materials are often subject to multiple failure mechanisms that together define the component capacity.

\subsubsection{Intra-component dependencies}

Component failure is regarded as the result of an imbalance between the load on the component and that systems load-carrying capacity [42]. However, most components are exposed to more than one type of load, display more than merely failure mechanism and are designed to meet more than one functional requirement. In line with the previous definition of complexity, components exhibit high design complexity when failure mechanisms, incoming loads and functional parameters interact within the boundaries of the component, as illustrated in Figure 2.2.

The multiplicity of failure mechanism, incoming load types and functional parameters leads to multiple types of interactions likely to occur in complex components. These interactions are failure coupling, competition, superposition and collaboration, as illustrated by the interaction graphs in Figure 2.3. The interaction graphs represent the interdependencies (blue lines) between the incoming load, the active failure mechanism $f m$ and the functional parameter $P$ affected.

Failure mechanisms are (structurally) independent when individual mechanisms occur without affecting each other. Conversely, failure mechanisms exhibit coupling when the physics process become intertwined as the materials are exposed simul- 


\section{Incoming}

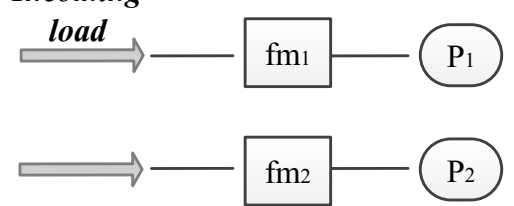

a) Independent

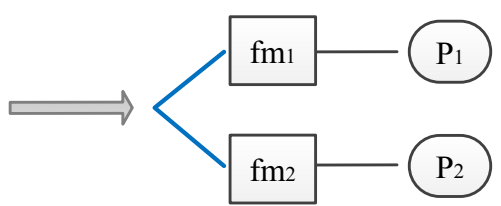

b) Competition

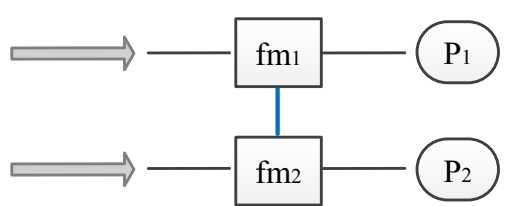

c) Coupling

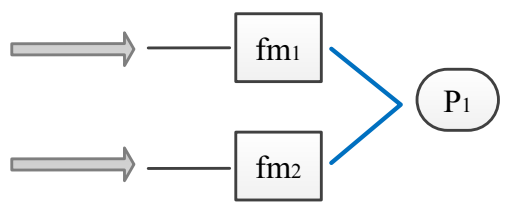

d) Superposition

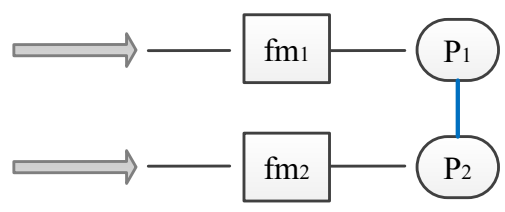

e) Collaboration

Figure 2.3: Interaction graphs for intra-component dependencies ${ }^{1}$

taneously to different load types. In general, the physics of failure mechanism coupling is highly dependent on the physics of the failure mechanism itself and on the environmental operation ranges in which the system operates [43].

Fatigue is a prototypical example of coupling, as the physics of crack initiation and growth can be modulated by diverse factors [45]. For instance, the combination with fatigue and overload can lead to crack retardation. Additionally, fatigue crack initiation can be exacerbated by the presence of corrosion pits. Yet in some cases independent failure mechanisms display a coupling when operating under an environment (e.g. salinity, temperature) other than the optimal operational range, as in the case of creep and fatigue.

\footnotetext{
${ }^{1}$ Modified from Zeng et al [44].
} 
Failure mechanisms are functionally independent when they are affected by different load types and they contribute to distinct component functions [41]. Yet other types of functional interaction occur when failure mechanisms are affected by the same load or contribute to the same key performance indicator, even in absence of actual physics coupling [46]. For instance, in the case of competition multiple failure mechanisms trigger different failure modes yet the presence of one has little or no influence on the others. The failure of components undergoing competing failure mechanisms is determined by the fastest degradation, i.e. the weakest link.

Another case is the superposition of failure mechanisms, where simultaneous failure mechanisms contribute to the degradation of one performance parameter. In this case the overall degradation is determined by the summation of each individual failure mechanism contribution. Although the presence of competition or degradation must be anticipated at design, the actual participation - given the unique load history of the components - holds greater uncertainty than in the case of independent failure mechanisms.

Finally, the case of failure collaboration corresponds to the joint effect of individual functional degradation on the overall system degradation. This concept highlights that as components collaborate with each other in coordinated fashions defining the system behaviour; likewise, the individual degradation of components leads to a coordinated effect on the overall system's failure behaviour. This case is illustrated in the case of a hydraulic servo actuator, as shown in Example 4.

The concept failure collaboration emphasises that system performance degrades even when the individual components degrade in accordance with their expected useful life [7]. The distinction between performance and useful life as different aspects of the system functionality is key to the onset of complex failure, as will be addressed later in Section 2.2. In sum, the presence of interdependencies at the structure and function level increases the chances of abnormal or accelerated system degradation. 


\section{Example 4. Failure behaviour of a Hydraulic Servo Actuator [47].}

The failure behaviour of a Hydraulic Servo Actuator (HSA) considering failure collaboration is compared with the traditional PoF-based approach based on the independence assumption. The HSA's main function is to transform the input electrical signals into the displacement of the hydraulic cylinder (HC). The selected performance parameter is the attenuation ratio between the $\mathrm{HC}$ displacements to the objective value. This means that the system fails when the attenuation ratio reaches a critical value. HSA degradation is calculated based on Physical Functional Models (PFM) that describe the contribution of individual component functionality to the system function, the degradation sensitive parameters for each component and the failure mechanisms that influence the degradation sensitive parameters.

The traditional PoF method estimates the overall system TTF as the shortest TTF among the components. The failure collaboration model considering the joint effect of individual component deterioration leads to a system TTF shorter than the TTF derived from PoF. This means that the model considering failure collaboration results in a more realistic prediction of the systems failure behaviour.

\subsubsection{Inter-component dependencies}

Failure propagation refers to the trajectory of subsequent degradation of system components after an initial trigger event. Three scenarios of propagation are identified that refer to when failure occurs either simultaneously or sequentially, and whether the component degradation occurs discretely or progressively (Figure 2.4).

Common-Cause Failures (CCF): simultaneous failure events as a consequence of a common cause, for instance due to operation beyond design limits or due to a natural event, such as an earthquake $[48,49]$.

Cascading Failures (CF): sequential events when the failure at one component propagates progressively throughout the system, i.e. the domino effect [50,51].

Escalating Failures (EF): gradual and overlapping progression of failure throughout affected components [52,53], i.e. accelerated degradation. 
Common Cause

Cascade

Escalating
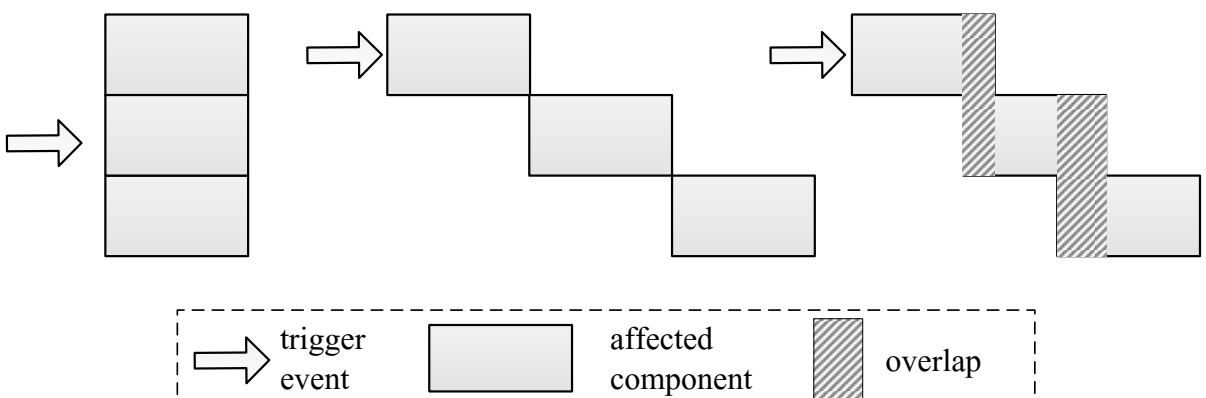

affected component

Figure 2.4: Types of failure propagation

The prediction of the most likely propagation scenarios is necessary to limit risks of shout downs and catastrophic damage $[54,55]$. The estimation of the most likely failure sequence is carried by risk analysis models based on the functional relations between the components. This means that the likelihood of a failed component affecting other component is greater when they have close functional ties. However, the unknown functional dependencies, unavoidable for complex systems, can bring about unexpected scenarios on the failure progression [56]. The relevance of the path estimation concerns complex networks such as power transmission systems $[57,58]$.

In addition to the propagation scenarios risk analysis models must also estimate the degree to which the failure of one component affects other components' functionality for the escalating case. Most modelling of escalating failures has been based largely on stochastic estimators $[52,53,59,60]$, yet few studies have opted for quantification of component degradation rates by using real-time degradation signals [61] and condition monitoring data [62].

The previous two sections presented an overview of the dependencies within and between components that facilitate the onset and proliferation of complex failure. They increase system complexity by adding additional uncertainty about the interactions between the functional specifications. Although these conditions may not necessarily trigger abnormal degradation if estimated correctly, they narrow the operation ranges of the system that guarantee normal ageing. For instance, under failure mechanism coupling material degradation accelerates at a faster pace than in the un-coupled situation. 


\subsection{Performance and reliability dependencies}

The previous section introduced several dependencies associated with unexpected component failure and the proliferation of failure. These dependencies are intrinsic to the designed system as their existence i due to the designed structural configuration and functional relations. This section presents an additional type of dependency that is modified by failure. This refers to the relation between performance and reliability parameters.

Two types of functional requirements (functions) can be distinguished: performance requirements $f_{p}$ relate directly to the main function of the component and system and reliability requirements $f_{r}$ relate to the expected useful life of the component. A spring is used to illustrate the distinction between performance and reliability parameters (See Figure 2.5).

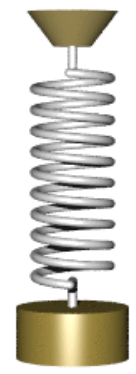

(a) Mass - spring system

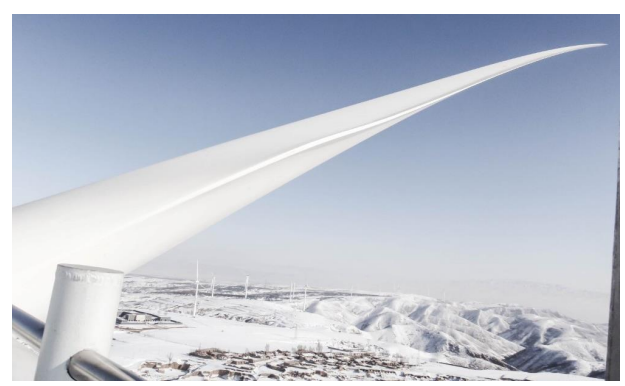

(b) Composite blade

Figure 2.5: Stiffness role on component functionality.

The spring's main function is to restrict the deflection of a part $\left(f_{p}\right)$ during a specified number of cycles $\left(f_{r}\right)$. To fulfil these specifications, the material micro-structure characteristics must conform to the required stiffness and the fatigue load-carrying capacity respectively. As most spring elements are made of metallic materials the stiffness and fatigue life depend on distinct micro-properties (Figure 2.5a). In contrast to composite materials (Figure 2.5b), stiffness and fatigue life exhibit a closer dependency [63].

The different possible dependencies between performance and reliability parameters and the effect of failure in such dependencies are discussed next. 


\subsubsection{Designed dependency}

The designed dependency between performance and reliability functions refers to the extent to which the component performance is affected by the depletion of its capacity. Explicitly, this relates to the dependency of performance and reliability requirements on the properties of the constituent materials. Two cases are identified here. For the independent case - stiffness and fatigue in the metal spring example the performance and reliability requirements depend on distinct material properties. For the dependent case, the two types of requirements depend on at least one common material property, as in the composite case.

The dependency types between performance and the reliability parameters are explained through Figure 2.6. The diagrams illustrate the relations between material properties $m$ that support $f_{p}$ and $f_{r}$ and the overall system/component functionality $T$. Note that these diagrams differ from the interaction graphs shown in Figure. 2.3.

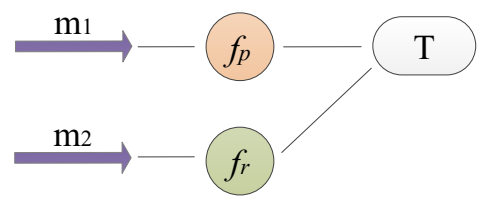

(a) Independent

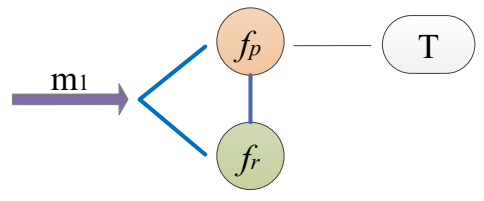

(b) Dependent

Figure 2.6: Performance $\left(f_{p}\right)$ and reliability $\left(f_{r}\right)$ dependency for the main component function $(T)$.

The independence between $f_{p}$ and $f_{r}$ is a desired attribute for system determinism [64]. In the case of dependency, if the component useful life is restricted to avoid load distribution, it changes due to decreased functional performance. In both cases systems are restrained to operate at a linear range that limits the effect of consumption material capacity.

\subsubsection{Failure-induced dependencies}

As mentioned before, systems are designed to operate within linear ranges that reduce the influence of material degradation on system performance. Unintended operation away from this range implies a change in the designed relations between $f_{p}$ and $f_{r}$. For the designed independent case, the parameters become related. For the designed dependent case, the existing relations exacerbate. The differences in degradation within and beyond the linear range are illustrated in Figure 2.7. 
Failure-induced dependencies provide the missing link between the material degradation and the changes in load distribution displayed in complex failure. Figure 2.8 illustrates the onset and proliferation of failure. The figure shows three components $\mathrm{A}, \mathrm{B}$ and $\mathrm{C}$ within the system boundary, and each component has a distinctive composition of $f_{p}$ and $f_{r}$.

Independent $f_{p}$ and $f_{r}$

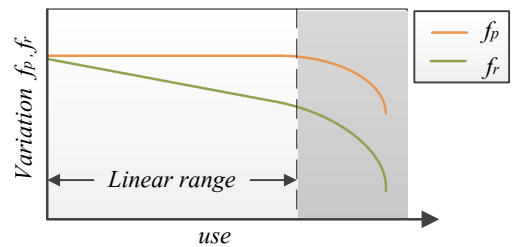

(a) Failure induced dependency
Dependent $f_{p}$ and $f_{r}$

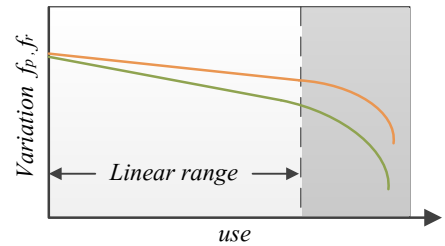

(b) Failure exacerbated dependency

Figure 2.7: Influence of failure on performance $\left(f_{p}\right)$ and reliability $\left(f_{r}\right)$ dependency.

The failure initiates due to a trigger event: either lower component capacity (simple failure) or abnormal operating environment (complicated failure). This causes an accelerated degradation of $f_{r}$ at component A. Beyond the component linear range, the dependency between $f_{p}$ and $f_{r}$ increases. The component performance decreases $f_{p}$.

Failure proliferation starts with the change in the load distribution. As $f_{p}$ is associated with the system main function, it also determines the relation with other components. The load distribution of the entire system changes because of the decreased performance of component A. However, the effects on other components is not necessarily the same, as it depends to the degree of functional dependency between components. In the presented example component A exhibits a higher dependency on component $B$ than on component $C$. This leads to subsequent deterioration of component B.

\subsection{Rotating mechanical systems functionality}

Establishing failure-induced dependency as the main mechanism for the onset of complex failure constitutes an important step towards modelling failure behaviour. As illustrated in the case of the hydraulic servo actuator (Example 4), achieving realistic predictions of system degradation is not trivial, even for a relatively simple 


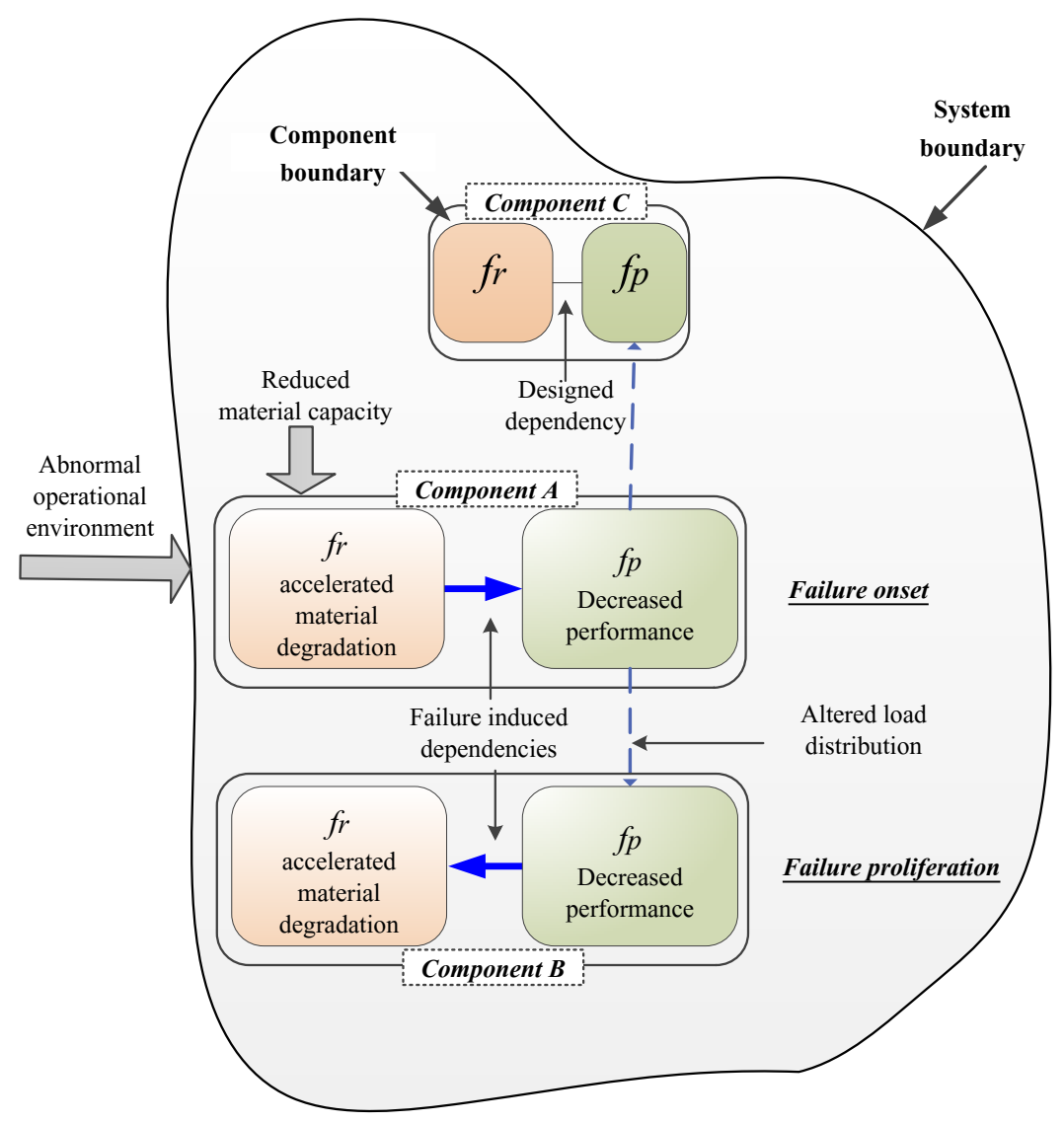

Figure 2.8: Complex failure onset and proliferation. 
component. For the case of complex systems as rotating machines undergoing complex failure, such an ambition seems even more difficult to attain. However, by distinguishing performance and reliability as two different aspects of the system functionality, a new approach opens up for modelling failure behaviour based on the relations between the constituent functions.

This section proposes a first step towards the modelling of failure behaviour based on the different hierarchies and relations typical of rotating mechanical systems. The presented derivations are elaborated from the discussion on performance and reliability dependencies at the component level for achieving a system level description of the phenomenon of complex failure. A series of expressions are introduced for the description of the most relevant functional aspects of rotating mechanical systems. Although the expressions resemble mathematical equations, they are not intended to provide formal guidance for the operationalisation of system functionality, or for the effect of failure. This implies that some symbols are given a specific meaning that is applicable only for this thesis.

An example of the proposed notation is given with the definition of failure by Russell L. Ackoff': 'A system is not the sum of its parts, but the product of the interactions of those parts.' This definition is expressed as

$$
T=\prod_{C} f
$$

where $T$ refers to the system, $C$ to the parts or components and $f$ to the individual functions, while the multiplication sign denotes interaction of individual component functions.

$$
\prod: \text { interaction }
$$

\footnotetext{
${ }^{2}$ Russell Lincoln Ackoff (February 12, 1919 October 29, 2009) was an American organisational theorist, consultant, and Anheuser-Busch Professor Emeritus of Management Science at the Wharton School, University of Pennsylvania. Ackoff was a pioneer in the field of operations research, systems thinking and management science. Source: Wikipedia
} 
The choice of the multiplicative association is illustrative and therefore cannot be taken literally as the complexity of the intended functions cannot be modelled by only one interaction type. However, for the purposes of the current discussion the multiplicative notation emphasises the simultaneous action of the constitutive functions in order to achieve full system functionality. As an example, consider the function of a gearbox transmission where the total transmission ratio is obtained by the multiplication of the individual ratios of all the intermediate stages.

Considering the designed dependencies between performance and reliability parameters discussed in Section 2.2.1, the Equation 2.1 is rewritten as

$$
T=\prod_{C} f_{p} \times f_{r}
$$

where the symbol $\times$ denotes the designed dependencies

The main function of rotational mechanical systems $T$ is to transformation an energy resource extracted from an input state into an purposeful task delivered to an output state. For instance, the main purpose of a wind turbine is to transform the energy content available in the wind resource into electrical power with defined voltage and current specifications compatible with the energy grid. This is presented as:

$$
T_{0}=\frac{S_{\text {out }}}{S_{\text {in }}}
$$

where $T_{0}$ is a transfer function representing the designed system, $S_{i n}$ the input state and $S_{\text {out }}$ the output state.

Performance and reliability are chosen as two criteria for the evaluation of the overall system functionality.

$$
\operatorname{Eval}(T)=\{\text { Performance, Reliability }\}
$$

where performance evaluates the quality of the tasks these are required to the state transformation, and reliability evaluates the endurance (in time) of the system functionality.

$$
\begin{array}{r}
\operatorname{Eval}_{1}(T)=\text { Performance } \\
\operatorname{Eval}_{2}(T)=\text { Reliability }
\end{array}
$$

The next sections explain the evaluation of rotating mechanical system in relation to performance and reliability and how these evaluations derive from $f_{p}$ and $f_{r}$. 


\subsubsection{Performance}

Performance has been introduced as referring to the quality of the tasks that need to be done. For general purposes, performance is defined as energy efficiency of the state transformation,

$$
\text { Performance }=\frac{\operatorname{Energy}\left(S_{\text {out }}\right)}{\operatorname{Energy}\left(S_{\text {in }}\right)}
$$

where the configuration $C$ for the designed state $T_{0}$ is chosen to derive the highest performance possible given the characteristics of the input and output states.

$$
\text { Performance }=\max \left(\left.C\right|_{S_{\text {in }}, S_{\text {out }}}\right) \text { for } T_{0}
$$

Nevertheless the systems behaviour can undergo controlled changes in response to variations in the operational environment reflected in the input and output states, $S_{\text {in }}$ and $S_{\text {out }}$. Beyond this envelope systems can, however, undergo complicated failure.

The contribution of each performance requirement $f_{p}$ to the system main function is unique. A hierarchy of the different $f_{p}$ roles is presented below:

$$
\text { type }\left(f_{p}\right)=\left\{\begin{array}{l}
\mathbf{I}: \text { Power transmission } \\
\text { II }: \text { Structural support } \\
\text { III }: \text { Control } \\
\text { IV }: \text { Mitigation }
\end{array}\right.
$$

where each type is defined as follows.

- Type I: Power transmission: functions related directly to the energy content involved in the state transformation as the main function of rotating mechanical systems. Mostly these relate to the drive train: engine, rotor, generator, transmission system.

- Type II: Structural support: functions that constrain and distribute the dynamic forces generated from the power transmission. Structural support relates to the distribution of internal loads in order to guarantee strength and stiffness necessary for a stable interaction between the moving frame and the fixed world, e.g. fuselage and bearings. Structural configuration enables the synergistic interactions between components, e.g. Degrees-of-Freedom decoupling [64]. 
- Type III: Control: functions that steer the power transmission according to the variations of the operational environment. This means that control functions organise the system behaviour against operational environment and correct system response through negative feedback.

- Type IV: Mitigation: alleviation measures derived from the power transmission and designed to protection of the structural integrity of the participating elements, e.g. lubrication, lube oil filtering, cooling system, dampers.

In the proposed hierarchy, the power transmission role is presented as the most important role, as it relates directly to the kinetic energy transformation associated with the input and output states (Eq. 2.7). The additional three categories enable the power transmission at the highest energy efficiency for the expected system lifetime. In sum, the coordination of performance requirements prescribes the kinetic energy balance of the system.

The nonfulfilment of the different function types alters the overall system functionality in distinct ways. Consider the case of a whip oil phenomena on the oil lubricant (type IV) associated with the support function of journal bearings (type II). As result of the self-excited phenomenon the stability of the rotating shaft becomes compromised, affecting the power transfer function of the supported generator (type I). In this case the failure progresses over several components before compromising the main system function. Yet failures can affect the system functionality more directly because of an incorrect control input (type III) for example, limiting the system performance directly.

The proposed classification of functions suggests that components with power transformation (type I) and control functions (type III) are likely to have a strong influence on the system performance. This is the case with gears, where the power transmission depends on the contact geometry (i.e. tooth profile, geometry, action line), or the pneumatic guiding system, the regulating role of which depends on the system of valves and tubes and the associated control.

$$
\text { Performance } \approx \prod_{C^{I, I I I}} f_{p}
$$

Although the structural support (type II) and mitigation (type IV) functions participate less directly in the system performance, their effect becomes more significant on system reliability, as discussed below. 


\subsubsection{Reliability}

System reliability has been introduced as the system endurance throughout the expected useful life of the system. In the present context, system reliability is conditioned by the degradation rate of the system configuration.

$$
\text { Reliability }=\frac{d}{d t} T
$$

This means that a lower degradation rate leads to a more reliable system and vice versa. By implementing the definition of system in eq. 2.3 , reliability is defined as

$$
\text { Reliability }=\frac{d}{d t}\left(\prod_{C} f_{p} \times f_{r}\right)
$$

Note that in the proposed context the presented definition differs from the widely accepted stochastic sense, yet it provides the linking to the system functionality.

It will be argued that achieving the expected useful life and the uninterrupted operation of the system depends on three distinct mechanisms: the normal ageing $R_{\text {ageing, }}$ long-term reliability $R_{\text {long }}$ and short-term reliability $R_{\text {short }}$.

$$
\text { Reliability }=f\left(\mathbf{R}_{\text {ageing }}, \mathbf{R}_{\text {long }}, \mathbf{R}_{\text {short }}\right)
$$

The normal ageing $R_{\text {ageing }}$ is defined by the degradation of all the reliability parameters.

$$
\mathrm{R}_{\text {ageing }}=\prod_{C} \frac{d}{d t} f_{r}
$$

where the rate of degradation of $f_{r}$ for the main components is bounded by a threshold $A$.

$$
\frac{d}{d t} f_{r} \leq A \quad \forall C_{\text {main }}
$$

The coordination of the reliability requirements prescribes the potential energy balance responsible for the bounded deterioration of the system, in other words the normal ageing.

In addition to the degradation of $f_{r}$, the system reliability is conditioned by the performance of the structural support ( $f_{p}$ type II) and mitigation ( $f_{p}$ type IV) functions. This is in contrast to the power transformation and control functions, the failure of which has a direct effect on the system performance. 
The long-term reliability $R_{\text {long }}$ is defined by the distribution of structural loads (type II), as the stresses derived from structural loads are usually the main criteria for the expected lifetime of main components.

$$
\mathrm{R}_{l o n g}=\prod_{C^{I I}} f_{p}
$$

As an example consider a shaft with geometrical deviations from the performance requirement e.g., circular shape. Such abnormal shape leads to a faster degradation of the moving components. This type of deviation in the performance requirement $\theta\left(f_{p}\right)$ is different from a material failure $\theta\left(f_{r}\right)$, such as fatigue cracks. Yet both cases $\theta\left(f_{p}\right)$ and $\theta\left(f_{p}\right)$ accelerate the degradation of the system configuration as a whole.

The short-term reliability $R_{\text {short }}$ is attributed to the mitigation function type. This function type limits the excesses associated with the power transformation functions, such as friction, heat, wear off particles and vibrations. Thus, the failure to mitigate any of these 'residuals' has direct consequences for the system deterioration rate.

$$
\mathrm{R}_{\text {short }}=\prod_{C^{I V}} f_{p}
$$

Thus, the overall system reliability depends on the coordination of the different reliability and performance parameters.

$$
\text { Reliability } \approx \prod_{C} \frac{d}{d t} f_{r} \times \prod_{C^{I I}} f_{p} \times \prod_{C^{I V}} f_{p}
$$

Two preliminary conclusions are drawn from the previous derivations:

- systems are designed to deliver the most efficient energy transformation between the input and output state and to display the lowest degradation possible, and

- to a certain extent, system performance and system reliability depend on distinct functional parameters

The last conclusion can be paraphrased as: similar to the performance and reliability dependencies identified at the component level ( 2.2), also at the system level it is possible to identify a designed (degree of) dependency between system performance and system reliability. Similarly to 2.3 , system level performance and reliability can be represented as: 
where the product symbol refers to the designed dependency. Figure 2.9 illustrates the system dependencies according to Equations 2.10 and 2.18.

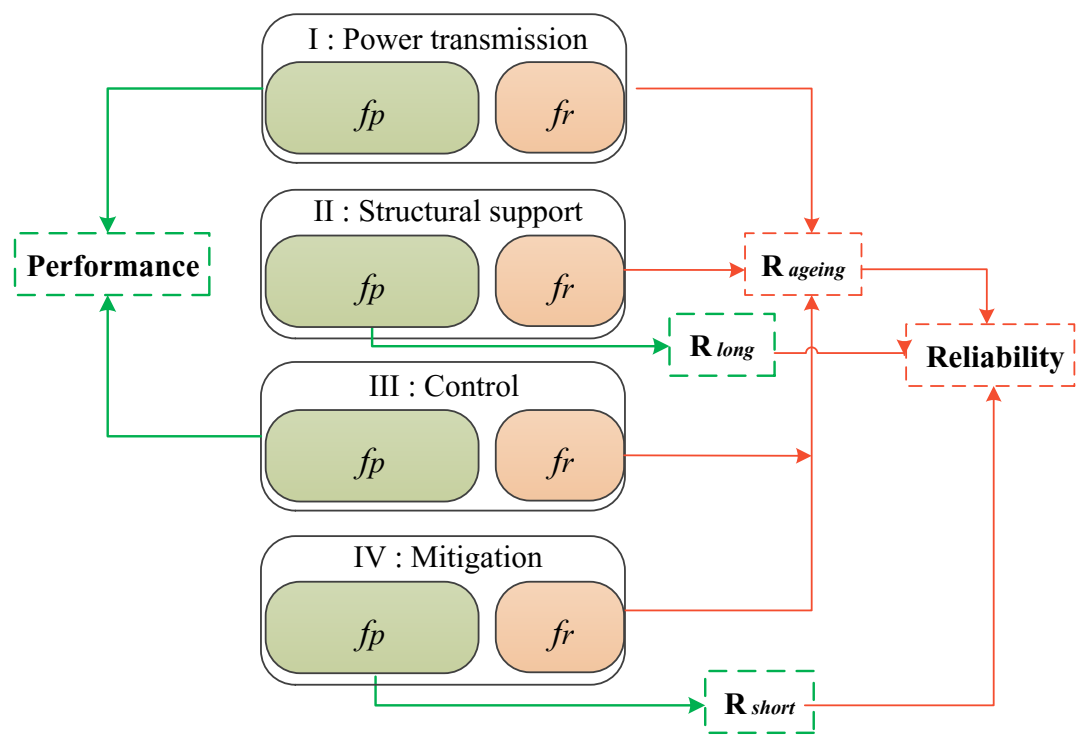

Figure 2.9: Designed, system level performance and reliability dependency.

\subsection{Failure states}

Based on the definition of complex failure, failure will be treated as transformation of the system load distribution, for which new failure states $T_{i}$ emerge replacing the designed state $T_{0}$.

$$
\Theta=\left\{\frac{T_{i}}{T_{0}}\right\}
$$

Note that whereas $T$ has been defined as the state transformation of the resource external to the system (Eq. 2.4), $\Theta$ represents a temporal transformation of the system itself. 


\subsubsection{Effect on performance and reliability}

The effect of failure transformation on the system performance and reliability is discussed next. In comparison with the designed state (Eqs. 2.7 and 2.8), systems undergoing complex failures yield to lower efficiency in the power transformation function. Thus:

$$
\text { Performance }\left(T_{i}\right)<\text { Performance }\left(T_{0}\right)
$$

Also in comparison with the reliability for the designed case (Eq. 2.15), the presence of failure modes accelerates the deterioration rate of a subset $C^{\prime}$ of the system components to levels higher than the designed threshold $A$.

$$
\frac{d}{d t} \theta\left(f_{r}\right)>A \quad \forall C^{\prime}
$$

\subsubsection{Composition of failure transformation}

The composition of the transformation $\Theta$ is addressed next. It will be argued that the system-level transformation is comprised of the aggregation of smaller transformations i.e. failure modes $\theta$.

$$
\Theta=\sum_{C^{\prime}} \theta
$$

Where the aggregation of failure modes by a sum symbol $\sum$.

It will be argued that the addition of a each new failure mode does not necessarily leads to a new failure state. Also, that the combined effect of multiple failure modes is not necessarily the same as the individual effects. Instead, it is possible that new failure behaviours emerge from the synergy of the participating failure modes. More about the additive character of failure modes will be discussed in Section 3.4 and Chapter 6.

By combining equations 2.3, 2.20 and 2.23, the failing state is defined as

$$
T_{i}=\sum_{C^{\prime}} \theta\left(\prod_{C} f\right)
$$

for a simple case where the $\Theta$ is defined by one failure mode $\theta_{C^{\prime}}$ (i.e. $i=1$ ) this is rewritten as 


$$
T_{i}=\theta_{C^{\prime}}\left(\prod_{C} f\right)
$$

which can be further split between the subset of components affected by failure $C^{\prime}$ and the complementary subset $C \backslash C^{\prime}$

$$
T_{i}=\theta_{C^{\prime}}(f) \times \prod_{C \backslash C^{\prime}} f
$$

The effect of failure modes $\theta$ on $f_{p}$ and $f_{r}$, is analysed next. According to Tinga [42] a failure mode is the 'manner in which a system or component functionally fails, that is, describing to what extent a certain function cannot be fulfilled any more'. Based on this definition, it could be argued that failure modes affect principally $f_{p}$ as the functions refer mainly to the system performance. Also the condition that the functions cannot be fulfilled any more is interpreted as the affected $f_{p}$ entering a nonlinear regime as identified by the failure-induced nonlinearities (Sec 2.2.2).

Based on these considerations two types of failure modes, passive and active, are identified. Active failure modes involve changes in the system loads affecting $f_{p}$ directly. Passive modes affect the material capacity $f_{r}$ and depending on the type of dependency $f_{p}$ as well. In both cases $\theta$ changes the designed relation between functional and material degradation, leading to failure-induced interdependencies $(*)$ as

$$
\theta(f)=f_{p} * f_{r}
$$

where $(*)$ denotes failure-induced dependency Finally, by combining Equations 2.25 with 2.3 and 2.26 the failure state is described as

$$
T_{i}=\left(f_{p} * f_{r}\right) \times \prod_{C \backslash C^{\prime}}\left(f_{p} \times f_{r}\right)
$$

Using the example in Figure 2.8 Equation 2.28 can be interpreted as shown in Figure 2.10. 


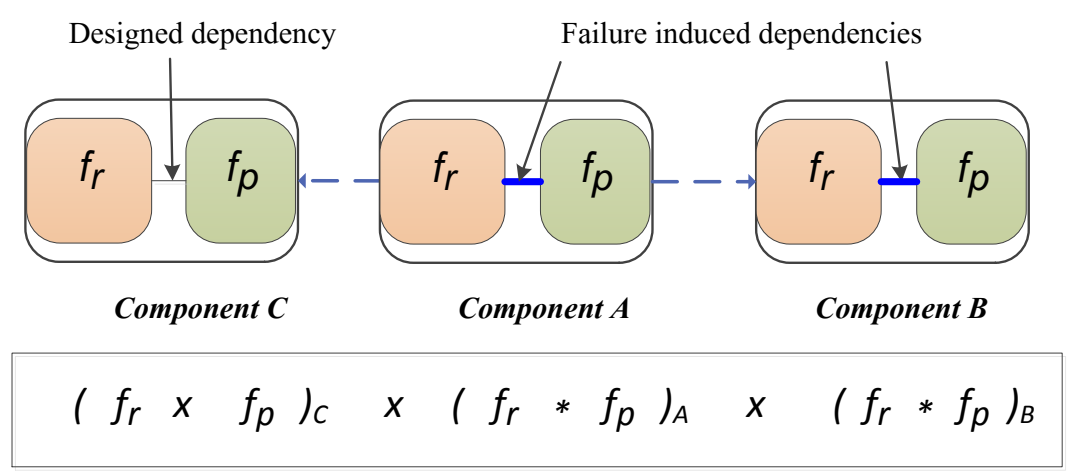

Figure 2.10: Example of failure state

From Equation 2.28 it is inferred that the greater the number of components displaying failure-induced dependency, the greater the deviation from the designed state. Consequently there is an overall change in the system level performance and reliability dependency. Thus, Equation 2.19 is rewritten for the failing system as

$$
\text { Performance } * \text { Reliability for } T_{i}
$$

\subsection{Conclusions}

This chapter identified the change of the dependency relation between performance and reliability parameters at the component level as the main mechanism of complex failure. While there are several dependency types that increase the designed complexity of rotating mechanical systems, these do not necessarily lead to abnormal degradation if estimated correctly in design. Instead, the increased dependency between material and functional degradation leads to operation under nonlinear regimes. Consequently, the system experiences a reorganisation of the load distribution characteristic of complex failure.

The increased understanding of failure-induced dependencies can lead to better estimation of the failure proliferation beyond the component level. Such estimation requires the identification of the functional relations between different system components. In the case of rotating mechanical systems it was shown that these exhibit a particular functional organisation, with distinctive hierarchy and dependencies according to their main function with respect to power transference. 
Understanding the mechanism of complex failure leads to better opportunities to interpret changes in the system behaviour. This means that whenever a system behaves in accordance with new emerging, nonlinear patterns it is possible to infer that the system is entering a new operational, failure-induced state. The dynamics of complex failure behaviour, i.e. how systems behave as they transit through different failure states, is discussed in the next chapter. 


\section{Chapter 3}

\section{Dynamics of complex failure}

Unlike normal ageing, complex failure entails a continuous change the system load distribution. As discussed in the previous chapter, the load distribution changes occur due to the proliferation of increased or exacerbated dependencies between the material degradation and the functional deterioration throughout system components. In consequence of the failure-induced dependencies the system undergoes a reduced linearity on the interactions between components. This implies that as systems are not designed to operate with failure-induced nonlinearities, the emerging behaviour cannot be predicted using the design models.

This chapter addresses the dynamic character of the changes in the system behaviour from an emerging complexity perspective, as introduced in Section 1.5.2. This aims to predict the changes in the system behaviour by understanding the driving principles that govern the multiple nonlinear interactions of its components. In the context of failure of rotating mechanical systems, these principles relate to the diverging and progressive characteristics of failure. Based on the previous discussion, the second research question is reformulated as:

2. What are the principles that govern the diverging and progressive nature of failure behaviour for rotating mechanical systems?

This chapter is organised as follows. Section 3.1 elaborates further on the concept of failure behaviour and failure states. Section 3.2 discusses the divergence of failure behaviour. The progressive nature of failure is addressed in Sections 3.3 and 3.4, which describe the variations within and between failure states respectively. A summary is presented in Section 3.5. 


\subsection{Failure behaviour}

As introduced in Section 1.5, failure changes the structure (material degradation) and function (unfulfilled goals), and behaviour (relation between components) of systems. The pattern of the changes on system behaviour as a consequence of failure is known as failure behaviour.

Figure 3.1 illustrates the designed system behaviour and its changes due to failure. The designed state $T_{0}$ is represented by the black line, where the maximum performance efficiency (Eq. 2.8) is represented by the vertical position. Small variations from the vertical position - grey area - are allowed as a response to changes at the input or output states $\Delta S$, yet the system is expected to return to the highest operational setting by means of the control functions (type III).

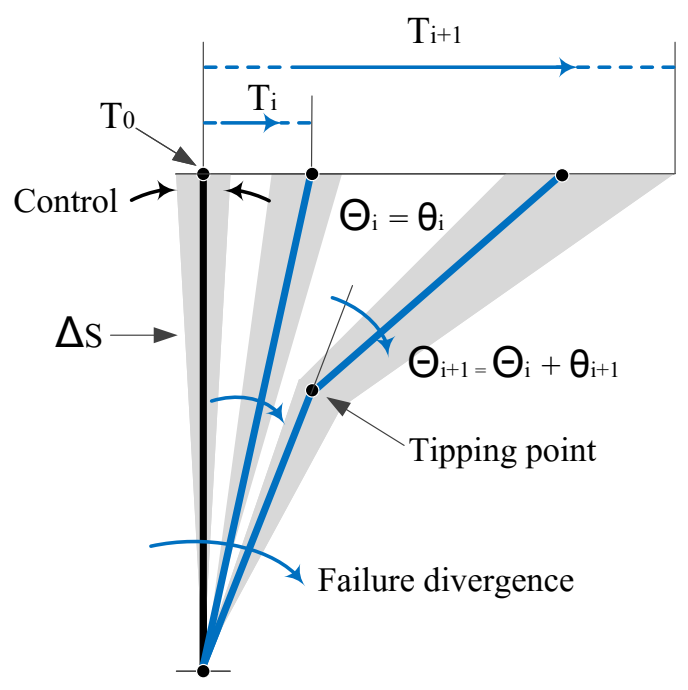

Figure 3.1: Divergence of failure states.

Figure 3.1 also represents subsequent failure transformations $\Theta_{i}$ and $\Theta_{i+1}$, leading to the failure states $T_{i}$ and $T_{i+1}$ respectively. Failure modes are illustrated as perturbation of the system behaviour, as each failure mode has characteristic origins (material degradation) and effect (functional degradation). The first transformation of the $\Theta_{i}$ occurs due to the failure mode $\theta_{i}$. The changes in the system behaviour continue until a tipping point is reached when a second failure mode $\theta_{i+1}$ appears. 
Further deviations from the designed behaviour are in line with the transformation $\Theta_{i+1}$, which results from the combined effect of the previous failure state $\Theta_{i}$ and the incoming failure mode $\theta_{i+1}$.

Implicit to the definition of failure states is that the changes in the system behaviour are modest for within-state and abrupt for between-states. Yet there is always a tendency for further divergence of the system behaviour away from the designed state, as discussed next.

\subsection{Divergence}

Failure divergence refers to the continuous deviation away from the designed state, which tends to increase over time rather than to decay or remain stable. The increasing divergence is attributed to positive feedback loops that amplify a response. Contrary to negative feedback exerted by control which corrects deviations in the system behaviour towards the designed behaviour, the changes associated with failure alter the system further. For the context of failure of rotating systems, the positive feedback is attributed to the energy that is not transferred effectively as the system's main function, but instead is trapped within the boundaries of the system, feeding further system degradation. This is presented as the loss energy principle:

Loss Energy Principle Failure increases the system entropy by reducing the effectiveness of the functional energy transformations while accelerating the system degradation.

The loss energy principle is consistent with the increased dependency between performance and reliability as derived in the previous chapter (Eq. 2.29). Furthermore, it establishes a proportionality between these: the greater the performance drop, the faster the acceleration of the system degradation.

$$
\text { Performance } \propto \text { Reliability for } T_{i}
$$

As stated in Section 2.3, coordination of performance requirements prescribes the kinetic energy balance of the system, while reliability requirements prescribe the potential energy balance responsible for the bounded deterioration of the system. This means that failure changes in the energy balance of the system and increases its entropy. 


\subsection{Progressive: within-state variations}

The progressive nature of failure derives from the heterogeneous consumption of material capacity under complex failure. Yet, given failure divergence character, the system behaviours change continuously even within the failure states. However, as each failure state is defined by the failure modes that trigger material and functional degradation, the changes within failure states can only be quantitative.

According to the loss energy principle, the kinetic energy that is not effectively transformed as part of the system main function is shifted towards the system itself, accelerating its degradation. This means that system degradation, or the rate of degradation, depends on the amount of kinetic energy involved in the failure transformation. Three distinct sources of kinetic energy are identified.

The first source refers to the functional role of the affected components as proposed through the functional hierarchy in Section 2.3.1. Thus, whereas the power transfer (type I) and the control function (type III) are the most likely to influence the characteristics of the transferred loads, support function (type II) and the mitigation functions (type IV) exhibit a more passive role. Consequently, the effects on the two former types of functions are likely to have a bigger impact on the system degradation than the latter types.

The second source corresponds to the actual physical damage: the likelihood of releasing more wasted energy depends on the type of failure mechanisms involved and their interactions as seen in Section 2.1.1, but also the location and the size of them.

The last source is the external operating environment. As stated before, systems are designed for a preferred input state (Eq. 2.8), yet the design must guarantee system behaviour stability despite moderate variations of the input state. However, such stability cannot be guaranteed once the failure takes place, once it is not possible to predict how failure and the environment interact.

These three sources of kinetic energy are also considered as sources of uncertainty for the failure assessment. Thus, the capacity to estimate the current effect of failure on the system behaviour and to predict its future evolution depends on the knowledge of the current functional and material degradation and how these are affected by the varying operational environment. This is stated in the second principle as:

Kinetic Energy Principle Failure evolution depends on the destabilisation effect of kinetic energy with respect to the compromised functions, the physical damage characteristics and the external environment variability. Consequently, the likelihood of predicting failure evolution is conditioned by the uncertainties associated with each of these factors. 


\subsection{Progressive: between-state variations}

In Section 2.4.2 the concept of failure state was defined as the aggregation of different failure modes taking place simultaneously at the system. As already introduced, the transition to a new failure state implies a qualitative change in the system behaviour due to an additional perturbation of the system, i.e., failure mode. This is described as

$$
T_{i+1}=f\left(T_{i}, \theta_{i+1}\right)
$$

As discussed in Section 2.4.2 each failure mode leads to a different transformation of the system, yet that does not necessarily mean that each failure mode triggers a new failure mode. In general three types of relation between the failure state and the incoming failure mode are identified as shown in figure 3.2.

- Independent

$$
\Theta_{i}+\theta_{i+1} \rightarrow \Theta_{i+1}
$$

- Equivalent

$$
\Theta_{i}+\theta_{i+1} \rightarrow \Theta_{i}
$$

- Magnitude-dependent $\left\{\begin{array}{l}\Theta_{\mathrm{i}}+a \theta_{\mathrm{i}+1} \rightarrow \Theta_{\mathrm{i}} \\ \Theta_{\mathrm{i}}+c \theta_{\mathrm{i}+1} \rightarrow \Theta_{\mathrm{i}+1}\end{array}\right.$ for $a<c$

Figure 3.2: Failure state - failure mode dependency types.

In the independent case the addition of a failure mode leads to a new failure state, similar to the example in figure 3.1. Thus, the perturbation excites new nonlinearities that are different from those already existing at the base failure. For the second type the incoming failure mode does not trigger a new failure state. In this case the perturbation has an equivalent effect to those at the base failure state. The latter type occurs when the new failure state is triggered only when the magnitude of the incoming failure mode is above a certain threshold. In this case there is a magnitude-dependent relation.

In addition, failure modes have a different effect on the system behaviour when occurring over the designed state than over an already existing failure state. This means that systems undergoing abnormal degradation have an increased chance of reaching new failure states when subjected to an external perturbation. The degree that a new failure mode triggers a new failure state is referred to as mode-state dependency. Figure 3.3 presents a visual representation of this concept. 


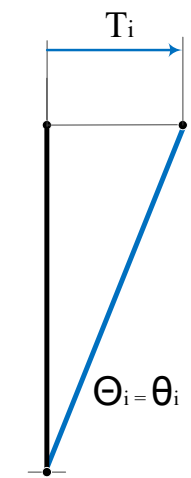

a) Base failure state

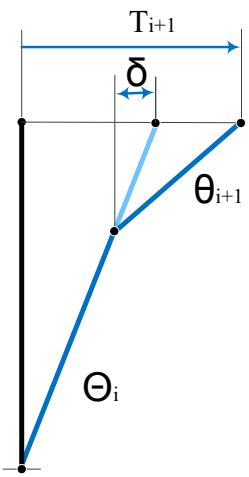

b) low dependency

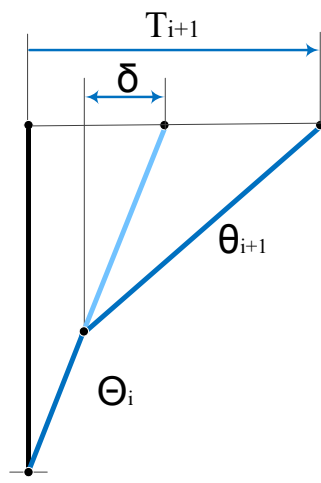

c) high dependency

Figure 3.3: Dependency $\delta$ between failure states $\Theta_{i}$ and incoming failure mode $\theta_{i+1}$

Frame $a$ corresponds to the base failure state $T_{i}$ caused by one initial failure state $\Theta_{i}$. Frames $b$ and $c$ represent two emerging states $T_{i+1}$ as a consequence of the new additional failure mode $\theta_{i+1}$. The dependency between the incoming failure mode and the base failure state is represented by $\delta$. A higher dependency leads to a more significant effect on for the new failure state as shown in frame $c$.

It will be argued that the effect of an incoming failure mode on a failure state (i.e.,, to trigger a new failure state and the resulting changes in the system behaviour) depends on the actual system complexity. Actual system complexity refers to the increased (functional) interdependencies due to the changes in the material degradation and functional deterioration associated with the failure base state as described in Section 2.2.2.

Thus, for the base failure state the system is already under an altered load distribution, which causes an abnormal degradation. For some components this implies a faster consumption of the material capacity and consequently a greater likelihood of entering the nonlinear range between material degradation and functional deterioration (failure-induced dependencies). Therefore the incoming failure mode accelerates further the transition to the nonlinear ranges if it affects the already compromised functional parameters directly or indirectly. This is formulated as the modestate dependency principle:

Mode-State Dependency Principle The likelihood of an incoming failure mode (perturbation) triggering a new failure state increases with the actual system complexity (interdependency). 
This principle emphasizes that new failure states are more likely to emerge in systems with a high degree of inter-dependencies, due to the designed configuration and the accumulated failure modes. However, predicting the effects of an incoming failure mode is limited by the uncertainty (lack of knowledge) about the set of functions compromised at a given failure state. Thus, according to the definition of complexity presented in Chapter 1 failure increases the system complexity as it becomes difficult to understand how the functions become increasingly interdependent through the effect of failure.

\subsection{Summary}

This chapter addressed the principles that allow the prediction of complex failure dynamics. First at all, failure is recognised as a dynamic transformation of the system behaviour, the main characteristics of which are its diverging and progressive nature. Based on these characteristics, three failure principles are proposed:

- Loss Energy Principle: Failure increases the system entropy by reducing the effectiveness in the functional energy transformations while accelerating the system degradation.

- Kinetic Energy Principle: Failure evolution depends on the destabilisation effect of kinetic energy with respect to the compromised functions, the physical damage characteristics and the external environment variability. Consequently the likelihood of predicting failure evolution is conditioned by the uncertainties associated with each of these factors.

- Mode-State Dependency Principle: The likelihood of an incoming failure mode (perturbation) triggering a new failure state increases with the actual system complexity (interdependency).

According to these principles failure is defined as a diverging and progressive transformation of the system behaviour that increases the system entropy, uncertainty and complexity (interdependency) with respect to the designed state. Each of these aspects - entropy, uncertainty and complexity (interdependency) - are required to be addressed on the basis of observations of the failure behaviour. This is discussed in the second part of this thesis. 


\section{Part II}

\section{A Functional Approach to Vibration Analysis}





\section{Chapter 4}

\section{Entropy: Vibration response analysis}

\subsection{Introduction to the second part}

The second part of this thesis addresses the analysis of vibration behaviour as a means of increasing the capacity to observe complex failure and, more specifically, to interpret the vibration response in relation to the changes in the dynamic load distribution.

What does observation actually mean? As discussed in the introduction, the current focus on predicting the remaining useful life given an identified failure mechanism and failure mode is valid only for simple failure. The study of complex failure requires an approach focused on the load distribution at system level. Under this approach the detection, diagnostics and prognostics efforts must address multiple aspects of the mechanisms and dynamics of complex failure. As a guideline, Figure 4.1 presents a list of relevant questions that can help in the assessment of load distribution.

The potential of vibration analysis to observe complex failure lies in the interpretation of the changes in the system load distribution. Yet achieving this potential requires a comprehensive understanding of the elements of the vibration response and how their interactions change as a consequence of failure. The next three chapters discuss the use of vibration behaviour for the observation of failure, specifically in relation to the increased entropy, uncertainty and complexity. 


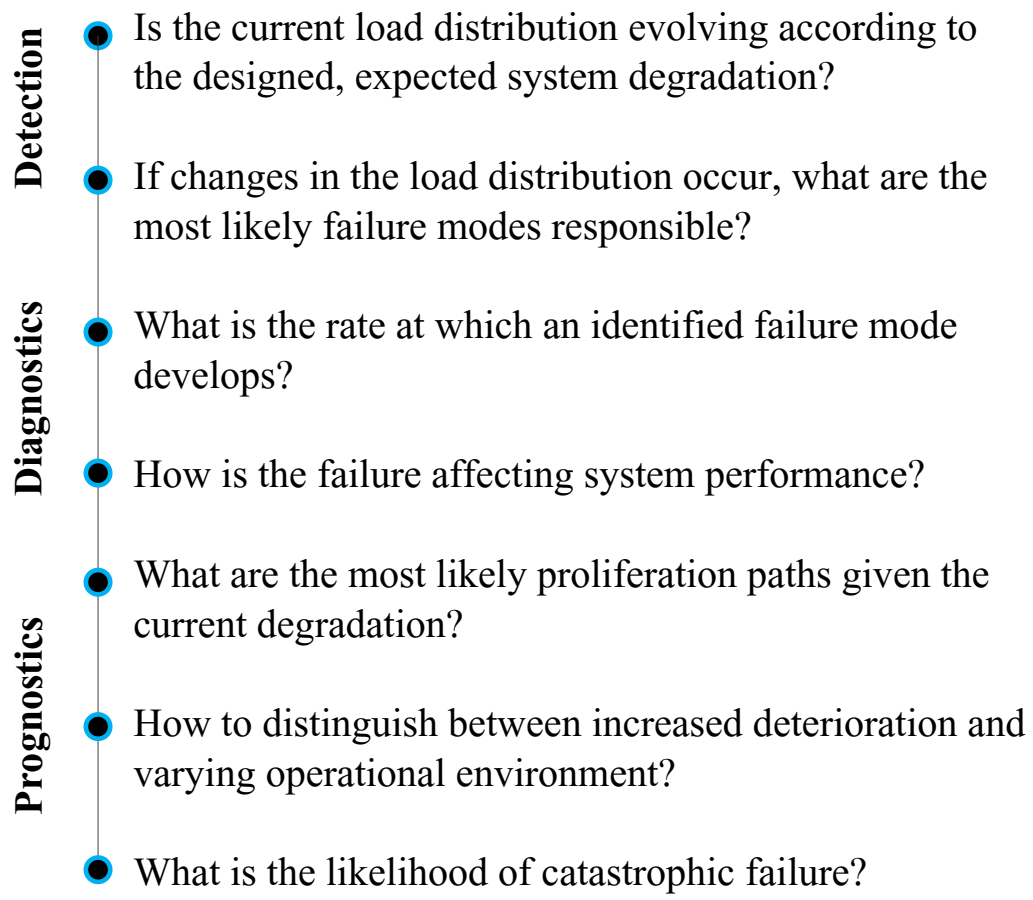

Figure 4.1: Guiding questions for comprehensive failure assessment.

\subsection{Vibration as loss energy}

As seen in Chapter 2, failure changes the system configuration that guarantees the maximum efficiency in the power transformation function. The energy that is not transmitted effectively becomes trapped within the boundaries of the system, feeding further degradation. This was stated in the loss energy principle as:

Loss Energy Principle Failure increases the system entropy by reducing the effectiveness of the functional energy transformations while accelerating the system degradation.

The loss energy principle enables a functional approach to vibration analysis. As the primary function of rotating mechanical systems is to transform energy, changes in the energy balance can be related to the system performance and reliability. 
Currently, vibration analysis is addressed from two different perspectives. The industrial practice for which operating systems are monitored to warn against increasing vibration levels. And the academic developments, focused on the accurate detection (i.e. signal processing) and prognostics (i.e. machine learning) based on the changes in the vibration pattern. Although these perspectives seem complementary, in fact they often develop independently of each other. This is stated by Sikorska [35]: 'RED tends to be driven by the desire to fit a model to an application while practical implementation is driven by the tools (people, software programs etc.) available.'

It has already been argued in the introductory chapter that the lack of integration between the different approaches to failure, or the study of failure, is due to the limited understanding of the failure phenomenon at the system level. Yet, for the specific case of vibration monitoring, this chapter argues that reconciling the academic developments and the industrial practice requires a consensus on the meaning of the features derived from the vibration signal and specifically on the requirements on the feature selection to characterise load distribution changes. This was presented as the third research question of the thesis:

3. What are the features of the vibration response that enable the interpretation of load distribution changes associated with complex failure?

This chapter demonstrates that vibration behaviour is a consequence of the increased entropy of the system. Additionally, a functional approach to the requirements selection for vibration features to characterise changes in the load distribution is presented. The chapter is organised as follows: Section 4.3 introduces basic definitions regarding the vibration response. Section 4.4 discusses the nonlinear interactions on the vibration response. Section 4.5 discusses the role of the identified elements for the assessment of the system functionality and load distribution. Conclusions are presented in Section 4.6.

\subsection{Elements of vibration response}

Maintenance is a topic not typically associated with innovation. As stated by Prof. Tinga, 'technical universities and colleges mainly train their students to design new machines and systems, i.e. so to be innovative. However, once graduated, the vast majority of them will mainly be busy keeping existing machines and systems running. ${ }^{1}$ This statement reflects how, besides the study of material degradation, the topic of failure receives in general less attention from a physics perspective in comparison with the topic of design.

\footnotetext{
${ }^{1}$ Translated from the article: He innovators, gamechangers en disrupters, vergeten jullie het onderhoud niet! Source decorrespondent.nl
} 
A failure focus different from the design focus recalled in the statement of Prof. Tinga is necessary in order to understand the nature of abnormal vibration behaviour and, in consequence, to support the observation of failure. Such focus must address the nature of the failure-induced vibration behaviour, originating from forces that do not contribute to system torque and unwanted system resonances. This is different from the designed dynamic behaviour, which derives from the operational forces that maximise system performance while restricting system resonances. The following sections set out the basic definitions of vibration behaviour assessment.

\subsubsection{Basic definitions}

In a general sense, mechanical vibrations are the oscillatory response of the system to a dynamic force, as explained in Figure 4.2. This definition emphasises the dynamic forces, the system and the response as main elements of the vibration phenomenon.

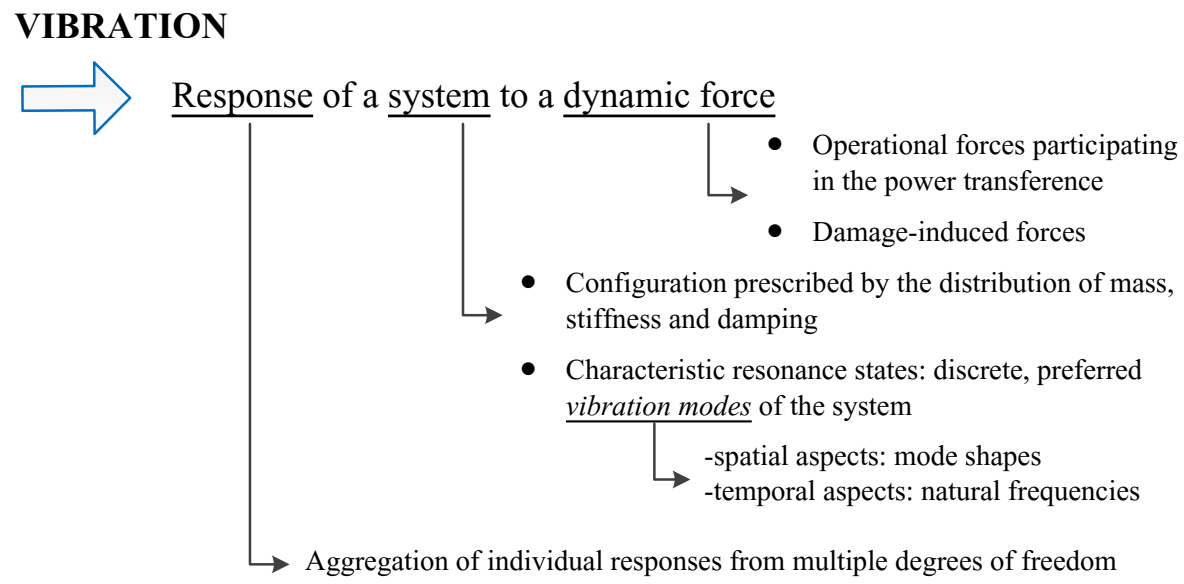

Figure 4.2: Elements of the vibration phenomenon.

As introduced in Chapter 2, the system refers to the set of components whose interactions enable a power transformation between an input and output state. When circumscribed to the dynamics aspects, the system configuration corresponds to the distribution of mass, stiffness and damping that define the components' interactions. Such distribution leads to the distinctive dynamic behaviour of the system, expressed by the resonances or intrinsic vibration modes. 
Resonances are discrete states where the incoming energy from the input state is diverted to the system itself instead of being transferred. The most characteristic aspect of resonances is the large internal deformations that must be avoided for the stable operation of the system. The spatial aspects of resonances are characterised by the mode shapes or eigen-modes, whereas the temporal aspects are characterised by the natural frequencies or eigen-frequencies. Resonances can affect the system on a global scale as the corresponding mode shapes extend throughout the system, or on a local scale when their effect is limited to sub-sections or sub-assemblies of the system.

The dynamic forces exciting the system resonances originate either from the power transformation or from (local) damage. The direction of these forces indicate whether they contribute to the system function or rather amplify the vibration behaviour of the system. Figure 4.3a illustrates a rotating shaft with eight blades that enable the power exchange between the surrounding environment and the shaft. The power exchange involves the force $F_{o p}$ at each blade, which comprises a perpendicular component $F_{Q}$ contributing to the shaft torque $Q$, and a lateral component $F_{X}$ reflecting as as lateral vibration $X$. Hereafter the variable $F_{o p}$ will be used to indicate the operational origins of the vibration response.

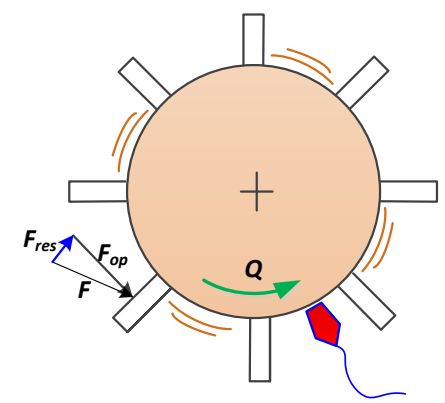

(a) Rotating shaft.

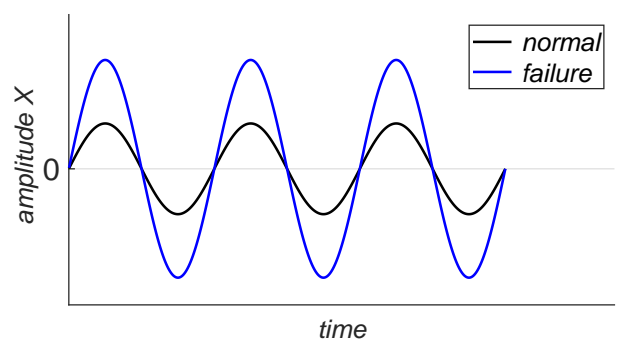

(b) Vibration response.

Figure 4.3: Lateral vibration due to residual forces from the power transformation.

It must be noted that even for the designed state, mechanical systems display lateral vibrations due to the varying compliance of the transmission elements [65-67] and residual geometrical asymmetry around the rotation centre. However, systems are designed for limited residual forces that do not disrupt the overall load distribution. 
The response corresponds to the aggregation of multiple individual responses to forces and resonance combinations. The operational forces are reflected only in the torsional vibration of the system. Instead, as mentioned before, the residual forces are reflected in the lateral vibrations.

Figure 4.3 helps to explain the relation between the loss energy principle and vibration behaviour. It is argued that the total force $F$ has a limited magnitude (potential) set by the characteristics of the input state (e.g. wind speed, flow rate). As discussed in Section 2.3, the designed configuration is chosen so as to maximise system torque (Equations 2.8) and to guarantee a bounded degradation of the main components (Equation 2.12).

Furthermore, this section argues that the residual forces are limited, so they do not interfere with the power transformation function. However, failure increases the magnitude of the residual forces, either due to changes in the operational loads (e.g. arising from complicated failure or complex failure) or due to local damage (e.g. arising from simple failure).

These considerations suggest that any significant deviation from the designed $F_{Q}-F_{X}$ relation implies the decrease in the system performance and the accelerated system degradation as stated in the loss energy principle, while increasing the lateral vibrations of the system. This justifies the use of lateral vibration response as a direct estimator of the entropic forces, which do not contribute to the generated work of the system and which destabilise the load distribution of the system instead.

\subsubsection{Signal decomposition}

The core task of the signal treatment is to decompose the vibration response into meaningful features that reflect the effect of specific forces on critical modes of the system. Similarly to the system resonances, the vibration response can be decomposed according to the spatial and temporal aspects, and in line with structural and rotating perspectives shown in Table 4.1.

Table 4.1: Decomposition of the vibration response from structural dynamics and rotating dynamics.

\begin{tabular}{|c|c|}
\hline $\begin{array}{l}\text { Structural dynamics } \\
\text { - } X: \text { : the total response } \\
\text { - } \quad \hat{x_{i}}: \text { the normalized mode shape } \\
\text { - } \quad a_{i}: \text { participation factor }\end{array}$ & $\begin{array}{l}\text { Rotating dynamics } \\
\text { - } \quad X(i \omega) \text { is the total response } \\
\text { - } \quad \hat{x}(i \omega) \text { is a constituent from the } \\
\text { frequency spectrum }\end{array}$ \\
\hline
\end{tabular}


In sum, the structural dynamics perspective estimates the participation factors of the distinct mode shapes in the total system response. Instead, the rotating dynamics perspective breaks down the signal in relation to the frequency content of the driving forces and the excited eigen-frequencies. The latter is the most frequently used approach to the diagnostics of rotating mechanical systems, thus it is the focus of the rest of this chapter.

The treatment of the vibration response is composed of two phases. The first phase corresponds to the decomposition of the vibration signal. As shown in Table 4.1 this can be expressed as

$$
X(\omega)=\sum \chi(\omega)
$$

where $X(\omega)$ represents the lateral vibration response and $\chi(\omega)$ the individual constituents. The second phase corresponds to the identification of the forces and resonances participating in the vibration response. Thus,

$$
\chi(\omega)=F_{i}(\omega) \cdot H_{i}(\omega)
$$

where $F_{i}(\omega)$ corresponds to the individual lateral (residual) force and $H_{i}(\omega)$ to the resonance excited.

The form of the Equation 4.2 corresponds to a transfer function formulation, for which $H_{i}(\omega)$ is the frequency response function containing information about the system dynamic properties. This is illustrated in Figure 4.4.

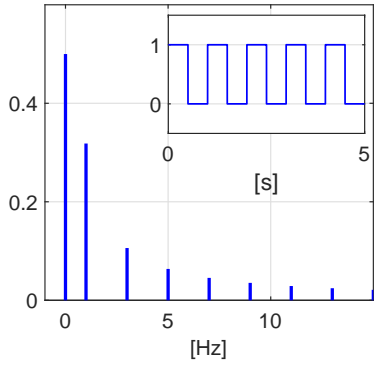

(a) $F_{i}(\omega)$

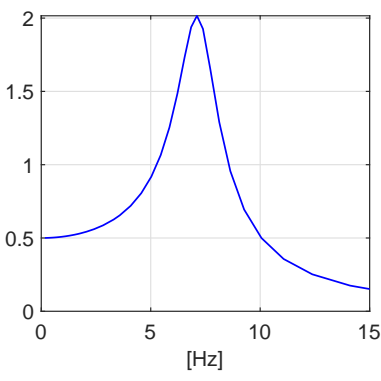

(b) $H_{i}(\omega)$

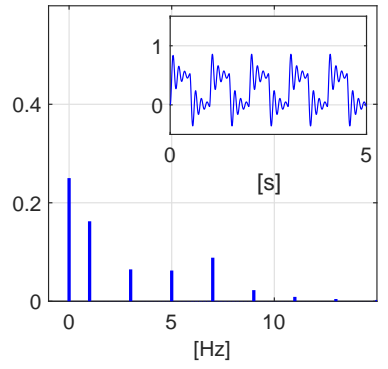

(c) $\chi(\omega)$

Figure 4.4: Example of Frequency Response Function. ${ }^{2}$

\footnotetext{
${ }^{2}$ Modified from Frequency Response Model in Wikipedia. CC BY-SA 3.0.
} 
The main types of forces and modes are classified as:

Operational forces $\left(F_{o p}\right)$ The residual, lateral forces resulting from the power transformation of the system. These can relate directly to the individual power transmission elements (i.e. blades, gears, pulley) or to the imbalance forces at the shaft.

Damage-induced forces $\left(F_{d}\right)$ These refer to internal forces originating from increased non-conservative forces (e.g. friction) or from the independent motion of local sub-assemblies.

Main structural resonances $\left(H_{s t}\right)$ These result from the overall distribution of mass, stiffness and damping in the system.

Damage-induced resonances $\left(H_{d}\right)$ These refer to the local resonances excited by damage, or structural resonances from sub-assemblies that become loose.

The decomposition of the vibration response involves significant challenges given the nature of rotating mechanical systems. Firstly, the multiplicity of operational forces that convolute in a mechanical system - as in the case of gearboxes, where each gear pairing involves a force exchange with distinctive direction, magnitude and frequency. Also different forces and resonances can occur at close frequencies making it difficult to distinguish them. Finally the variation in the operational environment can influence the characteristics (i.e. frequency content, mode shape) of the forces and resonances.

\subsection{Non-linear interactions}

As stated in Section 2.2.1, mechanical systems are designed to operate within linear ranges. This linearity implies that the transfer function describing the designed system is invariant for the operating ranges of the input and output states (Equation 2.4). The system linearity is also reflected in the vibration behaviour as the multiple lateral forces coexist, yet their limited magnitude guarantees that they are independent of each other. For the gearbox example this means that each contact between teeth involves a stable force, not dependent on any other contact pair or on the residual imbalance forces at the shaft. Thus, in the absence of failure, variations in the vibration behaviour respond only to the operational environment.

As discussed in Section 2.4, failure increases nonlinear relations between system components. The vibration response also reflects the transition to nonlinear regimes as changes in the signal composition, both at the individual vibration responses and for the interaction between them. The nonlinear interactions most common to rotating mechanical systems are discussed next. 


\subsubsection{Harmonic distortion}

The increase of residual forces leads to higher displacements of the rotation shaft (Figure 4.3b) up to the point where the physical limits of the system (i.e. shaft housing, clearances) limit further amplitude growth. From this point onwards, the shaft orbit adopts new shapes to accommodate to the increased loading, for instance by truncating the signal. Such changes are referred to as harmonic distortion since the spectral representation of the distorted signal requires a higher number of harmonics (multiple of the fundamental frequency) to represent the new shapes.

An example of harmonic distortion due to truncation is shown in Figure 4.5. The time waveform (Figure 4.5a) shows a comparison between a reference sinusoidal, linear signal (black) and distorted one (blue). The spectral representation of the truncated signal is shown in Figure 4.5b. While for the linear signal the spectral representation requires only one frequency component, for the distorted one two additional frequency components are necessary to curtail the extreme acceleration to the limit value (i.e. $1 \mathrm{~m} / \mathrm{s}^{2}$ ).

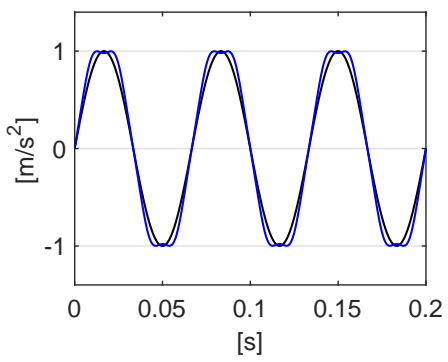

(a) time waveform

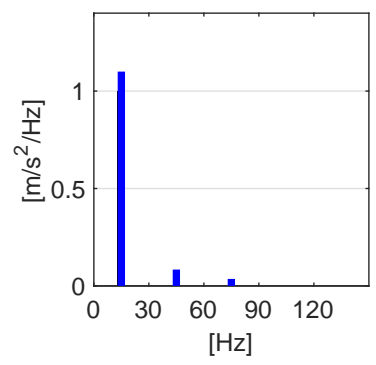

(b) spectrum

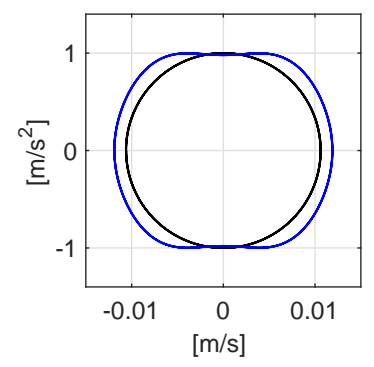

(c) phase portrait

Figure 4.5: Example of Harmonic Distortion.

The phase portrait (Figure 4.5c) provides a representation of the state space (i.e. velocity and acceleration) for the signals. In general, for a perfectly sinusoidal signal the phase portrait shows a circular shape. For the analysed case, the disrupted signals shows a greater amplitude both for the velocity and acceleration dimensions, yet it is curtailed for the acceleration for values above the linear limit.

In general, harmonic distortions are very frequent nonlinear phenomenon associated with failing rotating systems. Failure modes such as imbalance and misalignment are recognised mainly via increased harmonics of the fundamental rotation speed $[68,69]$. Abnormalities at the power transmission elements such as gears and blades show also as increased harmonics of the frequency of the contact forces (i.e. the gear mesh frequency and blade pass frequency) and their harmonics. 


\subsubsection{Frequency modulation}

Frequency modulation is an other source of changes of the shape of the individual vibration response. Yet unlike the amplitude-led distortion discussed before, frequency modulation occurs due to uneven rotational speed. This is explained with the example of lateral vibrations shown in Figure 4.3. The fluctuations in the residual forces at one transmission element lead to variations in the instantaneous torque and angular speed given the conservation of angular momentum. The resulting lateral vibration behaviour exhibits frequency modulation, also known as torsional vibrations.

Figure 4.6 illustrates frequency modulation response. The time waveform (Fig. 4.6a shows the effect of the uneven rotation on the shape of the sinusoidal signal (blue). In comparison to the reference signal (black), the affected rotation is slowed down for some segments while for others is accelerated.

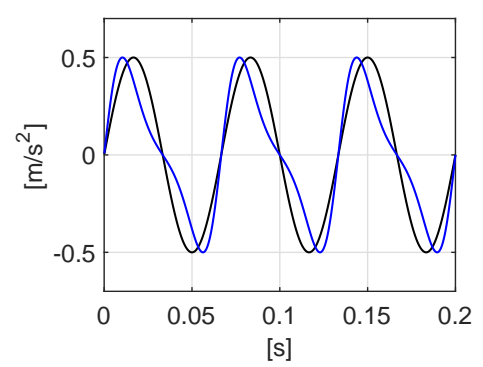

(a) time waveform

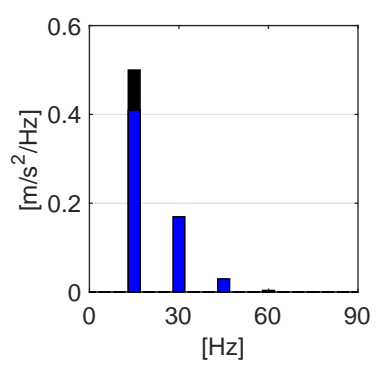

(b) spectrum

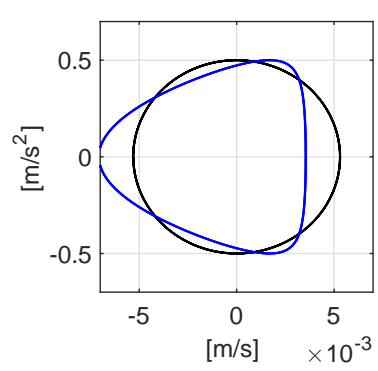

(c) phase portrait

Figure 4.6: Example of Frequency Modulation.

Similarly to the harmonic distortion case, the spectral representation (Fig. $4.6 \mathrm{~b}$ uses additional harmonic components to represent the skewed shape. However this representation can be misleading as it does capture the variations in the angular speed directly. A more reliable representation is shown at the phase portrait (Fig. 4.6c, for which the uneven rotation shows as a skewed, deformed ellipse along the velocity axis. The use of phase portrait will be discussed further in Chapter 6

Frequency modulation occurs due to the uneven contribution of the transmission elements to the overall system torque [70-72]. However, the presence of torsional vibrations is often under reported using the lateral response. Direct shaft measurements of speed and torque are more suitable to the diagnostics of frequency modulation, however these are less common in industrial monitoring systems. 


\subsubsection{Amplitude modulation}

Failure increases the dependency between different residual forces. In the example of the gearbox, the gear pairing and shaft rotation reveal at different frequencies, being the gear mesh frequency the number of teeth in contact times the shaft rotation as shown in Figure 4.7. In this designed case, the magnitude of the residual forces (f.i. due to imbalance) and the gear contact forces are independently of each other. However, if either of these forces increases due to damage, the other is affected as well. This causes a shared interaction where the amplitude of the higher carrier frequency event is modulated by the lower frequency event as shown in Figure 4.8.

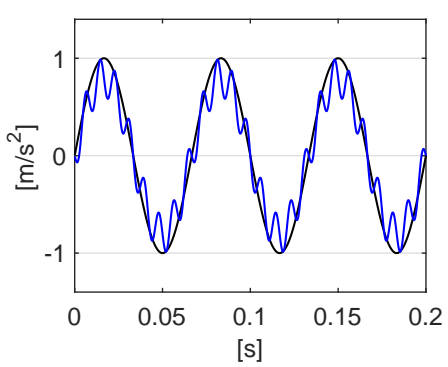

(a) time waveform

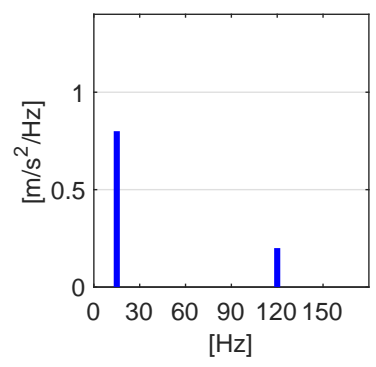

(b) spectrum

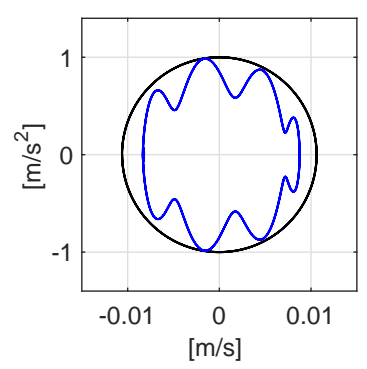

(c) phase portrait

Figure 4.7: Example of independent vibration responses.

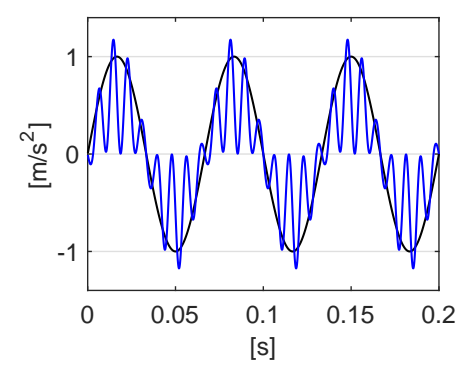

(a) time waveform

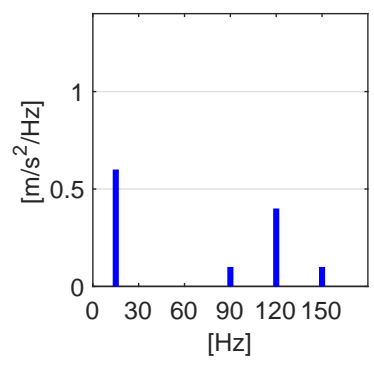

(b) spectrum

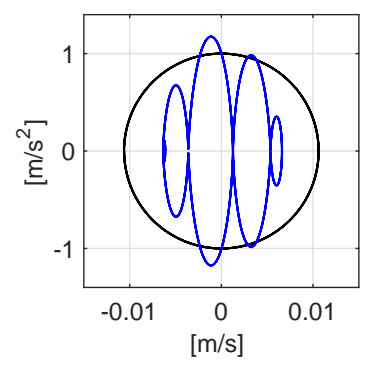

(c) phase portrait

Figure 4.8: Example of Amplitude Modulation.

Note that the phase portrait of the independent interaction shows as a oscillating line on top of the reference circle. Yet, for the modulation case, the oscillation becomes more pronounced and leads to internal buckles. This corresponds to an increase in the system dimensionality (i.e. a higher number of crossing on the abscissa). 
A typical example of amplitude modulation is bearing damage, for which surface damage excites locally induced resonances of the rolling elements. These excitations manifest themselves as impulsive events modulated by the shaft rotation. More about amplitude modulation in roller bearings will be discussed in Chapter 5 .

\subsubsection{Resonance state}

The final type of nonlinear interaction is resonance. As discussed, the system configuration must avoid the correspondence of operational forces $F_{o} p$ and structural resonances $H_{s t}$ to limit the occurrence of a resonance state. From an energy perspective, a resonance state occurs when energy is transferred repeatedly from kinetic to potential form and vice versa. This means that at resonance the kinetic energy drives the motion of individual sub-assemblies, rather than being effectively transferred.

Full correspondence between the operational forces' frequencies and the natural frequencies is uncommon as systems are designed to avoid resonance. However, resonance states can develop progressively due to accumulation of damage or can appear suddenly due to external events, as in the case of looseness. The involved displacements and deformations derived from system resonance are reflected as strong harmonic disruption of the operational forces, as shown in Figure 4.9. In comparison with the clipping behaviour shown in Figure 4.5, during resonance the increased deformations lead to an stronger and more erratic disruption of the vibration response.

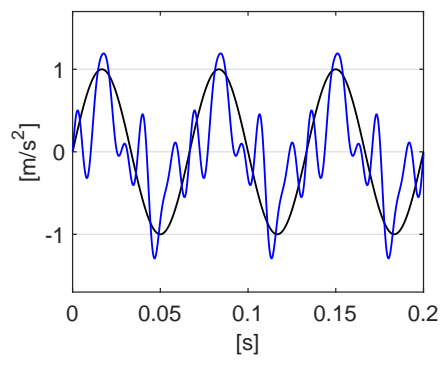

(a) time waveform

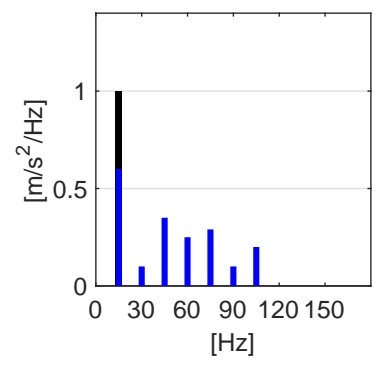

(b) spectrum

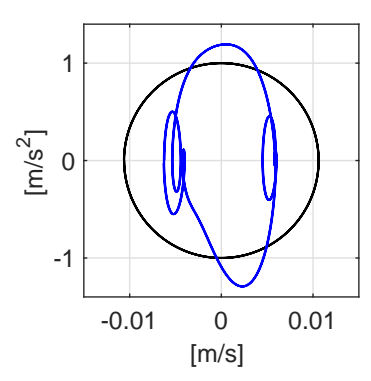

(c) phase portrait

Figure 4.9: Example of strong harmonic distortion typical of resonance. 


\subsection{Failure assessment}

The previous section demonstrated the relation between the lateral vibration behaviour with the destabilisation forces of the load distribution of the system associated with complex failure. This section introduces a general guideline for the evaluation of the effects of failure on the system functionality based on the interpretation of the vibration response.

\subsubsection{Functionality assessment}

Following with the description of system functionality and failure presented in Chapter 2 , this section presents a description of the dynamic aspects of the system functionality $T^{d}$ as a function of elements of the vibration response. Thus, the system functionality can be described from a dynamics perspective as

$$
T^{d}=f(F, H)
$$

where the designed system functionality $T_{0}^{d}$ can be described by a function of the operational forces $\left(F_{o p}\right)$ and main structural resonances $\left(H_{s t}\right)$.

$$
T_{0}^{d}=f\left(F_{o p}, H_{s t}\right)
$$

Consequently, the functionality of a system at a specific failure state $\left(T_{i}^{d}\right)$ can be described by a function of the modified operational forces $\left(\Delta F_{o p}\right)$, the change in main structural resonances $\left(\Delta H_{s t}\right)$, the damage-induced forces $\left(F_{d}\right)$ and the damageinduced resonances $\left(H_{d}\right)$.

$$
T_{i}^{d}=f\left(\Delta F_{o p}, \Delta H_{s t}, F_{d}, H_{d}\right)
$$

The dynamic aspects of the failure transformation (Eq. 2.20 and 2.23) are represented as

$$
\begin{gathered}
T_{i}^{d}=\Theta_{i}^{d}\left(T_{0}\right) \\
\Theta^{d}=\sum_{C^{\prime}} \theta^{d}
\end{gathered}
$$

This means that the degradation of the system functions can be approximated by the changes in the elements of the vibration signal: 
- Failure of the functions related to the power transmission $\left(f_{p}\right.$ type I from hierarchy in Section 2.3.1) are assessed by changes in the operational forces $\left(\Delta F_{o p}\right)$

- Failure of the structural support function ( $f_{p}$ type II) are evaluated by changes in the structural resonances, both for the variations in the main resonances $\left(\Delta H_{s t}\right)$ and the damage-induced resonances $\left(H_{d}\right)$, as modifications in the structural configuration restrain the dynamic forces in a different way than designed.

- Failure of the reliability functions $f_{r}$ are evaluated by damage-induced forces $\left(F_{d}\right)$ and resonances $\left(H_{d}\right)$ as failure increases the non-conservative forces due to accelerated material degradation.

Based on the definition of system performance and reliability presented in Equation 2.10 and Equation 2.18, a classification of the elements of the vibration response with respect to their influence (significance) for system performance and reliability is presented as follows:

- Performance : $\Delta F_{o p}$ as it relates directly to the energy of the power transformation.

- Reliability : $\Delta H_{s t}, F_{d}$ and $H_{d}$ for $T_{i}^{d}$ as any altered configuration and damageinduced forces leads to inhomogeneous and accelerating degradation of system components.

The presented classification is used to monitor the changes in the system entropy. On the one hand, the reduction in effective operational forces (contributing to the system torque) implies a lower system performance. And on the other hand, as the main structural resonances and the damage-induced resonances are excited more easily, a higher portion of the system energy is transformed on internal deformations and displacements. This means that the vibration behaviour changes can be interpreted as a reduction of the system performance and an increment in internal deformations. This is represented as:

$$
\begin{array}{r}
\text { Underperformance } \propto \text { Reliability for } T_{i}^{d} \\
f\left(\Delta F_{o p}\right) \propto f\left(\Delta H_{s t}, F_{d}, H_{d}\right)
\end{array}
$$

This means that the positive feedback loops responsible for failure divergence character (Sec. 3.2) correspond to the increased residual loads, as shown in Figure 4.3. 


\subsubsection{Failure behaviour}

An implication of equation 4.5 is that each failure state has a unique composition of $\Delta F_{o p}, \Delta H_{s t}, F_{d}$ and $H_{d}$, i.e. for each $T_{i}^{d}$ there is a corresponding $\Theta_{i}^{d}$. This implies that minor variations in $\Theta^{d}$ corresponding to the magnitude of the elements and their interactions are considered to be within state variations, whereas major variations are ascribed to transitions between states. A representation of failure progression through changes in the vibration is shown in Figure 4.10.

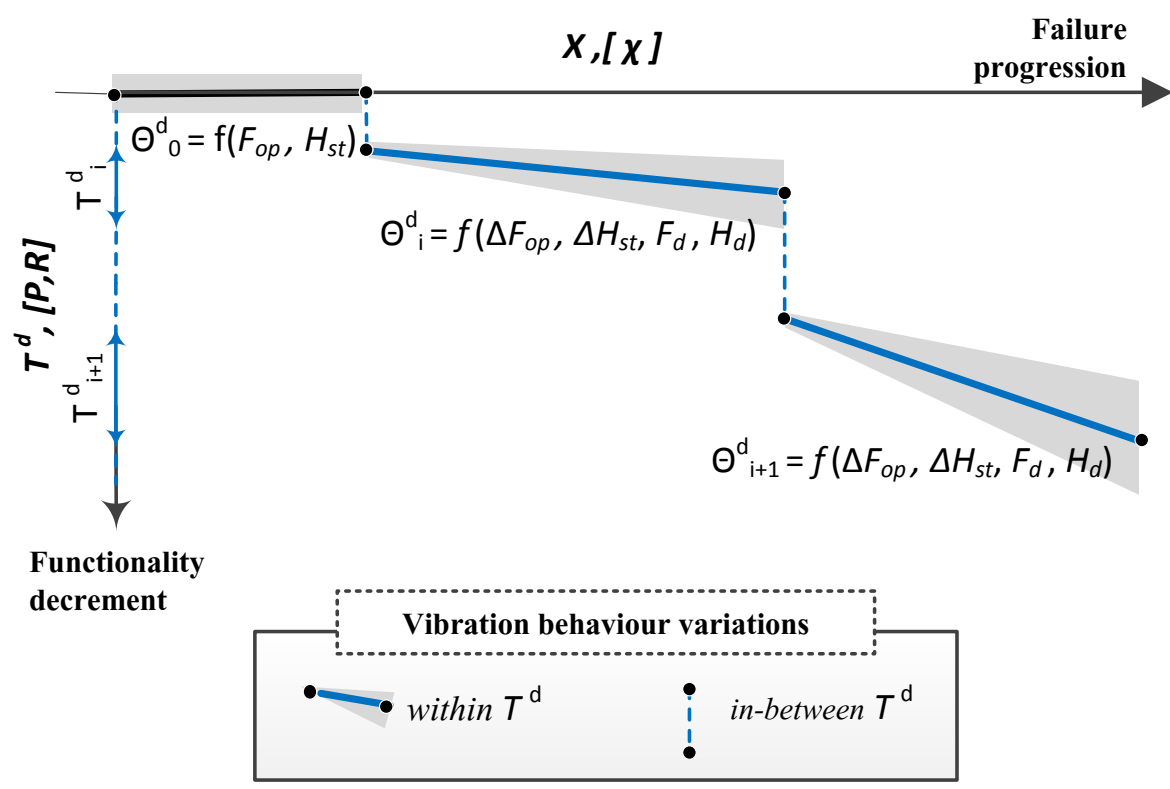

Figure 4.10: Failure progression characterisation by vibration analysis.

Figure 4.10 describes the evolution of failure as a function of the vibration response. The abscissa corresponds to the vibration response $X$, represented through the individual vibration modes $(\chi)$. The ordinate corresponds to the dynamics aspects of the system functionality $T^{d}$ evaluated through its performance and reliability. Three segments are identified. The first segment (black horizontal line) represents the designed state, whereas the two subsequent segments (blue inclined lines) represent two consecutive failure transformations $\Theta_{i}^{d}$ and $\Theta_{i+1}^{d}$. The segments are surrounded by a grey area that represents the variation of operational environment. 
Figure 4.10 also illustrates failure divergence and progressive character. Failure divergence is represented by the downwards trend for the failure states, both in relation to the slope of the failure states and the slump between the states. The progressive character is represented through the variations in the vibration. Variations within failure states are restricted to the functions $\Theta$ while variations between states are illustrated by the interruptions in the vibration (dashed lines). This implies that small transformations beyond the limits of the failure states have relatively large effects on the system functionality.

\subsection{Conclusion}

This chapter demonstrated that lateral vibrations are a direct indicator of the destabilisation forces responsible of the changes in the dynamic load distribution under complex failure. Yet, the interpretation of the vibration response requires of meaningful features that reflect the changes in the energy balance of the system according to the loss energy principle. A comprehensive, functional approach to the interpretation of the vibration response was proposed. This approach is based on the identification of the origins forces and resonances participating in the vibration response, the assessment of the nonlinear interactions reflected on the signals, and the evaluation of the system functionality. The functional approach will be demonstrated on next chapters for the cases of roller bearings and rotor blade systems. 


\section{Chapter 5}

\section{Uncertainty: Roller bearings}

\section{case}

\subsection{Introduction}

This chapter addresses two main objectives. Firstly, it discusses the uncertainty of the failure behaviour when the system is undergoing complex failure according to the kinetic energy principle. Secondly, it investigates the proliferation of a component failure mode to the system level. This is carried out for the case of roller bearing failure on different host systems. Both objectives are developed by the functional approach introduced in the previous chapter by identifying the elements and interactions responsible for the vibration response. Consequently, the load distribution is used to evaluate the effects of the component failure to the system level.

\subsubsection{Uncertainty principle and vibration behaviour}

As discussed in Section 1.2.1, rotating mechanical systems are designed following the assumption that the components' individual degradation paths are independent of each other. Thus, by defining the material load-carrying capacity, the system's expected use and the load distribution, every component's useful life could be predicted and hence engineered. In consequence, according to the independent failure assumption the overall system lifetime is defined by the lifetime of its main components. 
Nevertheless, the independent assumption does not consider the performance aspects of system degradation. Even in the absence of failure, the collaboration of individual component's degradation leads to lower performance at the system level, as seen in the hydraulic servo actuator in Example 4 (Section 2.1.2). Therefore, changes in load distribution increase the uncertainty about component's individual degradation path, and consequently the system failure behaviour as a whole.

The mechanism and dynamics of complex failure (Chapter 2 and Chapter 3) provide important conceptual elements to increase the understanding about the system degradation. Specifically, the loss energy principle points out the disruption of the kinetic energy transformation as the main driver of the failure process. Therefore, monitoring the factors associated with the kinetic energy is essential in order to characterise and predict failure development. This is stated in the kinetic energy principle as:

Kinetic Energy Principle Failure evolution depends on the destabilisation effect of kinetic energy with respect to the compromised functions, the physical damage characteristics and the external environment variability. Consequently, the likelihood of predicting failure evolution is conditioned to the uncertainties associated with each of these factors.

As discussed in Chapter 4, the lateral vibration response is a direct indicator of the destabilisation forces associated with failure. Consequently, the functional approach to vibration analysis enables the identification of the origins of the destabilisation forces (i.e. modified operation or damage-induced) and the characterisation of their variability with respect to the operational environment. This implies that, for a particular failure state, the treatment of the vibration behaviour must yield to a general description of the altered load distribution.

Based on Figure 4.10, the goals for failure behaviour characterisation through the vibration assessment are redefined for a specific failure state. The interpretation of the vibration signal problem, shown in Figure 5.1, is divided in two steps. Firstly, the characterisation of the altered load distribution $\Theta_{i}^{d}$ as a function of the changes in the operational forces $\Delta F_{o p}$, the changes in the structural resonances $\Delta H_{s t}$, the damage-induced forces $F_{d}$ and the damage-induced resonances $H_{d}$. Additionally, the assessment must indicate the variation of these elements with respect to the operational environment, as represented by the grey area.

Secondly, the identified load distribution must be interpreted with respect to effect of failure on the system functionality $T_{i}^{d}$. This could be used to predict the estimated useful life before reaching the maximum allowed functional degradation $T_{\max }^{d}$ in the absence of additional external disruptions. 


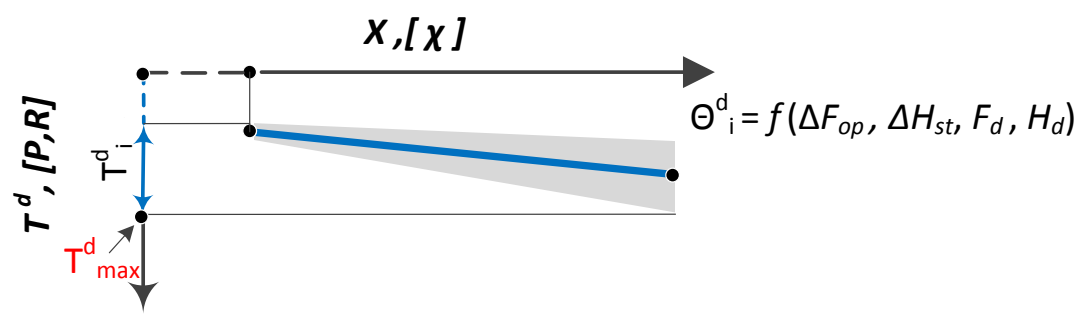

Figure 5.1: Characterisation of a failure state using vibration analysis.

\subsubsection{Roller bearing diagnostics}

Roller bearings are chosen to exemplify the variations of failure behaviour within a failure state. These components are critical for rotating mechanical systems as they provide support for the moving sub-assembly to the fixed frame. Most bearings are selected for an expected useful life equivalent to the main system and are thus not intended to be replaced during the service life. However, premature bearing failure is still one of the most common failure modes for rotating mechanical systems.

As discussed in Section 1.3, the problem of bearing damage assessment has been addressed from two different perspectives. The first approach corresponds to the early damage detection, which is supported by signal processing algorithms developed to isolate and extract the damage-related features against 'noisy' mechanical environments $[15,17,66,73-79]$. The second approach corresponds to the prognostics of remaining useful life based on machine learning and deep learning methods, which are supported mostly by generic features often without a predefined selection logic, also known as data-driven approaches [24,25,32,80]. As concluded before, the current approaches on bearing diagnostics lack physics insights about the proliferation from component to system level, thereby hindering the generalisability of the prognostics models. Furthermore, the current approach to bearing failure analysis considers surface damage as the starting point of failure. Yet, this focus offers limited information for assessing the conditions that lead to premature bearing failure.

This chapter demonstrates the functional approach to analysing the vibration response associated with bearing failure for different operating systems. This approach represents a starting point for the construction of predictive models for both component failure (prior surface damage) and system failure. 


\subsubsection{Chapter structure}

The analysis of bearing failure is developed through a comparative case study of the different kinetic energy factors that influence the bearing failure behaviour. The systems - lab set-up, wind turbine generator and train axle box - offer different functional contexts for evaluating the effect of bearing failure. Furthermore, the lab setup and the train case are used to investigate the different damage characteristics; and the wind turbine generator and the train axle box are used for the analysis of varying operating conditions. The cases are developed with the following objectives:

1. to evaluate the effect of damage on the bearing functionality with respect to context of the host system;

2. to evaluate the load distribution through the interactions between the driving forces and excited resonances, and

3. to characterise the variations of the vibration behaviour with respect to the factors that regulate the kinetic energy associated with the failure.

The chapter is organised as follows. Section 5.2 explains the complexity of roller bearings based on the interdependencies at component level and describes the vibration behaviour associated with bearing failure. The three cases are developed in the following sections. The lab set-up case presented in Section. 5.3 analyses the influence of the damage characteristics on the bearing functionality through the comparison of different artificial damages. The wind turbine generator case in Section 5.4 evaluates the vibration behaviour under highly variable operational loads. The train axle box case in Section 5.5 illustrates the effect of varying environmental loads. A summary of the findings for the different cases with respect to the proposed objectives is presented in Section 5.6. Finally, the conclusions about failure uncertainty are presented in Section 5.7. The details of the signal treatment used for each case are discussed in a separate Appendix A.

\subsection{Roller bearing failure behaviour}

\subsubsection{Functionality}

The main function of roller bearings is to provide support and separation to subassemblies of rotating mechanical systems with different rotational speeds. Roller bearing functionality can be described in line with the hierarchy proposed in Section 2.3.1: as a combination of the structural support function (Type II) for providing stable support to the rotating body, and the mitigation function (Type IV) for enabling 
free rotation. The two functions depend on different requirements, as illustrated in Figure 5.2.

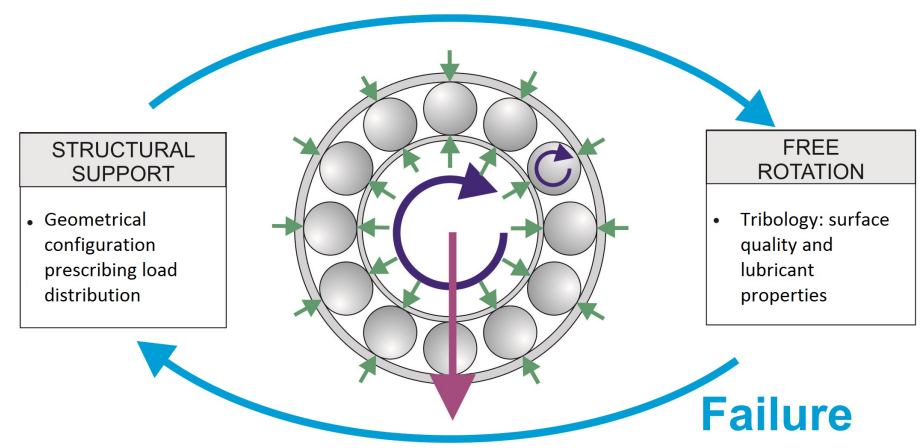

Figure 5.2: Bearing functions: structural support and free rotation.

The structural support function is defined by the geometrical configuration that ensures the load distribution, while the free rotation function is defined by the tribological conditions relating to the bearing surfaces and the lubricant $[81,82]$.

The stringent requirements for roller bearings lead to high design complexity. As an example consider the cage and seals that are optional elements of the bearing assembly. The cage ensures even separation of rolling elements for high stability, whilst the seals contain the lubricant inside the bearing and offer protection against external contamination. In this context the cage is related to the structural support function whereas the seals relate to free rotation. Thus a bearing configuration that enables high rotation speeds amidst a highly contaminated environment would require these two additional elements. Yet a higher number of elements at the bearing assembly carries a higher probability of simple component failure $[83,84]$.

\subsubsection{Bearing damage}

The normal degradation of bearings depends on the balance of the contact forces. Under normal operation, the lubrication conditions allow separation between the rolling elements and the bearing races while distributing the converging loads through the active rolling elements. Variations of the lubricant film, such as the thickness and viscosity, disrupt the equilibrium between normal and friction forces, affecting the structural support and free rotation functions simultaneously.

As mentioned before, surface damage starts the bearing failure as it affects the structural support function permanently. The causes of surface damage are attributed 
mainly to surface and sub-surface fatigue [81], but lubricant contamination is also not uncommon. In general it is possible to identify three degrees of surface damage:

Metal-to-metal contact: Increment in micro contacts on the surfaces for reasons such as deterioration of lubricant viscosity. The associated increment in friction forces affects the free rotation function. The resulting destabilisation of rotating shaft motion is minimal.

Surface deterioration: Definitive changes in the surface characteristics, which can be due to breakage of the micro-asperities associated with adhesive wear, or as built-in material due to particle contamination. Surface deterioration leads to the increment in the friction forces as well as lower stability support.

Superficial crack: Fracture of the running surfaces and subsequent removal of small discrete particles of material which can be due to abrasive wear, fatigue or thermal expansion. The generated cracks lead to sharp changes in the contact forces, disrupting the trajectory of the rolling elements and affecting the support function.

Each surface damage type leads to a unique dynamic and vibration behaviour. For instance, superficial cracks induce an impulsive disruption of the contact forces that excite the natural frequencies of the rolling elements. Worsening of the disruptions can lead to fatigue pits, hindering the restoring capacity of the contact forces and leading to a more random vibration behaviour [85].

The deterioration of each function affects the system differently throughout its operational life. Prior to surface damage, the free rotation function is important for the power transformation efficiency as bearings must comply with low energy consumption. After surface damage, the structural support function becomes more critical as its deterioration threatens the integrity of the rotating sub-assembly.

The disruption of the tribological interactions due to failure lead to a self-reinforcing process: small disruptions of the contact forces lead to more friction, which in time worsens the surface quality and triggers further surface deterioration. The increased friction contributes to a lower energy efficiency of the system, while the disruption of the contact forces changes the system configuration as local resonances emerge.

In sum, roller bearings are complex sub-systems within rotating mechanical systems, whose main roles correspond to the structural support and free rotation of the system. The normal degradation of bearings suppose an independence between these two functions as they rely to a large extent on different design requirements. Bearing failure due to surface damage triggers a complex failure behaviour within the component that proliferates to the system level in unanticipated ways. 


\subsubsection{Vibration behaviour}

Even though it is possible to explain the causes that lead to component failure (i.e. failure mechanism, failure modes and interdependencies), making a prediction of the effects at system level is more challenging. Vibration analysis provides an opportunity to assess the participation of the various forces and resonances on the failure behaviour.

Bearings are an intrinsic source of lateral vibration even in the absence of failure. This is because of the varying compliance due to the change in the active rolling elements at the loading zone [65]. These vibration levels are, however, limited so as not to compromise the stability of the bearing forces. Instead, surface damage causes severe distortion of the vibration responses. The irregularities cause abrupt changes in the contact loads, which generate short duration pulses affecting the elastohydrodynamic lubricated contacts and the dynamics of the structural components [82]. Figure 5.3 illustrates the impulse force and the corresponding response as the local bearing resonance is excited [86].

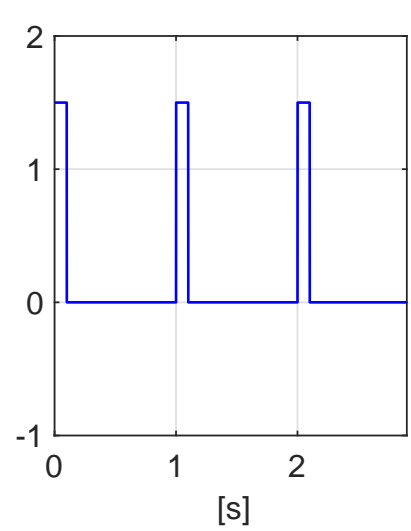

(a) Impulsive force

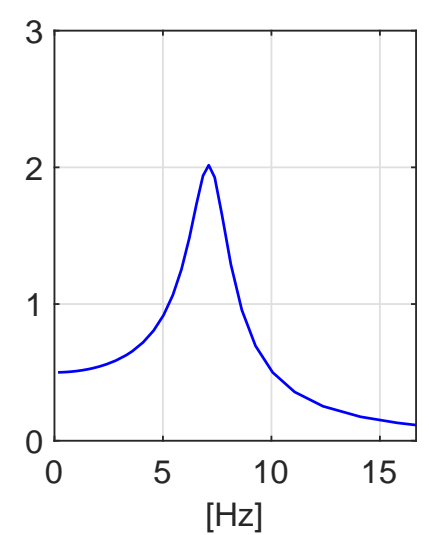

(b) Bearing local resonance

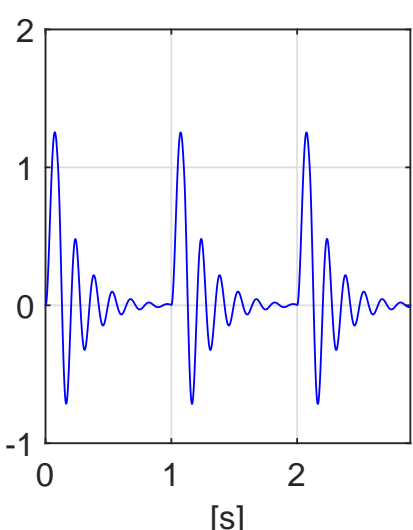

(c) vibration response

Figure 5.3: Example of impulse response.

As the size of the defect increases, the disruption of the contact forces is reflected in changes in the normal and tangential converging at the bearing $[66,87,88]$. From this moment onwards the system dynamic behaviour changes as a consequence of the altered load distribution. The following subsection present a model for bearing failure proliferation widely adopted in industrial practice. Subsequently, the considerations of the forces composition, resonances and operational environment 
variations on the signal processing are discussed.

\section{Generalised bearing damage model}

The first references on vibration failure behaviour identify four stages of bearing damage [85]. The spectral interpretation of each of these stages is presented in Figure 5.4.

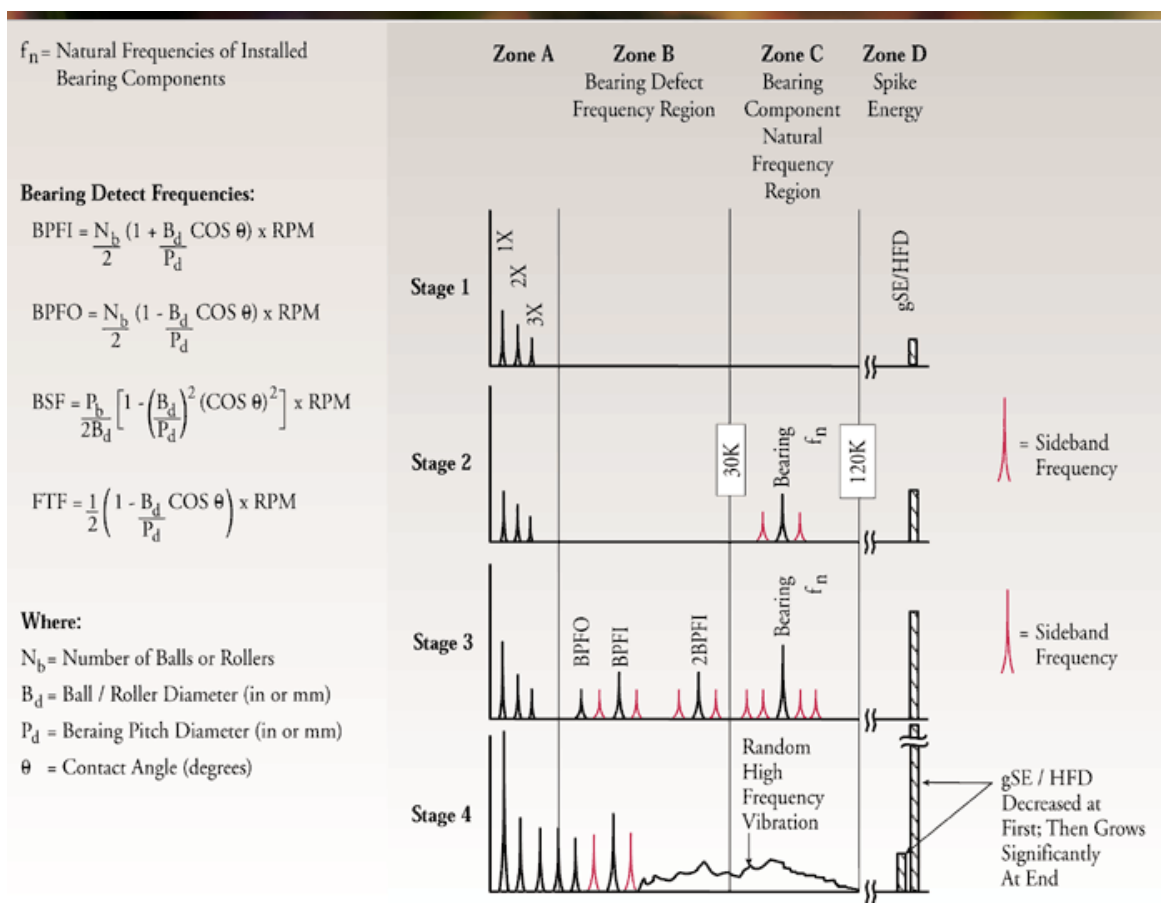

Figure 5.4: Stages of bearing damage ${ }^{1}$.

The model considers four main spectral areas of the system response.

Zone A corresponds to the lower (main) structural resonances $H_{s t}$ which are affected by the main operational forces $F_{o p}$ of the system.

\footnotetext{
${ }^{1}$ Taken from www.machinerylubrication.com
} 
Zone B corresponds to the higher natural resonances of the system $H_{s t}$ which are typically not excited at the normal operation. Additionally, it contains the recurrence frequency of the damage events $F_{d}$, also known as bearing failure frequencies $(\mathrm{BFF})$. Four BFF types are identified: bearing pass failure outer $(\mathrm{BPFO})$, bearing pass failure inner $(\mathrm{BPFI})$, rolling element deterioration frequency (BSF) and cage failing frequency (FTF).

Zone $\mathrm{C}$ contains the natural resonances of the bearing elements, or damage-induced resonances $H_{d}$ excited by the disruption of the contact forces $F_{d}$.

Zone $\mathbf{D}$ corresponds to the ultrasound range which is affected by sub-surface cracks and resonance of surface asperities.

Note that both Zone $B$ and Zone $C$ are related to the damage-induced forces. The main difference is that Zone $\mathrm{C}$ contains the actual impulse response while Zone $\mathrm{B}$ refers to the recurrence of the impact events. Furthermore, the model indicates four stages of damage.

Stage 1 shows that the abnormal vibration focuses at the Zone D while the normal residual vibrations reflect at zone $A$.

Stage 2 develops as result of superficial cracks exciting the natural frequencies of the bearing components $H_{d}$. Side bands corresponding to the damage recurrence $F_{d}$ appear in addition to to the local resonances.

Stage 3 corresponds to the increment in the magnitude of the impulsive forces $F_{d}$ affecting higher structural modes of the system $H_{s t}$. Meanwhile there is an increment in the operational forces $F_{o p}$ at the main lower operational resonances $H_{s t}$.

Stage 4 is marked by an increased activity in zones B and C, and significant disruption of the operational forces. When the magnitude of the impacts is low, the bearing restores its original state by means of the damping. However, if the bearing has not restored completely while another significant impact occurs, the leading edge of the new impact response is buried in the delay of the previous impact, leading to high stochastic behaviour. The behaviour of zone D is variable.

As described by the kinetic energy principle, failure evolution depends on the specific role of the affected components, the characteristics of damage and the variations in the operational environment. These influences can be traced back to the vibration response. The functional role is reflected in the characteristics of the operational forces $F_{o p}$. The damage characteristics concern the magnitude of the damageinduced forces $F_{d}$ and the extent to which they affect the different system resonances, $H_{s t}$ and $H_{d}$. Finally, the influence of the operational environment must be evaluated for all the individual elements of the vibration response. 


\section{Signal complexity}

The four-stages model offers a simple description of the proliferation of bearing damage. However, this model requires additional considerations of actual rotating mechanical systems such as the coexistence of multiple forces and resonances reflected in the vibration signal, and their variation due to the operating environment. As the cases presented in the following sections exhibit some of these characteristics, a preliminary indication of the signal processing approach is presented next.

Close resonance frequencies The identification of damage-induced modes $H_{d}$ becomes challenging when damage develops in a similar frequency range to $H_{s t}$. Damage can also change the mode shape and the eigenfrequency of the structural resonances $\Delta H_{s t}$, as will be seen for the railway environment shown in the train case. Adaptive filtering method such as wavelets and empirical mode decomposition (EMD) are recommended for the separation of signals with close frequency content.

Forces overlap Multiple forces can affect a single resonance simultaneously, as will be seen in the lab set-up case. Such overlap can be difficult to distinguish, specially if the magnitude of the forces is low. Demodulation methods such as envelope analysis are used to extract the most important forces for a defined frequency range. Additionally, the use of enhancing operators such as the signal power and the Teager-Kaiser Energy Operator (TKEO) emphasise the most representative sources of the vibration response. However, it should be noted that the use of enhancement operators disrupts the physical meaning of the signal, limiting its use for further quantification of the driving forces.

Non-stationary conditions Operation and environment are two different factors that influence the vibration behaviour of a system. In the wind turbine generator case, the main variation in the vibration response can be linked to the output current. For the train axle box case, the irregularities of the wheel and rail lead to a high variability on the vibration signal. In both cases, the variations affect the magnitude and frequency of the conveying forces and (main) resonances involved. Consequently, the vibration signals are analysed for relatively stable, yet short sample periods. The utilisation of Short-Time-Fourier-Transform (STFT) is recommended in these cases.

The three cases are developed in the following sections. Each case is analysed for the identification of the driving forces and excited modes participating in the vibration response. Next, the bearing functionality is evaluated with respect to the specific functional role of the component within the system. Finally, the load distribution changes are evaluated in respect of the kinetic energy factors. 


\subsection{Case I: Lab set-up}

The first case corresponds to a lab set-up developed by the Fiat Research Centre [89]. This set-up enables the characterisation of damage at different elements of the bearing assembly. The set-up consists of a low power electric motor driving a pulley connected by a belt transmission at constant speed and load, as seen in Figure 5.5. The sensing accelerometer was located at the pulley at the vertical direction.

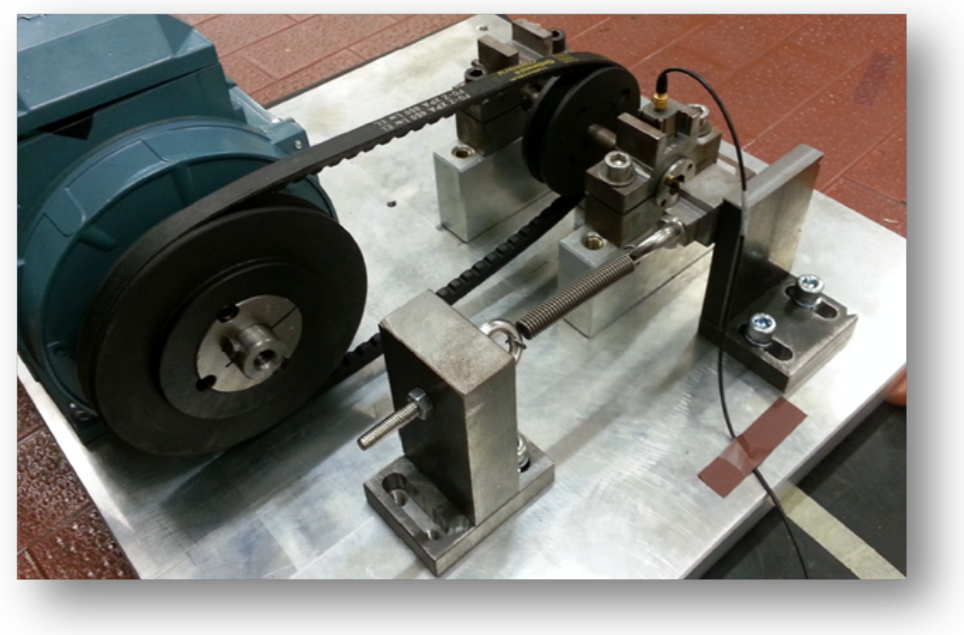

Figure 5.5: Lab set-up for artificial damage evaluation. ${ }^{2}$

The driven pulley is supported by the test bearing, a 6205 deep groove ball bearing, modified with single point faults (electro-discharge machined $0.18 \mathrm{~mm}$ ) to simulate surface crack damage. No other changes in the loading conditions that affected the operational forces and rotational speed were reported. The defects were introduced at the outer race, the inner race and the rolling elements. The test conditions and the bearing failure frequencies ${ }^{3}$ are presented in Table 5.1.

Each damage condition was recorded for up to 10 seconds at a sampling frequency of $12 \mathrm{kHz}$. During the test duration the signals display no variations on the rotational frequency. Differing rotating speeds were, however, observed for the different test conditions, for an overall range between 1700 to $1800 \mathrm{rpm}$, which was confirmed by the spectral analysis as reported later in the analysis.

\footnotetext{
${ }^{2}$ Taken from Cisi et al [89]

${ }^{3}$ Source: SKF online calculator www.skf.com
} 
Table 5.1: Test information for lab set-up case

\begin{tabular}{|l|l|l|l|}
\hline \multicolumn{2}{|c|}{ Test Information } & \multicolumn{2}{l|}{ Failure frequencies [orders] } \\
\hline Bearing type & $6205-2 \mathrm{RS} \mathrm{JEM}$ & Outer race & 3.585 \\
\hline Damage size & $0.18 \mathrm{~mm}$ & Inner race & 5.415 \\
\hline Sampling speed & $12 \mathrm{kHz}$ & Roller element & 4.715 \\
\hline Rotational speed & $1700-1800 \mathrm{rpm}$ & & \\
\hline
\end{tabular}

The type of lab set-up and procedure presented in this section is common to several studies on validating signal processing methods of early damage detection [17, 90]. Nevertheless, for the scope of this paper, the emphasis is on estimating the different damage locations for the component functionality as evaluated according to the structural support and free rotating criteria.

\subsubsection{Signal characterisation}

Table 5.2 presents a comparison between the reference and the various damage cases. The time signal from the reference case shows seemingly-random behaviour since no strong periodicities are visible. Instead the outer race damage signal shows welldefined impulses as expected from surface-crack-induced impulses. Similarly the inner race damage signal reveals impulsive response yet is less defined than the outer race case. The rolling element damage shows a greater amplitude level and more spacing between impacts than in the other cases involving damage.

\section{Modes identification}

The distribution of the energy content is shown in the spectrograms of Table 5.2. These show that for the undamaged case, the normal vibration activity focuses below $2 \mathrm{kHz}$. All the cases involving damage reveal a higher concentration of vibration activity between $2 \mathrm{kHz}$ and $4 \mathrm{kHz}$, corresponding to the damage-induced resonances.

The composition of the damage-induced resonances provides additional information about the characteristics of the surface damage. Figure 5.6 shows a detail of the entry and exit of the passing of the ball over the outer race. The separation between these events can be used to estimate the crack size [78]. 
Table 5.2: Input signals and spectrograms for lab set-up case.

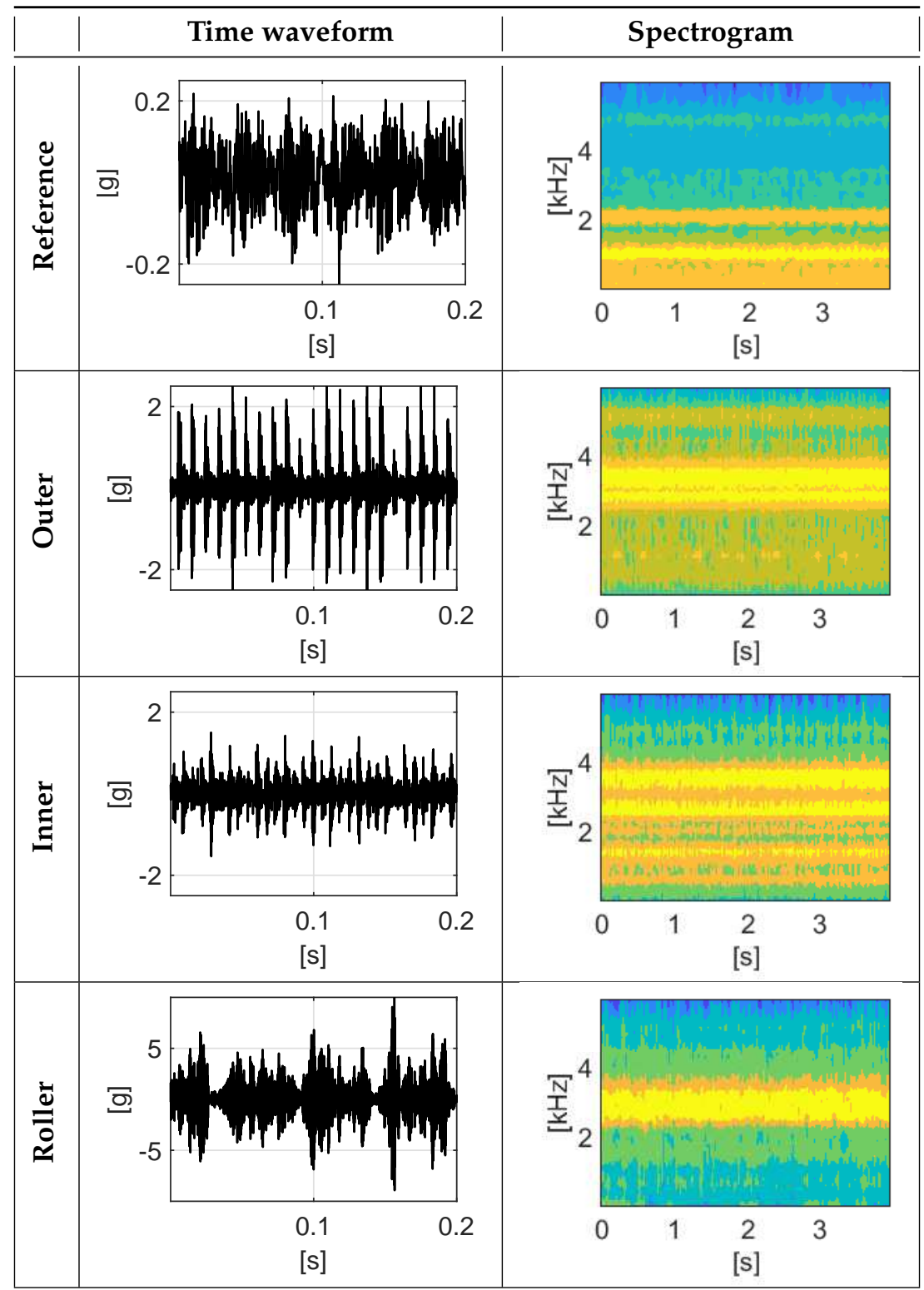




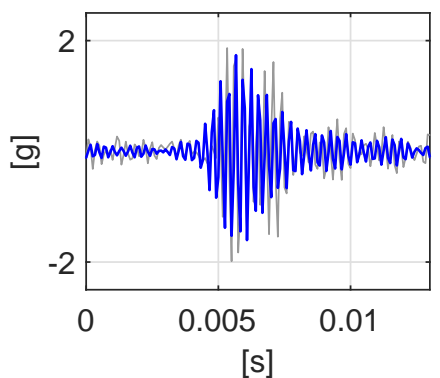

(a) entry

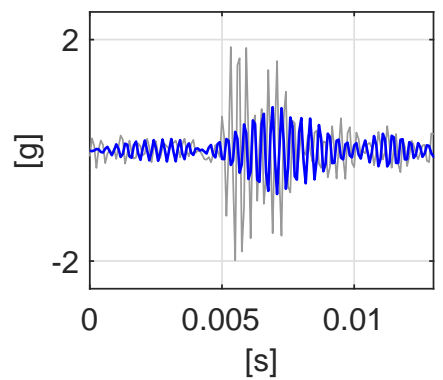

(b) exit

Figure 5.6: Crossing of the ball element passing over the outer race crack. The grey signal corresponds to the original signal. The blue signal corresponds to the filtered signal between 2.5 to $3.2 \mathrm{kHz}$ for the entry and between 3.2 to $4 \mathrm{kHz}$ for the exit.

\section{Force identification}

The nature of the operational forces $F_{o p}$ and damage-induced forces $F_{d}$ is discussed next. For the lab set-up case, the main source for $F_{o p}$ is likely to be the imbalance forces at the pulley, although other forces associated with the rotational speed of the belt can be expected. The recurrence of the impulsive forces $F_{d}$ is estimated by the BFF multipliers presented in Table 5.1 times the rotational frequency.

Despite already knowing the expected force frequencies, identifying them by means of spectral analysis is not always straightforward. A comparison between three methods of identifying driving forces is presented in the following paragraphs based on the Fast Fourier Transformation (FFT), the envelope analysis and the TeagerKaiser Energy Operator (TKEO). These methods are illustrated through the case of inner race damage, with the rotating frequency (ROT) estimated at $28 \mathrm{~Hz}$ and the bearing failure frequency (BFF) at $155 \mathrm{~Hz}$.

The first method corresponds to the direct identification at the frequency spectrum. The time waveform and the spectrum are shown in the Figure 5.7. The time signal reveals a periodicity on the impulsive events whereas the spectrum shows a high peak around $590 \mathrm{~Hz}$ (green marker) related to a high structural frequency. Two groups of side bands are identified. The first group (blue marker) corresponds to $F_{o p}$. The second group (red marker) corresponds to the BFF frequency attributed to $F_{d}$.

The second method of extracting the modulating frequencies is the demodulation envelope shown in the lower frames of Figure 5.8. This method is based on a constructed envelop signal over the rectified time signal, which serves as a band pass 


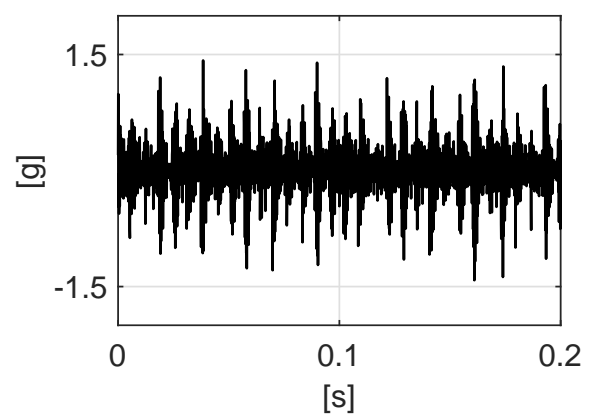

(a) Time waveform

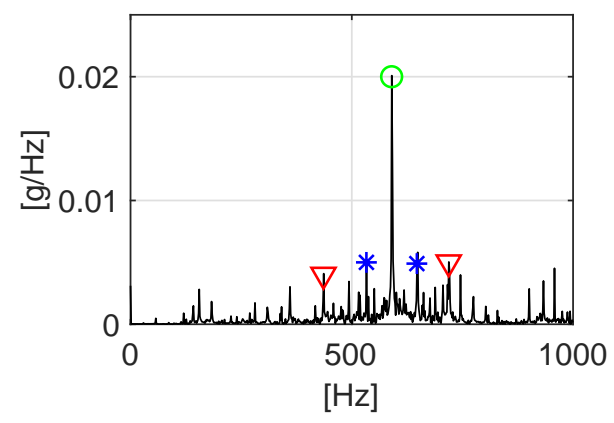

(b) Spectrum

Figure 5.7: Identification of driving forces at the spectrum.

filter around the local resonance frequency [91]. The envelope spectrum shows the two frequencies corresponding to $F_{o p}$ and $F_{d}$. This means that $F_{o p}$ and $F_{d}$ exert a combined influence on $H_{d}$.

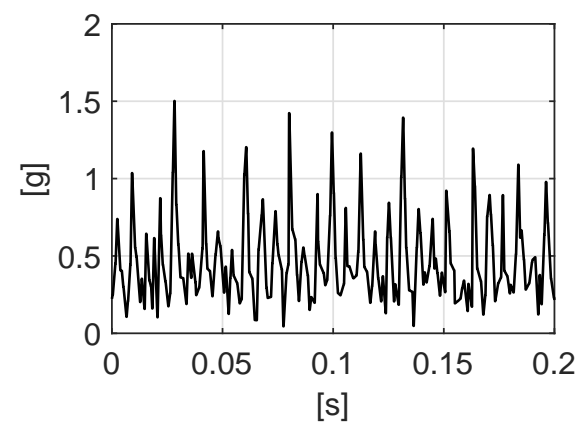

(a) envelope waveform

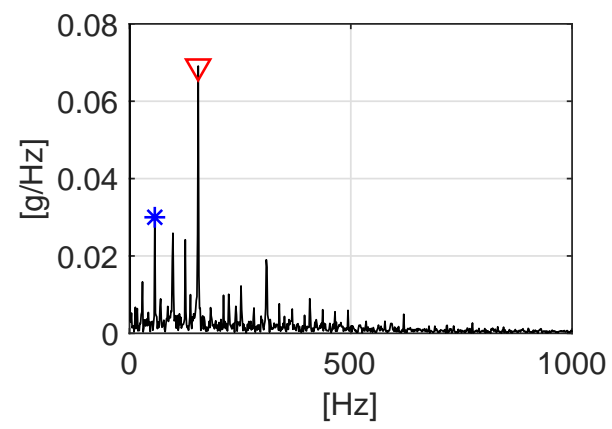

(b) Spectrum

Figure 5.8: Envelope analysis for identification of driving forces.

The last method reviewed is based on the Teager-Kaiser Energy Operator (TKEO). This operator enhances the signal to reveal the most relevant modulating phenomena. The TKEO enhancer is based on the concept of total energy of a simple oscillator, and follows a formulation similar to the Lagrange operator [92,93]. However, the physical meaning of the vibration acceleration signal is less evident, hence it will be used only for comparison purposes. Nevertheless, the TKEO operation is used as an indicator of amplitude and frequency modulation of mechanical systems [94]. 
The TKEO operator is applied to two filtered signals corresponding to the structural resonances $H_{s t}(0 \mathrm{~Hz}$ to $1000 \mathrm{~Hz})$ and the resonances $H_{d}(2300 \mathrm{~Hz}$ to $3800 \mathrm{~Hz})$ as shown in Figures 5.9 and 5.10. Note that similarly to the envelope waveform (Figure 5.8a) the TKEO waveform also leads to a positive signal. By applying TKEO over the filtered signals it is possible to identify the influence of the different forces over the selected resonances more clearly. The peak values for the operation and forces are highlighted respectively by blue and red markers.

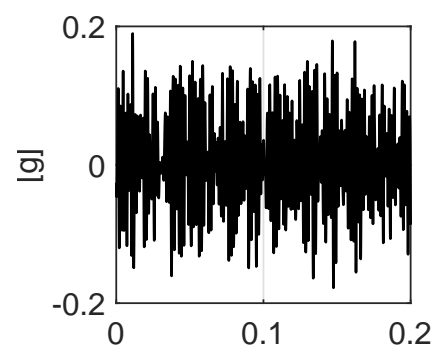

[s]

(a) Time waveform

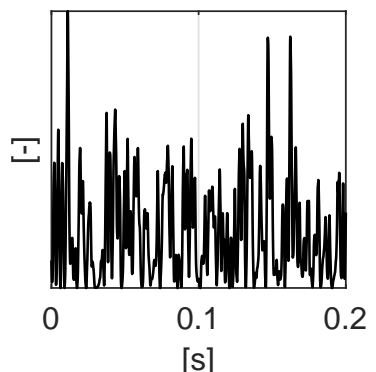

(b) TKEO waveform

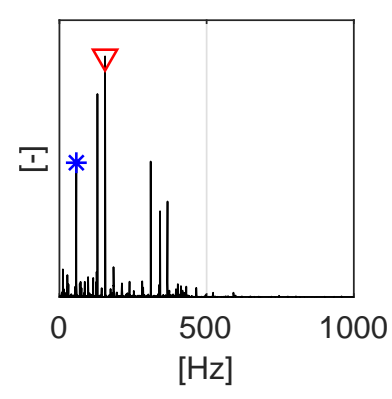

(c) TKEO spectrum

Figure 5.9: TKEO analysis for signal filtered at $H_{s t}$.

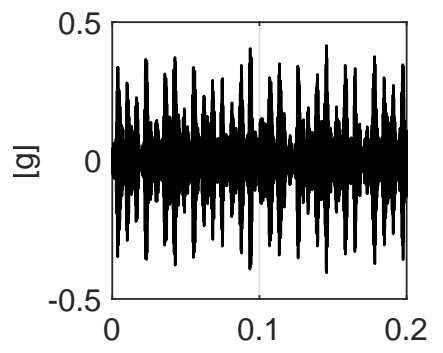

[s]

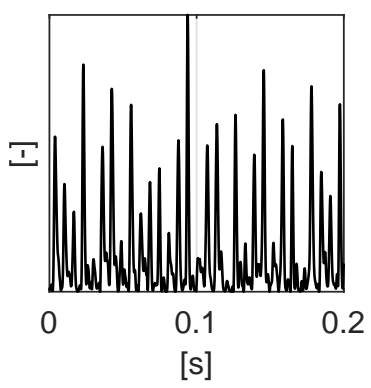

(b) TKEO waveform

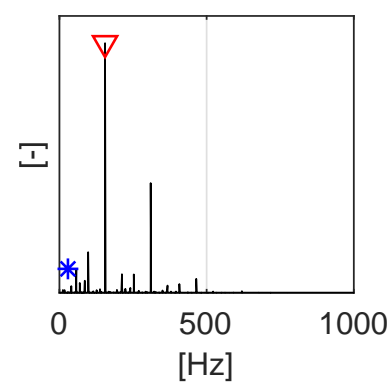

(c) TKEO spectrum

(a) Time waveform

Figure 5.10: TKEO analysis for signal filtered at $H_{d}$.

An overview of the filtered signals and the TKEO spectra for the reference and the damage cases is shown in Table 5.3. 

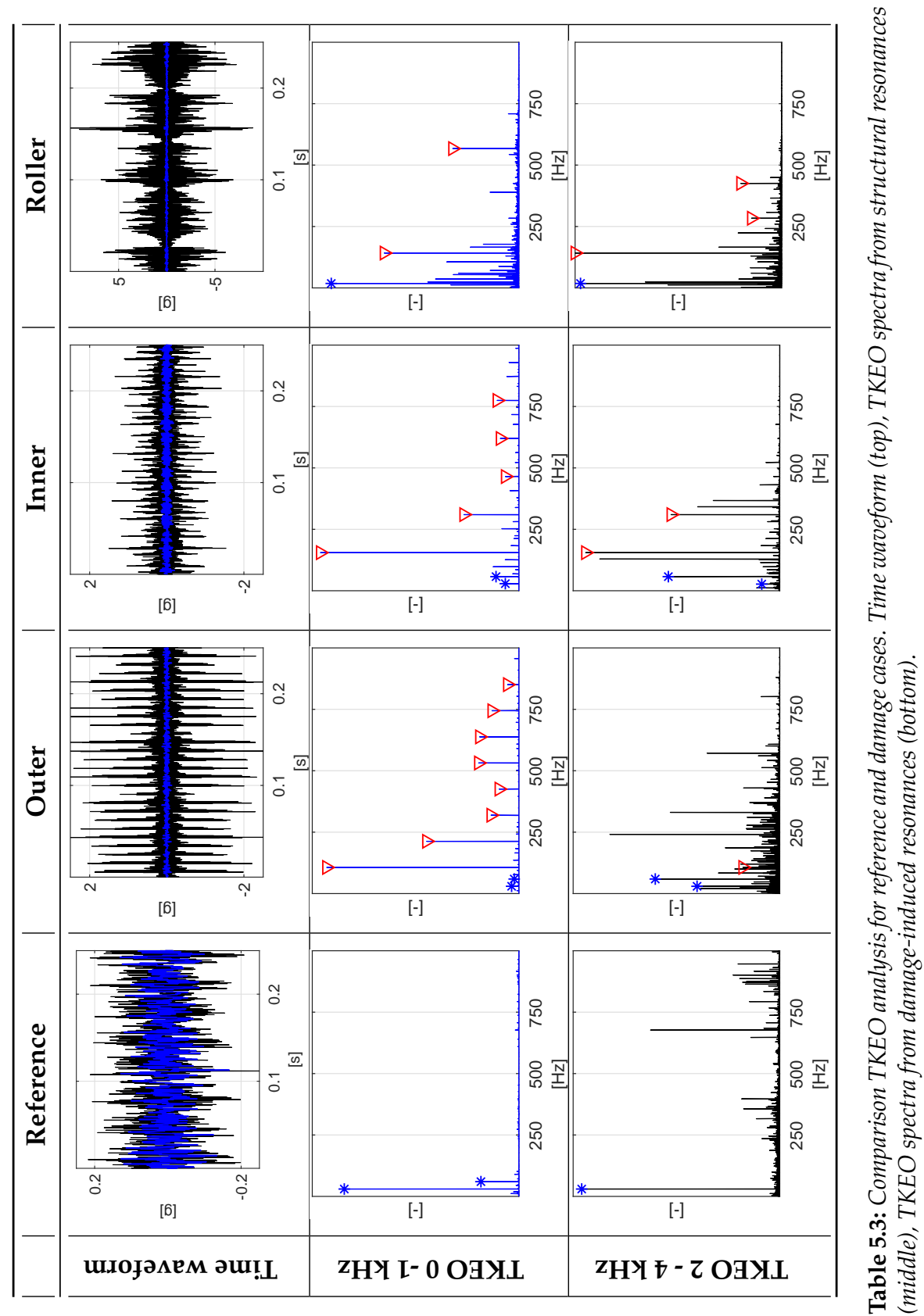
Furthermore, the identified frequencies are used to verify the rotational speed and bearing failure frequencies. Table 5.4 presents the modulation frequencies derived from the TKEO. The frequency of the most significant operational forces and damage-induced forces were extracted from the TKEO spectra directly. The rotational speed is derived from the $\mathrm{BBF}$ and the corresponding multiplier shown in Table 5.1.

Table 5.4: Derived frequencies for operational and damage-induced forces.

\begin{tabular}{lccc}
\hline & $\begin{array}{c}\mathbf{F}_{\text {op }} \\
{[\mathbf{H z}]}\end{array}$ & $\begin{array}{c}\text { Rotation } \\
{[\mathbf{H z}]}\end{array}$ & $\begin{array}{c}\mathbf{F}_{\mathbf{d}} \\
{[\mathbf{H z}]}\end{array}$ \\
\hline No damage & 30 & 30 & - \\
Outer & 29.7 & 29.67 & 106.4 \\
Inner & 28.8 & 28.62 & 155 \\
Roller & 18 & 30.1 & 141.9 \\
\hline
\end{tabular}

Table 5.4 shows that in the case of inner race and outer race damage the operational and the rotational frequencies coincide. Conversely, the case of roller element damage shows a difference between the operational frequencies and the estimated rotational frequencies. This indicates that the compromised operational forces are related to other components of the test set-up, e.g. the belt transmission rather than the bearing's own imbalance.

In summary, the different conditions analysed in this case follow a general pattern in the vibration response, described as:

$H_{s t}$ : The main structural modes are identified below $1 \mathrm{kHz}$.

$F_{o p}$ : The operational forces correspond to the inertial forces of the pulley. Yet for the roller element damage, the system displays a different frequency not related to the bearing rotation.

$H_{d}$ : The damage-induced resonances were identified between $2 \mathrm{kHz}$ and $4 \mathrm{kHz}$. The composition of these modes reflects the specifics of the passing over the race surface crack.

$F_{d}$ : The damage-induced forces were found consistent with the expected bearing failure frequencies.

The next two sections discuss the interpretation of the modes and forces previously identified on the system functionality. 


\subsubsection{Functionality evaluation}

The evaluation of the bearing damage is carried out through a set of generic vibration features representing the damage characteristics and functional effects, as presented in Figure 5.11. The damage features correspond to the overall vibration level and the peak amplitude, the latter as an indicator of the impulsive forces as consequence of the disruption of the contact forces (Figure 5.11a). The functional features relate to the evaluation of the structural support and free rotation bearing functions (Figure 5.11b). The structural support function is evaluated according to the overall vibration for the operational frequency range. The free rotation function is evaluated according to the instantaneous rotational speed, as extracted from the TKEO spectra.

The two damage features display similar behaviour as they are, as expected, minimal in the reference case, have a moderate level in the outer and inner case, and are maximal in the case of roller element damage. Furthermore, the outer race damage displays higher overall values than the inner race damage.

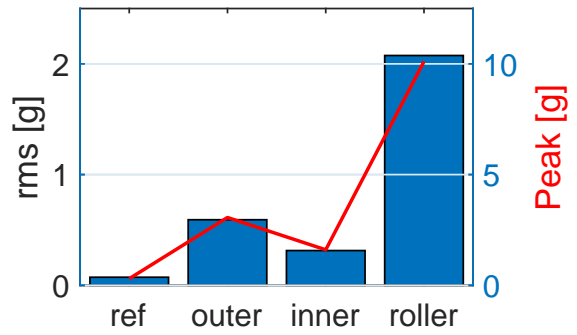

(a) Damage

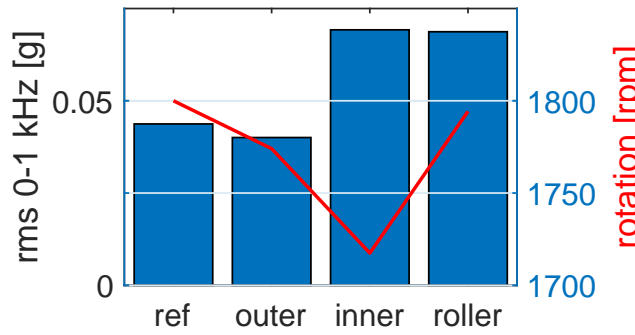

(b) Functional

Figure 5.11: Feature comparison for the reference and damage cases.

The functional features present a different trend from that of the damage features. The overall vibration shows a similar value for the reference case and for the outer race case, implying that this damage time does not have a strong effect on the support function. Conversely, the inner and roller element cases show higher rms values in comparison. Furthermore, the variation of the rotational speed shows stronger effects in the case involving inner race damage.

The comparison between the outer and inner race damage stresses the additional information provided by the functional features. The higher vibration values for the operational range relating to the inner damage suggest that the system is structurally more affected when damage occurs closer to the rotating elements. Similarly the decrease of rotational speed shows that the inner damage leads to higher losses in the rotational speed than for the outer case. This can be explained as the impacts at the 
inner race occur more often than at the outer race (see Table 5.1). However, the damage features point to a different trend as the outer race displays higher overall and peak values than the inner race. This could be interpreted that the outer case damage is more severe that the inner case; however, this occurs because of the shorter transmission path between the damage location and the sensor.

The damage at the rolling element also displays some apparent contradictions when analysed following the two approaches. Despite displaying considerably higher values for the overall and peak values, as shown in Figure 5.11a, the vibration in the structural range is comparable to the inner race case. Furthermore the rotational speed displays little variation with respect to the pristine case. However, the fact that the operational frequency identified for this case (see Table 5.4) does not correspond to the bearing rotation suggests that the failure has proliferated to beyond the bearing. Given the lower reported frequency $(18 \mathrm{~Hz})$, this frequency is likely to be related to the belt rotation.

\subsubsection{Load distribution evaluation}

The change in the load distribution is assessed through the peak values of $F_{o p}$ and $F_{d}$ from the TKEO spectra from Table5.3. The logarithm of the TKEO values for the operational forces $F_{o p}$ are shown in Figure 5.12a, for the structural and the damageinduced resonances $H_{s t}$ and $H_{d}$. Note that for the reference condition the operational forces are also shown at the higher frequency range, but in a lower magnitude than the damage cases. The roller element damage shows the highest effect at the damage-induced resonances. The sharp increase in the overall vibration values and the different operational force indicate that the roller element damage may correspond to a different failure state than the inner and outer race damage cases.

Similarly, the TKEO exponents for the damage forces $F_{d}$ are shown in Figure 5.11b. It is seen that for the outer damage the forces are lowest at the structural range. This is explained by the greater distance to the rotating assembly.

In sum, the load distribution as revealed through the vibration response can be described as:

$$
X_{l a b}=\left(H_{s t}+H_{d}\right) \cdot \Delta F_{o p}+\left(H_{s t}+H_{d}\right) \cdot F_{d}
$$

where the symbol $\Delta F_{o p}$ emphasises the change in the magnitude of the imbalance forces in the inner and outer cases, and in a different operating force in the case of roller element damage. Also the frequency range for the structural resonances ( $<$ $1 \mathrm{kHz})$ is much lower than for the damage-induced resonances $(2-4 \mathrm{kHz})$. 


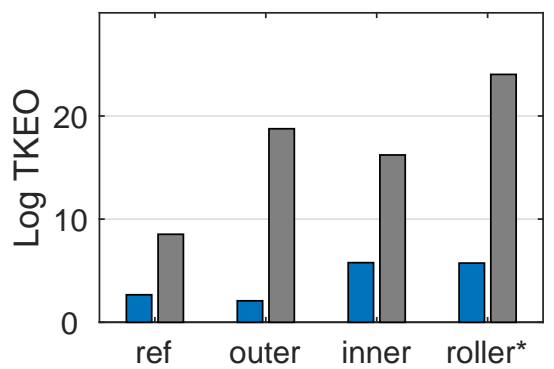

(a) Operational forces

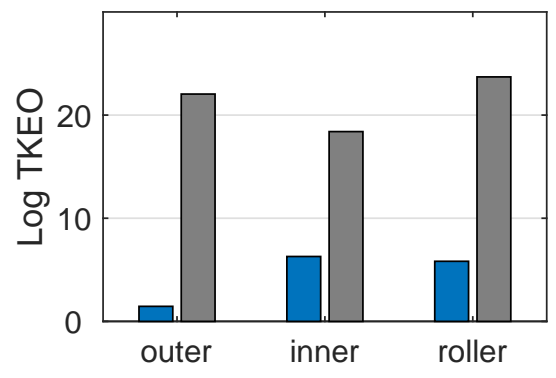

(b) Damage induced forces

Figure 5.12: Load distribution assessment by TKEO exponents over $H_{s t}$ (blue) and $H_{d}$ (grey).

This case showed that even though the magnitude of the damage was similar, the location differences lead to very distinct effects on the system functionality and the associated load distribution changes. The differences between the outer and inner case turn out to be quantitative only when they excited the equivalent operational and damage-induced forces. The inner case appeared to be the most significant for the disruption of the operational forces, while in the outer case the vibration activity remained comparatively more focused at the damage-induced resonances. Conversely, the damage at the roller element shows a qualitatively distinct case from the others.

\subsection{Case II: Wind turbine generator}

The second case analyses a wind turbine generator with bearing damage. This case was developed to characterise the influence of the operational load variations on the failure behaviour. Wind turbine generators (Figure 5.13) are rotating mechanical systems required to transform the kinetic energy extracted from the wind resource into electrical power to be delivered to the grid. In general, generator bearings must provide high stiffness so as to ensure the stability of the electromagnetic field between the static part and the rotor. This requirement is even more stringent for wind turbines, as they are exposed to transient loads due to the nacelle motion, the variations in the wind resource and the effect of grid synchronisation [95].

Generator bearings are designed for a lifetime similar to the entire turbine assembly, yet their premature failure is not uncommon. Component replacement is often delayed due to the restrictions for accessing wind turbines, as in the offshore environment. Consequently, in many cases bearing failures are left to progress until 


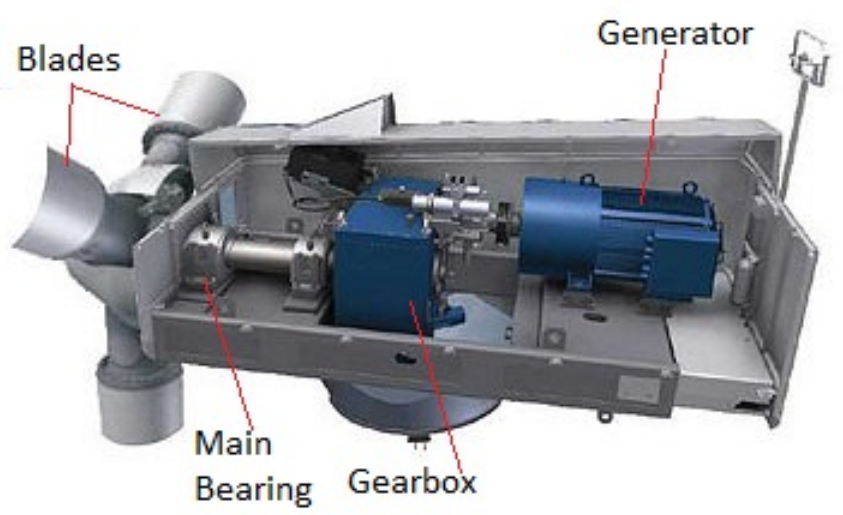

Figure 5.13: Wind turbine drive train.

adequate conditions are in place. Under these circumstances, vibration monitoring is essential in order to prevent damage increasing to dangerous levels that cause catastrophic failures in the entire wind turbine [96,97].

The specific case used in the following analysis corresponds to a doubly fed induction generator for which damage at the drive end was reported. Two vibration signals are used for the analysis. These correspond to the sixth month (M6) and tenth month (M10) after the damage was initially detected. Additionally the output current at the generator terminals was recorded, and it is used as an indicator of the instantaneous generated power. Further bearing information is presented in Table 5.5.

Table 5.5: Bearing information for wind turbine generator case.

\begin{tabular}{|l|l|l|l|}
\hline \multicolumn{2}{|c|}{ Test Information } & \multicolumn{2}{l|}{ Failure frequencies [orders] } \\
\hline Bearing type & 6320 & Outer race & 3.073 \\
\hline Sampling speed & $20 \mathrm{kHz}$ & Inner race & 4.927 \\
\hline Max. rotating speed & $1947 \mathrm{rpm}$ & Roller element & 4.927 \\
\hline
\end{tabular}




\subsubsection{Signal characterisation}

The current and vibration signals are presented in Table 5.6. The signals were recorded with a sampling frequency of $20 \mathrm{kHz}$ and for a duration of 20 seconds for M6 and 40 seconds for M10.

Table 5.6: Input signals and corresponding spectrograms for wind turbine generator case.

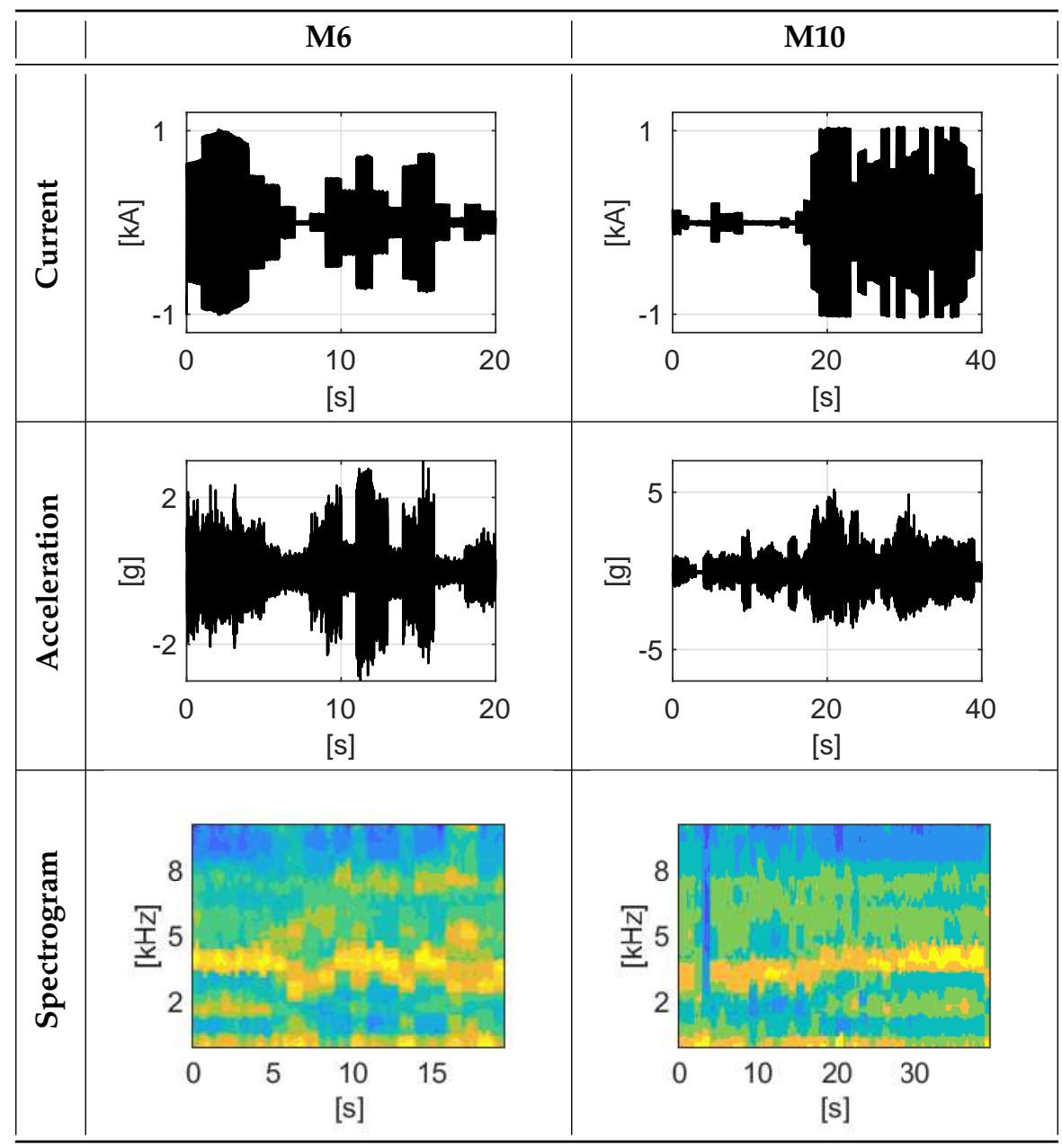


The vibration signal shows strong non-stationarity which can be traced back to the variations in the current. Wind turbines regulate the output power from the continuously changing wind resource through current (up to 1000 amperes) while the voltage level is kept constant. The specific case analysed shows an abnormal variation in the current signal, which indicates probable network instability or regulation problems.

The comparison between the vibration and current signals shows that at month six the vibration behaviour follows the current changes more closely than at month ten. In addition, the vibration at month ten displays increasingly higher values and more diffuse behaviour.

\section{Modes identification}

The frequency distribution for the two vibration signals is presented in the lower frames of Table 5.6. Similarly to the lab case, there are two marked regions of vibration activity. The highest concentration appears between $2 \mathrm{kHz}$ and $5 \mathrm{kHz}$, corresponding to the damage-induced resonances. The structural vibration is concentrated below $500 \mathrm{~Hz}$, which is significantly lower than in the lab set-up. This is because the inertia of the generator rotor is greater than in the lab set-up case.

\section{Force identification}

The TKEO modulation was performed to identify the driving forces over the two frequency ranges found. Given the high variability in the signal, short segments of 0.4 seconds were used for the analysis. Figure 5.14 shows the main peak frequencies found at the TKEO spectra for the structural and damage-induced resonances at M10.

Figure 5.14a shows a prevailing peak presence at $100 \mathrm{~Hz}$ at the structural resonance. This frequency corresponds to twice the electrical frequency (i.e. $2 \times 50$ $\mathrm{Hz}$ ), indicating that the most relevant operational forces reflected in the vibration behaviour are of an electrical nature. No imbalance forces were identified at the rotation speed in the expected frequency range (below $31 \mathrm{~Hz}$ ).

Figure 5.14b shows a more consistent occurrence at $127 \mathrm{~Hz}$, attributable to the damage-induced forces. Note that only half of the total sample was used, as for the first part the low current values did not lead to a converging pattern. Furthermore, the type of bearing damage associated with the identified frequency could not be matched, as it was not possible to detect the rotational frequency of the generator. 


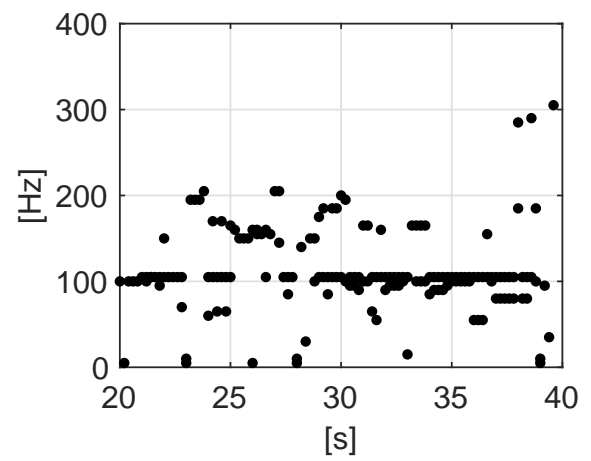

(a) structural resonances

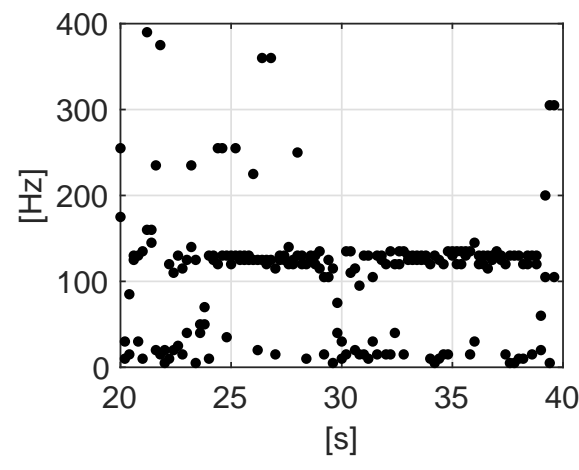

(b) damage-induced resonances

Figure 5.14: Peak frequencies of TKEO spectra at M10.

In summary, the changes in the operational loads restricted the identification of the driving forces through direct observation at the FFT spectrum. Instead, the TKEO analysis confirmed the presence of two distinct driving forces. Yet the analysis of the vibration response shows that:

$H_{s t}$ : Although the vibration activity associated with the main structural resonances remains below $500 \mathrm{~Hz}$, the transient vibrations indicate variations in the participation of these modes.

$F_{o p}$ : The most relevant forces exiting the main structural resonances are of an electrical nature. Mechanical imbalance forces were not revealed as discrete peaks in the TKEO spectra.

$H_{d}$ : The damage-induced resonances occur between $2 \mathrm{kHz}$ and $5 \mathrm{kHz}$.

$F_{d}$ : The damage-induced forces reveal at around $127 \mathrm{~Hz}$ but mainly at the periods where the current output is sufficiently high.

\subsubsection{Functionality evaluation}

The structural support function for the wind turbine generator bearing is evaluated in respect of the current value. Figure 5.15 presents a scatter plot of the rms vibration for the structural range with respect to the current for months six and ten. The signal was filtered for frequencies below $10 \mathrm{~Hz}$, as recommended for wind turbine components [98].

The vibration distributions show a linear correlation with respect to the current. However, month ten shows an outlier at the highest end of current value. This singu- 


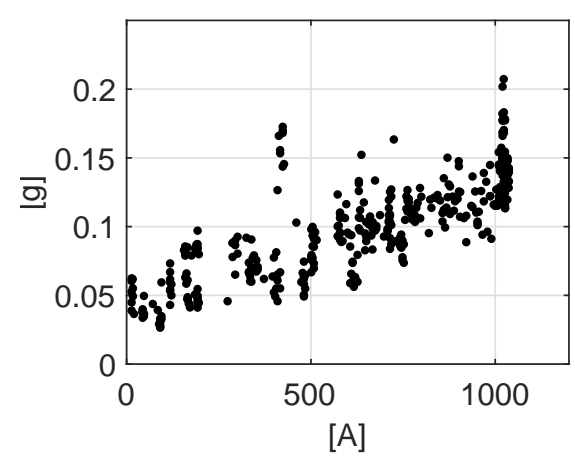

(a) $M 6$

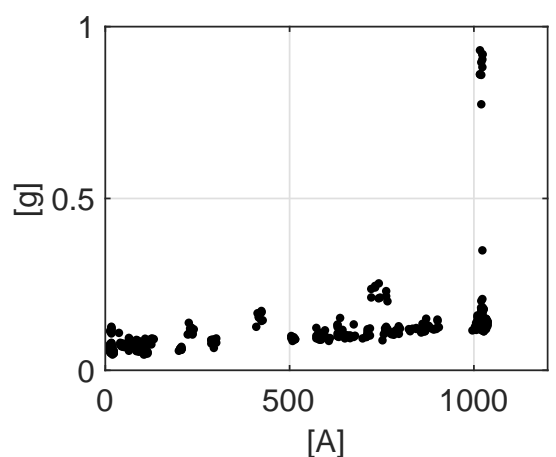

(b) $M 10$

Figure 5.15: Evaluation of the structural support function with respect to the output current.

larity is in accordance with a high mean value taking place for a few seconds during a period of high current. Prolonged exposure to high amplitude and asymmetric vibrations can lead to severe disruptions in the electromagnetic field and accelerated thermal degradation of the coils [99].

A linear regression for the relation between output current and vibration response is carried out excluding the outliers shown in M10. The coefficients and offset of the linear regression, and the dispersion $R^{2}$, are presented in the 5.7. It can be seen that the major variation occurs at the offset of the line while the slope of the regression remains stable.

Table 5.7: Linear regression parameters

\begin{tabular}{lccc}
\hline & Coefficient & Offset & $\mathbf{R}^{\mathbf{2}}$ \\
\hline M6 & 7 E-5 & 4.4 E-2 & 6.2 E-1 \\
M10 & 7 E-5 & 6.3 E-2 & 8 E-1 \\
\hline
\end{tabular}

\subsubsection{Load distribution evaluation}

As seen from the uneven damage-induced forces participation and the outliers at the functionality evaluation, the changes in the power output lead to instabilities in the vibration response. These instabilities demonstrate the need for a classification of the signal that enables a fair comparison of the load distribution changes between M6 
and M10. Four clusters are defined based on the current amplitude (low, medium and high) and an additional category for the outlier events with the highest mean values (non-linear). The details of the classification are presented in the Appendix A.

Figures 5.16 and 5.17 show the resulting clusters for the two acceleration signals. The left frames show the different categories at the time waveform. The right frames present the relation of the total acceleration with respect to the feature $f_{50}$. The feature $f_{50}$ refers to the frequency at which the spectral distribution reaches fifty per cent $(50 \%)$ of the overall rms value, hence it helps as an indicator of the energy distribution across the frequency spectrum.

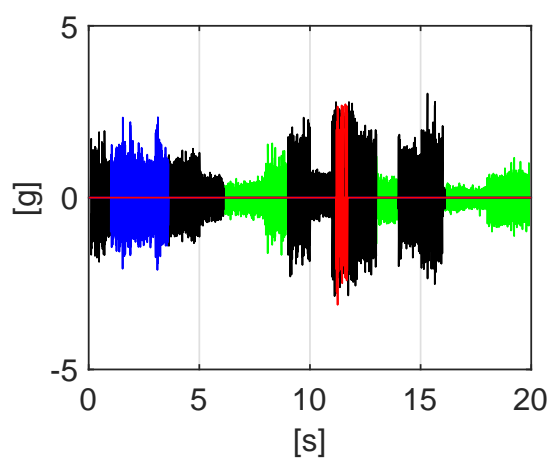

(a) Time waveform

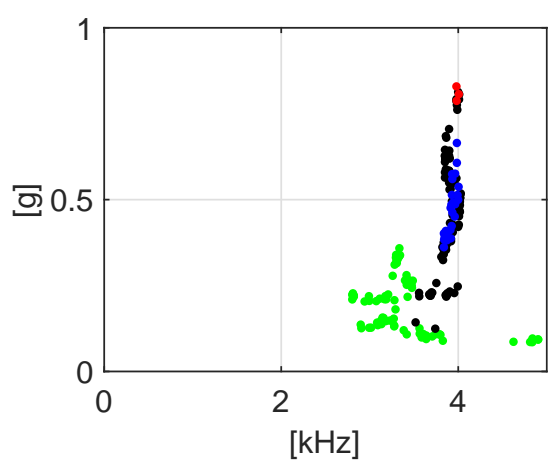

(b) $f_{50}$ versus rms value

Figure 5.16: Classification of the acceleration signal intervals for M6

The low current (green) and non-linear (red) categories are analysed in detail by displaying the extreme values. While for M6 the low current values display the lowest acceleration values, for M10 the acceleration increases up to values comparable to those of the medium and high categories. Furthermore, the high median acceleration detected for the non-linear values at M10 is reflected as a high vibration and low $f_{50}$ value.

The changes on the operational forces are evaluated through a comparison of the TKEO for the main structural resonances between M6 and M10. Figure 5.18 shows four lines representing the average TKEO spectrum for each of the clusters defined previously, displayed in logarithmic scale. Note the overall increment in the vibration values and the change in the harmonic distortion for M10.

The demodulation spectra confirm the electrical nature of the operational forces, as the main peak occurs at $100 \mathrm{~Hz}$. The higher vibration of the low current cluster for M10 is shown as increased harmonic distortion, revealing a distortion of the 


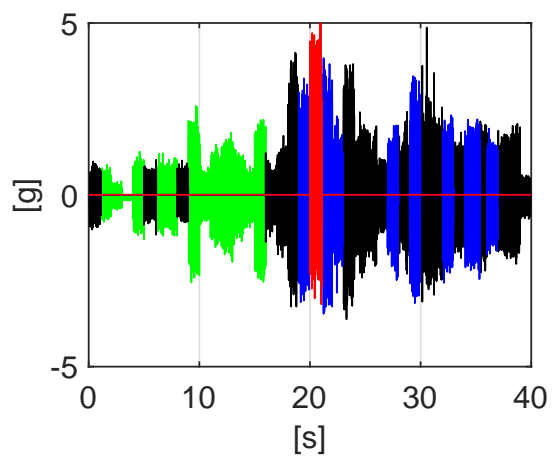

(a) Time waveform

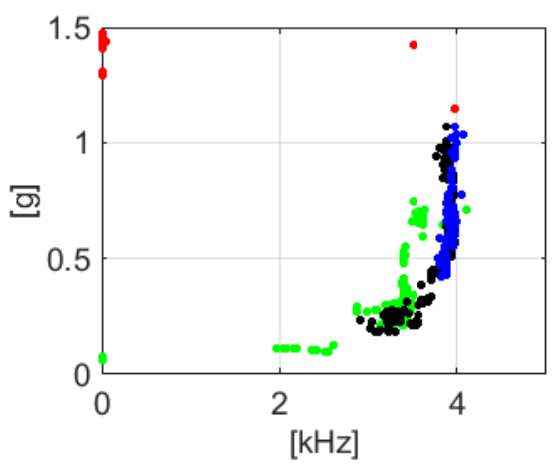

(b) $f_{50}$ versus rms value

Figure 5.17: Classification of the acceleration signal intervals for M10

electrical field. Furthermore, the non-linear cluster at M10 reveals the presence of additional peaks (e.g. $170 \mathrm{~Hz}$ ), the origin of which could not be confirmed.

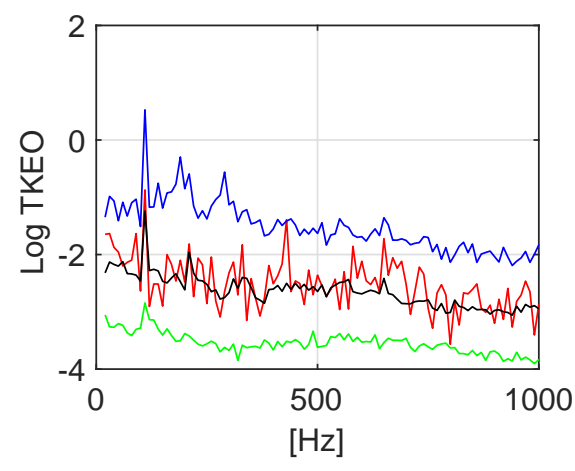

(a) $M 6$

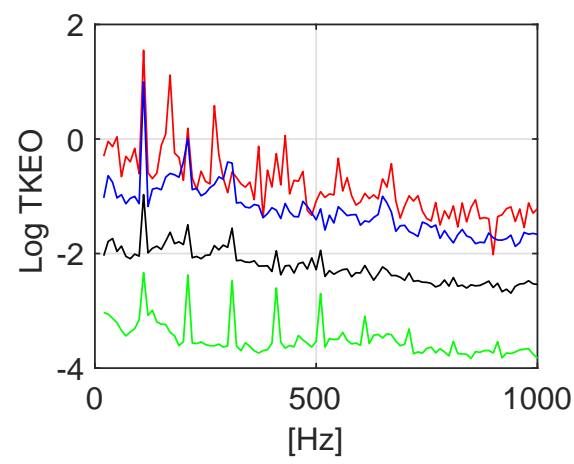

(b) M10

Figure 5.18: TKEO spectra for the main structural resonances for low (green), medium (black), high (red) current and non-linear (red) clusters.

Figure 5.19 presents a comparison of the TKEO spectrum for M6 and M10 at the damage-induced resonances. The figure confirms the harmonic distortion at around $130 \mathrm{~Hz}$, which is more pronounced at M6. For M10 the harmonic distortion is less defined whereas the floor level is significantly higher. The increase in the random vibration is an indication of surface deterioration. 


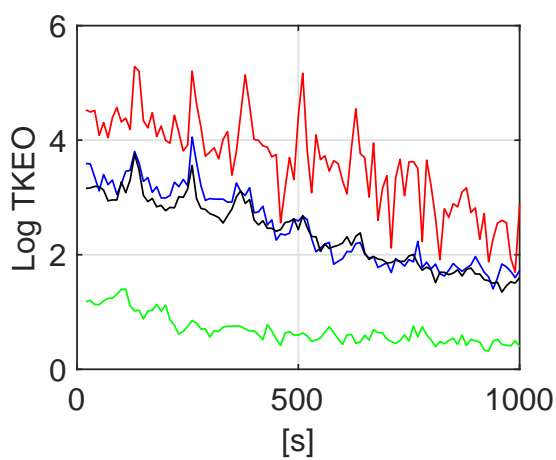

(a) $M 6$

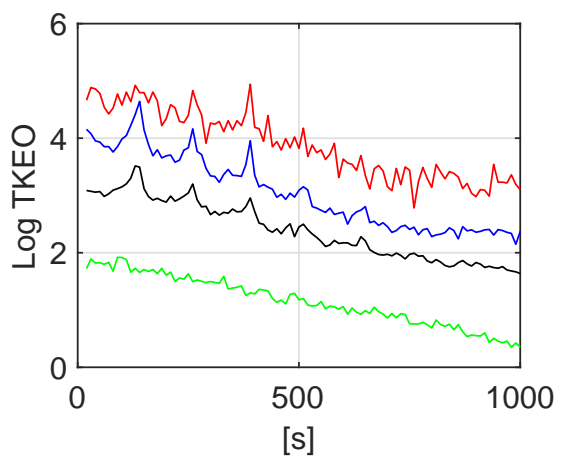

(b) $M 10$

Figure 5.19: TKEO spectra for the damage-induced resonances for low (green), medium (black), high (red) current and non-linear (red) clusters.

In sum, the load distribution as revealed through the vibration response can be described as:

$$
X_{g e n}=H_{s t} \cdot \Delta F_{o p}^{e}+H_{d} \cdot F_{d}
$$

where $\Delta F_{o p}^{e}$ refers to the changes in the electrical forces at the generator. Note that this equation is not entirely applicable to the non-linear states identified at M10, as the additional forces were identified at the demodulation spectrum. It must be stressed that the high variability of the current observed in this case points to additional problems taking place on the system. Hence it is not possible to generalise that the observed vibration behaviour is representative of this type of wind turbine generator.

\subsection{Case III: Train axle box}

The third case addresses the influence of varying environment on the vibration behaviour. This case is developed based on a bearing failure at the axle box of a passenger train (Fig. 5.20). Train axle boxes are the linking subsystem between the rotating wheel set and the quasi-static frame of the bogie [100]. Damage to railway axle box bearings can affect operational efficiency or cause in-service failures, damaging the track and train [101]. 


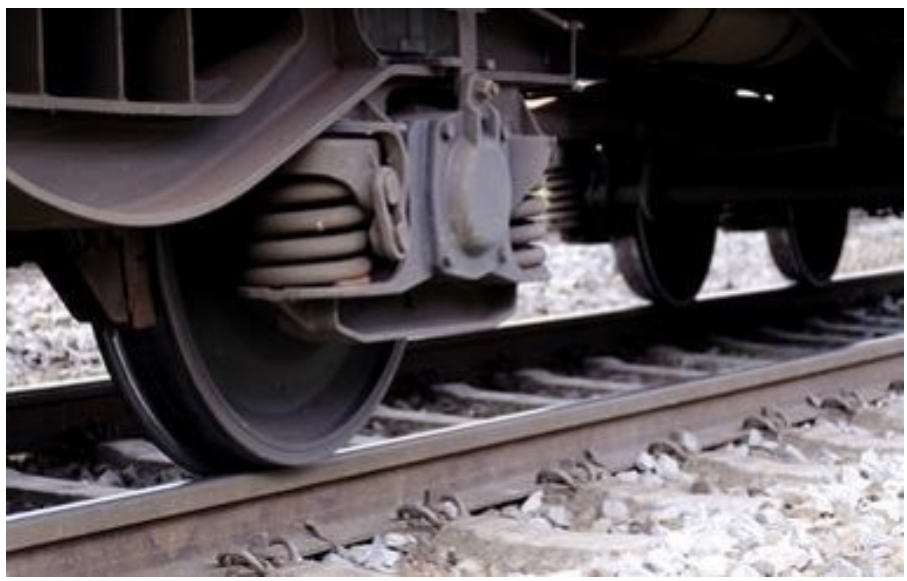

Figure 5.20: Train axle box.

Axle box bearings are exposed to a highly varying environment arising from profile irregularities of a railway track [102]. Such irregularities disrupt the contact forces between the wheel and the rail. As a consequence moderate impacts are transferred to the bearing assembly, which could lead to metal-to-metal contact and excite local resonances, as in the case of damage [86]. Furthermore, the combined effect of track irregularities and bearing damage could lead to dangerous vibration levels risking the axle box integrity [103]. The case developed below exposes the combined effect of damage and track irregularities on the bearing vibration response.

The signals used in this section were captured for bearings $A$ and $C$ located in the same bogie, with bearing $C$ being suspected of damage. Two operational speeds of 5 $\mathrm{m} / \mathrm{s}$ and $13 \mathrm{~m} / \mathrm{s}$ were analysed. The bearings characteristics are shown in Table 5.8.

Table 5.8: Bearing information for train axle box case.

\begin{tabular}{llll}
\hline \multicolumn{4}{c}{ Bearing Information } \\
\hline Sampling Frequency & $25 \mathrm{kHz}$ & Train Speed Low & $5 \mathrm{~m} / \mathrm{s}$ \\
Pitch Diameter & $136 \mathrm{~mm}$ & Train Speed High & $13 \mathrm{~m} / \mathrm{s}$ \\
Roller diameter & $18 \mathrm{~mm}$ & BPFI & $11.9 \times$ \\
Number of rollers & $21 \times 2$ & BPFO & $9,12 \times$ \\
\hline
\end{tabular}




\subsubsection{Signal characterisation}

Table 5.9 presents an overview of the time waveform and spectrogram for the two bearings at the different train speeds.

The time waveforms confirm the variability of the vibration behaviour with respect to the train speed and the instantaneous track irregularities. Such variability is more evident for bearing $A$ at $5 \mathrm{~m} / \mathrm{s}$, for which it is possible to identify discrete peaks and random vibration on top of a constant vibration level. The increment in the train speed has a consequent effect on the magnitude of the vibration levels, both for constant vibration and for the impulsive events.

Bearing $C$ displays significantly higher overall vibration values than bearing $A$. It is also seen that the random vibration behaviour becomes more present at $5 \mathrm{~m} / \mathrm{s}$. At $13 \mathrm{~m} / \mathrm{s}$ the signal displays high vibration values though the behaviour is more homogeneous than at the lower speed. Furthermore, some peak instantaneous vibrations exceeded $20 \mathrm{~g}$, which is significant even for a damaged bearing [103].

\section{Modes identification}

The spectrograms in Table 5.9 reveal two main frequency ranges: the first range attributed to structural vibrations is shown between $0 \mathrm{~Hz}$ and $3.5 \mathrm{kHz}$; the second range attributed to bearing resonances is shown between $6 \mathrm{kHz}$ and $10 \mathrm{kHz}$. Note that the bearing resonance range is significantly higher than for the lab set-up and generator cases.

Bearing A at $5 \mathrm{~m} / \mathrm{s}$ reveals the highest variability in the frequency distribution. The beginning of the sample shows high activity between $6 \mathrm{kHz}$ and $10 \mathrm{kHz}$, yet it reduces towards the end. The comparison with the time waveform shows that such frequency range corresponds to the random vibrations. However, these modes are much less prominent at $13 \mathrm{~m} / \mathrm{s}$, for which the activity focuses below $4 \mathrm{kHz}$. Bearing $\mathrm{C}$ displays a more constant excitation above $6 \mathrm{kHz}$ at the two different speeds.

The spectrograms reveal that the rail track environment causes disruptions to the composition of the modes, as higher frequency ranges can be excited even in the absence of bearing failure. Nevertheless, at higher operating speeds the sensitivity to track disruptions is lower for the undamaged bearing.

A more detailed analysis of the frequency distribution with respect to the local variations in the railway line is presented. Four instantaneous vibration response types are identified: 
Table 5.9: Input signals and spectrograms for the train axle box case.

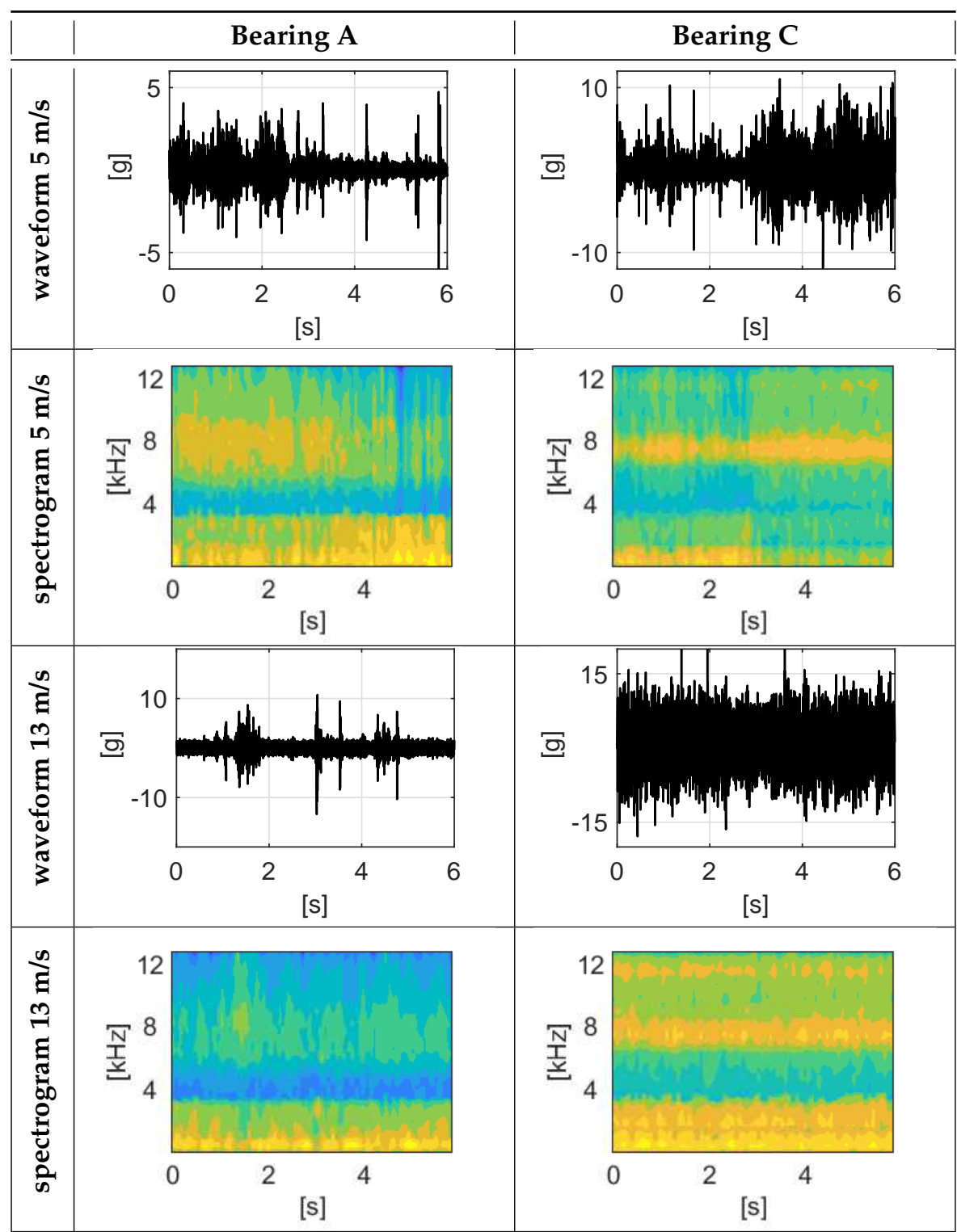


- stable: a constant baseline vibration with the lowest rms and peak amplitude

- impulsive: short time disturbances with high peak-to-peak amplitude

- random: noisy signals of which the duration and amplitude exceed those of impulsive events

- random+impulsive: the combination of impulsive and random excitation.

The details of the classification procedure are presented in Appendix A. Figure 5.21 and Figure 5.22 show the time waveform classified according with the proposed states for bearings $A$ and $C$ at $5 \mathrm{~m} / \mathrm{s}$, respectively. The scatter graph shows the energy distribution evaluation according with the $f_{50}$ feature introduced in the generator case. In both bearings the stable condition (blue) leads to the lower overall and $f_{50}$ values. This condition is used as reference for the assessment of the bearing in the absence of environmental disturbance.

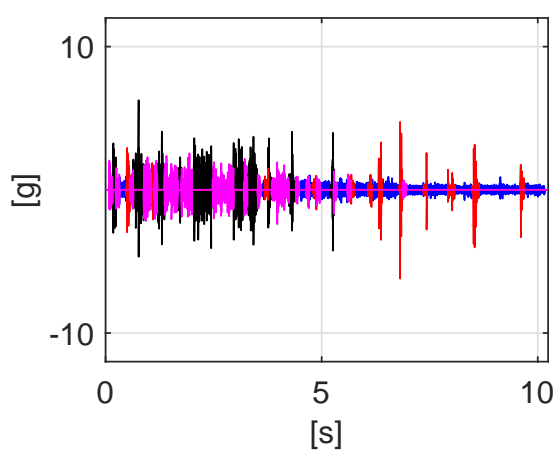

(a) Time waveform.

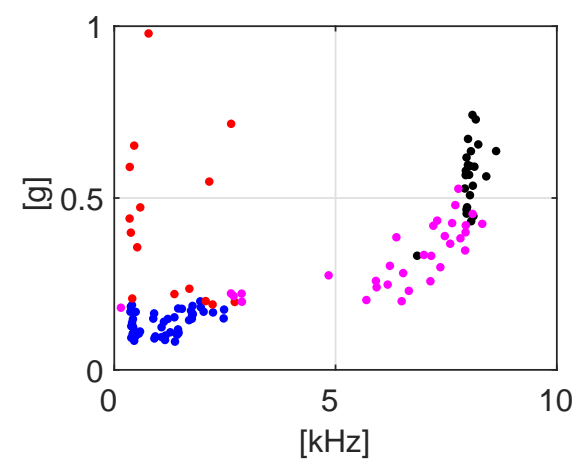

(b) $f_{50}$ vs overall rms value.

Figure 5.21: Classification of instantaneous vibration for bearing $A$ at different railway irregularities: stable condition (blue), impact (red), random (magenta) and random+impact (black) at $5 \mathrm{~m} / \mathrm{s}$

The track irregularities lead to significant frequency distribution for the two bearings. For instance, at bearing A the presence of impacts (red) causes a high overall vibration but excites mainly the lower modes of the system since $f_{50}$ remains at the lower end. The random excitation (magenta) and the superposition of random+impact (black) excite the higher range more predominantly. In contrast to bearing $C$, the separation of these states is less defined as all excitations lead to higher activity of the bearing resonances. 


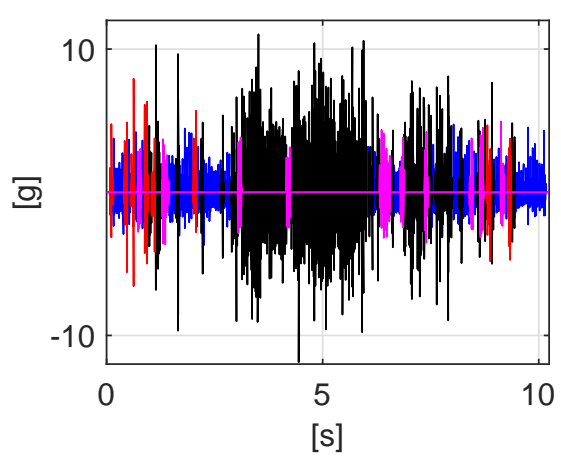

(a) Time waveform.

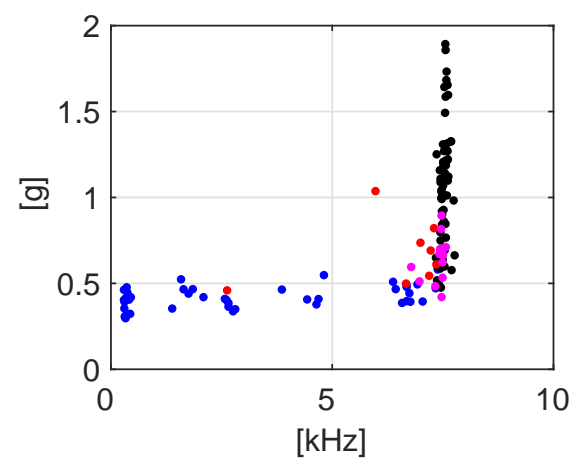

(b) $f_{50}$ vs overall rms value.

Figure 5.22: Classification of instantaneous vibration for bearing $C$ at different railway irregularities: stable condition (blue), impact (red), random (magenta) and random+impact (black) at $5 \mathrm{~m} / \mathrm{s}$.

\section{Force identification}

The identification of the driving force is presented next. Figures 5.23 and 5.24 present segments of at the the stable environment for bearings $A$ and $C$ at $5 \mathrm{~m} / \mathrm{s}$. The time waveform is discussed first. Bearing A shows a random signal with no influence by the rotational forces. Conversely, bearing $\mathrm{C}$ shows repetition of the impulses relating to the bearing damage. The frequency of these impulses is identified at $23 \mathrm{~Hz}$, which is suspected to correspond to the outer bearing fault frequency.

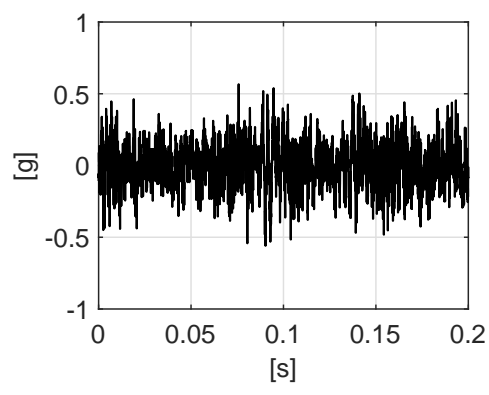

(a) Time waveform.

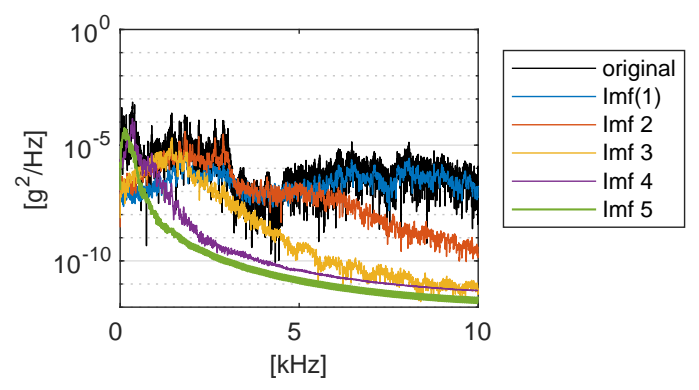

(b) Power spectrum

Figure 5.23: EMD comparison for stable excitation for bearing $A$ at $5 \mathrm{~m} / \mathrm{s}$ 


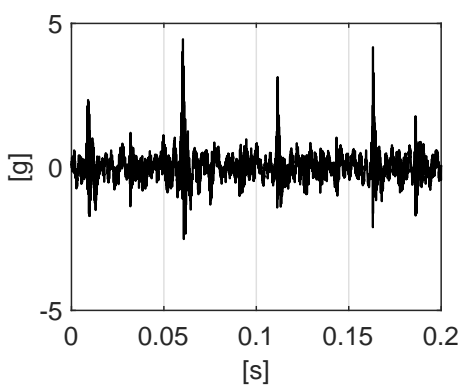

(a) Time waveform.

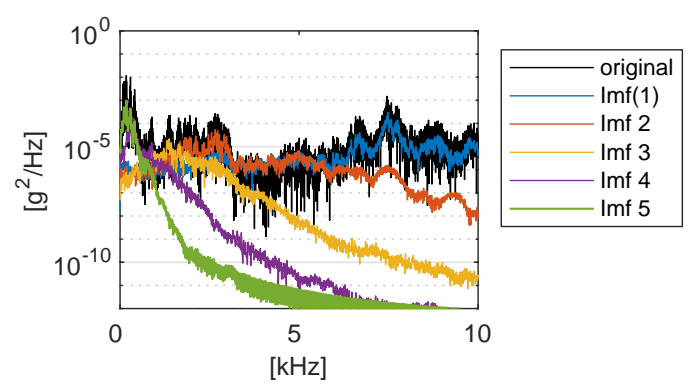

(b) Power spectrum

Figure 5.24: EMD comparison for stable excitation for bearing $C$ at $5 \mathrm{~m} / \mathrm{s}$

The power spectra decomposition is performed using Empirical Mode Decomposition (EMD) [104,105]. This method is used as an adaptive filtering on the signal, which divides the signal into distinct Intrinsic Mode Functions (IMFs) that represent individual modes excited.

In both cases the first IMF relates directly to the bearing resonances (above $6 \mathrm{kHz}$ ). The second appears close to $3 \mathrm{kHz}$ and the third around $1.5 \mathrm{kHz}$. The fourth and fifth modes appear below $500 \mathrm{~Hz}$. The correspondence of each of these modes with the bogie assembly provides significant information about the system's dynamic behaviour, as will be discussed later. It must be noted that the IMF decomposition for bearing A (Figure 5.23b) shows important vibration activity at frequency ranges above $5 \mathrm{kHz}$ even for the stable state. This means that the railway environment always excites the bearing resonances.

A further analysis of the damage-induced forces for bearing $\mathrm{C}$ across the different IMFs is shown in Figure 5.25. The time waveforms and the corresponding TKEO spectra show that the damage-induced forces affect all the identified IMFs. The red marker indicates the identified bearing failure frequency.

In summary, the changes on the track profile lead to a highly unstable vibration response. Yet the analysis of the vibration response shows that:

$H_{s t}$ : In the absence of damage, most of the vibration activity focuses below $3 \mathrm{kHz}$. $F_{o p}^{e n v}$ : No discrete operational forces are identified. Instead, the contact forces arising from track irregularities are reflected as combinations of impulsive and random vibration.

$H_{d}$ : The bearing resonances modes occur above $6 \mathrm{kHz}$.

$F_{d}$ : The damage-induced forces reveal at a frequency of $23 \mathrm{~Hz}$, which excites both the main structural and the bearing resonances. 
IMF 1

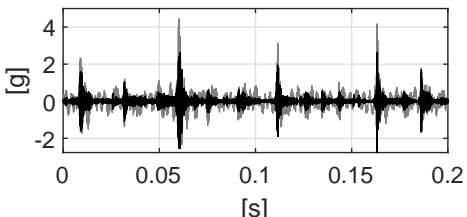

IMF 2

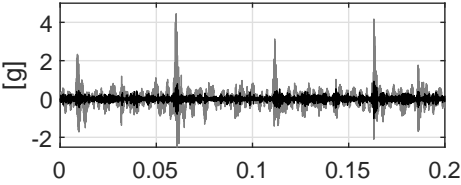

[s]

IMF 3

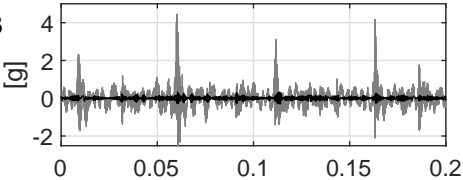

[s]

IMF 4

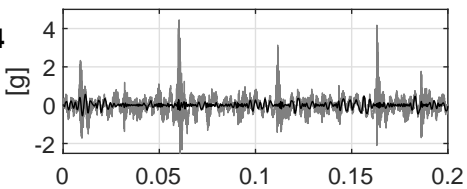

[s]

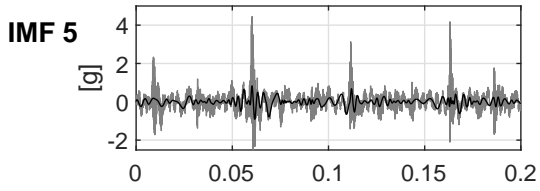

[s]
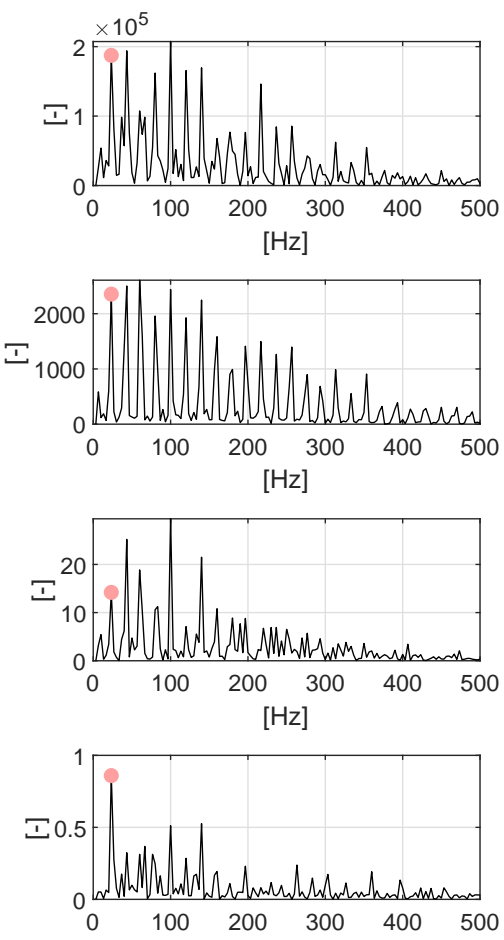

$[\mathrm{Hz}]$

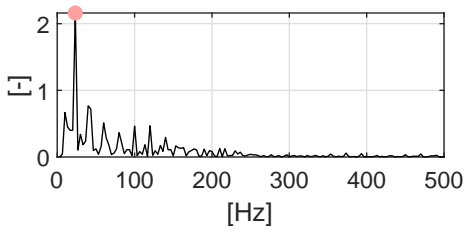

Figure 5.25: IMF time waveform and TKEO spectra for bearing $C$ at $5 \mathrm{~m} / \mathrm{s}$. 


\subsubsection{Functionality evaluation}

The structural support function is defined for the axle box bearing by the capacity of the bearing to provide rigid support despite the track irregularities. As seen, the track instabilities excite the bearing resonances even in the absence of damage. This condition restricts the use of overall $\mathrm{rms}$ values over a fixed frequency range to evaluate the structural support function, as in the lab set-up and generator cases. Instead, the feature $f_{50}$ is used for quantifying the dispersion of the frequency activity.

As introduced in the generator case, the $f_{50}$ feature is defined as the frequency at which the cumulative frequency distribution reaches $50 \%$ of the total energy level. The $f_{50}$ feature supports the evaluation of the function of structural support since a lower frequency means that the system behaves more like a rigid body. A high frequency value, on the other hand, reflects the dominance of higher modes on the entire bogie motion.

Figure 5.26 presents a comparison of the vibration response at different operation speeds for the stable environment. Bearing A shows a stiffening effect, for which the train speed leads to a concentration of the vibration response at the lower frequencies. Conversely, bearing $C$ displays a wider dispersion of $f_{50}$ in addition to a significant increase of the overall values.

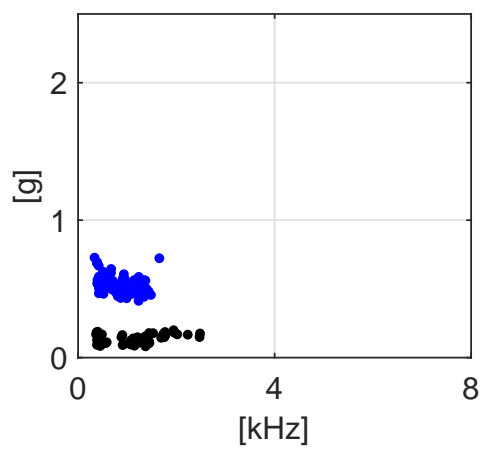

(a) $f_{50}$ vs. rms for Bearing $A$

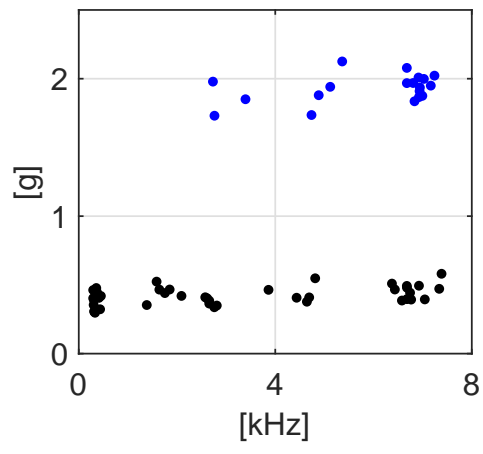

(b) $f_{50}$ vs. rms for Bearing $C$

Figure 5.26: Evaluation support function at stable operation environment for $5 \mathrm{~m} / \mathrm{s}$ (black) and 13 $\mathrm{m} / \mathrm{s}$ (blue).

Similarly, Figure 5.27 presents a comparison for the random operational environment. This figure highlights even more the stiffening effect for bearing A, while for bearing $\mathrm{C}$ the vibration response is dominated by the local resonances. 


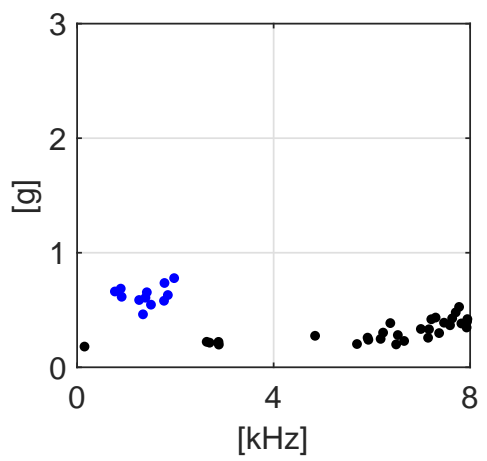

(a) $f_{50}$ vs. rms for Bearing $A$

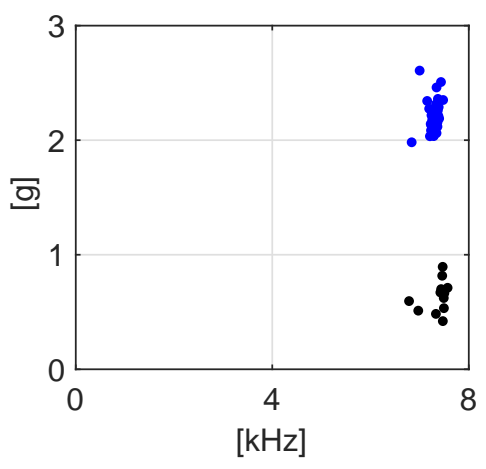

(b) $f_{50}$ vs. rms for Bearing $C$

Figure 5.27: Evaluation support function at random operation environment for $5 \mathrm{~m} / \mathrm{s}$ (black) and 13 $\mathrm{m} / \mathrm{s}$ (blue).

\subsubsection{Load distribution evaluation}

The analysis of the load distribution for all the identified states is performed based on the EMD analysis, similarly to the stable case in Figure 5.24. Figure 5.28 shows the distribution five distinctive IMFs for the bearing $C$ at $5 \mathrm{~m} / \mathrm{s}$. The scatter graphs show the highest frequency and the corresponding rms value for each IMF.

The first IMF is consistently displaying a peak frequency at $8 \mathrm{kHz}$. It also displays the highest rms values for the stable, impulsive and random states. For the random+impulsive case, it is seen that the IMF 5 (lowest frequency) displays also comparable acceleration values. The higher IMFs (lower peak frequencies) also display high consistency. All of them appear below $4 \mathrm{kHz}$.

IMF 5 (lowest peak frequencies) shows the increment of the vibration response at the for the random and random+impulsive states, up to $4 \mathrm{~g}^{\prime} \mathrm{s}$ in the later case. In a similar way to the events of nonlinear state in the wind turbine generator case, the occurrence of such events implies a high risk for the system stability.

In sum, the derived load distribution for the train axle box case is:

$$
X_{\text {train }}=\left(H_{s t}+H_{d}\right) \cdot \Delta F_{o p}^{e n v}+\left(H_{s t}+H_{d}\right) \cdot F_{d}
$$

where $\Delta F_{o p}^{e n v}$ refers to the varying contact forces due to the wheel-rail interaction. This expression shows a similarity to the lab set-up case as both structural resonances and damage-induced resonances are affected by the operational and damageinduced forces. 


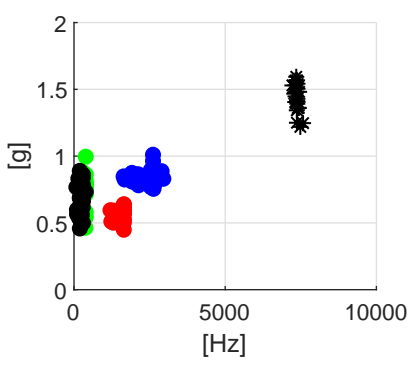

(a) Stable.

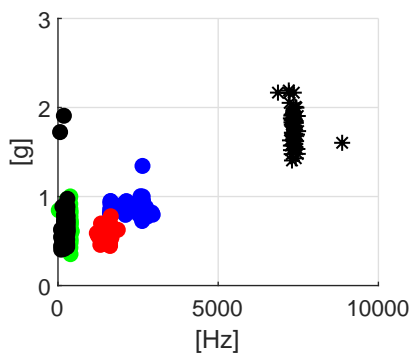

(c) Random

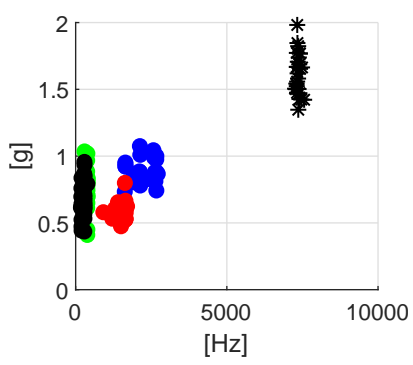

(b) Impulsive

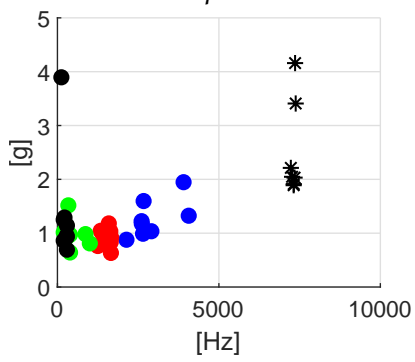

(d) Random+Impulsive

Figure 5.28: EMD comparison for stable excitation for bearing $C$ at $5 \mathrm{~m} / \mathrm{s}: \operatorname{IMF} 1\left(^{*}\right), \operatorname{IMF} 2$ (blue), IMF 3 (red), IMF 4 (green), IMF 5 (black).

\subsection{Discussion}

The cases of roller bearing failure have been developed to illustrate how to reduce the uncertainty of the failure behaviour. This characterisation included the major load distribution changes arising from bearing damage, and the within-state variation in the load distribution changes according with the kinetic energy principle. The main findings with respect of the goals formulated for the chapter are given below.

\section{Component evaluation}

The evaluation of bearing failure has been addressed in relation to the structural support function. The proposed evaluation is based on generic evaluation of the component role and a further refinement based on the system-specific operational context. Two generic features were proposed on the basis of the energy distribution displayed. The first feature type was based on the rms vibration at the main structural resonances of the system. This was applied to the lab set-up and the generator 
bearing evaluation, as for these cases the operational forces appeared concentrated at the structural resonances. However, the train case criteria were shown to be inappropriate given the broader effect of the environment forces throughout global and local modes. An alternative feature type was defined based on the energy distribution across the entire frequency range analysed.

The refinement of the bearing evaluation feature was performed according with the wind turbine generator and train axle box operational environments. For the generator case this implied that the structural support function was evaluated with respect to the output current, and for the train case the energy distribution was evaluated with respect to expected stress stiffening due to increasing speed. These examples illustrate the novel use of vibration features to evaluate the impact on system level aspects due to bearing damage.

\section{Load distribution evaluation:}

The vibration signals were analysed so as to characterise the major changes in load distribution. This analysis is based on the identification of the forces and modes responsible for the vibration response and the interactions between them, as shown in Table 5.10 .

The lab set-up and train cases allowed the evaluation of the normal vibration behaviour in the absence of damage. This showed that in the lab set-up the modes were excited mainly by discrete residual imbalance forces. The train case showed the effect of broad-band excitation, for which the local bearing resonances were excited even in the absence of damage.

The three cases show significant differences with respect to the modified dynamic behaviour, comprising the changes in the participation of the operational forces and the structural resonances. The operational forces for the lab set-up and generator cases were of a discrete, mechanical and electrical nature. However, the train case reveals a more diverse range of normal system excitations due to the distinct changes in the track profile. This differentiation between discrete, random and impulsive response types has a direct effect on how the structural resonances are excited and therefore explains the different approaches on bearing functionality evaluation, as discussed before.

The damage-induced vibration displayed a more homogeneous behaviour in comparison with the modified dynamic behaviour. In all cases the damage-induced resonances were identified at a frequency range higher than the main structural resonances. The variations in the specific frequency could be attributed to the different bearing types analysed and their dimensions. Also in all the cases the forces displayed a repetitive behaviour which could be identified at the demodulation spectra. 


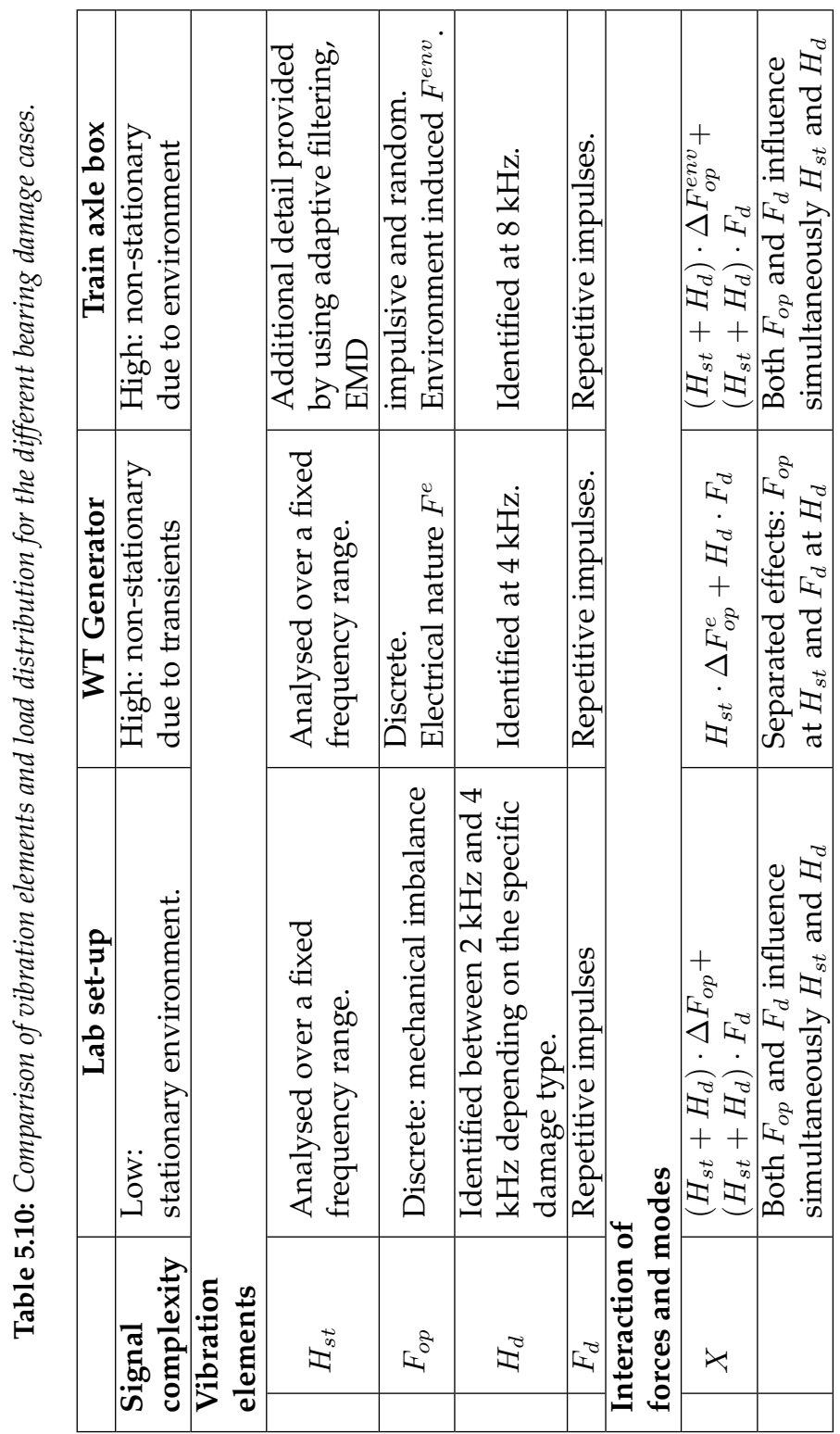


The interaction between the driving forces and excited modes is reflected in distinctive functions $X$ that represent the load distributions associated with each particular system. In the generator case the operational forces were revealed only at the range of structural resonances, and the damaged-induced forces at the corresponding damage-induced resonances. Conversely, the lab set-up and the train cases revealed a more mixed situation, where the damage-induced forces propagated across all the ranges identified, and the operational forces excited the bearing local resonances in addition to the structural resonances.

\section{Failure state variations:}

The last objective concerns the variations within the major load distribution changes. As postulated by the kinetic energy principle, quantitative variations of the failure behaviour within specific states depend on the kinetic energy associated with the impaired functions, the physical characteristics of damage, and the operational environment.

As discussed previously, the specifics of the structural support function with respect to the system context are reflected in the different approaches to component evaluation. Furthermore, the influence of the physical characteristics of damage was shown for the lab set-up. The comparison between the different damage types showed a similar response for the inner and outer race cases. However, despite having the same physical characteristics, the inner race damage had the most severe effect on the structural support function and the free rotation functions. This could be due respectively to the proximity to the moving frame and the highest speed.

Furthermore, the generator case showed the effect of advanced damage degradation. Thus, while for the first analysed period M6 the variation with respect to current seemed to be mainly quantitative, for the more advanced state at M10 different type of abnormalities emerged. These included an exacerbated harmonic disruption of the electrical forces at low current levels and critical outliers.

The influence of a varying operational environment was shown in the generator and train cases, though the two cases revealed very different effects. For the generator case the variations were identified mainly for the magnitude of the driving forces. The railway environment was shown to be more complex, given the random and impulsive nature of the contact forces.

Despite these variations, the load distribution could be represented mostly by distinctive functions. However, a few exceptions were found that included qualitative changes. In the lab set-up case the roller element damage showed different effects on the operational forces than the inner and outer race damage. This is explained by the fact that the damage-induced forces at the roller elements had sufficient energy to 
proliferate beyond the corresponding shaft. Moreover, the generator and train cases displayed some outliers of high vibration values and distinct frequency composition, particularly at the nonlinear state at M10 in the generator case and random+impulse state at the highest speed in the train case. These exceptions provide indications of emerging, unstable failure states.

\subsection{Conclusion}

The investigation of roller bearing failure illustrates the increased uncertainty in the system behaviour as a result of the effect of failure. As stated in the kinetic energy principle, uncertainty develops as the kinetic energy interacts in unanticipated ways with the system, leading to further destabilisation. This manifests itself in the emergence of damage-induced forces and the excitation of local resonances, the changes in the operational forces and how the structural resonances become excited, and the exacerbated variations in the system response against operational environment changes.

The comprehensive vibration analysis developed in this chapter is proposed as guidance on how to characterise failure behaviour. First at all, it was shown that although the investigated failure mode originated at the component level, the resulting response must be evaluated considering the system functional context. In other words, the overall system functionality, as well as the component's role. Additionally, it was shown how to characterise the variation of the load distribution for varying operational environments. This contributed to an enriched understanding of the conditions that lead to the most significant changes on the vibration response. In sum, the functional approach to vibration analysis led to a quantitative model of the changes in the dynamic behaviour associated with a failure state. 


\section{Chapter 6}

\section{Complexity: Rotor-blade systems case}

This chapter discusses the last aspect of failure behaviour: the increased complexity caused by failure-induced functional interdependencies. Complexity was introduced in Chapter 1 as the property of a system that makes it difficult to understand partly due to the interdependencies exhibited among the components. It was also pointed out that dependencies and interactions cannot be regarded only as a negative feature since achieving an overall advantageous functionality is often possible only by the high synergistic interdependencies enforced by design.

In Chapter 2, the designed complexity was attributed to the functional and structural interdependencies (Figure 2.3). It was also emphasised that not all possible interdependencies can be effectively identified at design. This explains why for some components their constitutive material's actual capacity turns lower than expected, as in the case of simple failures.

For the case of complex failures, a new type of dependency was identified. This refers to the increased influence of the material deterioration on the functionality degradation, leading to profound changes in the system load distribution (i.e. nonlinear system behaviour). However, this type of dependencies are not expected on a system following the designed ageing. Instead, they are conditioned to an already degradation process. This means that they increased dependency between material and functional degradation is responsible of the proliferation of failure, as seen in the complex case. 
The effect of increased functional dependencies on failure proliferation was analysed in Chapter 3. Specifically, the extent that a perturbation (i.e. new failure mode) exacerbates the ongoing degradation for a particular set of functions, so that the combined effect is a reorganisation of the load distribution (i.e. new failure state). Thus, the likelihood of a new failure mode depends on the coincidence of functions affected by the incoming failure mode and existing base failure as stated by the mode-state dependency principle:

Mode-State Dependency Principle The likelihood of an incoming failure mode (perturbation) triggering a new failure state increases with the actual system complexity.

The increased system complexity reflects as the difficulty to understand how one of the effects of failure is that the functions become increasingly interdependent. Yet the analysis of the vibration behaviour supports the observation of the failure behaviour. As discussed in Chapter 4 the new interdependencies manifest themselves as new nonlinear interactions between the driving forces and excited resonances, both of operational and damage origins.

The present chapter illustrates the use of vibration analysis for characterizing the increased complexity due to failure. This is developed in an experimental study of a rotor blade demonstrator. The demonstrator is designed to allow different combinations of perturbations in a system with controlled designed dependencies. The experimental study is conducted to (i) illustrate the increased nonlinear response for advancing failure, and (ii) assess the dependency between failure modes to trigger new emerging failure states.

The analysis of the failure behaviour is based on the response of a semi-clamped beam to different perturbations. The response is analysed through a combined approach for the spatial and temporal assessment of the vibration behaviour as discussed in Section 4.3. Unlike spatial-only type of assessments that focus on the damage characteristics (i.e. modal analysis and vibration-based SHM), the proposed combination of temporal and spatial assessment enables the functional evaluation of the test beam in relation to the operational context.

The chapter is organised as follows. Section 6.1 introduces the main characteristics of the demonstrator based on a synthesis of helicopter the rotor blades. Section 6.2 explains the experimental design for inducing different failure behaviour. The evaluation methods of the blade response are presented in Section 6.3. Section 6.4 discusses the results for the individual failure modes and their combinations. The general conclusions of the case and what it means for the failure assessment of systems are presented in Section 6.6. 


\subsection{Rotor blade demonstrator}

The set-up used for the study of failure proliferation is based on rotor blade systems. Rotor-blade systems refers to the sub-assembly of rotating mechanical systems where the power transformation between the (fluid) medium and the mechanical shaft occurs through the aerodynamic effect of a rotating blade. Wind turbines and helicopters rotors are prototypical examples of these. The assembly comprises the rotating blades, the hub which enables different degrees of freedom of the blade, and the control instruments.

The study of failure behaviour on rotor blade systems is motivated by the fact that despite their relevance to the performance and reliability of rotating mechanical systems, their failure behaviour is poorly monitored due to restricted access to the rotating blades. Thus, current assessment is based on the transmitted vibrations to the drive train and other structural components (e.g. helicopter fuselage or wind turbine tower); however, the transmission path adds significant disturbances to the signal which hinders the interpretation of the blade response. For the experimental study the test beam representing the rotating blade response is monitored directly. The results derived from this study will motivate the development of on-blade sensing, as will be discussed in Chapter 7. This section describes the test set-up based on distinctive functional and behaviour aspects of rotor blade systems.

\subsubsection{Rotor-blade systems}

The requirements for the rotor blade demonstrator are defined on the basis of the synthesis of a helicopter rotor system following the Function-Behaviour-Structure. As introduced in Section 1.5.1, the FBS method is used to explain the designed complexity for the context of failure. Specifically the articulated rotor type for the operational states of hover and forward flight was analysed [106].

Rotor blade (sub)systems correspond to a particular type of rotating structure that enables the exchange of kinetic energy between a fluid medium and a mechanical shaft. The medium can be the source of power as in the case of wind turbines, or the recipient as in helicopters. These refer to rotary systems whose blades have independent motion with respect to the rotor angular motion. Such rotation can be feathering of the blade, flapping and lagging, as shown in Figure 6.1.

From these elements, the power transference takes place at the rotating blades, for which three main functions were identified: (i) to maximise lift by enforcing lower flapping modes, (ii) to minimise drag by restricting in-plane vibration and (iii) to minimise tip instabilities as these increase drag. 


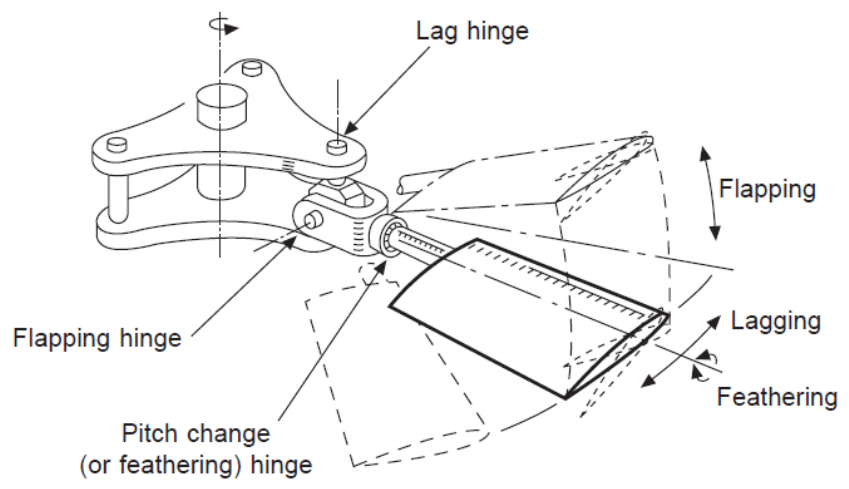

Figure 6.1: Articulated rotor blade systems ${ }^{1}$

These three functions are not independent as the dynamics of rotating blades impose dependencies between the vibration modes supporting the functions. For instance, rotating blades exhibit coupling between lagging and flapping motion as a consequence of the Coriolis effect, the torsional elastic modes and the blade geometry. This coupling creates a dependency between lift and drag. Additionally, the participation of rigid and elastic flapping modes is defined by the rotor type (i.e. semi-articulated, rigid), stress stiffening effect and helicopter weight. This relation creates an interdependency between lift and tip stability.

Furthermore, the three functions rely on common material properties defining the stiffness, damping and mass distribution of the blade. For instance, the consumption of material fatigue life is reflected in the stiffness degradation on which all the functions depend $[108,109]$. This dependency between functional and performance parameters corresponds to the expected blade ageing (see Section 2.2.1). Furthermore, unexpected events can exacerbate the dependency between the material consumption and the blade functional deterioration, enforcing a subsequent change in the drive train load distribution. For example, the mass imbalance resulting from moisture absorption.

Alternatively, the power transference can be affected by damages originating from components of the sub-system other from the blades, such as problems with the control affecting the force input, and lag damper issues leading to high in-plane motion. These problems affect not only the blade functionality but also the transmitted (torsional) vibrations to the drive train. An overview of these main findings regarding the helicopter rotor is shown in Table 6.1.

\footnotetext{
${ }^{1}$ Taken from Bramwell et al [107]
} 
Table 6.1: FBS decomposition for helicopter main rotor blades.

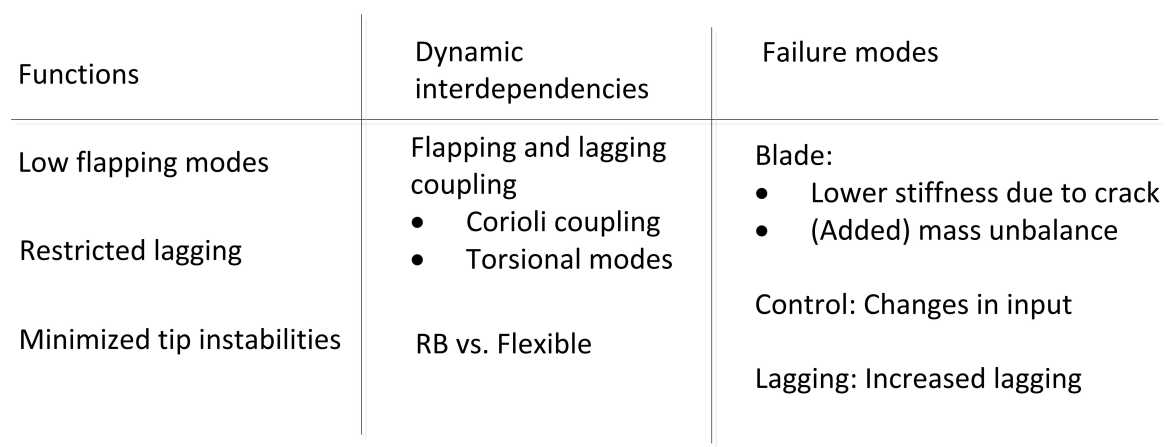

\subsubsection{Demonstrator}

The presented synthesis of a helicopter rotor blade is taken as a reference for the demonstrator requirements. The set-up was designed to represent the coupling between lagging and flapping, the effect of boundary stiffness for the ratio between rigid motion and elastic flapping modes, and the effect of changes in the input loads. For safety reasons no rotating parts were allowed for the experiments, hence the dynamic aspects are simulated in a nonrotating test beam. The design, construction and validation of the demonstrator are reported in separate references $[110,111]$.

Figure 6.2 shows a schematic of the set-up, which comprises a test beam supported by two hinges that provide independent support of the flapping and lagging direction. The beam is semi-clamped, enabling different participation or rigid body and elastic modes. The values of the torsional spring stiffness are selected to represent the ratios between rigid body and elastic modes, and between rigid flapping and lagging modes of an actual helicopter blade [112].

The input is delivered by two shakers for the flapping and input direction. Additionally, two load sensors are used as feedback of the excitation. Finally, the fixture is supported by a fixed frame (not shown). The main characteristics based on the dynamic phenomena and failure modes are highlighted below.

Flapping and lagging: The test beam is supported by two hinges that enable the independent excitation in the flapping and lagging direction. Each hinge is constrained by a torsional spring that regulates the boundary stiffness at the beam base.

Rigid and flexible flapping: The beam is semi-clamped. Different participation of rigid flapping motion is achieved by thee sets of torsional springs. 

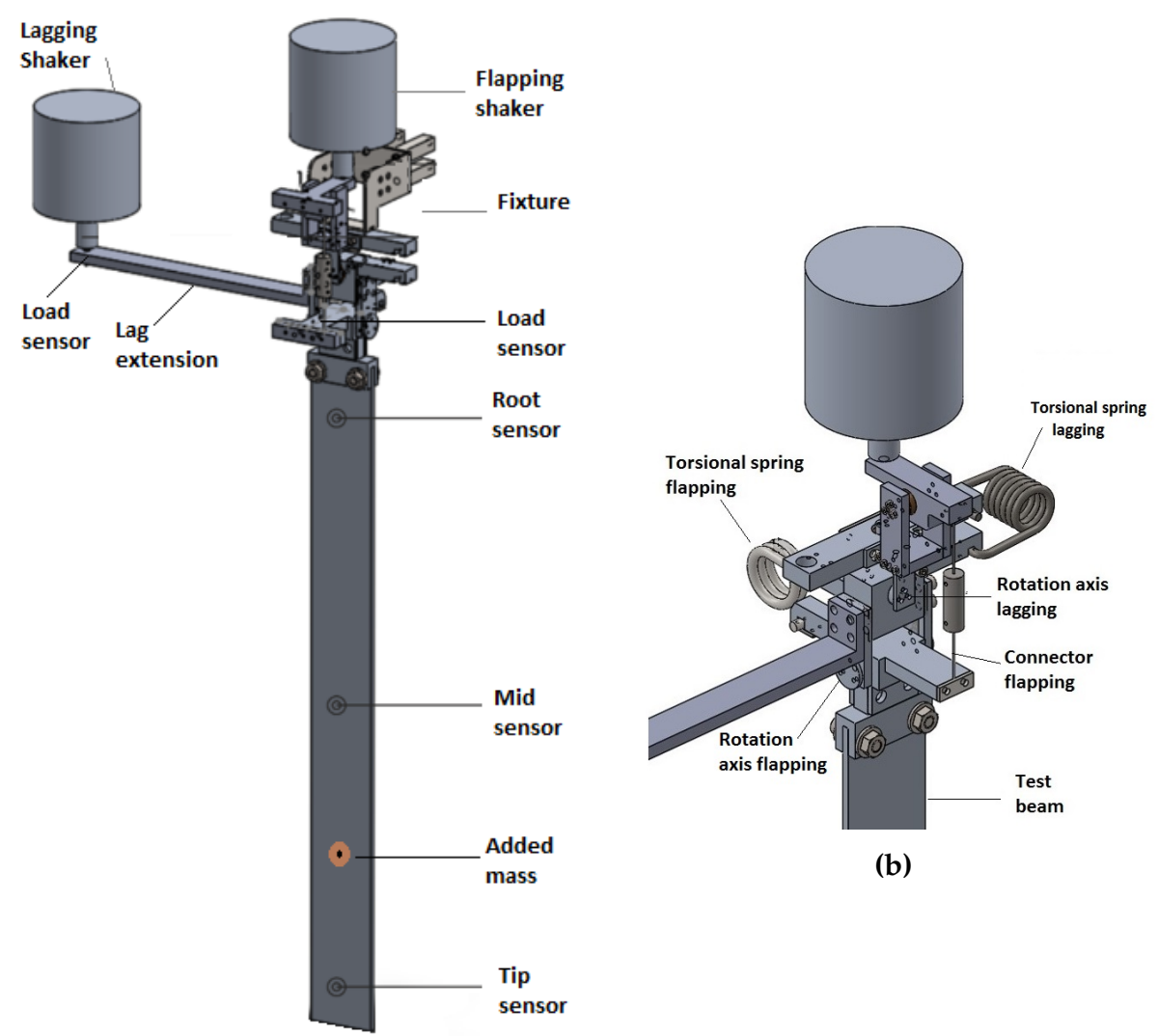

(b)

(a)

Figure 6.2: Demonstrator of rotor blade systems.

Input: The flapping and lagging inputs correspond to a sinusoidal excitation $\Omega$ at $15 \mathrm{~Hz}$, representing an axi-symmetric load. Additionally, the sinusoidal input can be distorted by adding harmonic (multiples) of $\Omega$. Two force sensors were placed at the top part of the generator to monitor the system feedback.

On-blade sensing: Three acceleration sensors are located on the blade for registering vibration at the root, mid-section and tip section. 


\subsection{Experimental design}

As introduced before, the experimental study intends to illustrate the increased nonlinearity due to emerging failure states and the dependency between failure modes and the base failure states that lead to new failure states. For the demonstrator, the beam performance is evaluated through the participation of the three flapping modes in the total response:

- Rigid body (RB)

- First flapping mode (1FE) and

- Second flapping mode (2FE).

The following subsections describe the test variables representing different failure modes of rotor blade systems and the evaluation parameters.

\subsubsection{Test variables}

For the demonstrator, the nonlinearity will be assessed through the (changes) in the harmonic response to a given input excitation and also under different failure modes, as presented below.

Reference [Ref] The reference condition is defined for the test beam when excited at a forced sinusoidal excitation in the flapping direction. The boundary stiffness is prescribed with a torsional spring of $112 \mathrm{Nm} / \mathrm{rad}$ torsional stiffness, which guarantees a ratio between the rigid body and first elastic blade similar to a conventional helicopter. The time waveform of the tip, as shown in Figure 6.3, reveals a slight deformation at the positive extreme of the sinusoidal (i.e. close to +1 ). This disruption is shown at the FFT spectrum by the presence of the second and third harmonic. This indicates an intrinsic nonlinearity at the demonstrator most likely due to the beam's flexibility and the residual play at the lagging hinge.

Two types of variations from the reference condition are introduced. The first type corresponds to the structural variation of the dynamics properties. These are the variations in the boundary stiffness by the softer torsional springs, and mass distribution by a point mass that mimics a case of damage.

Variable 1. Torsional springs [TS1,TS2] The change in boundary stiffness is enforced through two softer torsional springs for the lagging hinge. These conditions 

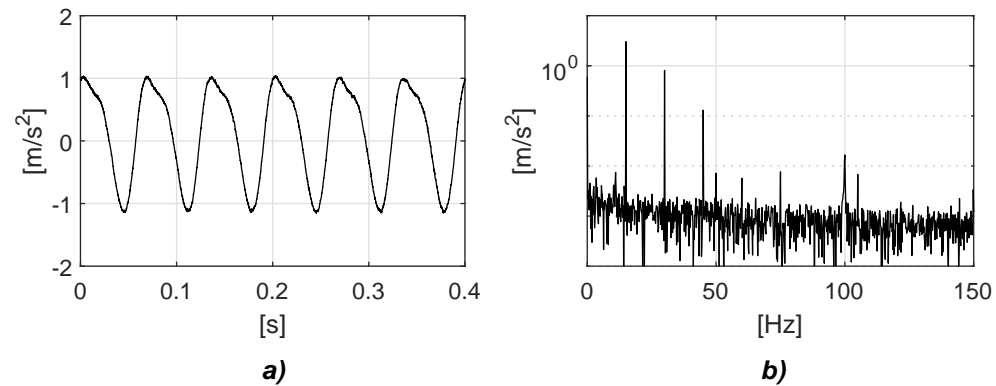

Figure 6.3: Reference condition HP: a) time waveform, b) spectrum.

are labelled as $B S 1$, corresponding to a medium spring ( $80 \%$ of the reference value), and $B S 2$, corresponding to the softer spring ( $62 \%$ of the reference value).

Variable 2. Added Mass [-AM] The change in the mass distribution is enforced by an added point mass located at the one quarter of the beam length from the beam tip. The weight of the added mass is 15 grams, which corresponds to $7 \%$ of the total beam mass. The dash symbol preceding the case abbreviation ( - ) represents no additional variations in the input force.

In addition to the changes in the structure, two more variables corresponding to the excitation variations are introduced. These relate to changes in the lagging and flapping inputs.

Variable 3. Lagging input $[+\mathbf{L 1},+\mathbf{L 2}]$ The third variable corresponds to the excitation in the lagging direction simultaneously to (reference) flapping excitation. The frequency of the lagging excitation is also $15 \mathrm{~Hz}$ as in the flapping excitation. Two levels of excitation amplitude are used: a lower level $L 1$, corresponding to $20 \%$ of the flapping excitation, and a higher level $L 2$, corresponding to $40 \%$ of the flapping excitation. The disruptions in the flapping response due to the lagging excitation indicate coupling between the two motions. Such coupling is explained both by the kinematics of the demonstrator and by the excitation of torsional modes of the test beam.

Variable 4. Harmonic Distortion [+HD1, +HD2] The last variable is the harmonic distortion of the input flapping excitation. This case is based on the principle of Higher-Harmonic Control (HHC) for the reduction of the lift at selected points of the 
rotor circumference [113]. The truncation of the sinusoidal wave is achieved by the selective excitation of higher modes at odd orders of the base frequency. Two levels of harmonic distortion were examined. The first level $H D 1$ considers an additional excitation at $45 \mathrm{~Hz}$, while the second level $H D 2$ includes an additional excitation at $75 \mathrm{~Hz}$. The amplitude of the distortion at $45 \mathrm{~Hz}$ is limited to $10 \%$ of the amplitude of the reference excitation to limit the excitation of the first flapping mode of the beam. The amplitude of $75 \%$ is set at approximately $5 \%$ of the base excitation.

The vibration response resulting from these test cases will be analysed under the different performance parameters. Also it will be investigated whether the increased severity for the variations in the torsional string, lagging input and harmonic distortion (i.e. $T S 2, L 2$ and $H D 2$ ) lead to qualitative differences in the vibration response with respect to the lower severity levels.

\subsubsection{Procedure}

As introduced in Section 3.4 failure drifts the system behaviour away from the designed state through failure states, where each failure state corresponds to a particular reorganisation of the system load distribution. The difference between a failure state and a failure mode is that the former refers to the way in which the system is currently operating, while the latter refers to the way in which the operation of the system is changed. This means that a failure state can be affected by multiple failure modes, but also that the addition of a new failure mode does not necessarily trigger a new reorganisation of the system.

Figure 6.4 illustrates the effect of failure mode(s) on achieving a new failure state according with the three functional parameters defined for the demonstrator. The Figure shows an inner triangle that represents intrinsic nonlinear mode of operation as seen for the reference condition and the dashed line represents the transition to a new failure state. The red line shows the effect of one or multiple failure modes on the deviation from the performance parameters defined for the test beam. In this example the rigid body behaviour is the parameter that is most affected by failure.

The experiments are conducted to characterise the transition to new failure states as a consequence a new failure mode (perturbation). This is stated as (Eq. 3.2)

$$
T_{i+1}=f\left(T_{i}, \theta_{i+1}\right)
$$

where the emergence of a new failure state $\Theta_{i+1}$ reflects the combined effect of the base failure state $\Theta_{i}$ and a new additional perturbation or failure mode $\theta_{i}$. The proposed failure mode for the demonstrator is the harmonic distortion $\theta_{H D}$. 


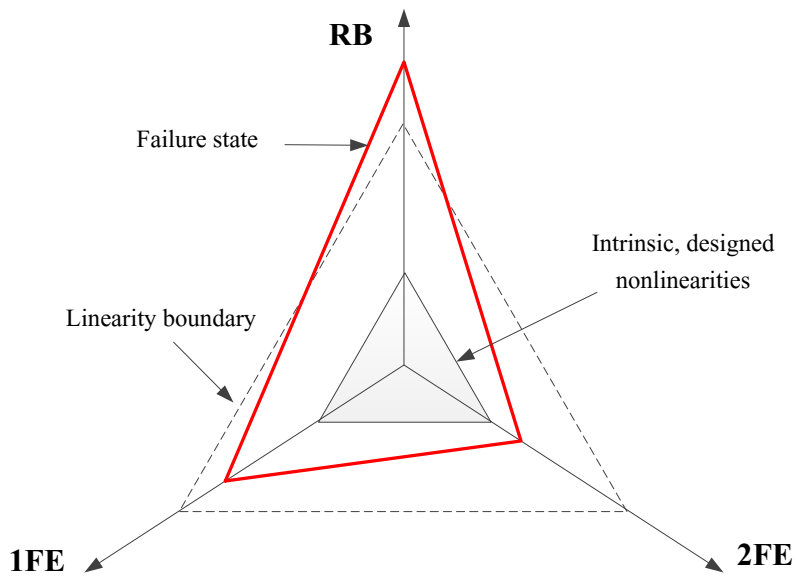

Figure 6.4: Failure behaviour of demonstrator under failure.

The base failure states combinations are defined as shown in Figure 6.5. Two types of base failure state $\Theta_{i}$ are considered. The first set comprises structural variations of the torsional spring for the flapping hinge, and the added mass. The second set includes the lagging excitation.

\begin{tabular}{|c|c|c|}
\hline$\stackrel{\dot{\Phi}}{\check{\Phi}}$ & Reference condition & (Ref) \\
\hline \multirow{2}{*}{ 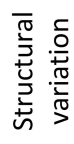 } & Torsional spring & $-\mathrm{TS} 1,-\mathrm{TS} 2$ \\
\hline & Added mass & $-A M$ \\
\hline \multirow{2}{*}{ 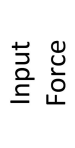 } & Lagging excitation & $+\mathrm{L} 1, \mathrm{~L} 2$ \\
\hline & Harmonic distortion & $+\mathrm{HD} 1,+\mathrm{HD} 2$ \\
\hline
\end{tabular}

Figure 6.5: Overview of test cases 


\subsection{Vibration response analysis}

The response of the beam to the different test conditions is analysed to identify and explain the nonlinearities that characterise each failure state. Three methods are used for the evaluation of the vibration response. Firstly, the decrease in linearity is assessed through the response at the excitation frequency. Secondly, the increased excitation of the first flapping mode is analysed through the phase portraits of the tip response. And finally, the verification of the nonlinearity assessment is carried through the participation factors of the flapping modes.

\subsubsection{Response at excitation frequency}

The most immediate effect of changes in the system linearity/nonlinearity is the reduction in the system output with respect to the input (Eq. 2.4). For the demonstrator the first indicator of system nonlinearity is the response of the test beam at the excitation frequency $X_{1 \Omega}$. Reductions of amplitude at $X_{1 \Omega}$ reflect the degree to which the energy is transferred to higher frequencies. This is seen at the reference case (Figure 6.3) for which the tip response reveals the presence of the second and third harmonics even though these are not excited.

Variations of the linear response can also be expected by changes in the input excitation as in the lagging and harmonic distortion cases. For these it is possible that part of the input energy is reflected at $X_{1 \Omega}$, even though the energy is added to the system at a different direction or frequency respectively.

\subsubsection{Phase portrait}

A more detailed assessment of the nonlinearity is conducted using phase portraits, as introduced in Chapter 4 . For the beam case the state variables correspond to the measured acceleration and the calculated velocity. The phase portraits are used to analyse the participation of the third harmonic $X_{3 \Omega}$ on the tip response. This order is critical as it coincides with the first flapping mode of the system. The tip response $X$ is approximated by

$$
X=X_{1 \Omega} \sin (2 \pi \Omega t)+X_{3 \Omega}(2 \pi(3 \Omega t+\phi))
$$

where $\Omega$ corresponds to the excitation frequency $(15 \mathrm{~Hz}), X_{1 \Omega}$ to the response at the input excitation, and $X_{3 \Omega}$ to the distortion at the third harmonic $3 \Omega$ and the corresponding phase delay $\phi$. Figure 6.6 illustrates a comparison of different magnitude and phase of $X_{3 \Omega}$. 

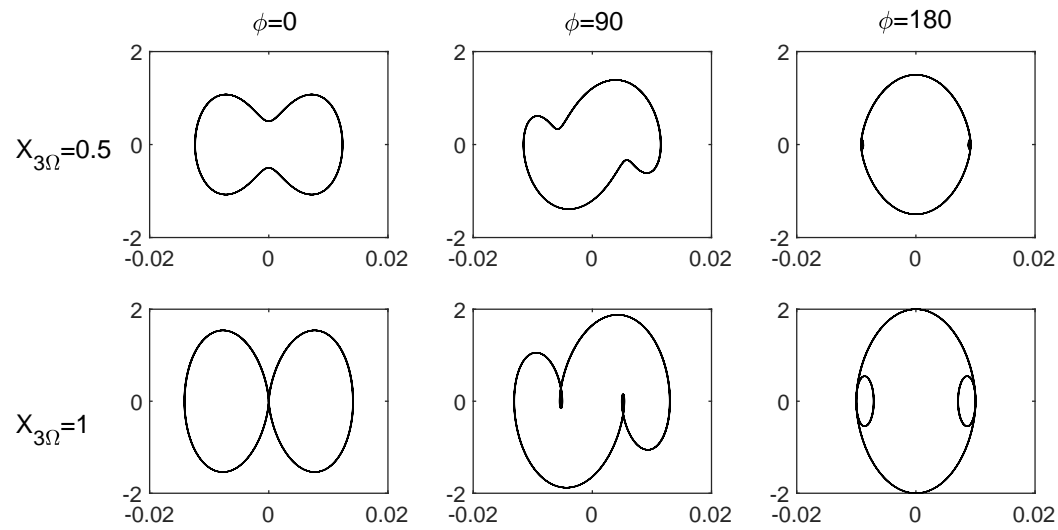

Figure 6.6: Reference phase portraits for variations on amplitude and phase at $3 X$. The horizontal axis corresponds to velocity, and the vertical axis to acceleration.

The effect of phase on the shape of the portraits id highly significant. It is seen that the presence of $X_{3 \Omega}$ without phase change leads to a constriction of the ellipse. The $\pi / 2$ phase difference shows as a skewed distortion and the inner loop for $X_{3 \Omega}=1$ while the $\pi$ phase difference shows the ellipse with inner loops. These differences are taken as the basis for comparing different failure states.

\subsubsection{Load distribution analysis}

The last method used analysed the internal load distribution of the beam. This is achieved by quantifying the participation of each of the three vibration modes (i.e. $1 R B, 1 F E$ and $2 F E$ ) at the different harmonics of the vibration response (i.e. $1 \Omega$, $2 \Omega, 3 \Omega$, etc.).

The natural frequencies for the rigid body and the first and second elastic modes are shown in Table 6.2. The rigid body frequency was characterised through the impact test only as the frequency $(i \mathrm{i} 10 \mathrm{~Hz})$ was bellow the shaker range. The first flapping frequency $1 \mathrm{FE}$ was characterised by the two procedures. There is a slight variation between the results, but these could be attributed to the influence of the shaker. The second flapping mode was characterised by the sweep test only.

The values presented in table 6.2 show a close similarity between the different cases for the rigid body and first flapping frequency. A slightly higher differentiation occurred at the second flapping mode, specially for $T S 1$. This difference did not show significant for the later analysis. The mode shapes estimated through the sweep test are shown in Figure 6.7. 
Table 6.2: Natural frequencies for the rigid body, first and second elastic flapping modes of the test beam.

\begin{tabular}{lllll}
\hline & Impact & \multicolumn{3}{l}{ Sweep } \\
\hline & RB [Hz] & 1EF [Hz] & 1EF [Hz] & 2EF [Hz] \\
Ref & 8,7 & 42,3 & 44 & 100 \\
TS1 & 8,4 & 42,9 & 44 & 90 \\
TS2 & 7,7 & 44 & 46 & 115 \\
Ref-AM & 6,9 & 44,7 & 45 & 98 \\
TS1-AM & 7,5 & 42,1 & 44 & 113 \\
TS2-AM & 7,4 & 43,7 & 45 & 115 \\
\hline
\end{tabular}

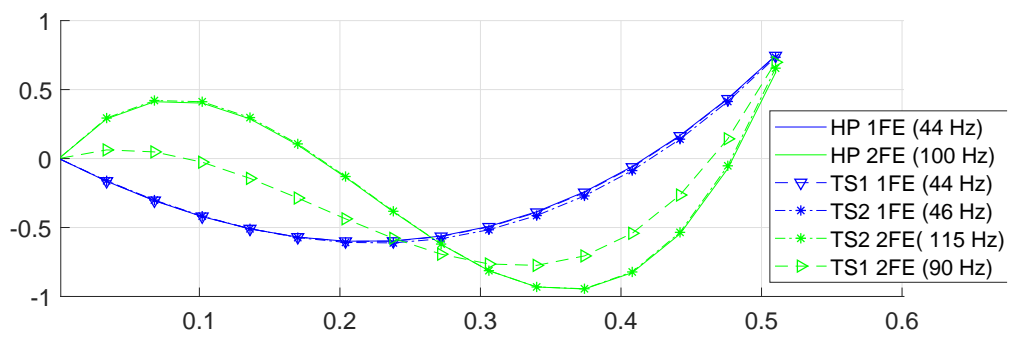

(a) No added mass

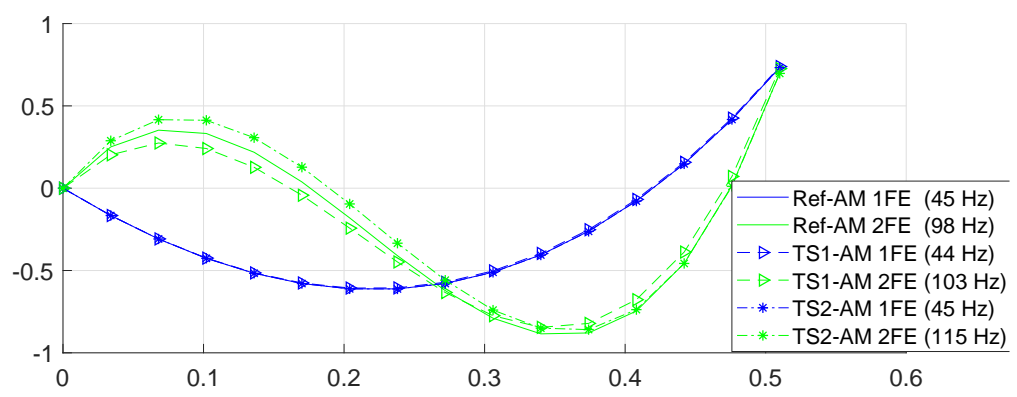

(b) With added mass

Figure 6.7: First and second mode shapes for test beam under different torsional stiffness. 
Figure 6.8 presents the harmonic analysis for the reference case. The time waveform for the measurement points and the spectrum for the tip response are shown in the top frames. The lower left frame shows the ODS for the most significant peaks identified. The lower right frame shows the absolute value of the participation factors for each flapping mode.
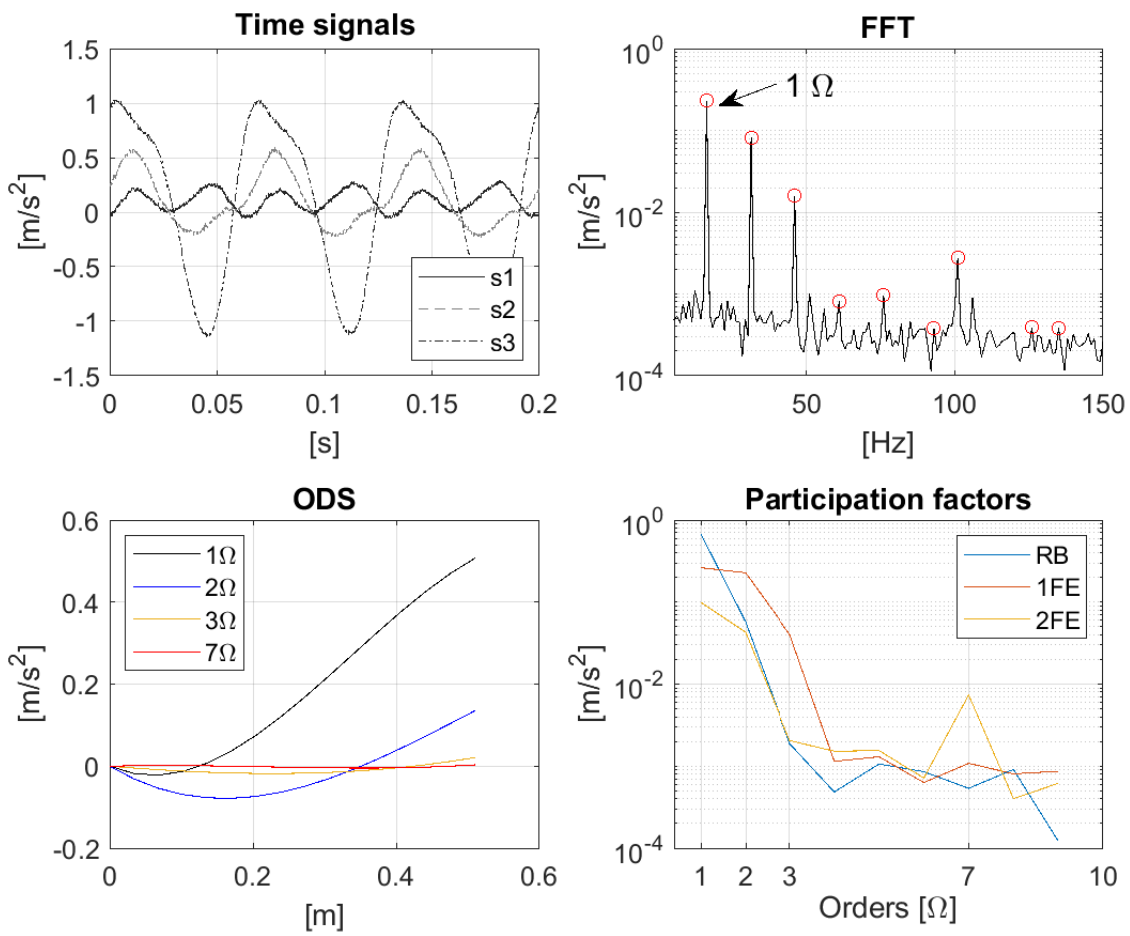

Figure 6.8: Harmonic analysis for reference case.

The participation factors (lower right frame) are analysed with special attention at orders $1 \Omega, 2 \Omega, 3 \Omega$ and $7 \Omega$. For the reference case, it is seen that the rigid body $R B$ appears stronger at $1 \Omega$, and the first flapping mode $1 F E$ is more active for $2 \Omega$ and $3 \Omega$, followed by a fall in vibration activity. The second flapping mode $2 F E$ shows a moderate presence at $7 \Omega$ coinciding with its natural frequency. 


\subsection{Results}

This section presents the evaluation of the beam response for the different test configurations. The analysis for the individual failure modes is presented first, followed by the failure state dependency.

\subsubsection{Characterisation of individual modes}

The beam response at the excitation frequency is analysed as described in Section 6.3.1. The forced excitations for each of the individual test variables are analysed for the change of $X_{1 \Omega}$. Figure 6.9 shows a comparison of the values of $X_{1 \Omega}$ with respect to the reference case. It is seen that all the individual cases display lower values than the reference. This implies that the reference case holds the highest linearity among the test cases. Note that the largest variation is found for high lagging $(L 2)$.

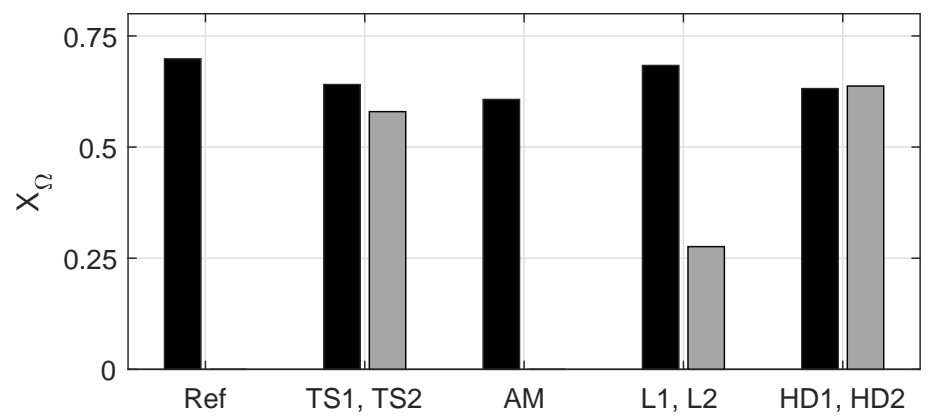

Figure 6.9: Comparison of normalised $X_{1 \Omega}$ for individual test cases.

The structural variations, corresponding to variation of torsional spring and added mass are analysed further. Figure 6.10 presents a comparison of the time waveform, spectral distribution, TKEO spectrum and the phase portraits. The TKEO spectra (introduced in Chapter 5) indicate the most important modulation in the signal. For all the cases presented this is shown at $15 \mathrm{~Hz}$. Similarly the phase portraits show that all cases display an elliptical shape. This means that the structural variations due the torsional spring and the added mass do not cause a significant effect with respect to the reference case.

The combinations of medium and soft torsional springs with added mass are shown in Figure 6.11. It is seen that the combination $T S 1-A M$ displays a similar behaviour to the independent cases. Instead the combination TS2-AM shows a more unstable behaviour, reflecting a different failure state. 
Ref
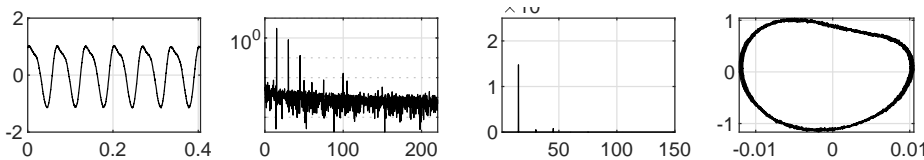

TS1
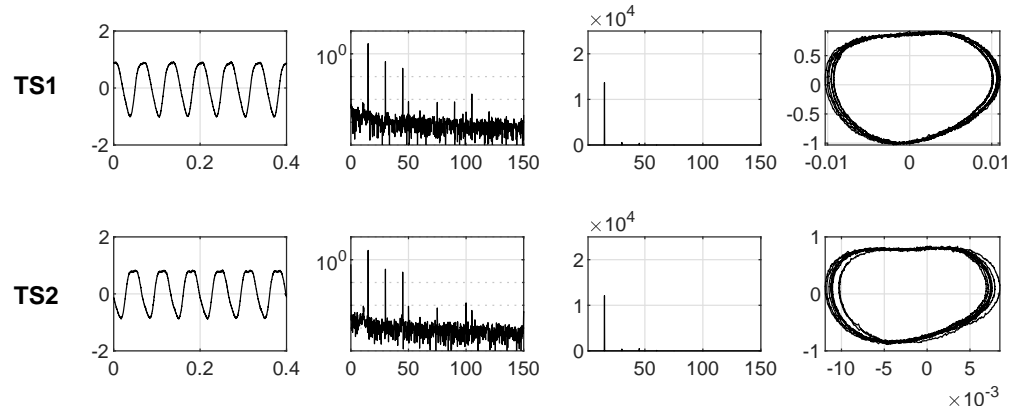

AM
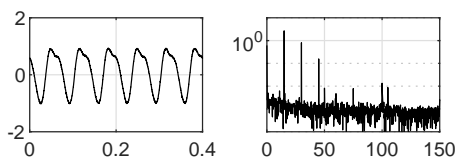
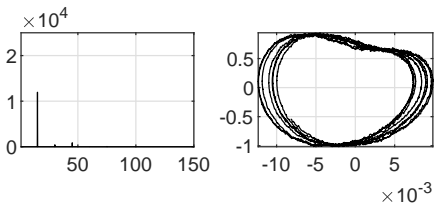

Figure 6.10: Tip response analysis for reference and structural variations cases.

Figure 6.12 shows the tip response analysis for the excitation input changes. These test conditions display a more differentiated effect in comparison with the structural variations. The response to low lagging $(L 1)$ displays the fewest deviations with respect to the reference and structural variations. Conversely, the high lagging ( $L 2)$ shows an increased participation of higher harmonics on the signal. Specifically the TKEO reveals high modulation at $105 \mathrm{~Hz}$ (i.e. $X_{7 \Omega}$ ) corresponding with the second flapping mode. This implies a coupling between the second flapping mode and the lagging excitation, probably due to a torsional component in the flapping mode.

The response to the harmonic distortion shows, as expected, an increment in the third order $X_{3 \Omega}$ (i.e. $45 \mathrm{~Hz}$ ). The amplitude of $X_{3 \Omega}$ shows a similar magnitude to $X_{1 \Omega}$, despite the fact that the input was only $10 \%$ of the reference excitation. The addition of $X_{5 \Omega}$ for $H D 2$ does not show a significant effect on the response.

The ODS and participation factors for the lagging and harmonic excitations are presented in Figure 6.13. For the lagging excitation $(L 1)$ it is seen that the first order $(1 \Omega)$ a higher participation in the ODS than the other orders. The harmonic distortion (HD1) shows a balanced response as seen for the first three orders. 

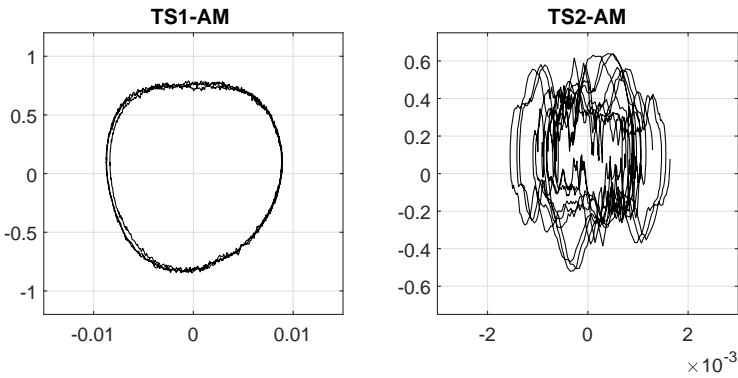

Figure 6.11: Combined effects of added mass and a) medium torsion spring $b$ ) softer torsional spring.
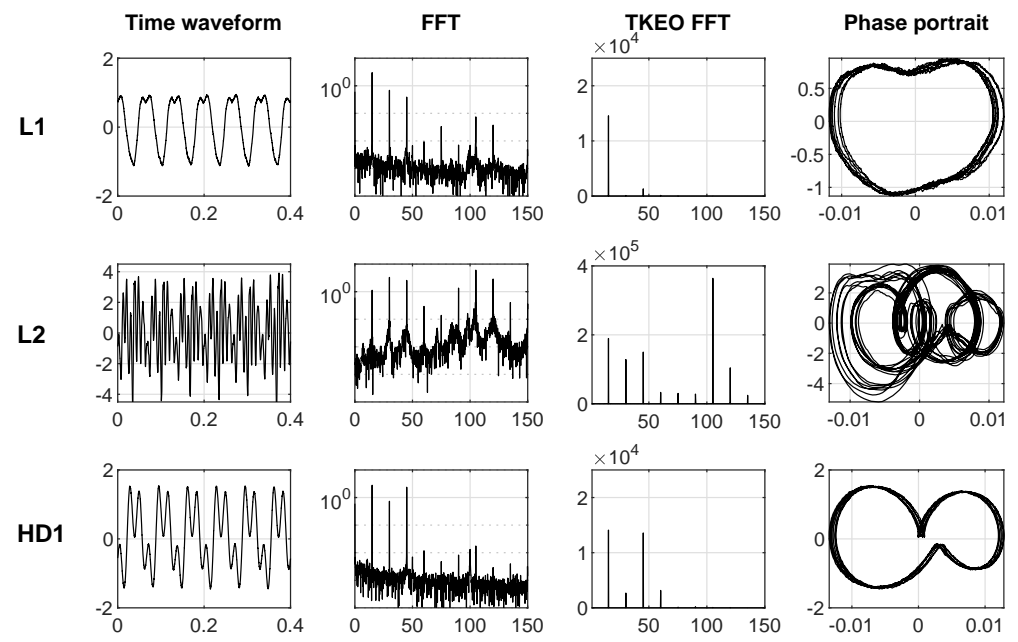

HD2
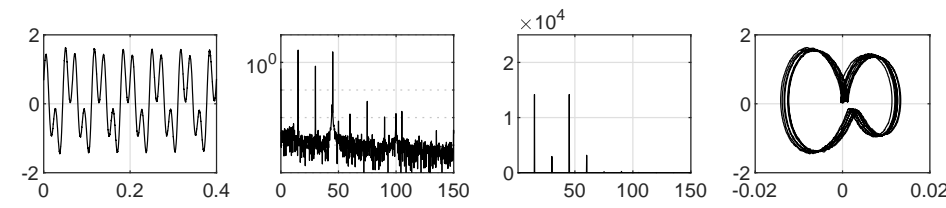

Figure 6.12: Tip response analysis for excitation input variations.

The participation factors show the effect of the flapping modes on the response. For the lagging case it is seen that the second mode has a local maximum at $7 \Omega$, confirming the coupling identified at $L 2$ in Figure 6.12. For the harmonic distortion 
ODS

L1
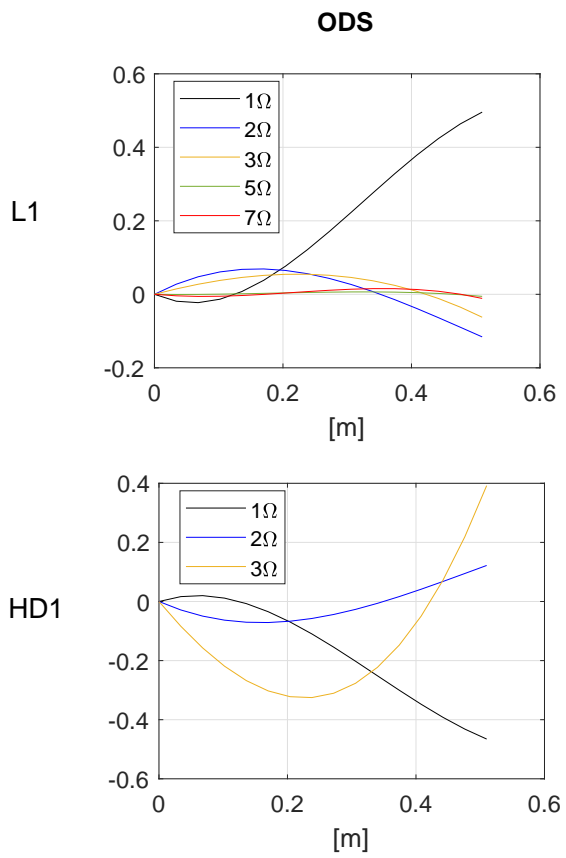
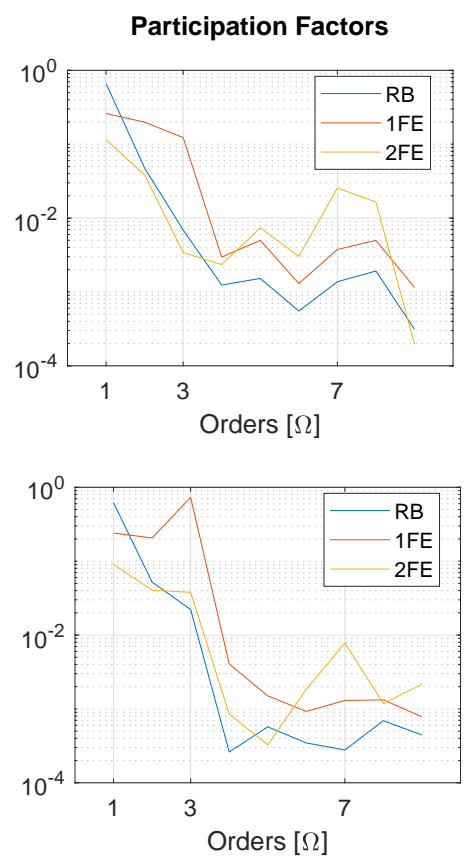

Figure 6.13: Comparison of the load distribution for lagging and harmonic distortion. left) ODS, right) participation factors.

it is confirmed that the response at $3 \Omega$ obeys mainly the first elastic mode. From the comparison of the input excitations responses it is concluded that the lagging and harmonic distortion display low dependency, as these affect distinct structural modes.

Based on the triangle in Figure 6.4, Figure 6.14 shows the phase portrait for the different individual test cases. In this Figure it is seen that several responses (i.e. $T S 1, T S 2, A M$ and $L 1$ ) display similar characteristics to the reference state (Ref). For the case of lagging, it is seen that the amplitude of the excitation ( $L 1$ and $L 2)$ leads to two different failure states. Finally, the lagging and harmonic distortion are shown to be independent as these affect different mode shapes. 


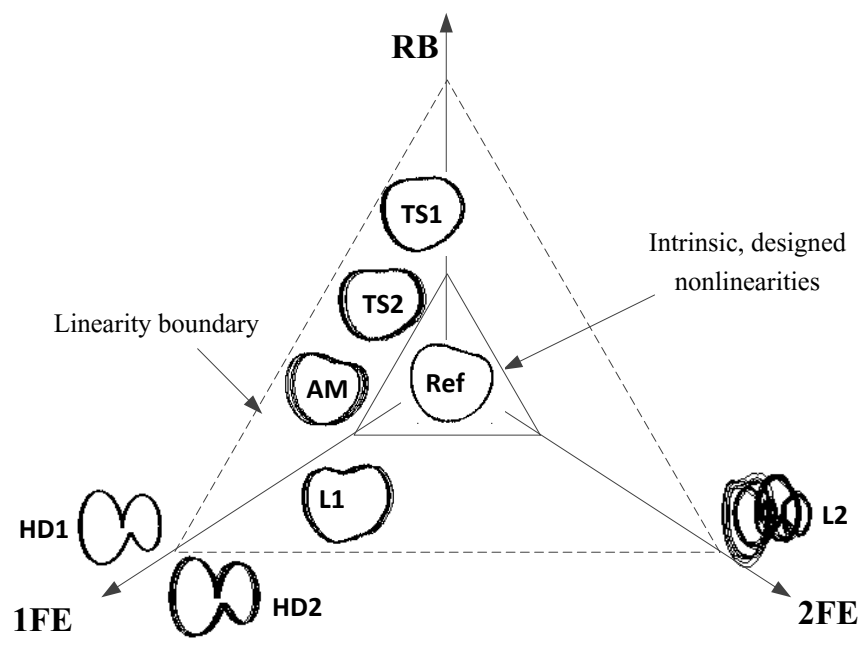

Figure 6.14: Representation of the different failure states due to the individual effect of the test cases.

\subsubsection{Mode-state dependency analysis}

The dependency of the harmonic distortion with respect to various failure state combinations is analysed according to the procedure shown in Figure 6.5. Figure 6.15 presents the variation of $X_{1 \Omega}$ for the two sets of $\Theta_{i}$. The black bars correspond to the base failure state and the grey bars to the emerging failure state under the influence of harmonic distortion. Figure 6.15a shows the structural variations. It is seen that the reference case displays the largest $X_{1 \Omega}$ response for all cases. This confirms that the reference has the highest linearity. Furthermore, the addition of harmonic distortion causes a slight reduction in the value of $X_{1 \Omega}$.

The addition of lagging leads to a comparatively higher variability of $X_{1 \Omega}$ as shown in Figure 6.15b. This shows an increased nonlinearity in the system as the excitation of the lagging direction influences the flapping direction effectively. This is most critical for the combination of added mass and lagging, where the $X_{1 \Omega}$ value decreases substantially. Instead, the combinations softer torsional and lagging $(T S 1+L L)$ shows a slightly higher value than the reference.

Moreover, the addition of the harmonic distortion makes the final state less predictable. Thus, while for $\operatorname{Ref}+L 1$ and $T S 1-A M+L 1$ the value of $X_{1 \Omega}$ decreases, 


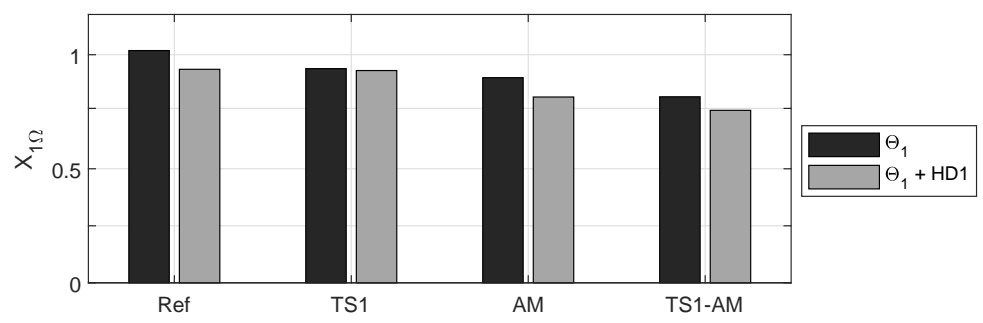

(a) Variations in the structural configuration

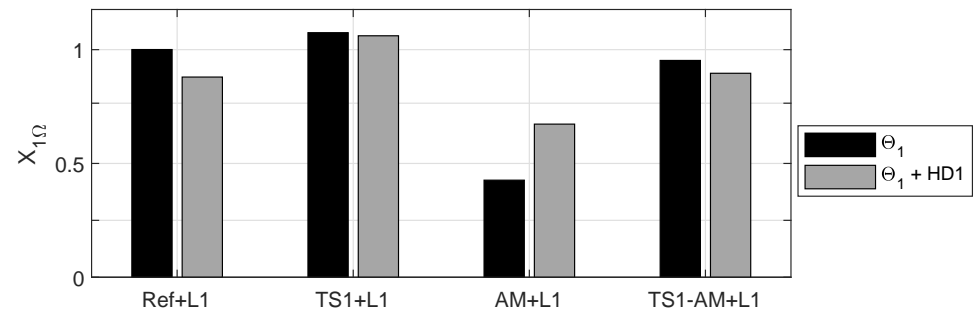

(b) Addition of lagging

Figure 6.15: Comparison of normalised tip response at $X_{1 \Omega}$ for different base failure states (black) and with the addition of harmonic distortion (grey).

it increases for $A M+L 1$ and remains unchanged for $T S 1+L 1$. This illustrates the difficulty of predicting system behaviour once the failure increases system nonlinearity.

An overview of the phase portraits for the different transitions is presented in Figure 6.16a. It is seen that the base states show little variability as most retain the ellipse shape. The only exception is $T S 1+L L$, which shows a significant reduction in the ellipse size consistent with the $X_{3 \Omega}$ reduction, and a slight constriction and rough delineation. 

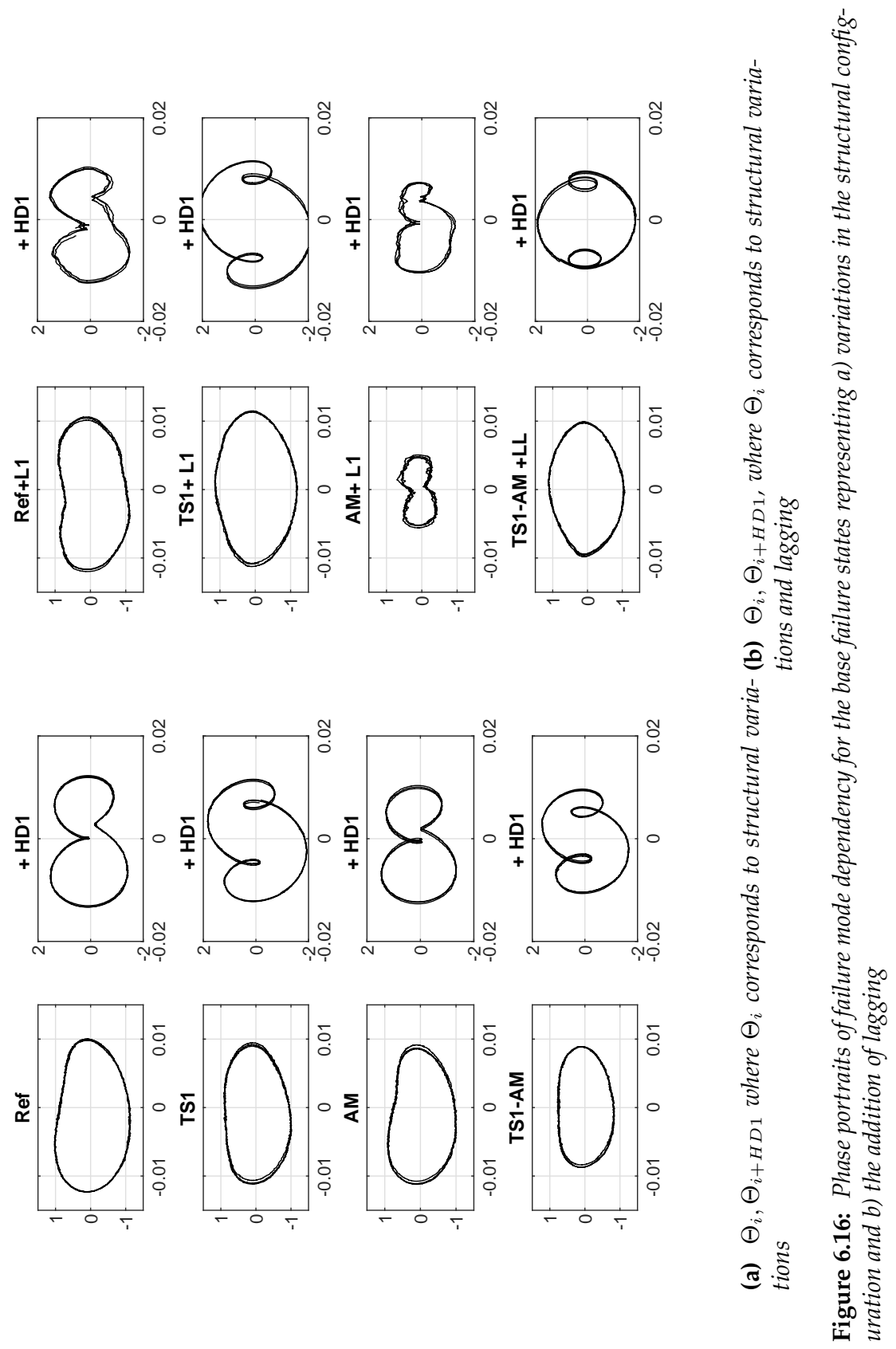
The addition of harmonic distortion as incipient failure mode shows very distinct variations on the phase portraits including constrictions and inner loops. The additional loops take place when the base state includes the medium torsional stiffness (TS1). Conversely, the addition of lagging causes more profound differentiation. The highest variation is $T S 1-L 1+H D 1$, which has two well-defined inner loops similar to the condition $\phi=180$ in Figure 6.6.

Figure 6.17 presents a mapping of the phase portraits for the magnitude and phase $(\phi)$ of the third order $X_{3 \Omega}$ for different combinations of external inputs, based on the equation 6.2. The variations are considered for the case where no structural variations are enforced (Ref) and for the combination of medium torsional spring and added mass $T S 1-A M$. For the reference case (Figure 6.17a) the diagram shows that the addition of lagging and harmonic distortion independently does not change the phase considerably. Instead, the combined effect leads to a delay of $\pi / 2$.

For the combination of medium torsional spring and added mass (Figure 6.17b), the addition of harmonic distortion shows internal loops and the phase delay increases. Furthermore, the most significant increase in both amplitude and phase occurs when lagging and added distortion are excited simultaneously. This state shows a phase difference of $\pi$, confirming the resonance of the first flapping mode.

The participation factors for different combinations of structural variations, lagging and flapping are presented in Figure 6.18. For the combination $R e f+L 1+H D 1$ it is seen that the lagging and harmonic distortion affect the first and second mode respectively. This confirms that the failure modes appear to act independently when acting over the reference condition, as initially shown in Figure 6.13. This independence is still observed at the combination $A M+L 1+H D 1$, yet the effect of added mass reduces the magnitude of the participation factors for all the modes.

The reduction in the torsional stiffness (TS1) shows a higher participation of the first flapping mode $(1 F E)$ at the $T S 1+L 1+H D 1$ and $T S 1-A M+L 1+H D 1$. Additionally, a lower participation of $2 F E$ is seen. The main difference between these two cases is that for the latter all the modes display a local maximum at $X_{3 \Omega}$, explaining the resonance condition.

The lower participation of the second flapping mode for the combination with added mass and medium spring is investigated next. Figure 6.19 shows the combination of medium stiffness and added mass for the two lagging excitation levels $(L 1$ and $L 2)$. It is seen that the lower excitation level for $T S 1+L 1$ and $T S 1-A M+L 1$ does not reveal any significant change from the ellipse shape. However, the higher excitation level leads to an increment in the second and third harmonic, as shown for $T S 1+L 2$ and $T S 1-A M+L 2$, which differs from the high excitation at $X_{7 \Omega}$ for the case $+L 2$ shown in Figure 6.12 . 


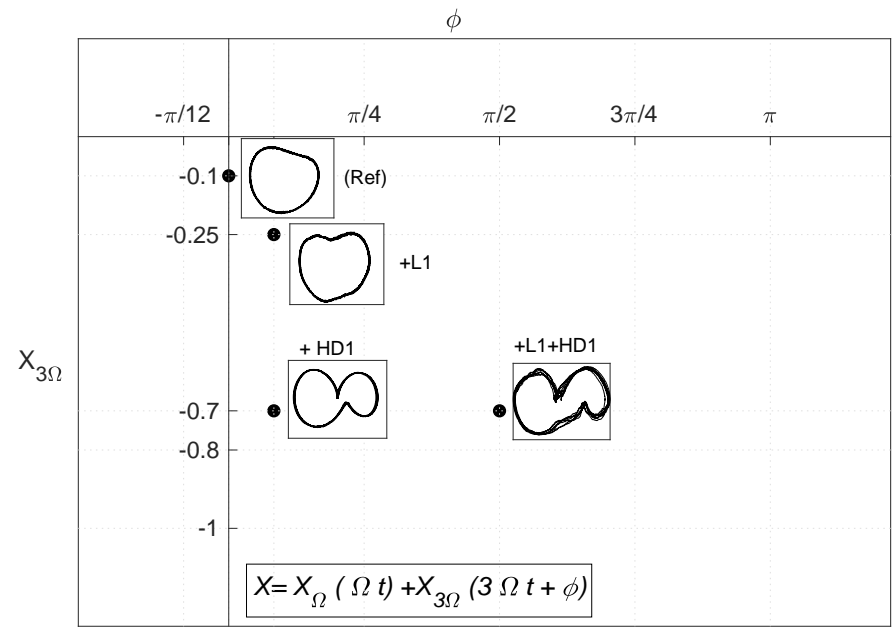

(a) $\Theta_{i+H D 1}$ for different combinations based on the hardest torsional spring.

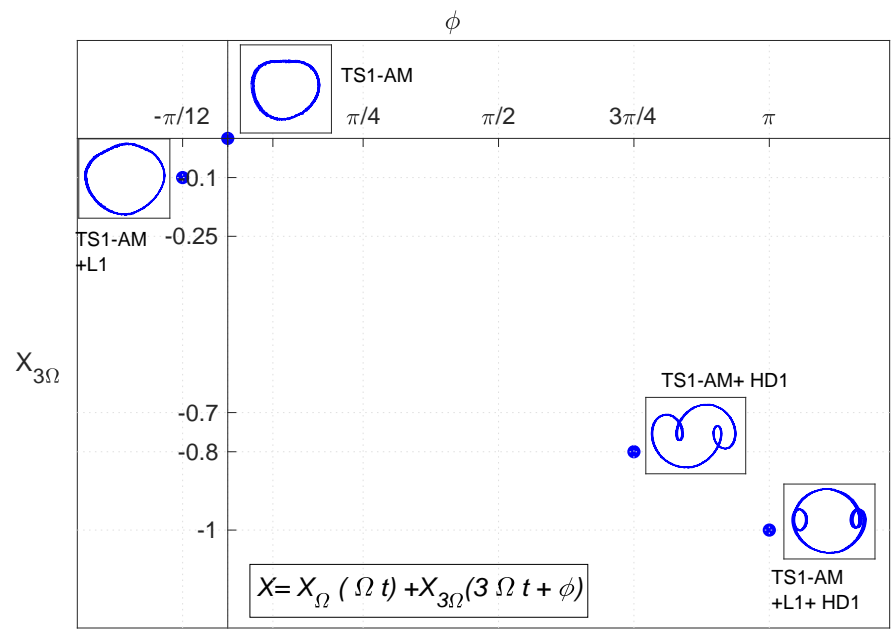

(b) $\Theta_{i+H D 1}$ for different combinations based on the medium torsional spring and added mass

Figure 6.17: Mapping of magnitude and phase for $X_{3 \Omega}$. 

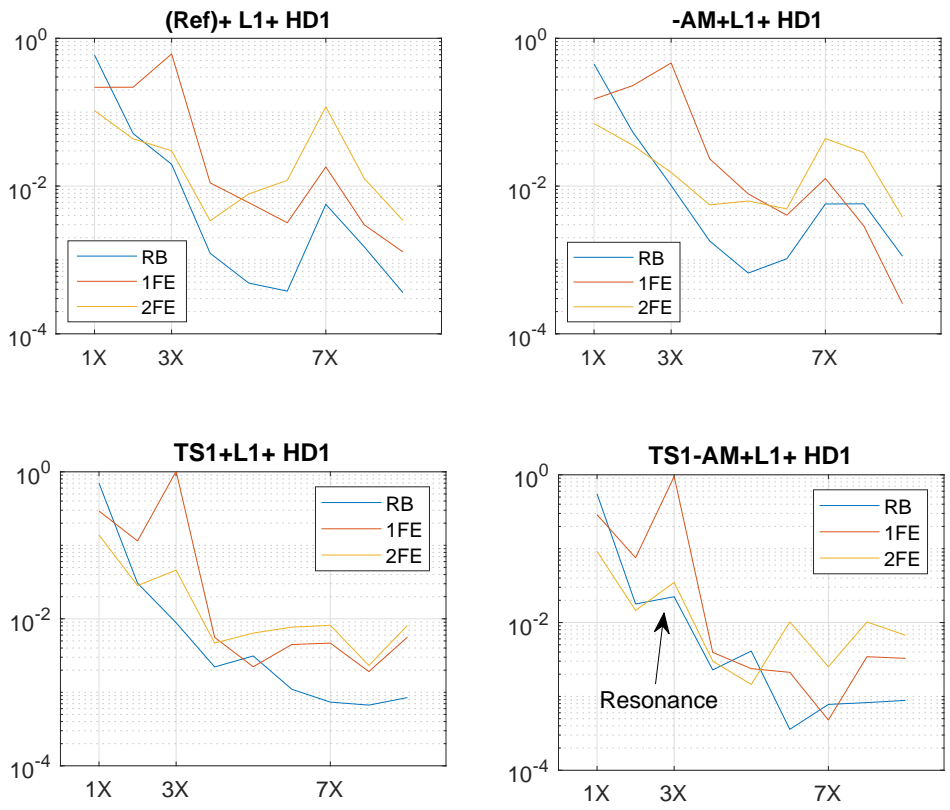

Figure 6.18: Comparison of participation factors for different combinations including lagging and harmonic distortion

This is explained as follows. It was expected that the lower boundary stiffness would increase the participation of the elastic modes in comparison to the rigid body mode, and that added mass would dampen the higher harmonics. Yet the analysis of lagging shows that the combination of medium torsional spring leads to the excitation of the first mode shape. Note that this realisation was only possible with the higher lagging excitation. Thus the lagging and harmonic distortion contribute simultaneously to the first flapping mode, leading to a resonance state as shown in Figure 6.20. 

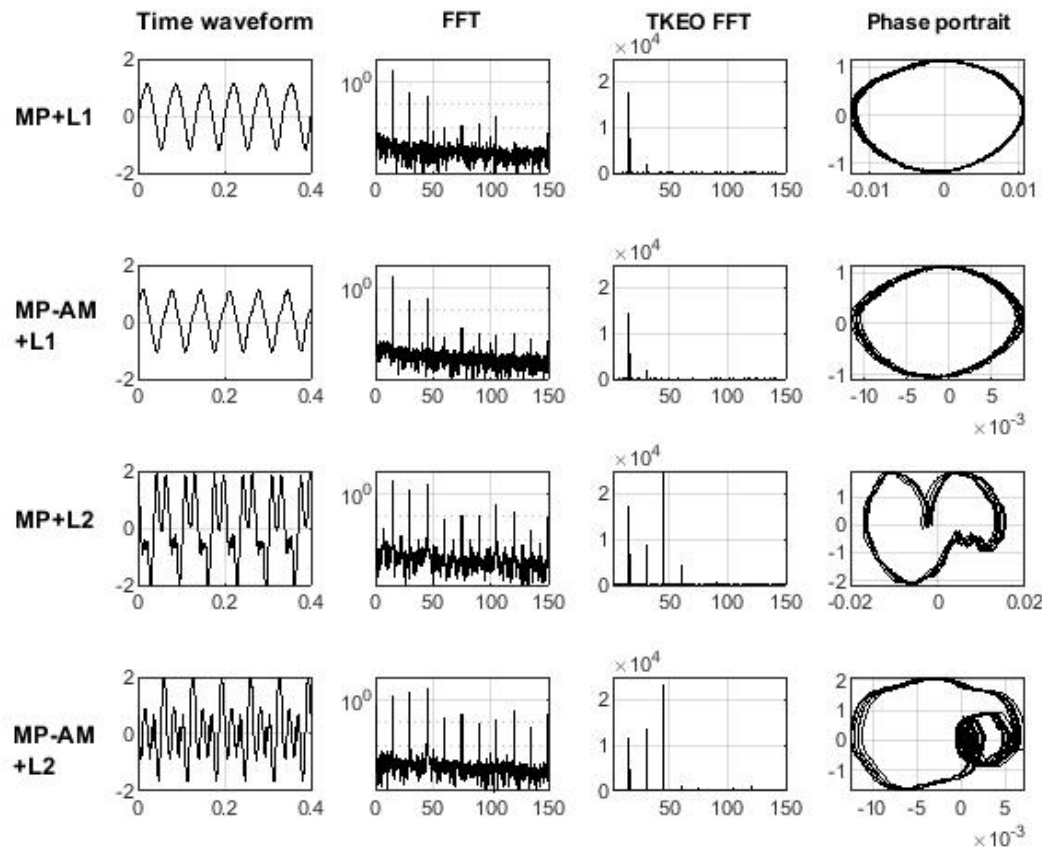

Figure 6.19: Tip response for medium torsional spring, added mass and lagging.

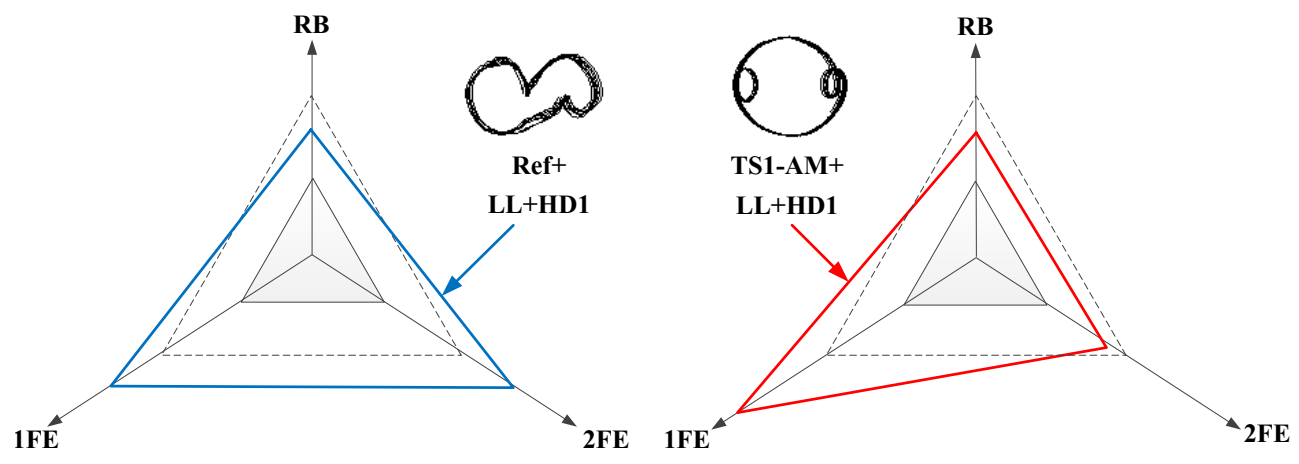

Figure 6.20: Comparison of the effect of lagging and harmonic distortion when acting over the reference case and on over the medium torsional spring. 


\subsection{Discussion}

The presented experimental study illustrates the application of the functional approach to vibration analysis applied on rotor blade systems. The current approach to assessment of beam-like structures focuses on the characterisation of local damage based on the spatial aspects of the vibration response. The study presented considered the spatial and temporal aspects of the beam response, providing a more realistic representation of actual rotor blade systems. This combined approach enabled the detection and identification of the nonlinear behaviour, as well as the characterisation of the load distribution changes responsible of the observed nonlinearities.

The combined effect of multiple failure modes in rotor blade systems was analysed. It was seen that each failure mode led to a different effect on the blade response, yet the intensity necessary for a new failure state varied. Furthermore, the interactions between failure modes were investigated. It was found that, although structural changes did not lead to significant variations in the input response, their contribution was decisive for triggering new failure states when combined with changes in the input excitations. For instance, lagging and harmonic distortion showed independent behaviour by triggering different nonlinear responses on the system, even when excited simultaneously, i.e. it was possible to distinguish the individual contributions from both failure modes. Yet, when acting in combination with the medium torsional spring, the total response was significantly different, reaching a resonance state.

It must be noted that the proposed methods were possible by directly sensing at the beam, yet for actual rotating blades this type of instrumentation is uncommon. Nevertheless, even with a restricted number of sensors, it was possible to derive significant information about the beam response. In fact the tip sensor alone provided great detail of the nonlinear phenomena taking place. This shows the ample possibilities for more comprehensive assessment of rotor blade systems using on-blade sensors.

\subsection{Conclusion}

The experimental study ot the rotor blade demonstrator shows how complexity increases in systems undergoing failure. It was shown how with each nonlinear transformation the system acquired a new set of characteristics different from those expected for the designed behaviour. This means that the progressive changes in the system behaviour reinforced the difficulty of distinguishing each participating failure mode's contribution. 
Nevertheless, the presented experimental study provided an example of how to address complex failure. Individual failure modes were investigated for distinguishing the affected functional features and for quantifying the excitation level needed to trigger new failure states. This investigation proved supportive for the interpretation of emerging failure states when combining multiple failure modes. In sum, the experimental set-up provided a qualitative model of the nonlinear changes in the dynamic behaviour attributed to failure.

Qualitative models, both physical - as the demonstrator used in this chapter and numerical, are necessary for the assessment of systems undergoing complex failure. These provide a sound basis for the identification of the constituent failure modes and for the characterisation of the failure states that are most likely to to emerge. In conclusion, a combined approach of qualitative models with observationbased quantitative models, as presented in the previous chapter, is recommended for more accurate predictions of the failure evolution. 


\section{Part III}

\section{Design implications and Closing}





\section{Chapter 7}

\section{Vibration monitoring systems}

The final part of this thesis addresses the implications of the proposed functional approach for the design of vibration monitoring systems. Vibration monitoring systems are defined as the organisation of instruments and algorithms required for transforming vibration signals into useful information about the system condition. The transformation from signals into information requires intermediate steps from signal acquisition and signal processing, to data reduction and evaluation.

The deployment of a vibration monitoring system comprises two main aspects: the monitoring implementation defined by the physical instrumentation (e.g., sensors, cabling) that transforms the physical signal into digital data, and the monitoring strategy defined by the algorithms responsible to transform the data into useful insights about the specific maintenance requirements.

The realisation of the functional approach to vibration analysis requires a combined agreement between the monitoring strategy and implementation. However, as most industrial vibration strategies focus on the early damage detection the operational vibrations are treated through simpler features (e.g. rms values). The physical monitoring implementation is also a limiting factor for realising a functionalityfocused vibration monitoring. As seen in the roller bearing and rotor-blade cases, the ability to interpret load distribution changes is conditioned to the accurate register of the temporal and spatial aspects, especially in variable operational environment.

It is argued that the main limitation for extending the focus of the monitoring systems beyond damage is the disconnection between the monitored and monitoring systems. Consequently, both the strategy and implementation are conducted without considering the monitored object's functionality. 
This chapter presents a design framework for function based vibration monitoring systems. Based on the characteristics of failure behaviour discussed in Part I, the design problem is defined as the development of a system whose main function is to monitor the divergence from the designed state of a rotating mechanical system. The framework adopts the Function-Behaviour-Structure (FBS) formulation to identify the interdependencies between monitored and monitoring systems, as shown in Figure 7.1. The main goal of framework is to support stakeholders on the requirement definition for the monitored strategy and implementation.

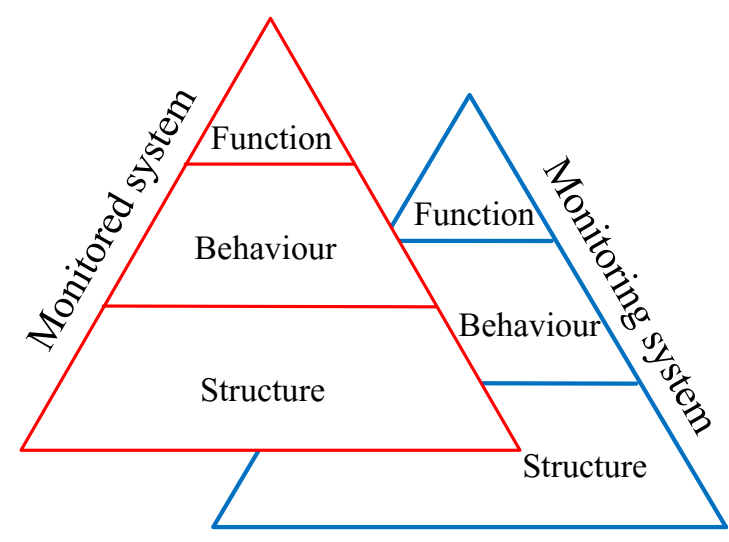

Figure 7.1: FBS for the monitored and monitoring systems.

This Chapter is organised as follows. Section 7.1 presents the Function-BehaviourStructure for the monitored and the monitoring systems. The definition of system requirements is presented in Section 7.2. Conclusions are presented in Section7.3.

\subsection{Design framework}

The Function-Behaviour-Structure formulation [37] is guided by three questions about the system to be designed: function: what the purpose of the object is; behaviour: what the object does; and structure: what the object consists of. For the design of monitoring systems, these questions must be re-formulated to include the monitored system.with respect to This means that the requirements are defined not only towards the configuration of the monitoring system, but also to support the interrogation of the observed system. 


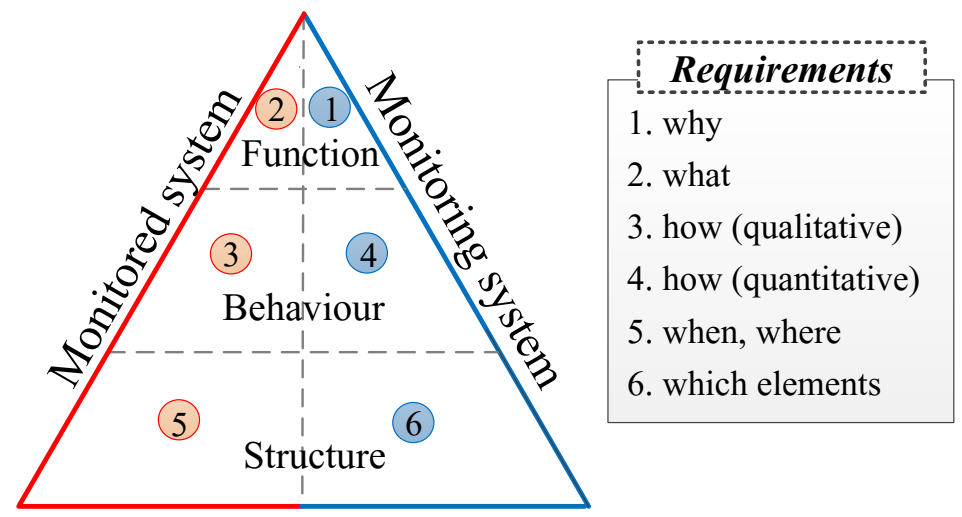

Figure 7.2: FBS-based requirements for vibration monitoring systems.

Figure 7.2 presents a visualisation of the integration of the function, behaviour and structure levels from the monitored and monitoring system. An extended set of questions that address the correspondence between the systems is proposed:

- why is the monitoring of this system necessary?

- what is the functionality of the object to be monitored?

- how (qualitatively) is the machine changing its behaviour due to failure?

- how (quantitatively) is the failure behaviour reflected in the vibration behaviour?

- when and where are the sensing and local processing tasks required?

- which monitoring configuration (i.e. instruments, network structure) is the most suitable for the implementation?

The following sections address these questions as guidance for the integration of the monitored and monitoring systems. 


\subsubsection{Monitored system}

The goal of the FBS for the monitored system is to outline the vibration response for the most likely failure states of the investigated system [114]. This means providing a qualitative estimation of the nonlinear phenomena expected as a consequence of failure, and approximating quantitatively the frequencies and magnitude of the derived vibration response. Figure 7.3 shows the application of the FBS concept to the monitored system. This representation illustrates the concept of divergence between the expected and observed behaviour, as introduced earlier in Figure 3.1. The vertical line represents vibration behaviour at the designed state and the red line at a given failure state. The upper layer represents the overall system functionality and the lower layer the material failure mechanisms.

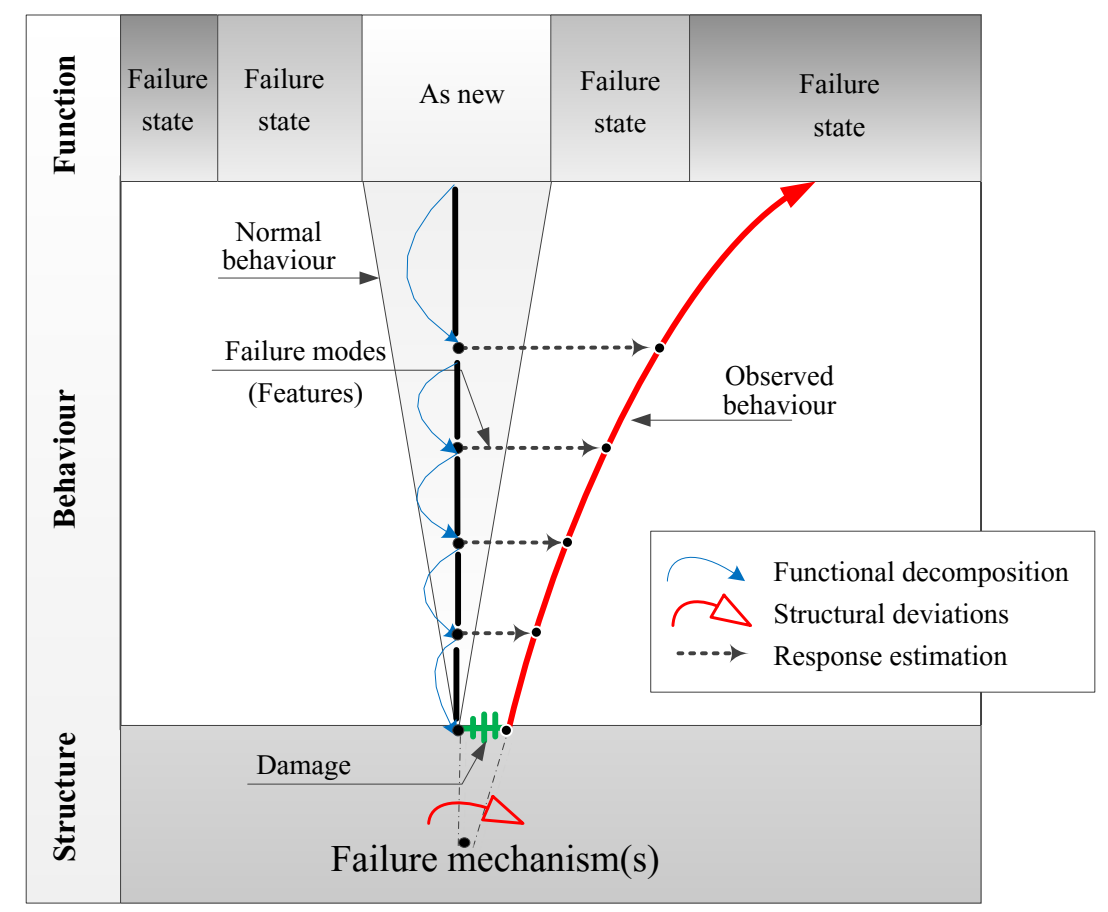

Figure 7.3: FBS for the monitored system 
Figure 7.3 shows three steps that are necessary for the estimation of failure behaviour: the functional decomposition from system to component level, the estimation of how the failure mechanisms affect the component functionality, and the prediction of features representing the identified failing functions. These are explained in detail below.

\section{Functional decomposition}

This step identifies how the main goals defined at the system level are realised through subsystems, assemblies and finally components [115-117]. The component functions are classified according to the functional hierarchy presented in Section2.3.1. Functional dependencies must be identified at this level, enabling the prediction of the most likely sequences of failure proliferation.

\section{Structural deviations}

The second step is the characterisation of the effects of the material degradation for individual components. The analysis must also include the identification of the most likely failure mechanisms and their likely interdependencies, as introduced in Section2.1.2. Next, the dependency between performance and reliability parameters for the linear and nonlinear ranges, as discussed in Section2.2.2.

\section{Response estimation}

The last step synthesises the main insights of the previous steps to anticipate how the possible failure modes reflect on the vibration behaviour. This means to estimate the change in the operational forces and structural modes, and the emergence of damage-induced modes and forces. Finally, the combined effects of multiple failure modes for new emerging failure modes must be assessed.

\subsubsection{Monitoring system}

As mentioned before, the monitoring system can be defined as the set of instruments (structure) and algorithms (behaviour) that provide information (function) as input for maintainer's decision-making regarding the monitored object condition. Each of these FBS layers displays a reciprocal relation with the monitored system: the ultimate function of the monitoring system is to support maintenance decisions that ensure the functionality of the monitored system, the algorithms must be adapted to 
the vibration behaviour, and the instrumentation must be deployed considering the physical configuration of the monitored system.

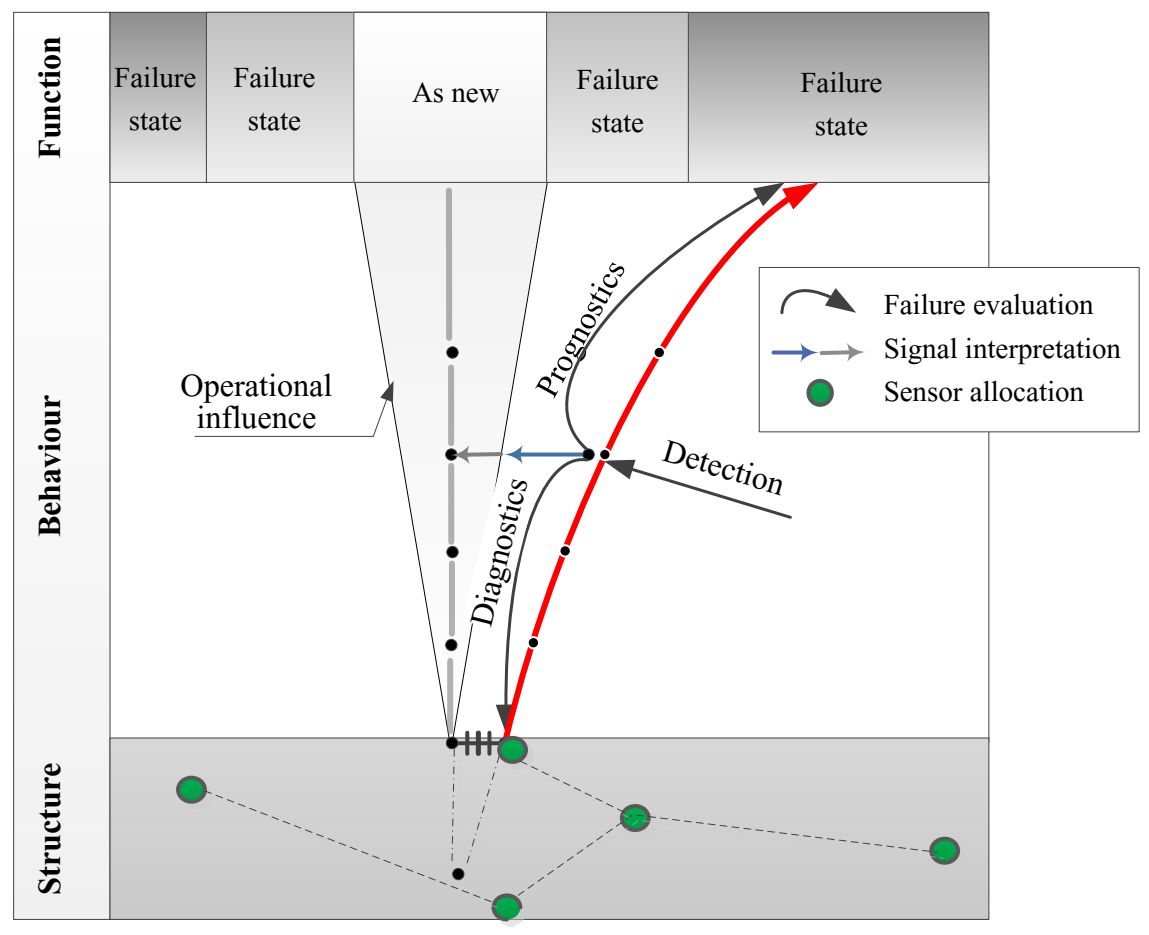

Figure 7.4: FBS for the monitoring system

The FBS for the monitoring system is represented in Figure 7.4. It is shown that the monitoring activity results from the integration of the failure behaviour evaluation in relation to detection, diagnostics and prognostics, the interpretation of the vibration response, and the distribution of the sensors at the system structure. These tasks are discussed in detail next. Special attention will be paid to the case of distributed sensing as a path for improved registration of load distribution changes. 


\section{(Complex) failure evaluation}

The motivation for establishing a vibration monitoring system resides in the capacity to plan effective maintenance interventions that guarantee system functionality. Typically these motivations are categorised as detection of abnormal failure, the diagnostics of the responsible failure modes and failure mechanisms, and the prognostics of remaining useful life. However, for complex failures a more extended descriptions for these goals are required, in line with the questions presented in Figure 4.1.

A general formulation of monitoring needs is introduced next. The detection stage focuses on the existence of damage, which requires distinguishing damagerelated effects from environmental and operational influences on the vibration signal. The aim of diagnostics lies on verifying the origins of the deterioration path that has led to the detected abnormality, specifically whether the failure is likely related to a simple failure, a system-level complicated failure, or part of a complex failure. Finally prognostics aim to estimate the risks associated with the proliferation of failure from the current failure state. These expectations must be formulated as requirements for the intensity and scope of the monitoring activity.

\section{Signal interpretation}

This level constitutes the core of a vibration monitoring system, as a motivated connection between failure behaviour and vibration is established here. This implies the validation of the expected predicted features from the FBS of the monitored system, and the qualitative assessment of their magnitude. Simultaneously, the three goals of detection, diagnostics and prognostics are paired with the features of the vibration response. This means that the detection of abnormalities is achieved by relating the measured features to the expected behaviour of the system, including the variations due to the operational environment. The diagnostics is performed by reasoning back from the observed feature to the potential failure mechanisms on the component level. And finally prognostics is achieved by extrapolating the observed features for the transition to a new failed state.

\section{Sensor allocation}

Finally, the structure level refers to the deployment of the instrumentation with respect to the system configuration (physical structure) and its use. This means when and where to measure. As discussed before, traditional damage-oriented monitoring instrumentation for rotating machines is insufficient to disclose the load distribution 
changes associated with complex failure. The centralised nature of the current implementations limits the fast response to the variation of the operational environment and the coordination between different sensor locations.

Distributed sensor networks are devised as a solution for the monitoring of complex failures $[118,119]$. The difference between centralised and distributed sensor networks (DSN) is illustrated in Figure 7.5. In the centralised systems the sensor elements report directly to a central processing unit. In a general DSN structure there are additional processing elements which are interconnected through a communication network. Data is transferred from sensors to their associated processing elements, where the data integration takes place. The processing elements can also coordinate with each other to achieve a better estimation of the environment and report to the central processing.

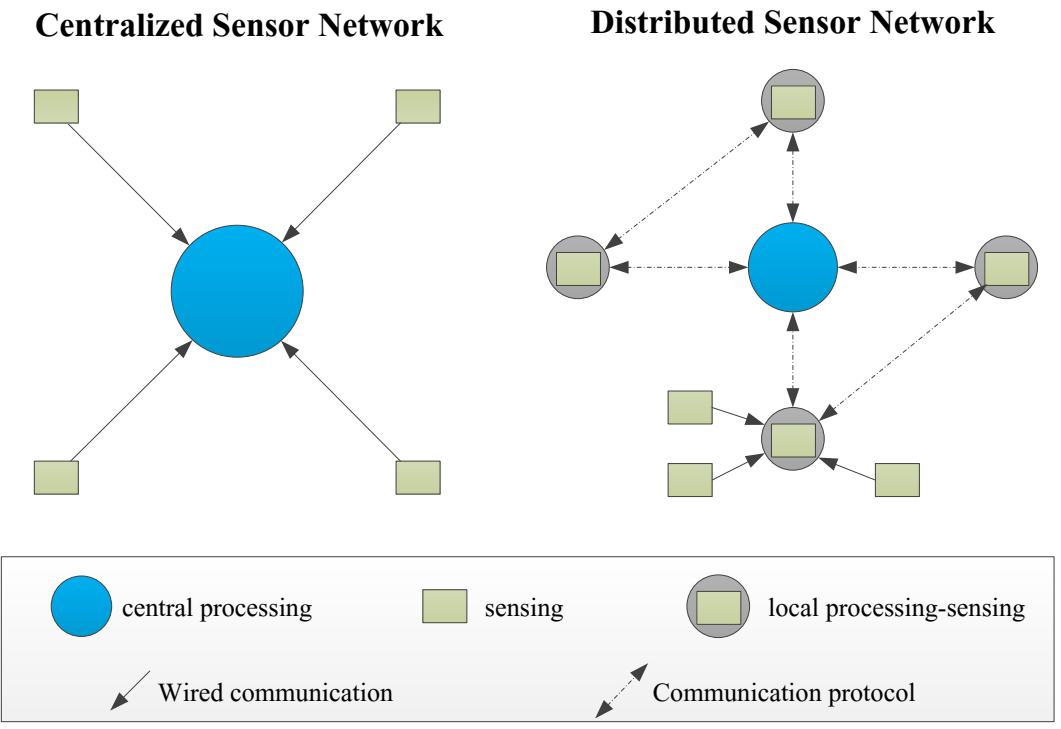

Figure 7.5: Centralised and distributed sensor networks.

This type of IoT-based monitoring systems enables the analysis of greater amounts of distributed data by carrying out efficient in situ pre-processing, facilitating the analysis of high sampling frequency signals as in the bearing case studies. The coordination of the network and the communication with the control and supervision systems is enabled by highly synchronised (wireless) communication protocols. 
Such coordination is critical to ensuring a reliable picture of the load distribution in variable operational environment. Furthermore, embedded sensing enables the access to traditionally restricted locations such as rotating frames of rotor blade systems.

While the expectations for distributed vibration monitoring systems are high, they are not easy to realise. On the one hand, the complexity of vibration monitoring is greater than other industrial IoT applications; and on the other hand, the algorithms for in-situ assessment of the vibration signal differ from the traditional, centralised implementations. This implies that the acquisition, processing and communication of the selected vibration features must be well balanced against the data acquisition, signal processing and data reduction [120]. In other words, to reap the benefits of these technologies while balancing their penalties against traditional implementations, a lean description of the sensing requirements is necessary. This highlights the relevance of a lean definition of the algorithms and, in general, the monitoring needs from the previous FBS layers.

\subsection{Stakeholder analysis}

The requirement definition according to the framework presented requires the coordination between different stakeholders of the vibration analysis and monitoring activity. Four main stakeholders are identified for the vibration monitoring problem:

Operators and maintainers are responsible for the condition of the monitored asset. These are the main receptors of the information resulting from the vibration analysis.

Designers hold the knowledge about system functionality and the failure mechanisms for the most critical components. As original designers are seldom involved in the design of monitoring systems, their role is often replaced by specialists from the asset owners. Yet designers can benefit from learning about the vibration behaviour as feedback for the continuous improvement of their systems.

Vibration analysts decide upon the treatment of the acquired signals and their interpretation. They are also responsible for the (empirical) models of the learned vibration behaviour.

Instrumentation specialists decide upon the most suitable implementation given the monitoring requirements. In the case of distributed sensors networks this category involves specialists from the different supporting technologies.

Additionally, four information flows are identified for the requirement definition 
of the monitoring strategy and implementation. These include $(A)$ the identification of monitoring needs, $(B)$ the characterisation of the failure process, $(C)$ the definition of vibration features, and $(D)$ the instruments implementation. These are illustrated in Figure 7.6. The following sections discuss these steps in detail.
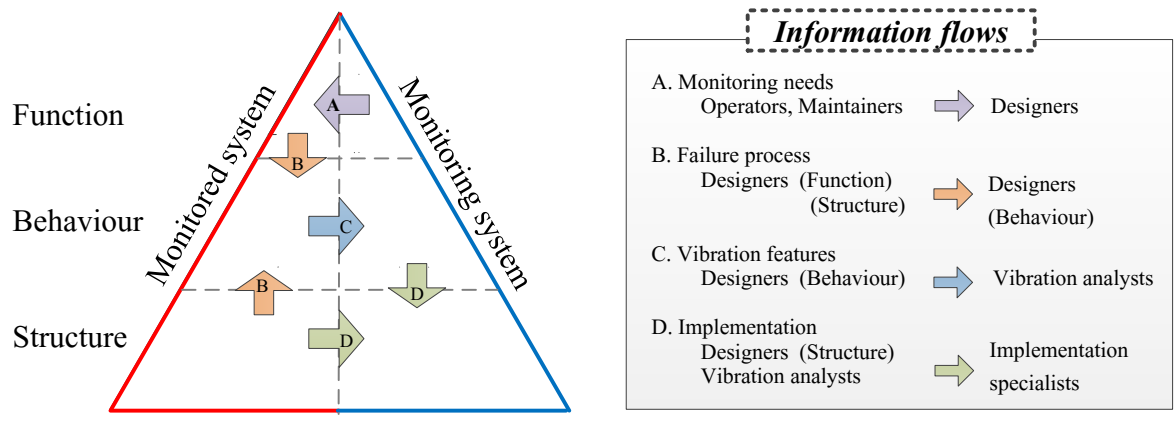

Figure 7.6: Information exchange between stakeholders of the design process for vibration monitoring systems.

\subsubsection{Monitoring strategy}

The choice of a monitoring strategy takes account of the main risks of the system based on its intrinsic degradation and the mitigation measures in place. This means that a monitoring strategy is decided based on the available knowledge of the deterioration processes and the specific goals based on the capacity of maintenance interventions.

Figure 7.7 illustrates performance and reliability as the main goals of the maintenance strategy, followed by the assessment of the system degradation. From these considerations, the resulting monitoring strategy responds to a global assessment or rather to a component-level scale; and to whether the features contribute to an immediate assessment or they are part of an accumulated diagnostics.

Four types of monitoring strategies are identified:

- Event detection is a continuous surveillance mode that focuses on identifying the existence of a damage due to transient loads, such as impacts.

- Performance monitoring focuses on the change in the operational forces considering the variation in the operational environment.

- Integrity assessment is a more elaborated interrogation strategy that assesses the changes in the main structural resonances. 


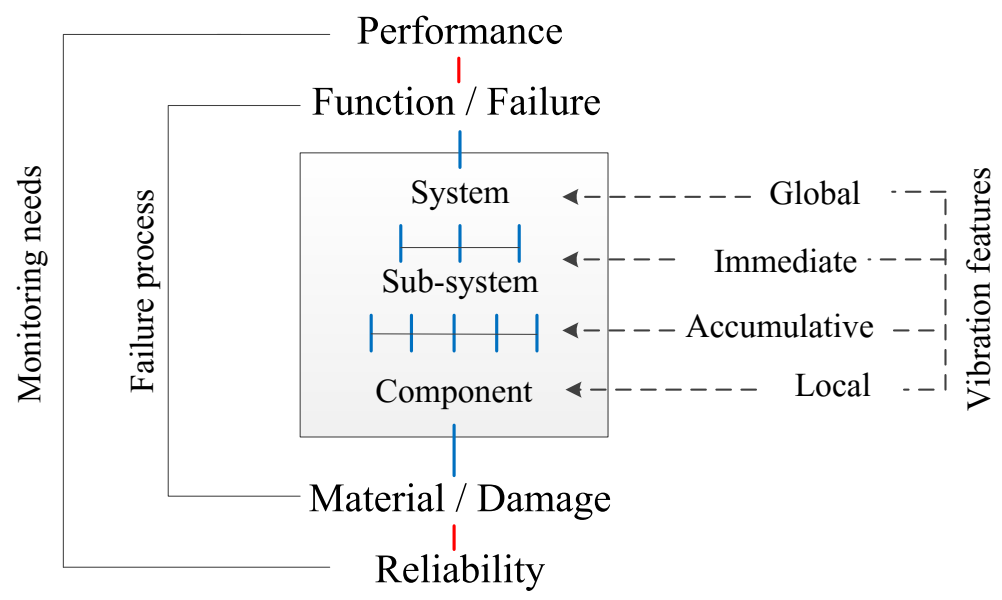

Figure 7.7: Definition of monitoring strategies.

- Damage accumulation applies to the consumption of the load-carrying capacity based on the estimation of the actual component loading history.

Designers hold the knowledge of the relation between failure process and the system functionality. This knowledge can be derived from physics-based or empirical sources. In both cases, the models must integrate the functional and structural aspects of the monitoring system (what, when and where questions) into the expected failure behaviour (how-qualitatively question). Without these models the monitoring activity becomes based merely on trial and error.

\subsubsection{Monitoring implementation}

The features defined for the monitoring strategy are the starting point of the actual implementation of the monitoring system. Multiple guidelines exist for the implementation of centralised monitoring systems for specific systems [121,122]. The case of decentralised, distributed systems demands a more tailored implementation based on the specific characteristics of the monitored object. Specifically, the features' spatial (global vs. local) and temporal (immediate vs. accumulative) aspects must be evaluated with respect to sensing, processing, communication and energy aspects of the sensor network, as shown in Figure 7.8. 


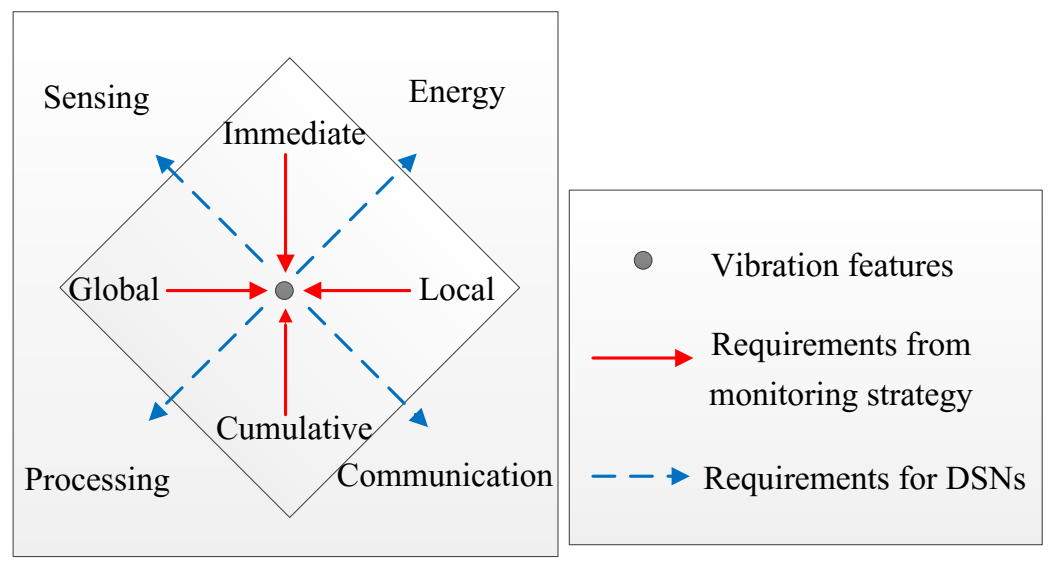

Figure 7.8: Definition of monitoring implementation.

As a general rule, the implementation of the monitoring system must abide by the simple, smart, and small design principles. The simplicity focuses on adapting the vibration algorithms for local pre-processing. The smartness considers strategies for optimising resources while performing the acquisition, processing and communication tasks. Finally, the 'small' feature refers to the space constraints that are faced by the monitored system.

\subsection{Conclusions}

In this chapter a design framework for vibration monitoring systems has been proposed. The framework is based on a structured and integrated assessment of the characteristics of both the monitored system and the monitoring system, following the systems engineering oriented Function-Behaviour-Structure (FBS) approach. The main conclusions related to the proposed framework are:

- An understanding of how the damage influences functionality is necessary for monitoring the deterioration of a mechanical system.

- The definition of a monitoring strategy must follow from the risks associated with failure proliferation and the capacity to mitigate these through maintenance interventions. 
- The interpretation of the monitored vibration response must be supported by a preliminary estimation of the most likely changes in the dynamic response and their interactions.

- Increased capabilities for register the spatial and temporal aspects of the load distribution can be achieved through distributed sensor networks, yet these require a lean requirement definition.

By following this approach, the designer is guided along the crucial steps in the development, leading to an a-priori motivation and definition of the monitoring strategy and implementation. 


\section{Chapter 8}

\section{Conclusions and Recommendations}

This dissertation presented a theoretical contribution on the study of failure in rotating mechanical systems. The contribution comprises a conceptual part oriented to understanding the failure phenomenon, based on complexity theory, and a practical part for the observation of failure, supported by a functional approach to vibration analysis.

Three different types of failure were identified: simple failures originating in lower than expected material/component capacity, complicated failures due to the simultaneous acceleration of system components due to operational environment, and complex failures which are characterised by changes in the load distribution. The complex failure category is of great concern as it involves the most uncertainties about system functionality. This is because altered load distributions derive on heterogeneous - hence more difficult to predict - deterioration of the system components. This increases the risk of severe integrity damages, as seen in catastrophic failures.

In general, complex failures cannot be attributed to a single component damage or to the operational environment alone. Rather they emerge as a combination of factors that undermine system functionality altogether. This is why they are also more difficult to address and can lead to latent problems such as chronic component replacement and under-performance.

It was shown that current approaches to failure assessment are insufficient to deal with complex failure. Material degradation models can predict the capacity 
consumption based on known failure mechanisms, while empirical models can predict system deterioration for repetitive failure events. However, these approaches do not consider the interaction of failure mechanisms or failure modes over different system components.

The complexity approach proposed in this thesis is based on two aspects of the failure phenomenon. Firstly, the designed complexity of the failing system. This means that the likelihood to develop complex failure is proportional to the interdependencies of the system. Secondly, the proposed approach recognises an emergent complexity in the system behaviour. This means that the behaviours adopted by the failing system cannot be fully predicted as they obey new reorganisation principles that are different from the design principles. Nevertheless, such emerging complexity is studied by learning through observation.

A functional approach to the observation of the failure behaviour through vibration analysis was proposed. This approach differs from the current focus on the physical aspects of the damage of some critical components such as bearings and gears. Instead, the functional approach for vibration analysis enables the comprehensive monitoring of the system-level load distribution changes and thus, facilitates the interpretation of how failure proliferates beyond the component level.

The combination of approaches, failure complexity and functional vibration analysis, was guided by the following research questions:

1. What are the mechanisms that explain complex failures?

2. What are the principles that predict the complex failure dynamics?

3. What are the features of the vibration response that enable the interpretation of load distribution changes associated with complex failure?

4. What are the requirements for the design of vibration monitoring systems for the registration of load distribution changes?

Following sections summarise the answers to these questions.

\subsection{Understanding the mechanisms of complex failure: the why}

The first unknown to resolve in relation to complex failure is why it develops despite design efforts. It was shown that a side effect of increased functional integration is the associated risk of un-identified negative interactions between the functional requirements. At the intra-component level these interactions involve failure mechanisms and functional parameters (Section 2.1.2) yielding to lower-than-expected ma- 
terial capacity. While for some components the end of the designed capacity leads to a system shut down, for others it means that the functions can be carried on suboptimally. The subsequent material degradation becomes even more detrimental to the related functions. At the inter-component level the increased functional integration leads a greater number of functions affected after component damage, but also to stronger effects (Section 2.1.3).

The problem of load distribution changes derived from component degradation was addressed from a novel perspective. Firstly, by discriminating component functional requirements as performance and reliability types, and secondly by identifying the designed dependencies among them. Reliability parameters relate to the intrinsic material capacity, thus they are conditioned by the developing failure mechanism. Performance parameters relate to the interaction between components to achieve system functionality, hence they are directly associated with the overall load distribution. Each performance parameter is supported by a number of reliability parameters, which ensure that the component function can be executed within limited tolerances for the designed component life cycle. Conversely, multiple performance parameters can depend on one single reliability parameter.

In the designed scenario, performance and reliability parameters exhibit restricted linear dependency, i.e. limited impact of consumption of the material capacity on the performance indicators. Failure induces and exacerbates the dependency between reliability and performance parameters as their relation becomes nonlinear. This means that change in load distribution - and complex failure - occurs as result of a growing number of performance parameters disrupted by material degradation beyond the designed expectations.

\subsection{Understanding the dynamics of complex failure: the how}

Altered load distributions present a conundrum for the prediction of further failure development. Along with the first significant load distribution changes, the system degradation patterns change from the designed homogeneous to an unexpected heterogeneous path. This means that given the different-than-designed loading conditions, some components will reach the end of their capacity faster, and with that new changes in the load distribution appear. Similarly the altered load distribution decreases the predictability of the component life consumption given for varying operational environment. As consequence, the available designed knowledge (i.e. PoF models) results inadequate for predicting material capacity consumption given the increased functional interdependencies and the altered load distribution. Nei- 
ther empirical models built on previous failure experiences can deliver robust and generalisable correlations about the failure proliferation.

The question of how failure proliferates has been addressed by regarding failure as a divergent and progressive transformation of the system behaviour, away from the designed state and driven by 'organising' principles different from the design logic. The three proposed principles of failure are:

Loss Energy Principle Failure increases the system entropy by reducing the effectiveness of the functional energy transformations while accelerating the system degradation.

Kinetic Energy Principle Failure evolution depends on the destabilisation effect of kinetic energy with respect to the compromised functions, the physical damage characteristics and the external environment variability. Consequently, the likelihood of predicting failure evolution is conditioned by the uncertainties associated with each of these factors.

Mode-State Dependency Principle The likelihood of an incoming failure mode (perturbation) triggering a new failure state increases with the actual system complexity (interdependency).

\subsection{Interpreting vibration behaviour: the method}

Vibration behaviour reflects the load distribution changes associated with complex failure. The vibration response directly reflects the destabilising lateral forces that decreased power transference efficiency while revealing the changes in the structural configuration as consequence of the accelerated, abnormal system degradation. Thus, interpreting the vibration response helps to understand the increase of system entropy.

A functional approach was proposed to the selection of insightful vibration features. This perspective deviates from the current emphasis on isolating the most representative features of the (physical) damages. Instead, the proposed functional approach aims at a comprehensive description of the vibration behaviour with respect to its elements and the nonlinear interactions amongst them. Two different sources of abnormal vibration behaviour were identified: the modified operational forces and structural resonances; and the damage-induced forces and local resonances. The nonlinear interactions were classified as harmonic disruption, amplitude and frequency modulation, and resonance (as a state).

The functional approach was exemplified on two case studies. The bearing cases illustrated the relevance of considering the system functionality and operation envi- 
ronment for the assessment of the component failure (structural support function). Additionally it was found that despite referring to the same component type, the three investigated systems (i.e. lab set-up, wind turbine generator and train axle box) displayed different load distributions as result of the bearing failure. Furthermore, the rotor blade case, developed through a system demonstrator illustrated the increased information potential when extending failure assessment to include multiple failure modes and not only those related to the blade-like tested beam directly.

In sum, the functional approach for vibration analysis provides significant opportunities for the construction of more insightful empirical models of the failure behaviour. Four desired attributes of the vibration features must be addressed:

(i) to relate to the system functionality,

(ii) to be robust for distinguishing between operational and damage events,

(iii) to integrate multiple aspects of the dynamic behaviour, and

(iv) to be suitable (efficient) for incorporation in the sensor system.

The feature efficiency is highly subjected to the type of monitoring implementation required as topic of the next question.

\subsection{Observing complex failure: the instruments}

The cases on roller bearings and rotor-bade systems highlighted the relevance of accurate register of the temporal and spatial aspects of the load distribution. For the case of roller bearings, the adequate signal acquisition and analysis resulted key for understanding the influence of the operational environment variability on the vibration response. For the rotor blade case, the on-blade monitoring enabled a leap on the analysis of the dynamic behaviour even with a limited number of sensors. These examples showed the need for tailoring the requirements of the vibration monitoring system according with the expected failure behaviour.

A design framework based on the Functional-Behaviour-Structure systems engineering method was proposed to address the requirements definition for vibration monitoring systems. This framework exposes the requirements from the different stakeholders related to the monitored and monitoring systems. An implementation of the framework is still to be realised, but it provides a common ground for different disciplines to discuss the integrated design of these systems. 


\subsection{Recommendations}

Given the conclusions drawn at this point, further research attention recommended to the avoiding or limiting failure is discussed next.

\subsubsection{Designing leaner mechanical systems}

The proposed concepts about the emergence of complex failure offer important opportunities for the development of leaner mechanical systems. By leaner designs it is implied to preserve or improve functionality standards while reducing material use and ensuring system reliability. Currently the detail design phase is based on the independent component failure assumption and on industry-adopted safety factors. Through the proposed concepts, systems and components can be designed considering their sensitivity to complex failure:

- which performance parameters are the most sensitive to variations of the supporting reliability parameters, and

- how such changes are likely to be reflected in the system load distribution quantitatively and qualitatively.

Sensitivity analyses are recommended for predicting the nonlinear interaction between performance and reliability interactions. These analyses could increase the knowledge about how the system changes its behaviour. Furthermore, this type of analysis can also contribute to the modelling of escalating failures, as they can quantify the degree of affectation between failure modes as discussed in Section 2.1.3.

\subsubsection{Functionality evaluation}

An integrated approach for the comprehensive evaluation of system functionality based on the proposed concepts is recommended. Such integrated functionality assessment must link material degradation models, functional models, and statistical models for evaluating different aspects of the failure behaviour. For instance:

- updated damage models for the monitoring (accounting) of material capacity consumption for the actual load distribution monitored.

- functional models that evaluate the updated risks of failure proliferation based on known designed dependency,

- statistical models of predicted variations on the operational environment and system expected use. 
These models must be complemented with the vibration monitoring data and other sources that describe different aspects of the failure behaviour (e.g. alarm logs, lube oil analysis). The formulated principles provide a guideline for the interpretation and prediction of the failure behaviour. Thus, the model goal becomes to characterise and predict the divergence and progressivism character of failure. This means that the modelling effort is directed to the quantitative and qualitative description of the system behaviour at specific failure states. Also the prognostics efforts are directed to estimate the updated risk given an ongoing degradation. This combination aims to bridge the physics-based and empirical approaches to failure assessment.

\subsubsection{Distributed sensor networks}

Distributed monitoring systems promise greater autonomy at the measurement location for the registration and preliminary assessment of the vibration behaviour. This autonomy is supported by the integration of different technologies, such as embedded processing and wireless communication. However, to harvest the potential of distributed monitoring systems a lean definition of the design requirements is needed. The adoption of these technologies for vibration monitoring systems requires more specific guidelines to be developed. These must address suitability of the different options within sensing technologies, wireless communication, local processing and energy harvesting with respect to specific monitoring strategies.

\subsection{Final remarks}

Although failure is unlikely to be avoided completely, it is possible to limit its worst effects. Vibration analysis is a valuable diagnostics tool as it reflects the changes in the system dynamic behaviour even before actual damage occurs. Yet this requires an approach for learning from the machines rather than just machine learning. This entails anticipating the systems' emerging interdependencies and uncertainties based on the effective integration of the various sources of knowledge available and implementing tailored monitoring strategies supported by intelligent sensing networks. The effective combination of these aspects represents a step towards the development of leaner yet more reliable machines and, consequently, towards a greener and more sustainable future. 


\section{References}

[1] IRENA. Accelerating the Energy Transition through Innovation, a working paper based on global REmap analysis. 2017.

[2] Taesik Lee. Complexity Theory in Axiomatic Design. page 182, 2003.

[3] Nam Pyo Suh. Complexity in Engineering. CIRP Annals - Manufacturing Technology, 54(2):46-63, 2005.

[4] Tomer Ben-Yehuda, Avigdor Zonnenshain, and Reuven Katz. Evaluating complicatedness in mechanical design. Procedia CIRP, 36:41-47, 2015.

[5] E. Zio. Reliability engineering: Old problems and new challenges. Reliability Engineering E System Safety, 94(2):125-141, feb 2009.

[6] D Valis and L M Bartlett. The Failure Phenomenon. International Journal of Performability Engineering, 6(2):181-190, 2010.

[7] Iain Staffell and Richard Green. How does wind farm performance decline with age? Renewable Energy, 66:775-786, jun 2014.

[8] Tiedo Tinga. Application of physical failure models to enable usage and load based maintenance. Reliability Engineering and System Safety, 95(10):1061-1075, 2010.

[9] Seongwoo Woo. Parametric Accelerated Life Testing in Mechanical/Civil System. In Reliability Design of Mechanical Systems, pages 171-219. Springer International Publishing, Cham, 2017.

[10] Enrico Zio and Nicola Pedroni. Uncertainty characterization in risk analysis for decision-making practice. 2012.

[11] Michael Pecht and Jie Gu. Pecht, M., \& Jie Gu. (2009). Physics-of-failure-based prognostics for electronic products. Transactions of the Institute of Measurement and Control, 2009.

[12] Tiedo Tinga. Predictive Maintenance of Military Systems Based on Physical Failure Models. Chemical Engineering Transactions, 33, 2013.

[13] Prashanth Pillai, Anshul Kaushik, Shivanand Bhavikatti, Arjun Roy, and Virendra Kumar. A Hybrid Approach for Fusing Physics and Data for Failure Prediction. International Journal of Prognostics and Health Management, pages 2153-2648, 2016. 
[14] Hongyu Yang, Joseph Mathew, and Lin Ma. Vibration Feature Extraction Techniques for Fault Diagnosis of Rotating Machinery -A Literature Survey. In Asia Pacific Vibration Conference, Gold Coast, Australia., 2003.

[15] Jérôme Antoni and R.B. Randall. The spectral kurtosis: application to the vibratory surveillance and diagnostics of rotating machines. Mechanical Systems and Signal Processing, 20(2):308-331, 2006.

[16] Robert Bond Randall. Wiley: Vibration-based Condition Monitoring: Industrial, Aerospace and Automotive Applications - Robert Bond Randall. Wiley, 2011.

[17] Wade A. Smith and Robert B. Randall. Rolling element bearing diagnostics using the Case Western Reserve University data: A benchmark study. Mechanical Systems and Signal Processing, 64-65:100-131, dec 2015.

[18] G Cybenkot. Approximation by superpositions of a sigmoidal function. Math. Control Signals Systems, 2:303-314, 1989.

[19] Gaetan Kerschen, Keith Worden, Alexander F Vakakis, and Jean-Claude Golinval. Past, present and future of nonlinear system identification in structural dynamics. Mechanical Systems and Signal Processing 2, 20(2006):505-592, 2005.

[20] Aiwina Heng, Andy C.C. Tan, Joseph Mathew, Neil Montgomery, Dragan Banjevic, and Andrew K.S. Jardine. Intelligent condition-based prediction of machinery reliability. Mechanical Systems and Signal Processing, 23(5):1600-1614, jul 2009.

[21] Andrew K.S. Jardine, Daming Lin, and Dragan Banjevic. A review on machinery diagnostics and prognostics implementing condition-based maintenance. Mechanical Systems and Signal Processing, 20(7):1483-1510, oct 2006.

[22] Feng Jia, Yaguo Lei, Jing Lin, Xin Zhou, and Na Lu. Deep neural networks: A promising tool for fault characteristic mining and intelligent diagnosis of rotating machinery with massive data. Mechanical Systems and Signal Processing, 72:303-315, 2016.

[23] Jay Lee, Fangji Wu, Wenyu Zhao, Masoud Ghaffari, Linxia Liao, and David Siegel. Prognostics and health management design for rotary machinery systems - Reviews, methodology and applications. Mechanical Systems and Signal Processing, 42(1-2):314334, jan 2014.

[24] Keith Worden, Wieslaw J. Staszewski, and James J. Hensman. Natural computing for mechanical systems research: A tutorial overview. Mechanical Systems and Signal Processing, 25(1):4-111, jan 2011.

[25] Feng Jia, Yaguo Lei, Jing Lin, Xin Zhou, and Na Lu. Deep neural networks: A promising tool for fault characteristic mining and intelligent diagnosis of rotating machinery with massive data. Mechanical Systems and Signal Processing, 72-73:303-315, may 2016.

[26] Wolfgang Pietsch. Aspects of theory - ladenness in data - intensive science. Philosophy of Science, 82(5):905-916, 2015.

[27] Venkat Venkatasubramanian and Raghunathan Rengaswamy. A review of process fault detection and diagnosis Part I : Quantitative model-based methods. Computers $\mathcal{E}$ chemical ..., 27:293-311, 2003. 
[28] Venkat Venkatasubramanian, Raghunathan Rengaswamy, and Surya N Kavuri. A review of process fault detection and diagnosis. Computers $\mathcal{E}$ Chemical Engineering, 27(3):313-326, mar 2003.

[29] a. Hajnayeb, a. Ghasemloonia, S.E. Khadem, and M.H. Moradi. Application and comparison of an ANN-based feature selection method and the genetic algorithm in gearbox fault diagnosis. Expert Systems with Applications, 38(8):10205-10209, aug 2011.

[30] Jay Lee, Jun Ni, Dragan Djurdjanovic, Hai Qiu, and Haitao Liao. Intelligent prognostics tools and e-maintenance. Computers in Industry, 57(6):476-489, aug 2006.

[31] Aiwina Heng, Sheng Zhang, Andy C.C. Tan, and Joseph Mathew. Rotating machinery prognostics: State of the art, challenges and opportunities. Mechanical Systems and Signal Processing, 23(3):724-739, apr 2009.

[32] Yaguo Lei, Naipeng Li, Liang Guo, Ningbo Li, Tao Yan, and Jing Lin. Machinery health prognostics: A systematic review from data acquisition to RUL prediction. Mechanical Systems and Signal Processing, 104:799-834, may 2018.

[33] Ying Peng, Ming Dong, and Ming Jian Zuo. Current status of machine prognostics in condition-based maintenance: a review. The International Journal of Advanced Manufacturing Technology, 50(1-4):297-313, jan 2010.

[34] Xiao Sheng Si, Wenbin Wang, Chang Hua Hu, and Dong Hua Zhou. Remaining useful life estimation - A review on the statistical data driven approaches. European Journal of Operational Research, 213(1):1-14, 2011.

[35] J.Z. Sikorska, M. Hodkiewicz, and L. Ma. Prognostic modelling options for remaining useful life estimation by industry. Mechanical Systems and Signal Processing, 25(5):18031836, jul 2011.

[36] Sarah A. Sheard. Practical Applications of Complexity Theory for Systems Engineers. INCOSE International Symposium, 15(1):923-939, jul 2005.

[37] J.S. Gero. Design Prototypes: A Knowledge Representation Schema for Design. AI Magazine, 11(4):26, 1990.

[38] John S. Gero and Udo Kannengiesser. A functionbehaviorstructure ontology of processes. Ai Edam, 21(04):379-391, sep 2007.

[39] Peter. Erdi. Complexity explained. Springer, 2008.

[40] Yichun Zhou. Micro- and Macromechanical Properties of Materials. 2013.

[41] Abhijit Dasgupta and Michael Pecht. Material failure mechanisms and damage models. IEEE Transactions on Reliability, 40(5):531-536, 1991.

[42] Tiedo Tinga. Principles of Loads and Failure Mechanisms. Springer Series in Reliability Engineering. Springer London, London, 2013.

[43] Tiedo Tinga. Mechanism based failure analysis. 2012.

[44] Zhiguo Zeng, Yunxia Chen, Enrico Zio, and Rui Kang. A compositional method to model dependent failure behavior based on PoF models. Chinese Journal of Aeronautics, 30(5):1729-1739, 2017. 
[45] Jaap Schijve. Fatigue of Structures and Materials. Springer Netherlands, Dordrecht, 2009.

[46] Ying Chen, Liu Yang, Cui Ye, and Rui Kang. Failure mechanism dependence and reliability evaluation of non-repairable system. Reliability Engineering \& System Safety, 138:273-283, jun 2015.

[47] Zhiguo Zeng, Rui Kang, and Yunxia Chen. Using PoF models to predict system reliability considering failure collaboration. Chinese Journal of Aeronautics, 29(5):1294-1301, oct 2016.

[48] Chun yang Li, Xun Chen, Xiao shan Yi, and Jun yong Tao. Heterogeneous redundancy optimization for multi-state series-parallel systems subject to common cause failures. Reliability Engineering and System Safety, 2010.

[49] Jose E. Ramirez-Marquez and David W. Coit. Optimization of system reliability in the presence of common cause failures. Reliability Engineering and System Safety, 2007.

[50] Michael Van Eeten, Albert Nieuwenhuijs, Eric Luijf, Marieke Klaver, and Edite Cruz. The state and the threat of cascading failure across critical infrastructure: The implications of empirical evidence from media incident reports. Public Administration, 89(2):381-400, jun 2011.

[51] Yihai Zhu, Jun Yan, Yan Sun, and Haibo He. Revealing Cascading Failure Vulnerability in Power Grids Using Risk-Graph. IEEE Transactions on Parallel and Distributed Systems, 25(12):3274-3284, dec 2014.

[52] Panayiotis Kotzanikolaou, Marianthi Theoharidou, and Dimitris Gritzalis. Cascading Effects of Common-Cause Failures in Critical Infrastructures. In Jonathan Butts and Sujeet Shenoi, editors, Critical Infrastructure Protection VII, chapter 12, pages 171-182. Springer Berlin Heidelberg, Berlin, Heidelberg, 2013.

[53] N.C. Caballé, I.T. Castro, C.J. Pérez, and J.M. Lanza-Gutiérrez. A condition-based maintenance of a dependent degradation-threshold-shock model in a system with multiple degradation processes. Reliability Engineering \& System Safety, 134:98-109, feb 2015.

[54] Ali Mosleh. Common cause failures: An analysis methodology and examples. Reliability Engineering \& System Safety, 34(3):249-292, jan 1991.

[55] Adriaan Van Horenbeek and Liliane Pintelon. A dynamic predictive maintenance policy for complex multi-component systems. Reliability Engineering \& System Safety, 120:3950, dec 2013.

[56] Qingyuan Zhang, Rui Kang, and Meilin Wen. Belief Reliability for Uncertain Random Systems. IEEE Transactions on Fuzzy Systems, 26(6):3605-3614, dec 2018.

[57] Leonardo Dueñas-Osorio and Srivishnu Mohan Vemuru. Cascading failures in complex infrastructure systems. Structural Safety, 31(2):157-167, mar 2009.

[58] Isaac Hernandez-Fajardo and Leonardo Dueñas-Osorio. Probabilistic study of cascading failures in complex interdependent lifeline systems. Reliability Engineering and System Safety, 2013. 
[59] Mengkai Xu, Xiaoning Jin, Sagar Kamarthi, and Md. Noor-E-Alam. A failuredependency modeling and state discretization approach for condition-based maintenance optimization of multi-component systems. Journal of Manufacturing Systems, 47:141-152, apr 2018.

[60] Li Yang, Xiaobing Ma, and Yu Zhao. A condition-based maintenance model for a threestate system subject to degradation and environmental shocks. Computers $\mathcal{E}$ Industrial Engineering, 105:210-222, mar 2017.

[61] Linkan Bian and Nagi Gebraeel. Stochastic modeling and real-time prognostics for multi-component systems with degradation rate interactions. IIE Transactions, 46(5):470-482, may 2014.

[62] Zhiguo Zeng and Enrico Zio. Dynamic Risk Assessment Based on Statistical Failure Data and Condition-Monitoring Degradation Data. IEEE Transactions on Reliability, 67(2):609-622, jun 2018.

[63] Prashant M. Pawar and Ranjan Ganguli. On the effect of progressive damage on composite helicopter rotor system behavior. Composite Structures, 78(3):410-423, may 2007.

[64] Herman Soemers. Design Principles for Precision Mechanisms. T-Point Print VoF, Enschede, 2010.

[65] Behnam Ghalamchi, Sopanen Jussi, and Aki Mikkola. Simple and Versatile Dynamic Model of Spherical Roller Bearing, 2013.

[66] Alireza Moazenahmadi, Dick Petersen, and Carl Howard. A nonlinear dynamic model of the vibration response of defective rolling element bearings. (November), 2013.

[67] A. Soleimani and S.E. Khadem. Early fault detection of rotating machinery through chaotic vibration feature extraction of experimental data sets. Chaos, Solitons $\mathcal{E}$ Fractals, 78:61-75, sep 2015.

[68] M. Li and L. He. The dynamics of a parallel-misaligned and unbalanced rotor system under the action of non-linear oil film forces. Proceedings of the Institution of Mechanical Engineers, Part C: Journal of Mechanical Engineering Science, 224(9):1875-1889, 2010.

[69] Zhaoyi Guan, Peng Chen, Xiaoyu Zhang, Xiong Zhou, and Ke Li. Vibration analysis of shaft misalignment and diagnosis method of structure faults for rotating machinery. International Journal of Performability Engineering, 13(4):337-347, 2017.

[70] Xiaowang Chen and Zhipeng Feng. Time-frequency analysis of torsional vibration signals in resonance region for planetary gearbox fault diagnosis under variable speed conditions. IEEE Access, 5:21918-21926, 2017.

[71] Fakher Chaari, Walter Bartelmus, Radoslaw Zimroz, Tahar Fakhfakh, and Mohamed Haddar. Gearbox vibration signal amplitude and frequency modulation, jan 2012.

[72] Johann Lenz and Fikre E Boru. Torsional Vibration Problem in Reciprocating Compressor Case Study. SIRM 2015- 11th International Conference on Vibrations in Rotating Machines, (February):1-11, 2015. 
[73] Zeki Kral and Hira Karagülle. Vibration analysis of rolling element bearings with various defects under the action of an unbalanced force. Mechanical Systems and Signal Processing, 20(8):1967-1991, nov 2006.

[74] K. Mori, N. Kasashima, T. Yoshioka, and Y. Ueno. Prediction of spalling on a ball bearing by applying the discrete wavelet transform to vibration signals. Wear, 195(1-2):162-168, 1996.

[75] Robert B. Randall and Jérôme Antoni. Rolling element bearing diagnosticsA tutorial. Mechanical Systems and Signal Processing, 25(2):485-520, feb 2011.

[76] Robert Randall and Wade Smith. New cepstral methods for the diagnostics of gear and bearing faults under variable speed conditions. In ICSV 23 Conference, Athens, 2016.

[77] Sarabjeet Singh, Uwe G. Köpke, Carl Q. Howard, and Dick Petersen. Analyses of contact forces and vibration response for a defective rolling element bearing using an explicit dynamics finite element model. Journal of Sound and Vibration, 333(21):5356-5377, oct 2014.

[78] Sarabjeet Singh, Carl Q. Howard, and Colin H. Hansen. An extensive review of vibration modelling of rolling element bearings with localised and extended defects. Journal of Sound and Vibration, 357:300-330, jun 2015.

[79] N Tandon and a Choudhury. A review of vibration and acoustic measurement methods for the detection of defects in rolling element bearings. Tribology International, 32(8):469480, 1999.

[80] Ruonan Liu, Boyuan Yang, Enrico Zio, and Xuefeng Chen. Artificial intelligence for fault diagnosis of rotating machinery: A review. Mechanical Systems and Signal Processing, 108:33-47, aug 2018.

[81] Piet M. Lugt. A Review on Grease Lubrication in Rolling Bearings. Tribology Transactions, 52(4):470-480, jun 2009.

[82] Y.H. Wijnant, J.A. Wensing, and G.C. Nijen. The influence of lubrication on the dynamic behaviour of ball bearings. Journal of Sound and Vibration, 222(4):579-596, 1999.

[83] ISO. ISO 15243 Rolling bearings Damage and failures Terms, characteristics and causes, 2009.

[84] SKF Group. Bearing damage and failure analysis. Technical report, 2017.

[85] J. E. Berry. Analysis II - Concentrated Vibration Signature Analysis and Related Condition Monitoring Technique. Technical Associates of Charlotte, Charlotte, 2nd edition, 1997.

[86] A. Sanchez Ramirez, R. Loendersloot, T. Tinga, and G. D'Angelo. Impact response characterization as basis for bearing diagnostics and prognostics. In Cham Springer, editor, Proceedings of the 9th IFToMM International Conference on Rotor Dynamics. Mechanisms and Machine Science, vol 21. Springer, jan 2015.

[87] Behnam Ghalamchi, Jussi Sopanen, and Aki Mikkola. Modeling and Dynamic Analysis of Spherical Roller Bearing with Localized Defects: Analytical Formulation to Calculate Defect Depth and Stiffness. Shock and Vibration, 2016:1-11, jan 2016. 
[88] Alireza Moazen Ahmadi, Dick Petersen, and Carl Howard. A nonlinear dynamic vibration model of defective bearings The importance of modelling the finite size of rolling elements. Mechanical Systems and Signal Processing, 52-53:309-326, feb 2015.

[89] Alessandro Cisi, Alessandro Zanella, and Giuseppe D Angelo. Rolling Element Bearing Fault Detection Based on Orthogonal Hilbert-Huang Transform. CM 2013 and MFPT 2013, (Cm), 2013.

[90] Jaouher Ben Ali, Nader Fnaiech, Lotfi Saidi, Brigitte Chebel-Morello, and Farhat Fnaiech. Application of empirical mode decomposition and artificial neural network for automatic bearing fault diagnosis based on vibration signals. Applied Acoustics, 89:1627, mar 2015.

[91] D. Abboud, J. Antoni, S. Sieg-Zieba, and M. Eltabach. Envelope analysis of rotating machine vibrations in variable speed conditions: A comprehensive treatment. Mechanical Systems and Signal Processing, 84:200-226, feb 2017.

[92] J.F. Kaiser. On a simple algorithm to calculate the 'energy' of a signal. In International Conference on Acoustics, Speech, and Signal Processing, pages 381-384. IEEE, 2009.

[93] I Antoniadou, G Manson, N Dervilis, T Barszcz, W J Staszewski, and K Worden. Use of the Teager-Kaiser energy operator for condition monitoring of a wind turbine gearbox. ISMA, 2012:4255-4268, 2012.

[94] Robert Randall and Wade Smith. Use of the Teager Kaiser Energy Operator to estimate machine speed.

[95] Gerard J.W. van Bussel, Cyril Boussion, and Claudia Hofemann. A Possible Relation between Wind Conditions, Advanced Control and Early Gearbox Failures in Offshore Wind Turbines. Procedia CIRP, 11:301-304, 2013.

[96] Joel Igba, Kazem Alemzadeh, Christopher Durugbo, and Egill Thor Eiriksson. Analysing RMS and peak values of vibration signals for condition monitoring of wind turbine gearboxes. Renewable Energy, 91:90-106, 2016.

[97] Radoslaw Zimroz, Walter Bartelmus, Tomasz Barszcz, and Jacek Urbanek. Wind Turbine Main Bearing Diagnosis - A Proposal of Data Processing and Decision Making Procedure under Non Stationary Load Condition. Key Engineering Materials, 518:437-444, jul 2012.

[98] ISO. ISO 10816-21 Mechanical vibration Evaluation of machine vibration by measurements on non-rotating parts Part 21: Horizontal axis wind turbines with gearbox, 2015.

[99] A. H. Bonnett and G. C. Soukup. Cause and analysis of stator and rotor failures in three-phase squirrel-cage induction motors. IEEE Transactions on Industry Applications, 28(4):921-937, 1992.

[100] SKF-Group. Railway technical handbook, volume 2. 2012.

[101] Mani Entezami, Clive Roberts, Paul Weston, Edward Stewart, Arash Amini, and Mayorkinos Papaelias. Perspectives on railway axle bearing condition monitoring. Proceedings of the Institution of Mechanical Engineers, Part F: Journal of Rail and Rapid Transit, 234(1):17-31, jan 2020. 
[102] Rafał Burdzik and Bogusław Nowak. Identification of the Vibration Environment of Railway Infrastructure. Procedia Engineering, 187:556-561, jan 2017.

[103] I Corni, N Symonds, R J K Wood, A Wasenczuk, and D Vincent. Real-time on-board condition monitoring of train axle bearings. In The Stephenson conference: research for railways., London, 2015.

[104] D. Kim and H. Oh. EMD: A Package for Empirical Mode Decomposition and Hilbert Spectrum. The R Journal, 1(1):40-46, 2009.

[105] Gabriel Rilling, Patrick Flandrin, Paulo Gon, and De Lyon. on Empirical Mode Decomposition and Its Algorithms. IEEEEURASIP Workshop on Nonlinear Signal and Image Processing NSIP, 3:8-11, 2003.

[106] S.E. Oosterik. Towards on-blade monitoring of Rotating Blade Systems. PhD thesis, University of Twente, 2014.

[107] A.R.S. Bramwell, George Done, and David Balmford. Bramwell's Helicopter Dynamics. Bramwell's Helicopter Dynamics, pages 33-76, 2000.

[108] Prashant M. Pawar and Ranjan Ganguli. Modeling progressive damage accumulation in thin walled composite beams for rotor blade applications. Composites Science and Technology, 66(13):2337-2349, 2006.

[109] Niranjan Roy and Ranjan Ganguli. Helicopter rotor blade frequency evolution with damage growth and signal processing. Journal of Sound and Vibration, 283(3-5):821-851, may 2005.

[110] M. C. Madhar. Towards the Design of a Vibration Monitoring System for a Rotor Blade System in Operation. Technical report, 2016.

[111] Joris Wolters. Design and Construction of a demonstrator of Rotor-Blade Systems. Technical report, University of Twente, 2015.

[112] Pieter H. De Jong, Andre De Boer, Richard Loendersloot, and Peter J.M. Van Der Hoogt. Power harvesting in a helicopter rotor using a piezo stack in the lag damper. Journal of Intelligent Material Systems and Structures, 24(11):1392-1404, 2012.

[113] Dan Patt, Dennis Bernstein, Jaganath Chandrasekar, Peretz Friedmann, and Li Liu. Higher-Harmonic-Control Algorithm for Helicopter Vibration Reduction Revisited. Journal of Guidance, Control, and Dynamics, 28(5):918-930, 2005.

[114] Sanchez Ramirez, K. Das, R. Loendersloot, T. Tinga, and P. Havinga. Wireless sensor network for helicopter rotor blade vibration monitoring: Requirements definition and technological aspects, volume 569-570. 2013.

[115] E Echavarria, T Tomiyama, and G.J.W van Brussel. Fault Diagnosis approach based on a model-based reasoner and a functional designer for a wind turbine. An approach towards self-maintenance. In Journal of Physics: Conference Series, page 012078, 2007.

[116] Yoshinobu Kitamura, Masakazu Kashiwase, Masayoshi Fuse, and Riichiro Mizoguchi. Deployment of an ontological framework of functional design knowledge. Advanced Engineering Informatics, 18(2):115-127, apr 2004. 
[117] Y. Umeda and T. Tomiyama. Functional reasoning in design. IEEE Expert, 12(2):42-48, mar 1997.

[118] C.-P. Fritzen and P. Kraemer. Self-diagnosis of smart structures based on dynamical properties. Mechanical Systems and Signal Processing, 23(6):1830-1845, aug 2009.

[119] K. Romer and F. Mattern. The design space of wireless sensor networks. IEEE Wireless Communications, 11(6):54-61, 2004.

[120] Vignesh Raja Karuppiah Ramachandran, Andrea Sánchez Ramírez, Berend Jan van der Zwaag, and Paul Havinga. On-node Signal Processing to Reduce the Power Consumption of Wireless Sensor Nodes for Vibration Monitoring. In IEEE Ninth International Conference on Intelligent Sensors, Sensor Networks and Information Processing (ISSNIP),, pages 1-6, Singapore, 2014.

[121] ISO. ISO 13373 Condition Monitoring and Diagnostics of Machines - Vibration Monitoring, 2002.

[122] ISO. ISO 17359 Condition monitoring and diagnostics of machines General guidelines. Technical report, ISO, 2003.

[123] Nader Sawalhi and Robert B Randall. The application of spectral kurtosis to bearing diagnostics. (November):393-398, 2004. 


\section{Appendix A}

\section{Roller Bearings Vibration}

This appendix presents information complementary to the vibration analysis for roller bearings presented in Chapter 5 . The discussion focuses on the variations in the energy distribution, as revealed in the spectral representations, for the three cases presented. This is provided as a guidance for the selection of signal processing algorithms based on the characteristics of the signals.

As discussed in Section 5.2.3, the complexity of the vibration signals depends greatly on the changes in the elements of the vibration signal within the observation period. The stationary case corresponds to the constant magnitude and frequency content of the driving forces, as in the lab set-up case. The simplest nonstationary case occurs due to the variation of the magnitude of the driving operational forces, as in the wind turbine case. However, this does not imply that the vibration behaviour changes in magnitude only, as nonlinear phenomena can lead to new frequency components of the response (Section 4.4). Nevertheless, the expected changes in the vibration response are mainly quantitative and could be mapped to the variations in the input forces. A more complex scenario occurs when the frequency content of the driving forces changes in both magnitude and shape, as in the case of the train axle box. Consequently, the resulting variations in the vibration response will be considered as qualitative.

The appendix is organised as follows. Section A.1 presents a comparison of different approaches to the spectral analysis for the reference case (without damage) of the lab set-up case. Sections A.2 and A.3 explain the criteria for the classification of the vibration signals for, respectively, the wind turbine generator and train axle box cases. 


\section{A.1 Undamaged behaviour}

The reference case of the lab set-up is used to illustrate the expected spectral distribution in the absence of damage. The time waveform shown in Figure A.1a displays an almost random pattern. The corresponding Power Spectrum Density (PSD), Figure A.1b, shows a decreasing noise floor with some discrete modes at approximately $1000 \mathrm{~Hz}, 2000 \mathrm{~Hz}$ and $5000 \mathrm{~Hz}$. Two additional representations of the spectral distribution are used to highlight the active modes of the system. These are integral (i.e. cumulative) and derivative of the PSD, shown in Figures A.1c. and A.1d respectively.

The normalised cumulative representation illustrates how the energy of the vibration signal increases over the frequency range, as shown in Figure A.1c. For the reference case it is seen that the signal power increases moderately up to $1000 \mathrm{~Hz}$, after which the greatest rise occurs. This is followed by only one modest rise at 2000 $\mathrm{Hz}$, after which there are no further relevant increases. This example shows how the integral visualization allows a quick assessment of the relevant frequency range of the signal and its distribution., From this representation a novel feature $f_{50}$ is extracted to represent the frequency at which the signal reaches fifty per cent $(50 \%)$ power. This feature will prove useful when comparing nonstationary conditions, as will be seen in the wind turbine and train cases.

The derivative representation also illustrates distinction between the greatest disruptions in the overall noise floor of the signal, as shown in Figure A.1d. A comparison between the normal PSD spectrogram and the spectrogram based on the derivative PSD is shown in Figures A.1e and A.1f . It is seen that the derivativebased spectrogram shows greater contrast increases, which helps to identify the relevant modes clearer. The extra contrast is useful when there are too many relevant events within a close frequency range. There are multiple signal processing methods to identify ranges of active vibration activity. In particular the kurtogram is a very efficient tool for identifying frequency ranges with high kurtosis (i.e. impulsiveness) values $[15,123]$. Nevertheless, the derivative representation and the associated spectrograms are used for the sake of simplicity. 


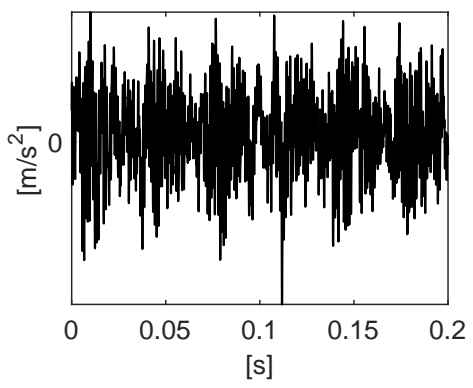

(a) Time waveform

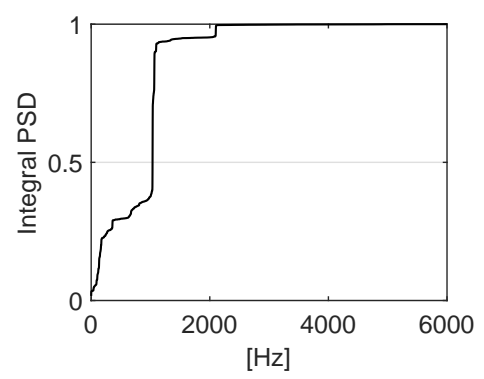

(c) Integral

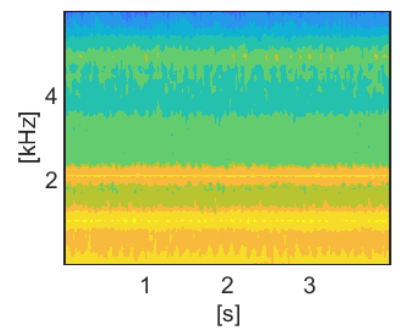

(e) PSD spectrogram

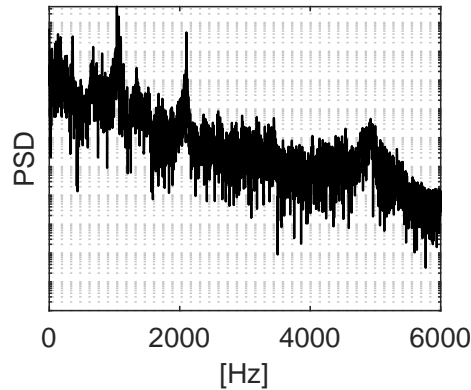

(b) PSD spectrum

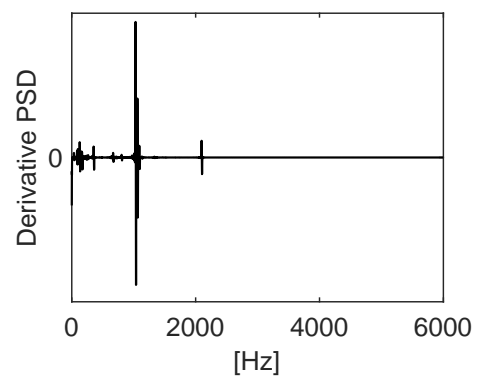

(d) Derivative

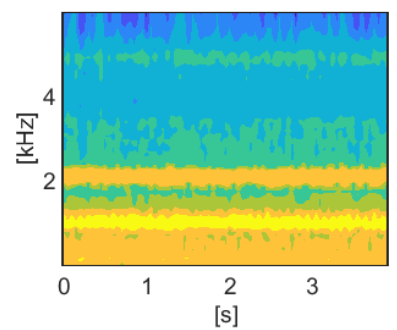

(f) Derivative-based PSD spectrogram

Figure A.1: Reference case for lab set-up case 


\section{A.2 Quantitative variations}

The variability of the vibration behaviour of wind turbine generators is attributed mostly to the magnitude changes of the operational forces. However, in the specific case presented there is a higher variability in the current output than normally expected, probably due to electrical problems. This adds additional complexity to the treatment of the vibration signal due to transients and highly nonlinear behaviour. Nevertheless, the example is very illustrative of nonstationary conditions.

A first evaluation of the correlation between the current output and the acceleration (samples of $0.4 \mathrm{~s}$ ) was performed, as seen in Figure 5.15b. This showed that the lower and higher current values led to relatively greater acceleration values. For this reason a classification of the instantaneous samples was carried out in order to analyse the behaviour at those conditions.

The classification variables are defined as follows. A nonlinear category gathers the intervals with the highest mean value $X_{\text {mean }}$. Three remaining categories are defined according to the current value $\mathbf{C}$. The classification is carried for the acceleration signal filtered between $10 \mathrm{~Hz}$ and $2000 \mathrm{~Hz} X_{10-2000}$ as recommended by the guideline for wind turbine vibration assessment VDI 3834.

- nonlinear $X_{10-2000}>X_{\text {mean }}$

- low : $X_{10-2000} \leq X_{\text {mean }} \& \mathrm{C}<=C_{\text {low }}$

- medium: $X_{10-2000} \leq X_{\text {mean }} \& C_{\text {low }}>\mathrm{C} \leq C_{\text {high }}$

- high : $X_{10-2000} \leq X_{\text {mean }} \& \mathrm{C}>C_{\text {high }}$

The classification parameters for the different signals are shown in Table A.1.

Table A.1: Classification parameters for generator bearing signals

\begin{tabular}{lccc}
\hline & $X_{\text {mean }}$ [g] & $C_{\text {low }}[\mathbf{A}]$ & $C_{\text {high }}$ [A] \\
\hline M_6 & 0.12 & 200 & 900 \\
M_10 & 0.3 & 100 & 900 \\
\hline
\end{tabular}

Figure A.2 show the classification procedure for M10. Figure A.2a presents a time signal intervals classified according to the associated current as low, medium and high current, and a nonlinear distinction for the signals with the highest mean value. The corresponding spectral distribution evaluated by the feature $f_{50}$ is shown in Figure Figure A.2b The detail of the normalised PSD cumulative for each category is presented in Figure A.3 . 


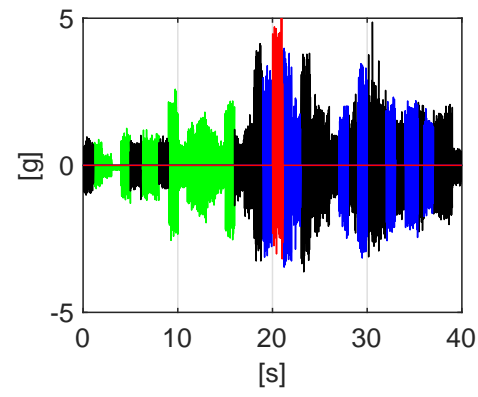

(a) Time waveform

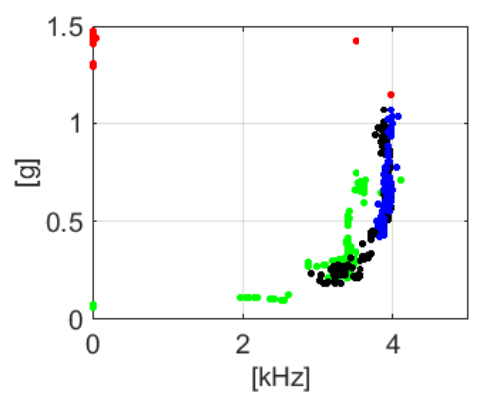

(b) $f_{50}$ versus rms value

Figure A.2: Classification of the acceleration signal intervals for M10
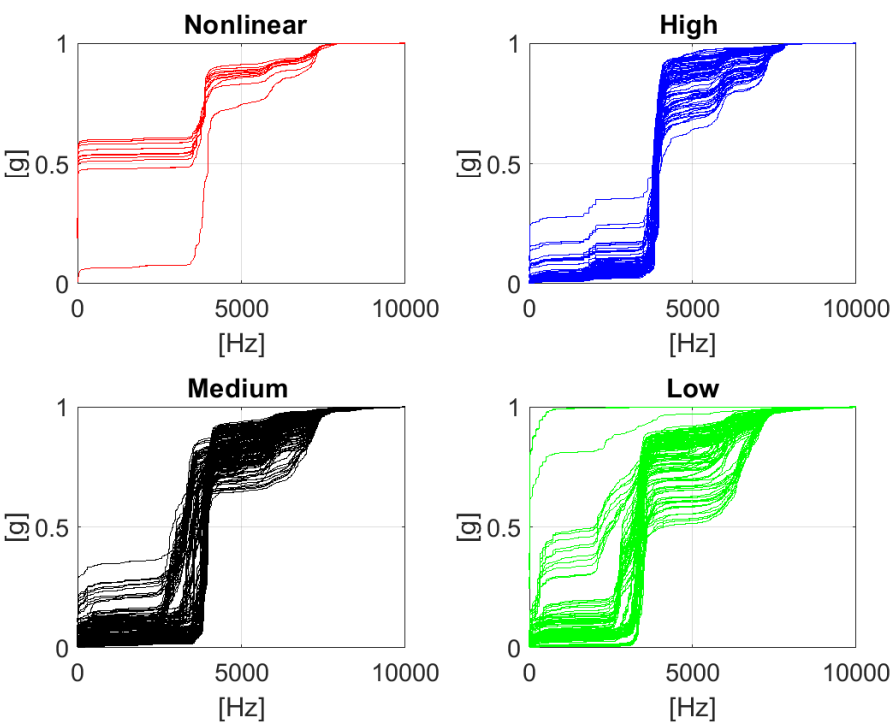

Figure A.3: Detail normalised PSD cumulative 


\section{A.3 Qualitative variations}

The train axle box environment is nonstationary due to the variation in the contact forces between the wheel and the rail track. This means that various contact irregularities lead to a wide range of effects on the vibration response which, unlike the wind turbine generator case, cannot be attributed only to the magnitude of the forces. The characterisation of the different environment-induced states is demonstrated for bearing $A$ at $5 \mathrm{~m} / \mathrm{s}$, as shown in Figure A.4. Four different states are identified:

- stable: a constant baseline vibration with the lowest rms and peak amplitude

- impulsive: short time disturbances with high peak-to-peak amplitude

- random: noisy signals of which the duration and amplitude exceed those of impulsive events

- random+impulsive: the combination of impulsive and random excitation.

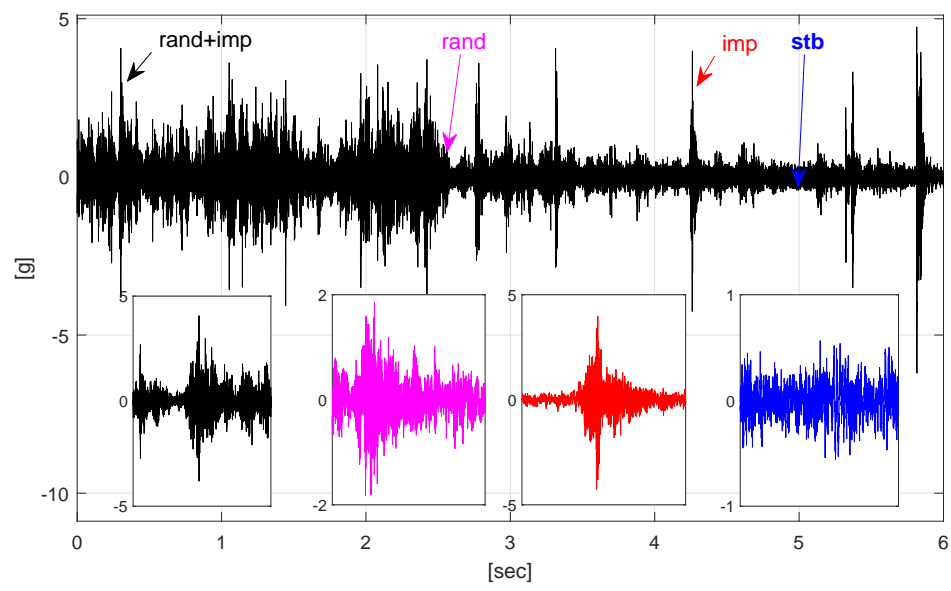

Figure A.4: Time waveform for bearing $A$ at $5 \mathrm{~m} / \mathrm{s}$ with distinct states:stable (blue), impact (red), random (magenta) and the random+impulsive (black).

The individual segments are analysed to identify the most distinctive features that can be used as a basis for classification. A comparison of the time waveform and the FFT for the stable and random states is presented in Figure A.5, and for the impact and random+impact states in Figure A.6. The effects of the impulsive excitations are more visible in the time waveforms than in the spectra. Conversely, the presence of random excitation is seen at frequencies above $4000 \mathrm{~Hz}$. 

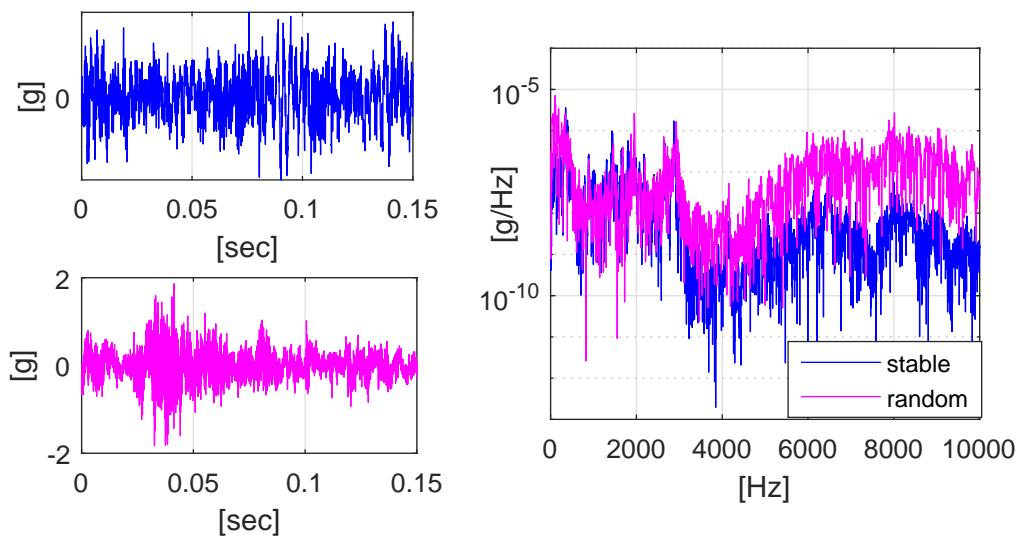

Figure A.5: Comparison of frequency distribution between stable and random states.
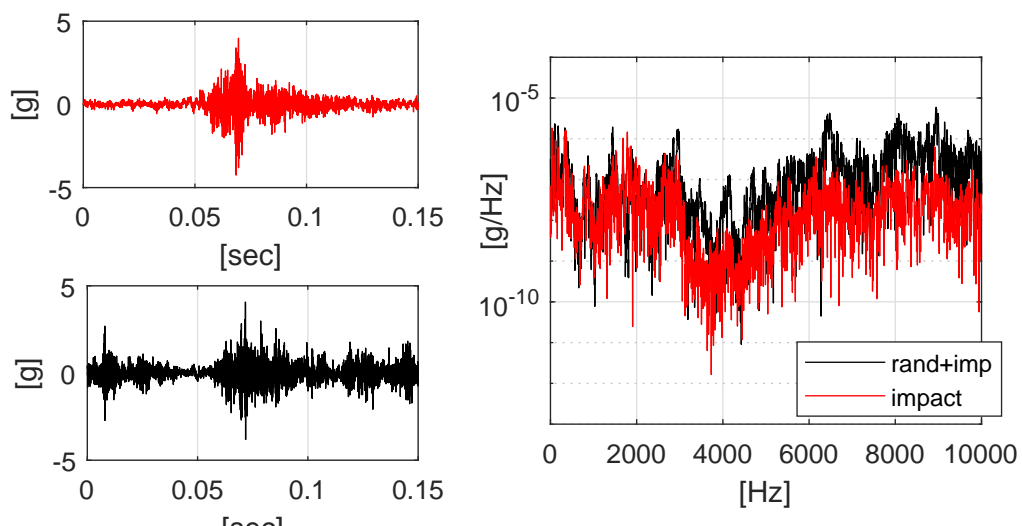

Figure A.6: Comparison of frequency distribution between impact and and random+impact states.

Based on the changes in energy distribution and impact behaviour a classification for the entire sample is performed. The first criterion is based on the energy distribution throughout the frequency spectrum (Equation A.1a)., where $X$ corresponds to the rms of the entire frequency range and $X_{0-F o}$ is the rms calculated in the frequency range between $0 \mathrm{~Hz}$ and Fo. The frequency limit $F q$ is defined based on the observations of the variability of the frequency range in presence of random environmental excitations as seen in Figures A.5 and A.6 The second criterion uses the peak-to-peak value of the sample analysed (Equation A.1b). 


$$
\begin{gathered}
c r 1=1-\frac{X_{0-F q}}{X} \\
c r 2=\operatorname{peak}(X)
\end{gathered}
$$

The two criteria are used to classify the different states of the signals according to the relations provided below.

- stable: $\mathrm{cr} 1 \leq$ ratio \& cr2 $<$ Impact $_{1}$

- impact: $\mathrm{cr} 1 \leq$ ratio \& cr2 $\geq$ Impact $_{1}$

- random: cr1 > ratio \& cr2 < Impact $_{2}$

- rand+imp: $\mathrm{cr} 1>$ ratio \& cr2 $\geq$ Impact $_{2}$

Note that there are two different limits for the evaluation of the second criterion, given the effect of the random excitation. The parameters for the classification are evaluated in accordance with the distribution of PSD cumulative. Specifically, the feature $f_{50}$ is used to validate the clusters. The final parameters are presented in Table A.2.

Table A.2: Classification parameter for railway bearing signals

\begin{tabular}{lcccc}
\hline & $F q[\mathbf{H z}]$ & ratio & Impact $_{1}$ & Impact $_{2}$ \\
\hline A 5 m/s & 3800 & 0.3 & 2 & 4 \\
A 13 m/s & 3500 & 0.15 & 5 & 7 \\
C 5 m/s & 1800 & 0.5 & 7 & 8 \\
C 13 m/s & 1100 & 0.5 & 20 & 30 \\
\hline
\end{tabular}

The classification algorithm is applied to the bearings $\mathrm{A}$ and $\mathrm{C}$ as seen in Figures A.7a and A.7b respectively. The upper frames in both figures show the classified time signal $(a)$ and the cumulative frequency distribution $(b)$. The lower frames correspond to scatter graphs that relate the signal dispersion $f_{50}$ with the overall $\mathrm{rms}$ (c) and peak-to-peak values (d). 
The derivative distribution show a balance increment of the signal power over the entire frequency range. This contrasts with the distributions observed for the lab set-up and the train cases, which showed a more steep increases. This shows that there is a greater number of resonances affected by the rail-wheel contact forces as well as by the damage.

In Figure A.7a the stable condition (blue) serves as a reference for the bearing behaviour in the absence of environmental disturbance or damage. In this case the presence of impacts (red) does not disturb the stability of the bearing since the frequency remains at the lower end. On the other hand, random excitation (magenta) and the superposition of random+impact (black) affect the stability of the system significantly once the frequencies scatter at higher frequency values.

The damage case shown in Figure A.7b reveals that the frequency range associated with bearing damage (i.e. $7000 \mathrm{~Hz}$ to $8000 \mathrm{~Hz}$ ) has a dominant character. For the stable and the impact events, the $f_{50}$ feature shows a more disperse behaviour than the random and random+impact states. Furthermore, the presence of random and random+impulse disturbances amplify the signal strongly, which is likely to accelerate the damage further. 


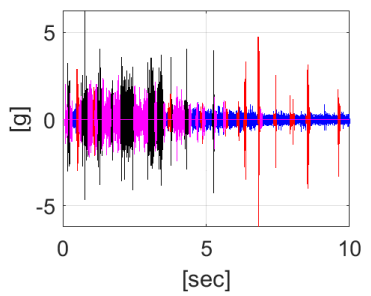

(a)

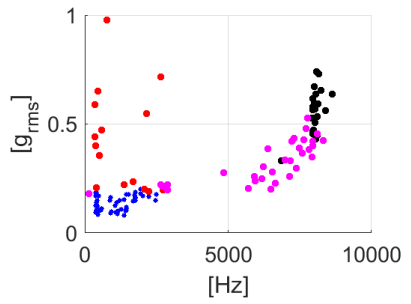

(c)

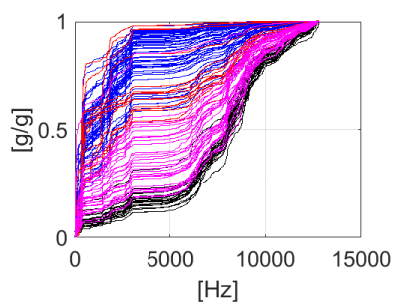

(b)

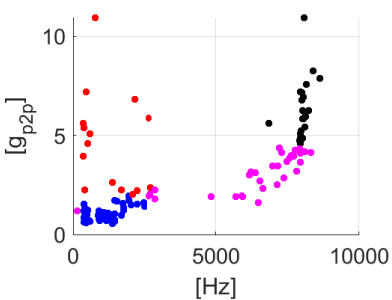

(d)

(a) Bearing $A$ at $5 \mathrm{~m} / \mathrm{s}$

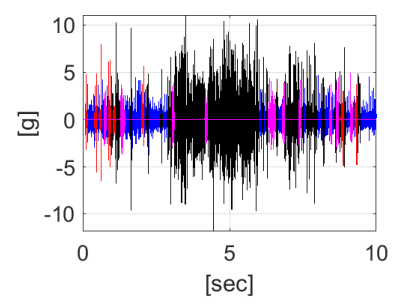

(a)

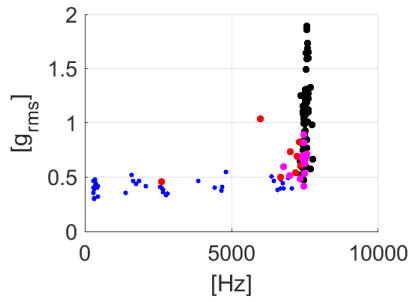

(c)

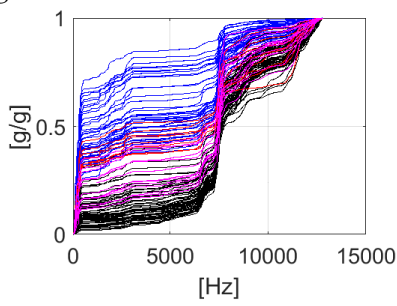

(b)

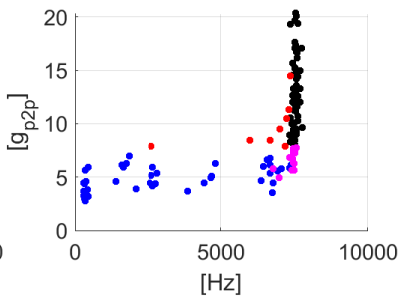

(d)

(b) Bearing C at $5 \mathrm{~m} / \mathrm{s}$

Figure A.7: Classification of instantaneous states: Time signal classified (a), Cumulative spectral distribution (b), rms values for $f_{50}$ feature (c), peak-to-peak values for $f_{50}$ feature $(d)$ 


\section{A.3.1 EMD analysis}

The stable category is used to analyse the effect of damage To this end, the stable condition of bearing $\mathrm{A}$ is compared with an equivalent sample from bearing $\mathrm{C}$, which is suspected of damage (Figure A.8)

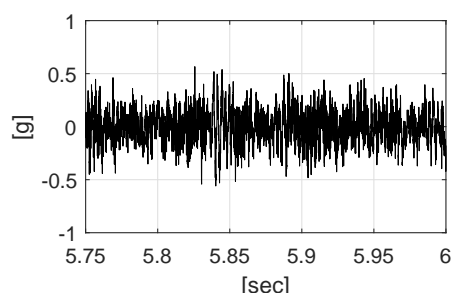

(a)

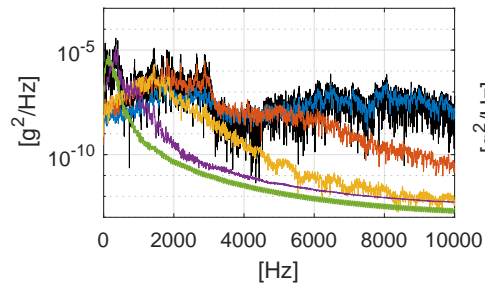

(c)

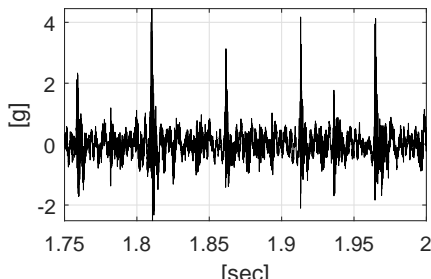

(b)

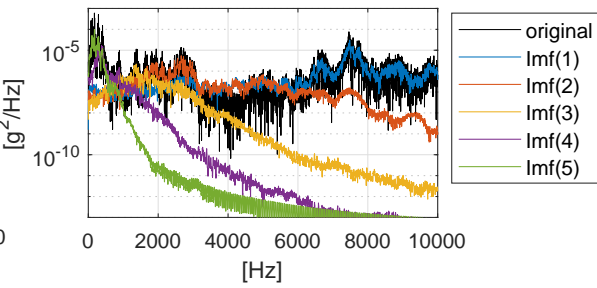

(d)

Figure A.8: Response analysis for stable state: time sample for bearings $A(a)$ and $C(b)$ and frequency distribution of IMFs for bearings $A(c)$ and $C(d)$

The time waveform for bearing A does not display any significant periodicity. On the other hand, bearing $C$ shows well-defined and regular peaks, which are likely to correspond to bearing damage. The frequency composition (Figures A.8 $c$ and d) shows that the damage forces substantially affect the higher frequency range as well as the low frequency range. This is confirmed by analysing the internal mode functions IMFs extracted using the empirical mode decomposition EMF.

The amplitude of each IMF for the different states is presented in Figures A.9 and A.10. Each scatter point represents the frequency at which the IMF peaks in relation to the rms value. It is seen that for bearing A the first IMF occurs between 5 $\mathrm{kHz}$ and $10 \mathrm{kHz}$. This confirms that the local bearing resonances are excited by the normal operational environment, even in the absence of damage. However, these resonances are more sensitive to random type of excitation than the impulsive type.

Furthermore, the lower IMFs reveal high activity below $4 \mathrm{kHz}$, and especially below $1000 \mathrm{~Hz}$. This confirms the consistent excitation of lower modes of the system, which are likely to correspond to the suspension and wheel modes. Further 
investigation of the wheel-rail excitation is necessary to verify the origins of these resonances.

For bearing $\mathrm{C}$ the effect of damage is focuses at $8 \mathrm{kHz}$. The fact that the first IMF displays the higher vibration content confirms that damage in the railway environment reflects more strongly at the local resonances of the bearing assembly. However, the vibration content does not decrease steadily at higher IMFs lower frequencies). This is most evident in the increment of the IMF 5 at the lower frequency ranges for the random and random+impact excitation (Figure A.10b c) and $d$ )). This suggests an important adverse effect in the suspension of the train bogie, with negative consequences for the passengers comfort and safety. 

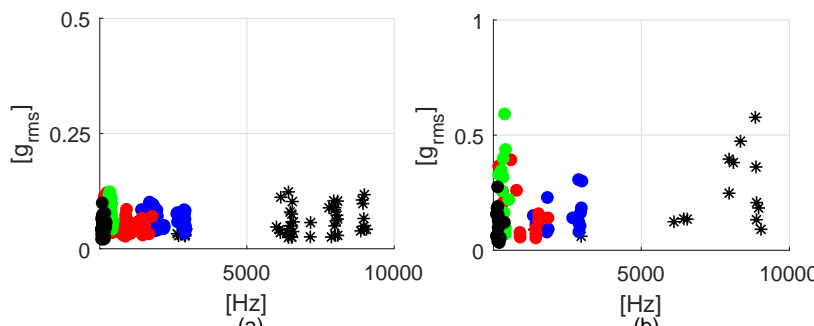

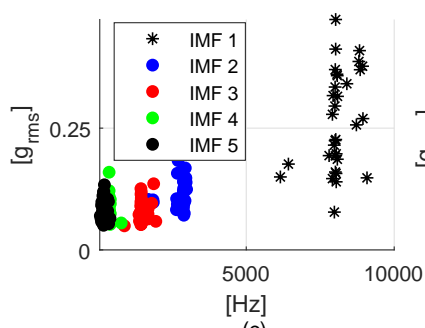

(c)

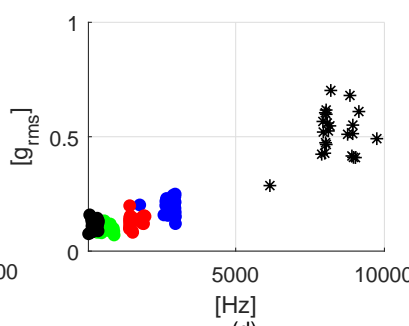

(d)

(a) Bearing A 5mps

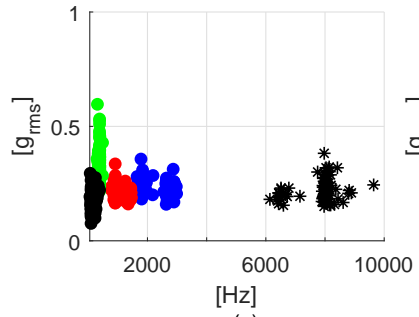

(a)

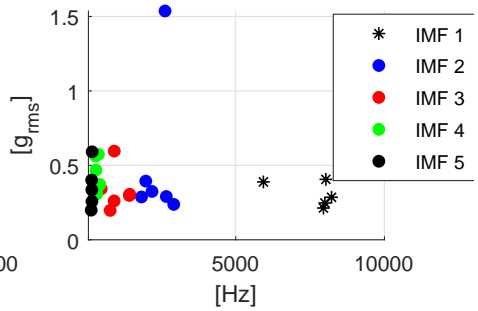

(b)

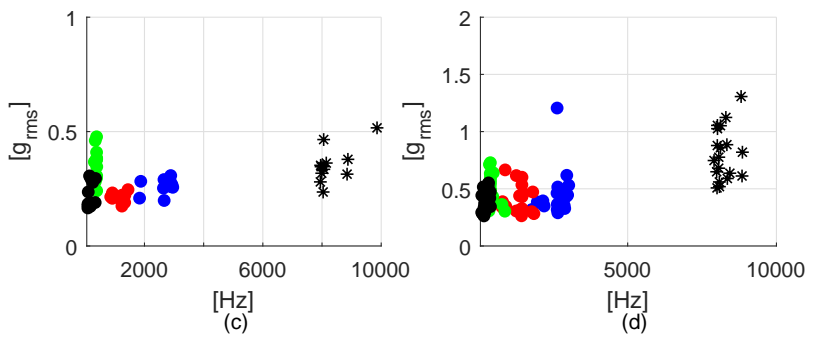

(b) Bearing A 13mps

Figure A.9: Scatter graphs of maximum frequency for IMFs versus rms for bearing A: stable (a), impact (b), random (c) and random+impact (d) 


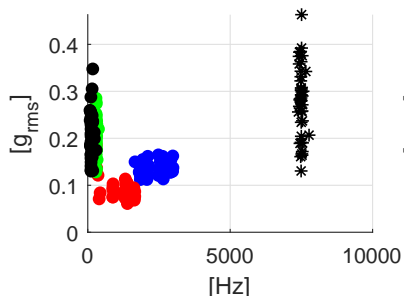

(a)

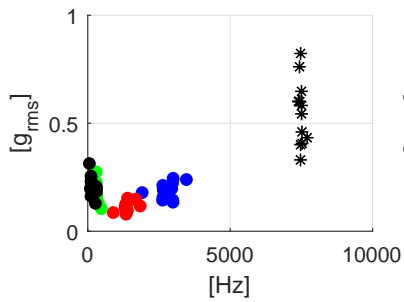

(c)

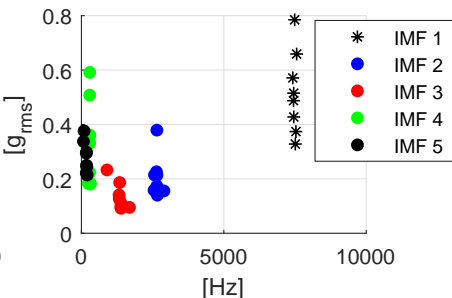

(b)

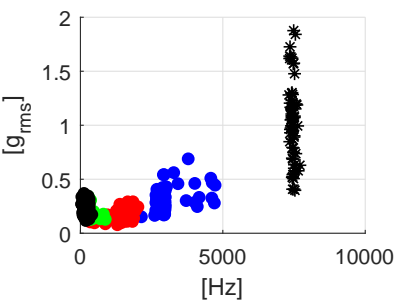

(d)

(a) Bearing C $5 \mathrm{mps}$

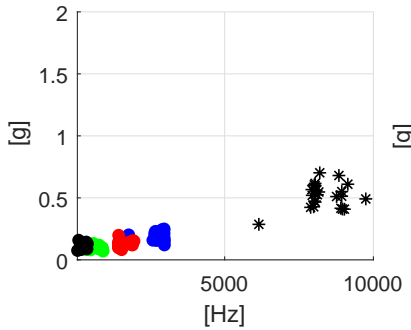

(a)

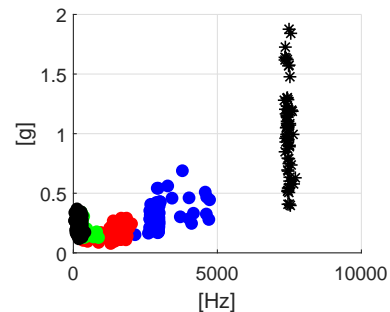

(c)

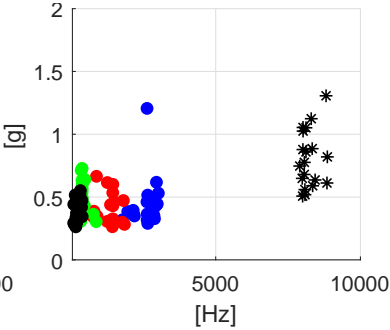

(b)

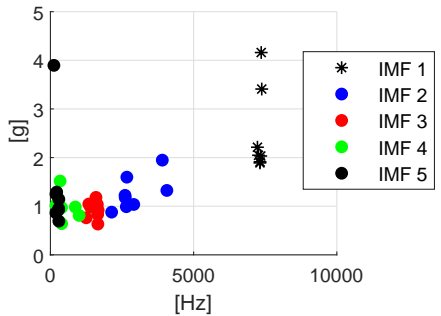

(d)

(b) Bearing C 13mps

Figure A.10: Scatter graphs of maximum frequency for IMFs versus rms for bearing C: stable (a), impact (b), random (c) and random+impact (d) 


\section{Acknowledgements}

The nonlinear path is never the most straightforward one, yet it is undoubtedly the one that offers the most enriching experiences.

The process that brought me to this thesis was a very interesting one. I befriended complexity and uncertainty, but also optimism. Now, after so long I can really turn the page. I want to thank all the people that made this book possible. You were there beside me, supporting me. I cannot give you back as much as you have given me. There won't even a party after the defence. So I need to express my gratitude to you here.

First at all, Leo. You helped me to rewrite the story of my Ph.D. Thank you very much for your interest and your patience. I appreciate each one of your motivating words. I needed them.

To Richard, I have learned from you the value of reliability. And not in the maintenance context. Thank you very much for helping me to write the thesis that I could be proud of.

To André, thanks for opening the door of the UT once more for me.

To Tiedo, thanks a lot for introducing the world of failure mechanisms to my vibration monitoring world.

To Debbie, you are not only the heart but also the soul of the department. People like you are a true gem: always giving more. Take good care of yourself.

To Inge and Annemarie, thank you ladies for being there always, making sure that all the little details were properly arranged. 
To Katherine, thank you for adopting me at Roskilde. That gave me the motivation for the last lap. Also, thank you for making room in your incredibly busy agenda.

To Eric, thank you for giving me a real job so I did not lose track of reality!

To the masters students Stefan, Joris, Chefiek, Raja and Felipe. Thanks for keeping up with me. I learned a lot from you.

To my friends, specially Amina, Susi, Coen, Sandra-Carlinas, Marteen B, Juan Carlos, Edgar, Inca, Laura-DK... I am glad you were there so I did not loose my sanity over all these years.

To my friends from the ICF, thank you for the shared experience of spirituality.

To my colleagues, I wish I could had enjoyed more of your company. Easy talks were always my favourite! To Jurnan and Laura: you guys have a great future ahead. Keep using your talents and charm to motivate young students and researchers.

Voor Opa, Oma en Sytske, bedankt dat jullie als een tweede familie voor me zijn. Jullie steun en gezelligheid betekenen veel voor mij.

Para mis padres, Gloria y Gonzalo, no hay un amor mas grande que el que ustedes me dan. Los extraño todos los dias pero conservo la esperanza que volveremos a estar cerca.

To Pequis and Pipe. You guys are definitely the smartest of the family. I know Nico couldn't be in better hands.

To Frans and Emmanuel, you guys brought an anchor into my life. Before you, my ship was drifting in the ocean. Now I can rest, I have a safe haven called home.

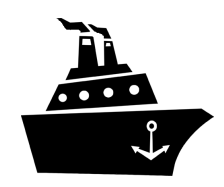




\section{About the author}

Andrea Sánchez Ramírez was born in Bogot, Colombia in 1982. She holds a bachelors degree in Mechanical Engineering from the Universidad Nacional de Colombia. In 2007 she had the good fortune of being awarded a scholarship in structural dynamics at the University of Twente, The Netherlands, completing the study cum laude.

In her working career she has occupied a variety of positions related to predictive maintenance, including field engineer and sales representative. Since 2010, Andrea has been working as an auditor for wind turbines using vibration analysis. In 2012 she joined the project WIBRATE, which focuses on the use of decentralised wireless sensor networks in vibration monitoring systems. In 2020 she finalised her PhD in Maintenance Engineering at the University of Twente. Andrea also holds a masters degree in Business Administration. She lives happily in Copenhagen, together with her partner and child. 
UNIVERSITY OF TWENTE. 


\section{Propositions \\ Accompanying the dissertation \\ ON THE COMPLEXITY OF FAILURE \\ A FUNCTIONAL APPROACH TO VIBRATION ANALYSIS}

1. The study of failure in complex systems requires an approach that acknowledges designed complexity and emerging complexity. (Chapter 1 of this thesis).

2. A comprehensive approach to failure assessment must integrate the structural aspects of component damage with the behavioural aspects of the functional relations between components. (Chapter 1 of this thesis).

3. Failure alters the designed balance that defines a system's performance and reliability. (Chapter 2 of this thesis).

4. Failure increases the entropy, uncertainty and interdependencies within a mechanical system. (Chapter 3 of this thesis).

5. Lateral vibration response is a direct indicator of the destabilization forces responsible for load distribution changes associated with complex failure, as in the case of imbalance and bearing damage (Chapter 4 of this thesis).

6. Maintainers of rotating mechanical systems often forget the designed functional complexity of the systems being maintained.

7. The data requirements needed for data-intensive approaches to failure modelling often conflict with the wishes of the machine owners, as nobody wants to let their systems run to failure.

8. The term complexity is often avoided due to its negative connotation, but in fact it should be credited more often for the emergent behaviours that bring order to the existing chaos.

9. The path to sustainability becomes easier if we, as mankind, learn the value of 'maintain and reuse' while avoiding 'produce to consume'.

10. Difficult questions are not only difficult to answer. They are also difficult to ask. 